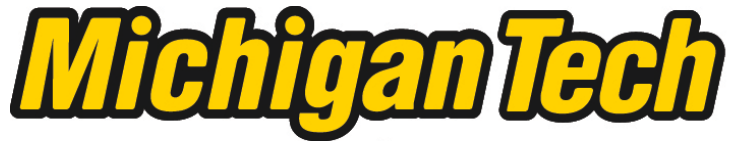 \\ Michigan Technological University Create the Future Digital Commons @ Michigan Tech
}

Dissertations, Master's Theses and Master's Reports - Open

Dissertations, Master's Theses and Master's

Reports

2002

Parameter estimation for transformer modeling

Sung Don Cho

Michigan Technological University

Follow this and additional works at: https://digitalcommons.mtu.edu/etds

Part of the Electrical and Computer Engineering Commons

Copyright 2002 Sung Don Cho

\section{Recommended Citation}

Cho, Sung Don, "Parameter estimation for transformer modeling", Dissertation, Michigan Technological University, 2002.

https://doi.org/10.37099/mtu.dc.etds/60

Follow this and additional works at: https://digitalcommons.mtu.edu/etds

Part of the Electrical and Computer Engineering Commons 


\title{
PARAMETER ESTIMATION FOR TRANSFORMER MODELING
}

\author{
By \\ SUNG DON CHO
}

\section{A DISSERTATION}

Submitted in partial fulfillment of the requirements

for the degree of

\section{DOCTOR OF PHILOSOPHY ELECTRICAL ENGINEERING}

MICHIGAN TECHNOLOGICAL UNIVERSITY

December 2002

Copyright (C) Sung D. Cho 2002 
This dissertation, "Parameter Estimation for Transformer Modeling", is hereby approved in partial fulfillment of the requirements for the degree of DOCTOR OF PHILOSOPHY in the field of Electrical Engineering.

\section{DEPARTMENT OF ELECTRICAL AND COMPUTER ENGINEERING}

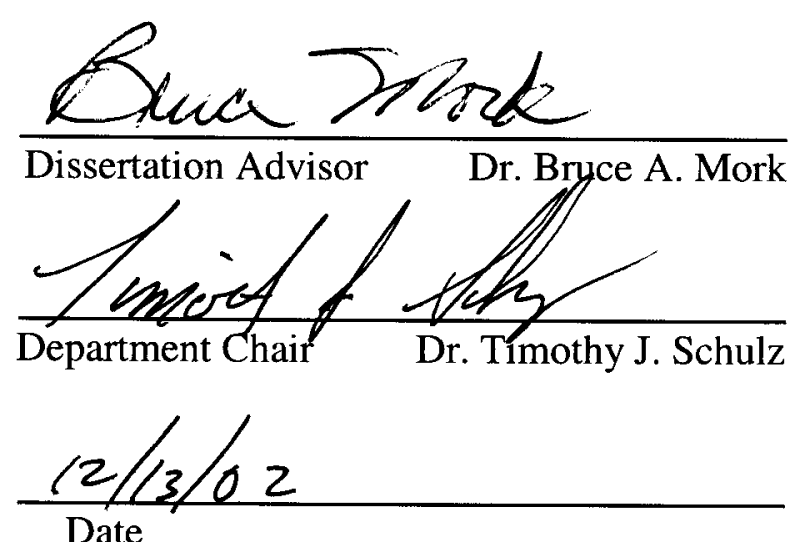




\begin{abstract}
Large Power transformers, an aging and vulnerable part of our energy infrastructure, are at choke points in the grid and are key to reliability and security. Damage or destruction due to vandalism, misoperation, or other unexpected events is of great concern, given replacement costs upward of $\$ 2 \mathrm{M}$ and lead time of 12 months. Transient overvoltages can cause great damage and there is much interest in improving computer simulation models to correctly predict and avoid the consequences.
\end{abstract}

EMTP (the Electromagnetic Transients Program) has been developed for computer simulation of power system transients. Component models for most equipment have been developed and benchmarked. Power transformers would appear to be simple. However, due to their nonlinear and frequency-dependent behaviors, they can be one of the most complex system components to model. It is imperative that the applied models be appropriate for the range of frequencies and excitation levels that the system experiences. Thus, transformer modeling is not a mature field and newer improved models must be made available.

In this work, improved topologically-correct duality-based models are developed for three-phase autotransformers having five-legged, three-legged, and shell-form cores. The main problem in the implementation of detailed models is the lack of complete and reliable data, as no international standard suggests how to measure and calculate parameters. Therefore, parameter estimation methods are developed here to determine the parameters of a 
given model in cases where available information is incomplete. The transformer nameplate data is required and relative physical dimensions of the core are estimated. The models include a separate representation of each segment of the core, including hysteresis of the core, $\lambda-i$ saturation characteristic, capacitive effects, and frequency dependency of winding resistance and core loss.

Steady-state excitation, and de-energization and re-energization transients are simulated and compared with an earlier-developed BCTRAN-based model. Black start energization cases are also simulated as a means of model evaluation and compared with actual event records. The simulated results using the model developed here are reasonable and more correct than those of the BCTRAN-based model. Simulation accuracy is dependent on the accuracy of the equipment model and its parameters. This work is significant in that it advances existing parameter estimation methods in cases where the available data and measurements are incomplete. The accuracy of EMTP simulation for power systems including three-phase autotransformers is thus enhanced.

Theoretical results obtained from this work provide a sound foundation for development of transformer parameter estimation methods using engineering optimization. In addition, it should be possible to refine which information and measurement data are necessary for complete duality-based transformer models. To further refine and develop the models and transformer parameter estimation methods developed here, iterative full-scale laboratory tests using high-voltage and high-power three-phase transformer would be helpful. 


\section{ACKNOWLEDGMENTS}

First, I would like to thank my wife, Miseon, and my son, Joonhyong, for helping me through the last three years. They've put their lives on hold for a few years so I could follow my dream.

I'd like to extend sincere gratitude to my advisor, Dr. Bruce Mork, for defining this project, giving me quite a few insights on it, and continually encouraging me. His ideas and insights were very precious to me.

I would like to thank my committee: Dr. Leonard Bohmann and Dr. Dennis Wittanen of the Electrical and Computer Engineering Department and Dr. Kee Moon of the Mechanical Engineering Department.

I am especially grateful to Kalyan Mustaphi and Xcel Energy for the financial support. 


\section{TABLE OF CONTENTS}

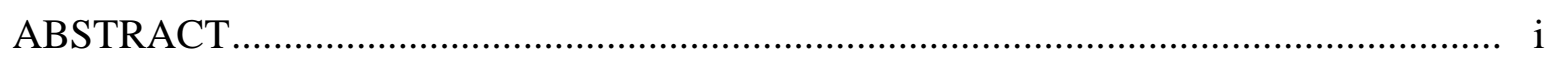

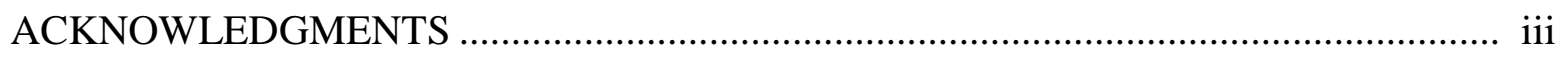

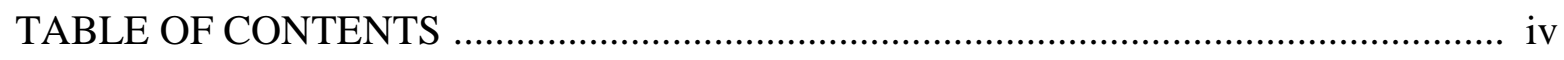

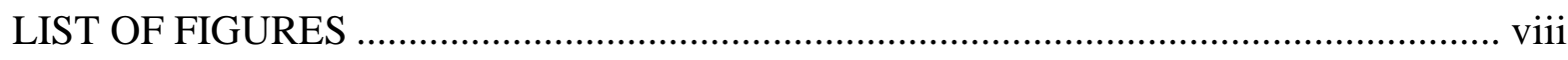

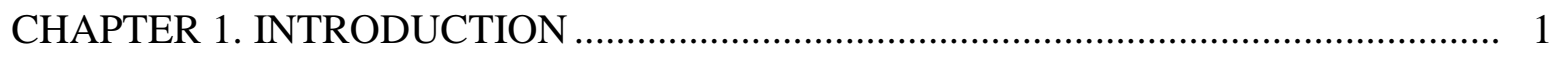

CHAPTER 2. INTRODUCTION TO TRANSFORMER MODELS ................................ 4

$2.1 \quad$ Basic Transformer Structure ................................................................. 4

2.2 STC (Saturable Transformer Component) Model ................................................ 6

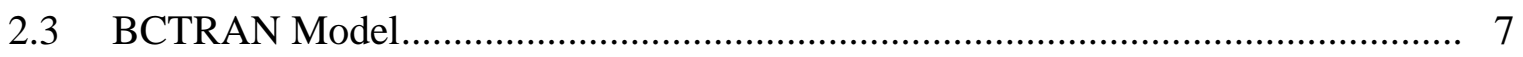

2.4 Duality Transformation …………………….............................................. 9

2.5 Coil/Winding Capacitance with Damping Resistance............................................. 12

2.6 Parameter Estimation using Engineering Optimization ......................................... 13

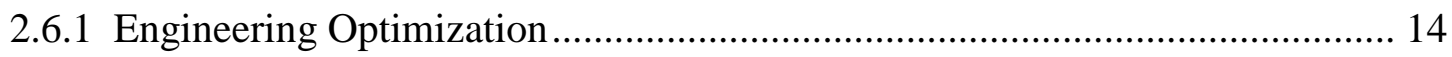




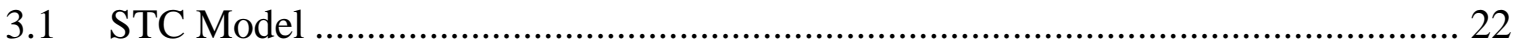

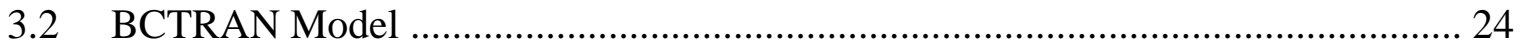

3.3 Duality-Based Model …………………………........................................ 27

CHAPTER 4. PARAMETERS FOR TRANSFORMER MODELS ................................... 30

4.1 Frequency-Dependency of Coil Resistance.......................................................... 31

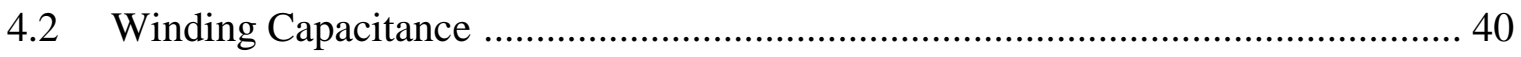

4.3 Magnetic Core Saturation ................................................................................ 49

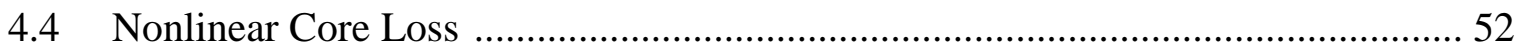

4.5 Separation of Core Loss ………………………........................................... 55

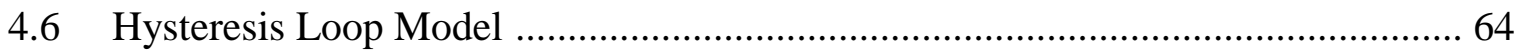

CHAPTER 5. DUALITY MODELS FOR THREE-PHASE TRANSFORMERS............... 73

5.1 Five-Legged Core Transformer ............................................................................ 73

5.2 Three-Legged Core Transformer......................................................................... 76

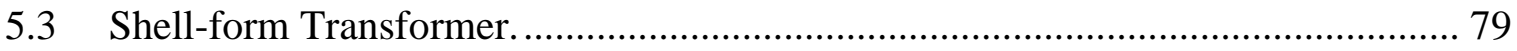


6.1 Five-Legged Core Transformer ............................................................. 82

6.1.1 Leakage Inductance .................................................................... 82

6.1.2 Practical Implementation of Leakage inductance ................................... 88

6.1.3 Core Saturation Model ........................................................................... 92

6.1.4 Core Loss Model................................................................................... 99

6.1.5 ATP Implementation of Overall Transformer Model .................................... 104

6.2 Three-Legged Core Transformer................................................................... 110

6.2.1 Leakage Inductance ....................................................................... 110

6.2.2 Core Saturation Model ........................................................................ 110

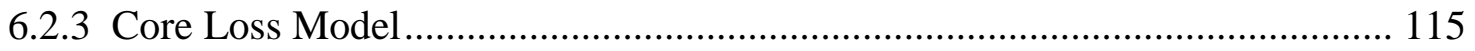

6.2.4 ATP Implementation of Overall Transformer Model .................................. 119

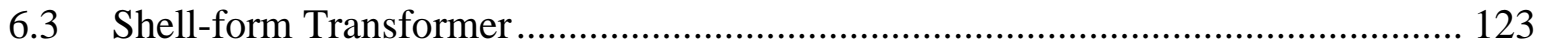

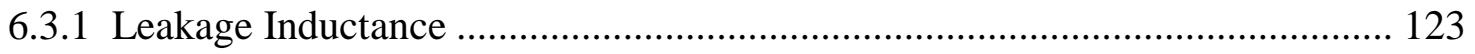

6.3.2 Core Saturation Model ............................................................................ 127

6.3.3 Core Loss Model...................................................................................... 131

6.3.4 ATP Implementation of Overall Transformer Model ................................... 135 
7.1 Comparison with BCTRAN Model.

7.2 Black Start Energization Cases at Inver Hills Substation.

7.2.1 System Description

7.2.2 Transformer Model 154

7.2.3 Transmission Line Model 155

7.2.4 Synchronous Generator Model 156

7.2.5 Case Study Results. 157

CHAPTER 8. CONCLUSION AND RECOMMENDATIONS FOR FUTURE WORK.... 165

REFERENCES 169

APPENDIX A: SAMPLE ATP DATA FILE 174

APPENDIX B: MATLAB CODE LISTING. 189

APPENDIX C: TRANSFORMER FACTORY TEST REPORT 209 


\section{LIST OF FIGURES}

Figure 2.1 Core Structure of Single-phase Transformer (Shell-form) ......................... 5

Figure 2.2 Core Structures of Three-phase transformers ........................................ 5

Figure 2.3 STC Model for Single-phase Two-winding Transformer .......................... 6

Figure 2.4 Terminal Representation for BCTRAN Model ....................................... 7

Figure 2.5 Equivalent Magnetic Circuit and Topological Development.................... 10

Figure 2.6 Equivalent Electrical Circuit Derived from Duality Transformation.......... 10

Figure 2.7 Equivalent Circuit for Capacitance with Damping Resistance .................. 13

Figure 3.1 STC Model for Three-phase Three-winding Autotransformer .................. 23

Figure 3.2 BCTRAN Model with External Core Elements for Three-phase

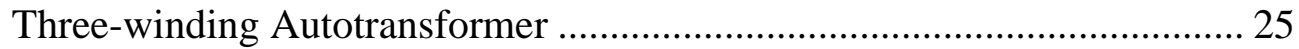

Figure 3.3 Three-phase Three-leg Core-type Transformer Structure ......................... 28

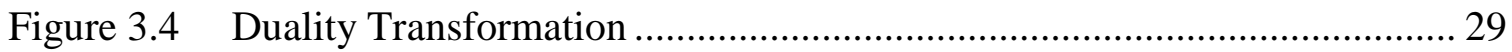

Figure 3.5 Equivalent Electric Circuit Derived from Duality Transformation............ 29

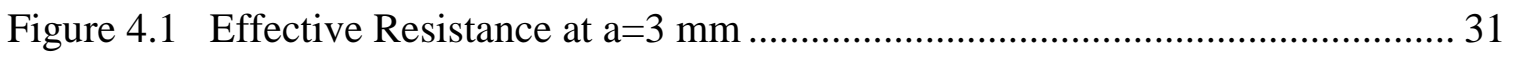

Figure 4.2 Typical L/R for Large Power Transformer ......................................... 32

Figure 4.3 Typical Slope of Effective Resistance for Large Power Transformer .......... 32

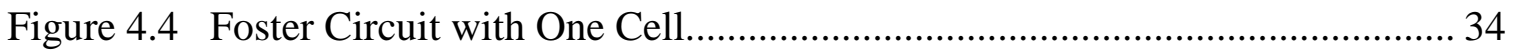


Figure 4.5 Effective R and L by Equation (4.3) with Foster Circuit with One Cell...... 34

Figure 4.6 Effective R and L by Least Square Curve Fitting with Foster Circuit

with One Cell

Figure 4.7 Series Foster Circuit with Two Cells....................................................... 36

Figure 4.8 Effective R and L by Foster Circuit with Two Cells................................ 36

Figure 4.9 Effective $\mathrm{R}_{\mathrm{HL}}$ and $\mathrm{L}_{\mathrm{HL}}$ for Example Transformer by Foster Circuit with Two Cells 39

Figure 4.10 Equivalent Circuit for Capacitance 40

Figure 4.11 Capacitances of Concentric Winding 44

Figure 4.12 Capacitances of Pancake Winding 44

Figure 4.13 Capacitances for Three-winding Three-phase Autotransformer. 45

Figure 4.14 Examples of Saturation Curve Fitting using Frolich Equation 50

Figure 4.15 Derived Saturation Curve 51

Figure 4.16 Derived Saturation Curve 52

Figure 4.17 Examples of 60-Hz Core Loss Curve using Frolich Equation 54

Figure 4.18 Examples of DC Hysteresis Loss Curve using Frolich Equation 54

Figure 4.19 Equivalent Circuit for Separated Core Loss Model 57

Figure 4.20 Function Values at Each Iteration by Successive LP method 59

Figure 4.21 Local Optima for Equation 4.22 (left) and Equation 4.23 (right) .59

Figure $4.22 \mathrm{v}-\mathrm{i}_{\mathrm{H}}$ and $\lambda-\mathrm{i}_{\mathrm{H}}$ Plot at $110 \% \mathrm{~V}$ and $60 \mathrm{~Hz}$ 62 
Figure 4.23 Time vs. v, $\lambda, \mathrm{i}_{\mathrm{H}}$ Waveforms at $110 \% \mathrm{~V}$ and $60 \mathrm{~Hz}$

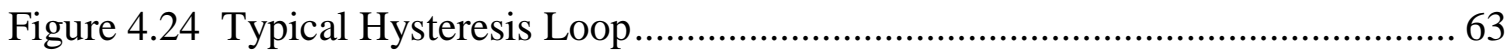

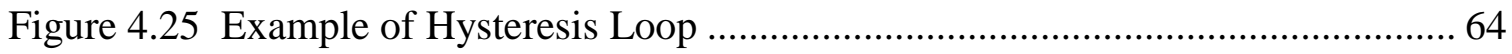

Figure 4.26 Examples of Hysteresis Loop Using Two Hyperbolic Functions ............... 65

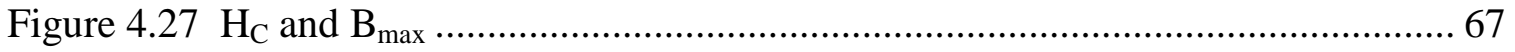

Figure 4.28 Left and Right Displacements of Resistive Hysteresis Current .................. 68

Figure 4.29 DC Hysteresis Loop Generated by the Model.......................................... 68

Figure 4.30 DC Hysteresis Loop Generated by the Model........................................ 69

Figure 4.31 Hysteresis Loop Generated by Decaying B with Time ............................. 69

Figure 4.32 Core Model for ATP Implementation ................................................ 70

Figure 4.33 Block Diagram for DC Hysteresis Loop using TACS ............................. 71

Figure 4.34 DC Hysteresis Loop Generated by the Model........................................ 72

Figure 4.35 Hysteresis Loop Generated by Deaying B with Time ............................... 72

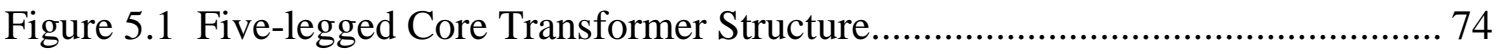

Figure 5.2 Magnetic Circuit for Five-Legged Core Transformer ............................... 74

Figure 5.3 Equivalent Electric Circuit for Five-Legged Core Transformer .................. 75

Figure 5.4 Three-legged Core Transformer Structure ............................................ 77

Figure 5.5 Magnetic Circuit for Three-legged Core Transformer ............................... 77

Figure 5.6 Equivalent Electric Circuit for Three-legged Core Transformer ................. 78

Figure 5.7 Shell-form Transformer Structure .................................................. 80 
Figure 5.8 Magnetic Circuit for Shell-form Transformer.

Figure 5.9 Equivalent Electric Circuit for Shell-form Transformer ............................ 81

Figure 6.1 Transformer Cross Section with Three Windings and MMF Distributions ... 83

Figure 6.2 Electrical Equivalent Circuits for Leakage Reactance................................ 85

Figure 6.3 Electrical Equivalent Circuits with Breakdown of Leakage Effects .............. 87

Figure 6.4 Three-winding Equivalent Circuit from Test Report ................................. 88

Figure 6.5 Cross-Section with Main Leakage Paths for Concentric Windings ............... 91

Figure 6.6 Dimension of Five-Leg Core-type Transformer...................................... 92

Figure 6.7 Magnetic Equivalent Circuit for Five-Legged Transformer ....................... 93

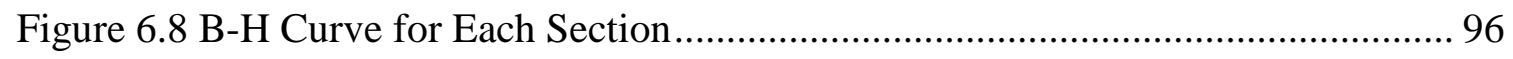

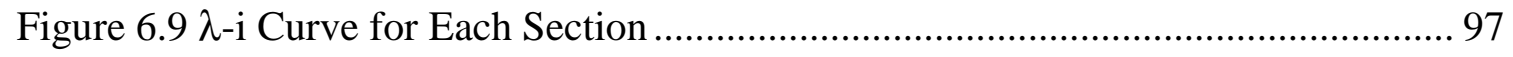

Figure 6.10 Current Waveforms for Each Section at 100\%v V ................................. 98

Figure 6.11 Current Waveforms for Each Line at 100\% Voltage ............................... 98

Figure 6.12 Core Loss Curve for Five-legged Core Transformer .............................. 100

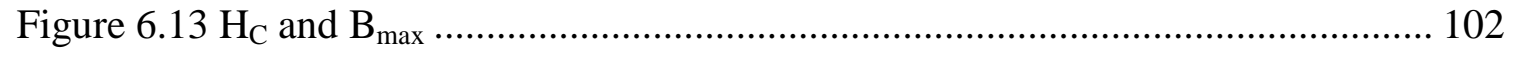

Figure 6.14 Left and Right Displacements of Resistive Hysteresis Current ................ 103

Figure 6.15 D C Hysteresis Loop Generated By the Model ..................................... 103

Figure 6.16 Frequency-Dependency Resistance Model R(f) Implementation in ATP.. 105

Figure 6.17 Equivalent Circuit for Five-Legged Core Transformer,Implemented in ATP106

Figure 6.18 DC Hysteresis Loop Generated By ATP ............................................ 108 
Figure 6.19 Eddy Current $\left(\mathrm{i}_{\mathrm{E}}\right)$ and Hysteresis Current $\left(\mathrm{i}_{\mathrm{H}}\right)$ Waveforms at 100\%V ...... 108

Figure 6.20 Mangnetizing Current Waveforms of Leg- 1, Yoke-AB and Outer Limb . 108

Figure 6.21 Current Waveforms for Each Line at 100\% Voltage .............................. 109

Figure 6.22 Current Waveforms for Each Winding at 100\% Voltage......................... 109

Figure 6.23 Dimension of Three-Legged Core Type Transformer.............................. 111

Figure 6.24 Magnetic Equivalent Circuit for Three-Legged Transformer ................... 111

Figure 6.25 B-H Curves for Each Section ...................................................... 113

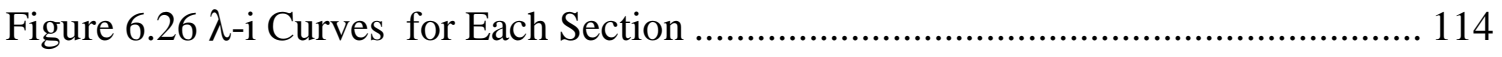

Figure 6.27 Current Waveforms for Each Section at 100\% Voltage.......................... 114

Figure 6.28 Current Waveforms for Each Line at 100\% Voltage .............................. 115

Figure 6.29 Core Loss Curve for Three-legged Core Transformer ............................. 116

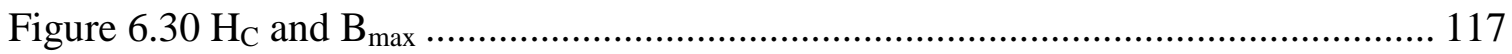

Figure 6.31 Left and Right Displacements of Resistive Hysteresis Current .................. 118

Figure 6.32 DC Hysteresis Loop Generated By the Model ...................................... 118

Figure 6.33 Equivalent Circuit for Three-legged Core Transformer,

Implemented in ATP................................................................. 120

Figure 6.34 DC Hysteresis Loop Generated By ATP............................................. 121

Figure 6.35 Eddy Current $\left(\mathrm{I}_{\mathrm{E}}\right)$ and Hysteresis Current $\left(\mathrm{I}_{\mathrm{H}}\right)$ Waveforms at 100\%V ...... 121

Figure 6.36 Mangnetizing Current Waveforms of Leg 1 and Yoke A-B at 100\%V ..... 121

Figure 6.37 Line Current Waveforms for Tertiary at 100\% Voltage ........................... 122 
Figure 6.38 Winding Current Waveforms for Tertiary at 100\% Voltage

Figure 6.39 Cross-Section with Main Leakage Paths for Pancake type Winding ....... 123

Figure 6.40 Cross-Section with Main Leakage Paths for Pancake type Winding ........ 124

Figure 6.41 Dimension of Shell-form Transformer 127

Figure 6.42 Magnetic Equivalent Circuit for Shell-form Transformer. 128

Figure 6.43 B-H and $\lambda$-i Curves for Each Section 130

Figure 6.44 $\lambda-i$ Magnetization Curves for Each Section 130

Figure 6.45 Current Waveforms for Each Line at 100\% Voltage 131

Figure 6.46 Core Loss Curve for Shell-form Transformer 133

Figure 6.47 Hc and Bmax 134

Figure 6.48 Left and Right Displacements of Resistive Hysteresis Current 134

Figure 6.49 DC Hysteresis Loop Generated by the Model 135

Figure 6.50 Equivalent Circuit for Shell-form Transformer, Implemented in ATP 136

Figure 6.51 DC Hysteresis Loop Generated by ATP 137

Figure 6.52 Eddy Current $\left(\mathrm{I}_{\mathrm{E}}\right)$ and Hysteresis Current $\left(\mathrm{I}_{\mathrm{H}}\right)$ Waveforms at 100\%V ..... 137

Figure 6.53 Mangnetizing Current Waveforms of Leg 2 and Mid Limb A-B (Leg-7) at $100 \% \mathrm{~V}$

Figure 6.54 Current Waveforms for Each Line at 100\% Voltage 138

Figure 6.55 Current Waveforms for Each Winding at 100\% Voltage 138

Figure 7.1 Transformer Magnetizing Current for Three Legs 142

Figure 7.2 Transformer Core Flux - Magnetizing Current Plot for Leg-1. 143 
Figure 7.3 Transformer Core Loss Currents for Three Legs

Figure 7.4 Transformer Core Flux - Core Loss Current Plot for Leg-1

Figure 7.5 Transformer Core Flux - No-Load Current Plot for Leg-1 146

Figure 7.6 Transformer No-Load Currents for 115-kV Lines 147

Figure 7.7 Transformer Core Fluxes at Legs after De-Energizing 148

Figure 7.8 Transformer Core Flux vs. Core Loss Current Plot after De-Energizing..... 149

Figure 7.9 Transformer Core Fluxes after Re-Energizing..... 150

Figure 7.10 Transformer 115-kV Line Currents after Re-Energizing

Figure 7.11 A Single-Line Diagram for Black Start Study

Figure 7.12 Block Diagram for Generator Excitation System. 156

Figure 7.13 115-kV CB 5P147 B-phase Voltage (Top) and Current (Bottom) just after 115-kV CB 5P147 Energization

Figure 7.14 115-kV CB 5P147 B-phase Voltage (Top) and Current (Bottom) 1.5 second after $115-\mathrm{kV}$ CB 5P147 Energization.

Figure 7.15 115-kV CB 5P147 B-phase Voltage (Top) and Current (Bottom) 3 seconds after 115-kV CB 5P147 Energization

Figure 7.16 115-kV CB 5P147 C-phase Voltage (Top) and Current (Bottom) Just after Energization of 345-kV Transformer No. 9 at BLL. 162

Figure 7.17 115-kV CB 5P147 C-phase Voltage (Top) and Current (Bottom) 1.5 second after Energization of 345-kV Transformer No. 9 at BLL 163

Figure 7.18 115-kV CB 5P147 C-phase Voltage (Top) and Current (Bottom) 3 Seconds after Energization of 345-kV Transformer No.9 at BLL 164 


\section{CHAPTER 1}

\section{INTRODUCTION}

ATP (Alternative Transient Program, the royalty-free version of the EMTP - the Electromagnetic Transients Program) was developed for computer simulation of power system transients. Component models for power system equipment have also been developed and benchmarked. Power transformers would appear to be simple. However, due to their nonlinear and frequency-dependent behaviors, they can be one of the most complex system components to model. It is imperative that the applied models be appropriate for the range of frequencies and excitation levels that the system experiences. Transformer modeling is not a mature field and newer improved models must be made available in ATP packages. Further, there is a lack of published guidance on recommended modeling approaches. And there is typically not enough detailed design or test information available to determine the parameters for a given model.

The purpose of this dissertation project is to develop improved transformer models and parameter estimation methods that can efficiently utilize the limited available information such as factory test reports, core type and core dimension.

Chapter 2 gives the results of a literature search, provides an overview of transformers, and presents some of the more commonly-used models presently being used in transient simulation. 
Chapter 3 describes and gives insights on the parameters and advanced equivalent circuit models that can be applied to three-phase transformers. The pros and cons of some existing models are briefly discussed and some examples presented. The main problem with these representations is the lack of reliable implementation data, as no international standard suggests how to measure and calculate the needed parameters.

Chapter 4 refines the existing approaches for parameters and characteristics used by the equivalent circuits presented in Chapter 3. To improve our understanding of the details of transformer modeling, the nonlinear and frequency-dependent characteristics are studied. Parameter estimation methods are developed to determine the parameters of a given model in cases where incomplete information is available. This parameter estimation problem inherently transforms to a constrained optimization problem in engineering, because the model parameters must be selected so that the model fits all the available data and measurements as closely as possible.

Duality-based transformer models are topologically correct and can be used to accurately represent each segment of the magnetic core. Chapter 5 develops the dualitybased equivalent circuit models for three-phase five-legged, three-phase three-legged, and three-phase shell-form autotransformers for ATP implementation. However, available information is typically not enough to determine the parameters for these duality-based transformer models.

Chapter 6 develops the parameter estimation methods for the duality-based models of Chapter 5. Physical dimension and the nonlinear and frequency-dependent characteristics are implemented in the parameter estimation. Mathematical description of 
parameters and their interrelationships are refined. The models include a separate representation of each segment of the core, including hysteresis of the core, $\lambda-i$ saturation characteristic, capacitive effects, and frequency-dependency of winding resistance and core loss.

Chapter 7 presents the results of ATP simulations used in benchmarking. Models developed in Chapter 6 are used to compare simulation results to actual event records. Steady-state excitation and de-energization and re-energization transients are simulated and compared with the results of an earlier BCTRAN-based model. The performance of the equivalent circuit and observations on parameters are summarized.

Chapter 8 contains the conclusions and summary of this work. Based on the results, some recommendations and suggestions for future research work are provided. These suggestions are intended to further improve the performance of the models and clearly set a starting point for researchers who wish to continue the work in this area. 


\section{CHAPTER 2}

\section{INTRODUCTION TO TRANSFORMER MODELS}

\subsection{Basic Transformer Structure}

A transformer consists of core, coil, tank, insulation and other accessories. The iron core is made of laminations to reduce eddy current losses and the material is silicon alloy to reduce hysteresis losses and to improve magnetization characteristics. Reducing the thickness of laminations reduces the eddy current losses in the core. There are two classes of coils - concentric (cylindrical) windings and interleaved (pancake) windings. For concentric windings, the high-voltage coil is typically wound over the low-voltage coil to

obtain good coupling between windings. For interleaved windings, the high-voltage and the low-voltage windings are stacked in alternating pancake-shaped coils. In actual design, many modifications are used by the various manufactures. Paper, pressboard, mineral oil, and epoxy resin are used for insulation [22].

Examples of windings and core structures for single-phase and three-phase transformers are shown in Figures 2.1 and 2.2. The quantitative expressions for a coilwound magnetic circuit are given in Equations (2.1) through (2.7) [7]. 


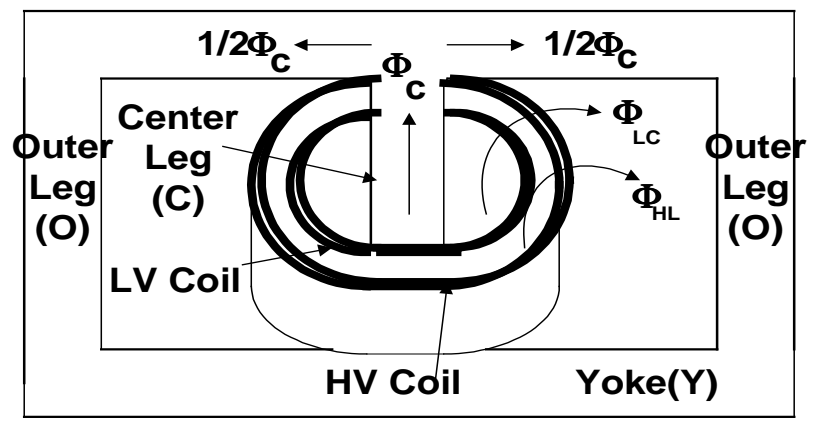

Figure 2.1 Core Structure of Single-phase Transformer (Shell-form)

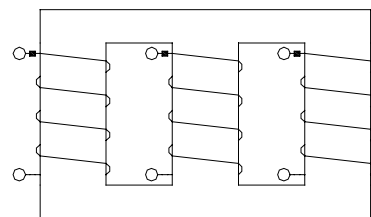

(a) Three-legged Core

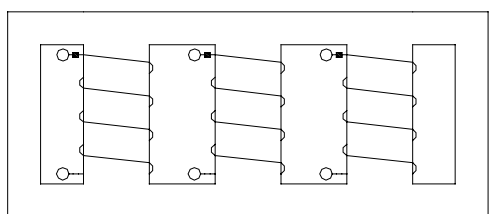

(b) Five-legged Core

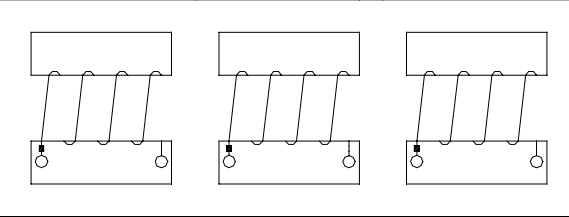

(c) Shell-form

Figure 2.2 Core Structures of Three-phase transformers

$$
\begin{array}{lll}
\Re=\frac{i}{\mu \cdot A} & (2.1) & B=\frac{\Phi}{A}=\mu \cdot H \\
M M F=N \cdot i & (2.2) & \lambda=N \cdot \Phi=L \cdot i \\
\Phi=\frac{M M F}{\Re} & (2.3) & L=\frac{N^{2}}{\Re} \\
H=\frac{M M F}{l} & (2.4) &
\end{array}
$$

Where, $\mathfrak{R}$ : Reluctance, $i$ : current, $\mu$ : permeability, A: area of core, $\Phi:$ flux, $M M F$ : magnetomotive force, $N$ : number of turns, $H$ : magnetic field intensity, $B:$ flux density, $l$ : length of core, $L$ : inductance, $\lambda:$ flux linkage 


\subsection{STC (Saturable Transformer Component) Model}

ATP is a digital simulation program for transient phenomena of an electromagnetic system. It has been continuously developed through international contributions. Interfacing capability to the program modules TACS (Transient Analysis of Control Systems) and MODELS (a simulation language) enables modelling of control systems and components with nonlinear characteristics.

ATP offers two different transformer models. These two components are referred to as STC and BCTRAN models. STC is a built-in model that can be implemented with and without saturable core representation. It is limited to single-phase or three-phase banks made up of single-phase units. No mutual coupling between the phases can be taken into account. In addition, it is not possible to represent the differences between the positive and the zero sequence paths. Therefore, unequal phase reluctances and the nonlinear interactions between limbs of the core cannot be taken into account $[6,17]$.

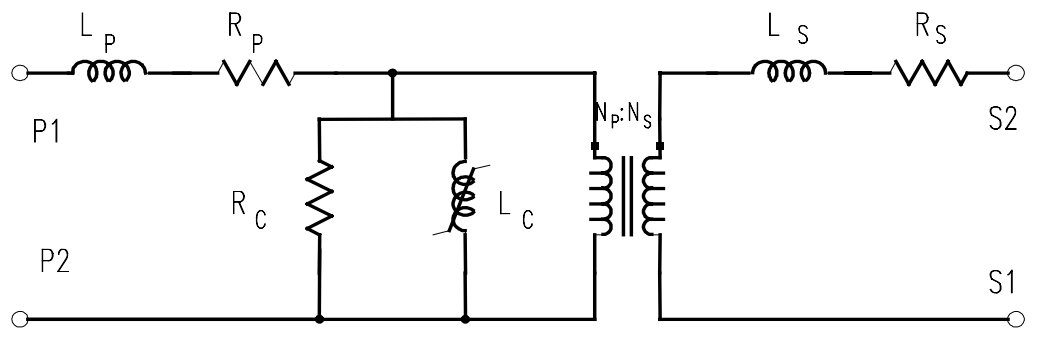

Figure 2.3 STC Model for Single-phase Two-winding Transformer [18]

Figure 2.3 gives STC model for single-phase transformer of Figure 2.1. This model has a built-in core representation $\left(\mathrm{R}_{\mathrm{C}}\right.$ and $\left.\mathrm{L}_{\mathrm{C}}\right)$, which is connected at the ideal coupling transformer. A piecewise linear $\lambda-i$ (flux linked vs. current) curve is defined point-bypoint, with a linear resistance connected in parallel. As an approximation, the 
manufacturer's RMS saturation curve of voltage vs. current may be input and converted to peak flux linkages and peak current using the supporting routine SATURATION [18].

Required input parameters are: leakage impedance, winding resistance and turns ratio. This model is simple to use, but is limited to single-phase or three-phase banks of single-phase units and may be numerically unstable because of negative inductance in the equivalent circuit of the three-winding transformer [8,39].

\subsection{BCTRAN Model}

BCTRAN is the supporting routine of the EMTP program which creates an impedance or admittance matrix representation of the transformer, without taking into account the saturable core effects, from transformer ratings and factory test data. From Brandwajn and Dommel [4], single-phase and three-phase N-winding transformers can be represented in the form of a branch impedance or admittance matrix, derived from short-circuit and open-circuit nameplate data.

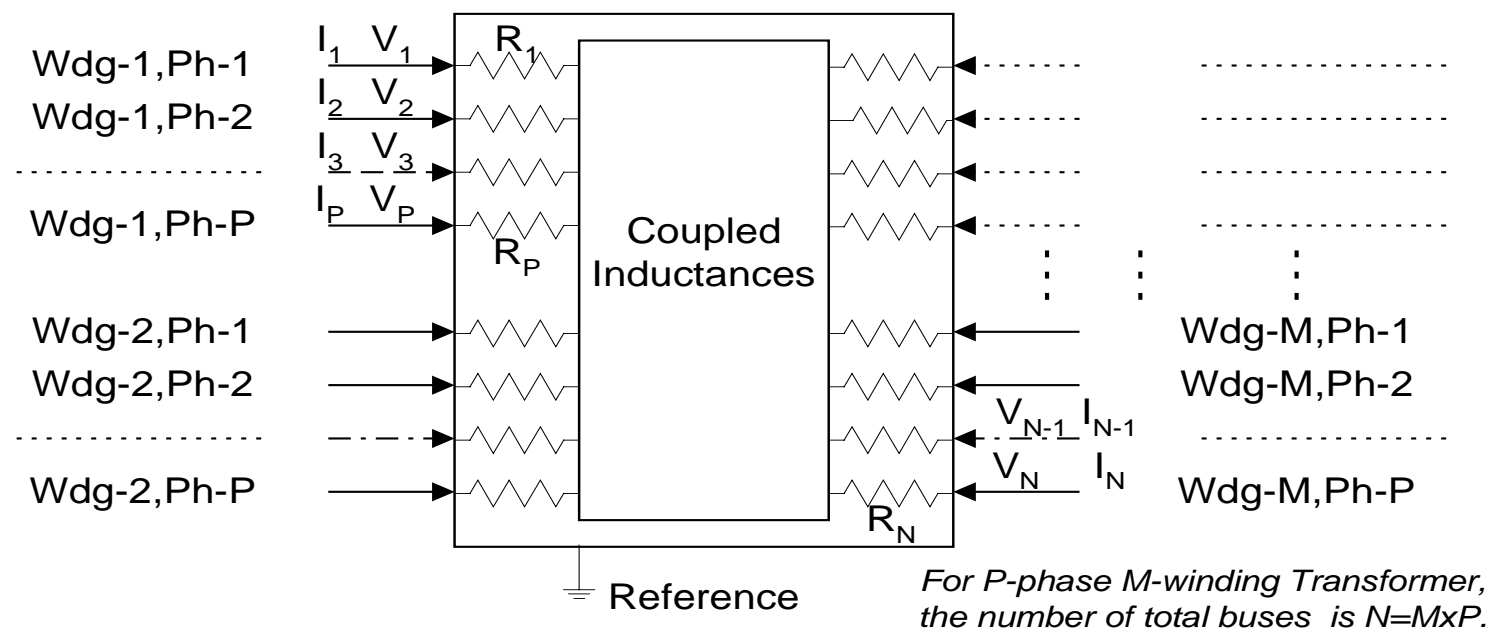

Figure 2.4 Terminal Representation for BCTRAN Model 
The BCTRAN routine can create an AR model of the leakage impedances of the transformer to avoid the problem of inverting a singular $[\mathrm{L}]$, where $[\mathrm{A}]$ is the inverse of $[\mathrm{L}]$, as in the Equation (2.9). Where $[\mathrm{L}]$ is the inductance matrix, $[\mathrm{R}]$ is the resistance matrix, $[\mathrm{v}]$ is a vector of terminal voltages, and [i] is the current vector. As in other threephase network components, the positive and zero sequence values from test data (excitation and short-circuit data) are used. Therefore, the representation of unbalance between phases is possible [17].

The elements of the $[\mathrm{L}]$ matrix are self inductances and mutual inductances. The copper-loss resistances form a $\mathrm{N} \times \mathrm{N}$ diagonal matrix $[\mathrm{R}]$, each element of which corresponds to its respective winding $[8,17]$.

$$
\begin{aligned}
{\left[\begin{array}{c}
v_{1} \\
v_{2} \\
\vdots \\
v_{N}
\end{array}\right]=} & {\left[\begin{array}{cccc}
R_{11} & 0 & \cdots & 0 \\
0 & R_{22} & \cdots & 0 \\
\vdots & \vdots & \ddots & \vdots \\
0 & 0 & \cdots & R_{N N}
\end{array}\right]\left[\begin{array}{c}
i_{1} \\
i_{2} \\
\vdots \\
i_{N}
\end{array}\right]+\left[\begin{array}{cccc}
L_{11} & L_{12} & \cdots & L_{1 N} \\
L_{12} & L_{22} & \cdots & L_{2 N} \\
\vdots & \vdots & \ddots & \vdots \\
L_{N 1} & L_{N 2} & \cdots & L_{N N}
\end{array}\right] \frac{d}{d t}\left[\begin{array}{c}
i_{1} \\
i_{2} \\
\vdots \\
i_{N}
\end{array}\right] } \\
& {[L]^{-1} \cdot[v]=[L]^{-1} \cdot[R] \cdot[i]+\frac{d}{d t} \cdot[i] }
\end{aligned}
$$

The iron-loss resistances are placed in parallel with each winding. Exciting current effects can be linearized and left in the matrix description, which can lead to the simulation errors when the core saturates. Alternately, excitation may be omitted from the matrix description and attached externally at the model's terminals in the form of nonlinear core elements. Such an externally attached core equivalent must have the same topology as the duality transformation for the complete transformer, however, so attaching this core equivalent to the external terminals is not topologically correct. 
In this model, it is possible to represent the differences between the positive and the zero sequence paths. However, unequal phase reluctances and nonlinear interactions between limbs of the core cannot be taken into account. As input data, manufacturer data including zero and positive sequence impedances from the binary short-circuit tests is necessary [17].

\subsection{Duality Transformation}

Based on work by Slemon [46], topologically-correct equivalent circuit models can be derived from magnetic circuit models using the principle of duality, with the duality transformation being directly performed as a topological exercise. This type of model includes the effects of saturation in each individual leg of the core as well as leakage effects.

Table 2.1 lists the duality pairs for the transformation. A duality transformation example for the single-phase shell-form transformer with concentric windings of Figure 2.1 is given in Figures 2.5 and $2.6[7,13,46]$.

Table 2.1 Duality Transformation

\begin{tabular}{|c|c|c|}
\hline Magnetic Circuit & Electric Circuit & Remark \\
\hline Meshes & Nodes & \\
\hline Nodes & Meshes & $M M F=N^{*} \boldsymbol{i}$ \\
\hline MMF & $\boldsymbol{i}$ (current) & $v=d \lambda d t$ \\
\hline$d \lambda / d t$ & $\boldsymbol{v}$ (voltage) & $L=N^{2} / \mathscr{R}$ \\
\hline $\mathfrak{R}$ (reluctance) & $\boldsymbol{L}$ (inductance) & \\
\hline Series & Parallel & \\
\hline Parallel & Series & \\
\hline
\end{tabular}




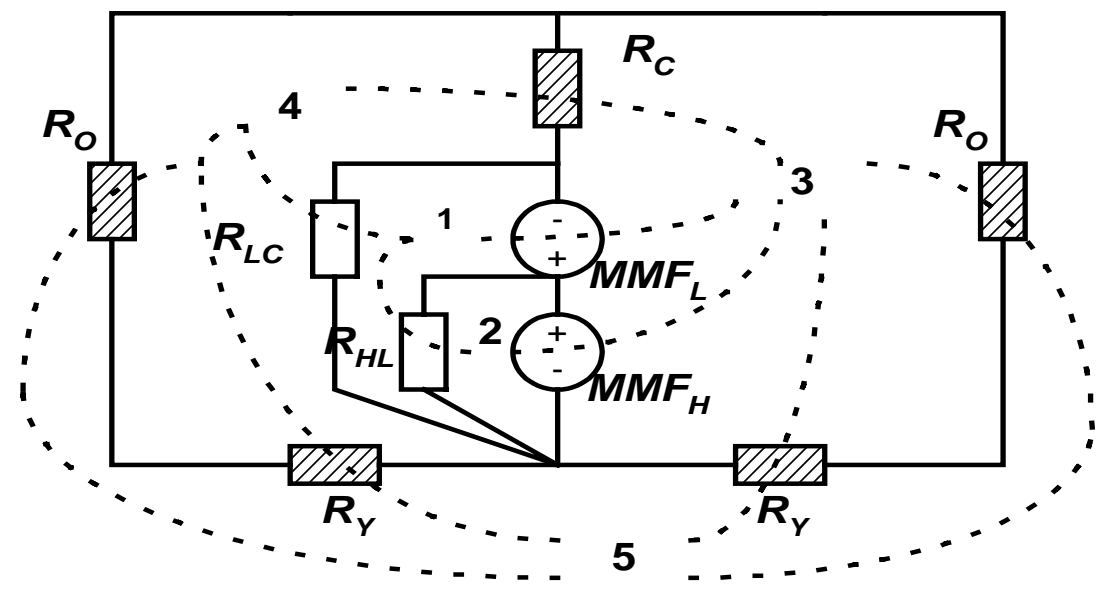

Figure 2.5 Equivalent Magnetic Circuit and Topological Development

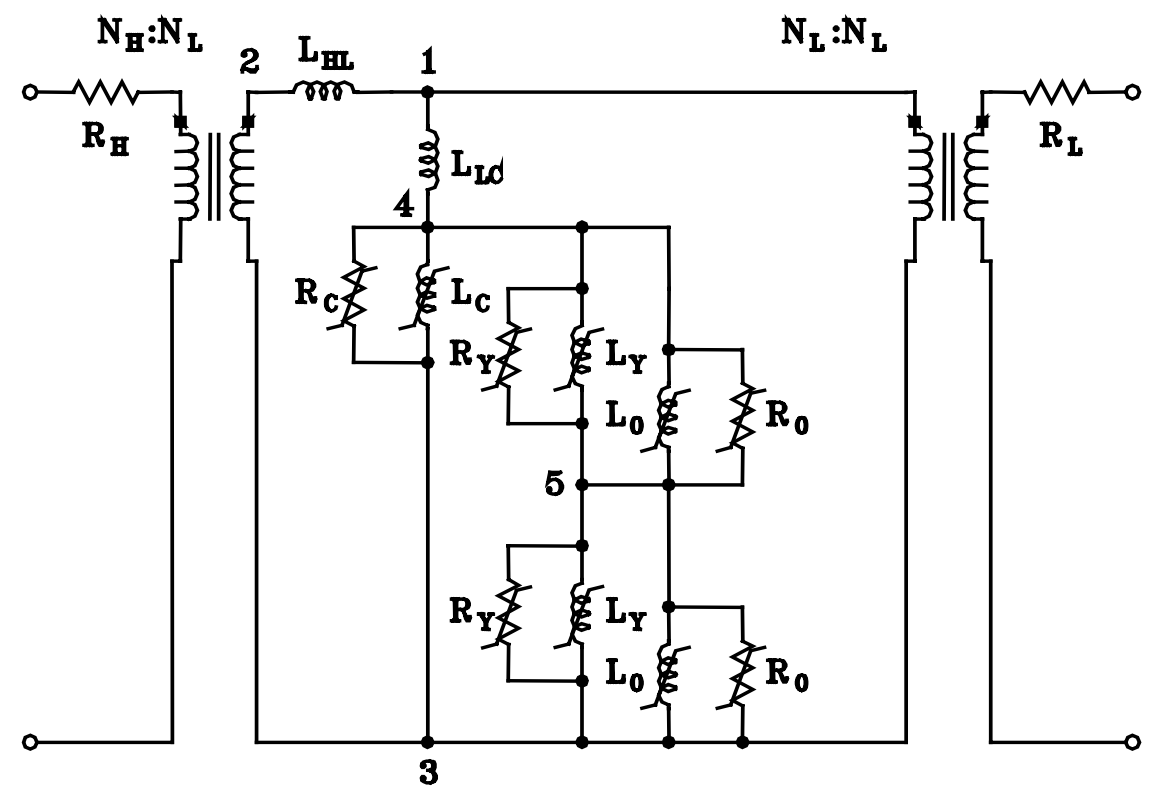

Figure 2.6 Equivalent Electrical Circuit Derived from Duality Transformation

Core sections are labeled as $\mathrm{C}$ for center leg, $\mathrm{O}$ for outer legs, and Y for yokes. $\Phi_{\mathrm{HL}}$ is the leakage flux that is assumed to flow between the high and the low voltage coils, and $\Phi_{\mathrm{LC}}$ is the leakage flux between the low voltage coil and the core. The next step is to convert the distributed magnetic circuit into a lumped parameter equivalent, as shown 
with solid lines in Figure 2.5. The electrical dual, shown with dashed lines, is then developed. An electrical node is placed in the center of each magnetic circuit mesh, as well as outside the circuit. Then, as shown in Table 2.1, each MMF source and reluctance is replaced by its electrical dual and connected between the neighboring nodes. To maintain mathematical duality, the polarity of the current source must be consistent with the MMF sources. The last step is to replace the current sources with ideal coupling transformers. In Figure 2.6, the core and leakage behaviors are electrically isolated from the external winding connections, which is an advantage for grounded or interconnected windings. Winding resistances are added to the high- and low-voltage windings. The five core sections in Figure 2.6 can be simplified in this case by combing them into one equivalent magnetizing inductance.

The equivalent circuits resulting from duality transformations are topologically correct lumped-parameter representations. Duality-derived models can be implemented with standard EMTP elements such as an ideal transformer, lumped RLC, or saturable inductor.

However, practical application of this model for a three-phase transformer has been hampered by a difficulty in obtaining the required model parameters. Factory test data provided by transformer manufacturers is not enough for this model. One particularly troublesome problem is that exciting currents are stated in RMS amperes and calculated as an average of the three phase currents. This is not enough to allow core parameters to be properly calculated, since the currents are not sinusoidal and not the same in every phase. 


\subsection{Coil/Winding Capacitance with Damping Resistance}

For transient studies that involve frequencies up to a few $\mathrm{kHz}$, stray capacitance of transformer coils must be added to the transformer model as shown Figure 2.7. Capacitances are actually distributed, but lumped parameters at the winding terminals for the total capacitance can be used with reasonable accuracy in this case. The capacitances represent the electric coupling between two windings of the same phase or between each winding and the earthed fittings of the transformer, i.e. the tank and the core $[1,26,50]$.

The effective terminal capacitance can be determined based on the frequency of oscillation of each winding by using Equations (2.10) through (2.13) [50].

Effective capacitance $C_{\text {eff }}=1 /\left[(2 \pi f)^{2} \cdot L\right]$

where f: TRV frequency of each winding in $\mathrm{Hz}$,

$L:$ transformer leakage inductance in $H, C$ : effective capacitance in $F$

Effective capacitance for the high-voltage winding $\quad C_{e f f}=C_{H}+C_{H L}$

Effective capacitance for the low-voltage winding $\quad C_{\text {eff }}=C_{L}+C_{H L}$

High-frequency capacitive coupling ratio $\quad C_{H L} /\left(C_{H L}+C_{L}\right)$

Representative frequencies for power transformers are reported by Harner and Rodriguez and the high-frequency capacitive coupling ratio is generally lower than 0.4 [50].

Due to high-frequency winding resistance and eddy current losses, the oscillations are damped. This damping is represented by the resistance to ground in the equivalent circuit shown in Figure 2.8. For most transformers, the damping is usually such that the 
damping factor, DF, (i.e., the ratio of successive peaks of opposite polarity in the oscillation) is on the order of 0.6 to 0.8 . Thus, the high-frequency damping resistance, RD, can be calculated using the equation given in Figure 2.7 [50].

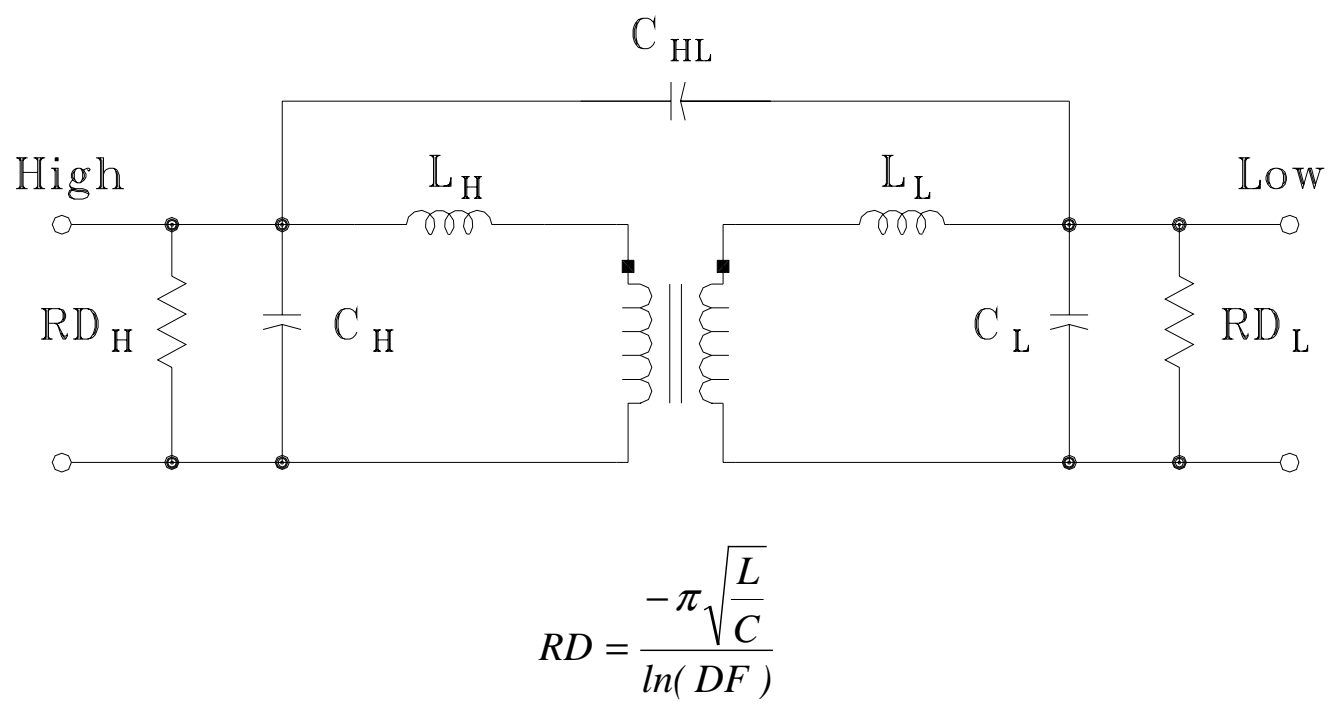

Figure 2.7 Equivalent Circuit for Capacitance with Damping Resistance

\subsection{Parameter Estimation using Engineering Optimization}

When developing a duality model for the equivalent circuit of a three-phase transformer in the EMTP, the main problem is the lack of reliable data from which to obtain the parameters of the equivalent circuit, i.e. leakage inductance, nonlinear magnetizing inductance for core saturation and nonlinear resistance for core loss. Thus, some parameter estimation methods might be used to build a topological model based on normally available test data. This parameter estimation problem is a nonlinear multivariable problem with equality and inequality constraints. Therefore, a nonlinear optimization strategy must be implemented for this case. 


\subsubsection{Engineering Optimization}

The application of optimization techniques in engineering can be found in many analysis problems arising in engineering model development. This parameter estimation problem inherently transforms to an optimization problem to determine the parameters of some semi-theoretical model given a set of test data, because the model parameters must be selected so that the model fits the data as closely as possible. A general formulation of nonlinear constrained optimization problem is given by [40]:

$$
\begin{aligned}
& \text { Minimize } F(\boldsymbol{x}) \quad \text { for } \boldsymbol{x}=\left(x_{1}, x_{2}, \ldots, x_{N}\right) \\
& \text { subject to } g_{j}(\boldsymbol{x}) \geq 0 \text { for } j=1,2, \ldots, J \text { and } h_{k}(\boldsymbol{x})=0 \text { for } k=1,2, \ldots K \\
& \text { where, } \boldsymbol{x} \text { variables (a set of design parameters) } \\
& F(\boldsymbol{x}) \text { : objective functions to be minimized } \\
& g_{j}(\boldsymbol{x}) \text { : inequality constraints } \\
& h_{k}(\boldsymbol{x}) \text { : equality constraints }
\end{aligned}
$$

The determination of the parameters might be carried out applying the strategy of minimizing the sum of quadratic errors of the approximate values with respect to the exact values.

$$
\begin{aligned}
& F(x)=\sum_{i=1}^{N}\left[y_{i}-f\left(x, \theta_{i}\right)\right]^{2} \\
& \text { Where, } y_{i}: \text { test data at the test condition } \theta_{i} \\
& f\left(x, \theta_{i}\right): \text { predicted value at the test condition } \theta_{i}
\end{aligned}
$$

The difference $y_{i}-f\left(\boldsymbol{x}, \theta_{i}\right)$ between the test data $y_{i}$ and the predicted value $f\left(\boldsymbol{x}, \theta_{i}\right)$ measures how close the prediction is to the test data and is called the residual. The sum of the squares of the residuals at all the test points gives an indication of goodness of the fit. 
This data-fitting problem can thus be viewed as optimization problem in which $F(\boldsymbol{x})$ is minimized by appropriate choice of $\boldsymbol{x}$.

The challenges in the unconstrained optimization approach of the Equation (2.15) are spurious solutions like "local optima" that merely satisfy the requirements on the derivatives of the functions without constraints. Therefore, a constrained optimization approach may be appropriate for parameter estimation of transformer model.

As the necessary conditions of optimality for equality-constrained problems are Lagrange multipliers, the necessary conditions of optimization problems with equality and inequality constraints are Kuhn-Tucker conditions:

$$
\begin{aligned}
& \nabla F(x)-\sum_{j=1}^{J} u_{j} \nabla g_{j}(x)-\sum_{k=1}^{K} v_{k} \nabla h_{k}(x)=0 \\
& g_{j}(x) \geq 0 \text { for } j=1,2, \ldots, J \text { and } h_{k}(x)=0 \text { for } k=1,2, \ldots K \\
& u_{j} g_{j}(x)=0 \quad \text { and } u_{j} \geq 0 \text { for } j=1,2, \ldots, J
\end{aligned}
$$

Where, $\nabla F(x): N$-component column vector of first derivatives of $F(\boldsymbol{x})$ $\nabla g_{j}(\boldsymbol{x}): J$-component column vector of first derivatives of $g_{j}(\boldsymbol{x})$ $\nabla h_{K}(\boldsymbol{x}): K$-component column vector of first derivatives of $h_{k}(\boldsymbol{x})$ $u_{j}$ : Lagrange multiplier corresponding to contraint $g_{j}(\boldsymbol{x})$ $v_{k}$ : Lagrange multiplier corresponding to contraint $h_{k}(\boldsymbol{x})$

The solutions of Kuhn-Tucker conditions form the basis of many nonlinear programming algorithms, which attempt to directly compute the Lagrange multiplier.

There are many strategies for engineering optimization. For unconstrained optimization, methods can be broadly categorized in terms of the derivative information. 
Search methods that do not require gradients or other derivative information and use only function evaluations are most suitable for the problems that are nonlinear or have a number of discontinuities. One typical numerical search method is simplex search method.

Gradient methods are generally efficient when the function to be minimized is continuous in its first derivative. Gradient methods use information about the slope of the function $\nabla \mathrm{F}(\mathrm{x})$ to dictate a direction of search where the minimum is thought to lie. Of the methods that use gradient information, there are the quasi-Newton methods or the Conjugate Gradient methods. Quasi-Newton methods only require differences of gradients of the Lagrangian function. The gradient information is either supplied through analytically calculated gradients, or derived by a numerical differentiation method.

Higher order methods, such as Newton's methods, are only really suitable when the second order information is readily and easily calculated since calculation of the second order information, using numerical differentiation, is computationally expensive.

There are strategies for exploiting linear approximations to nonlinear problems like feasible direction methods, successive linear approximation methods, quadratic approximation methods or constrained variable metric methods.

There are a number of different optimization strategies. An efficient and accurate solution to a given optimization problem is not only dependent on the size of the problem in terms of the number of constraints and design variables but also on characteristics of the objective function and constraints. 


\subsubsection{Applicable Methods in MATLAB ${ }^{\circledR}$}

MATLAB $^{\circledR}$ Optimization tool box a collection of functions for many types of optimization such as nonlinear minimization, quadratic and linear programming, nonlinear least squares and curve-fitting, and nonlinear system of equation solving, and etc..

For the paramter estimation in this work, the constrained nonlinear minimization, nonlinear least squares, and curve-fitting techiques are necessary.

One of constrained nonlinear minimization functions in the MATLAB ${ }^{\circledR}$ Optimization tool box is "fmincon". This function solves a constrained nonlinear multivariable problem.

$$
x=\operatorname{fmincon}\left(f u n, X_{0}, A, b, A_{e q}, b_{e q}, l_{b}, u_{b}, \text { nonlcon }\right)
$$

"fmincon" finds the constrained minimum of a scalar function of several variables starting at an initial estimate $\mathrm{X}_{0}$. This is referred to as constrained nonlinear optimization or nonlinear programming. It finds x to minimizes "fun" subject to the linear equalities $\mathrm{A}_{\mathrm{eq}} * \mathrm{X}=\mathrm{b}_{\mathrm{eq}}$ as well as the linear inequalities $\mathrm{A} * \mathrm{X}<=\mathrm{b}$. It subjects the minimization to the nonlinear inequalities $\mathrm{c}(\mathrm{X})<=0$ or nonlinear equalities $\mathrm{c}_{\mathrm{eq}}(\mathrm{X})=0$. fmincon uses a Sequential Quadratic Programming (SQP) method. In Sequential Quadratic Programming (SQP) method, a Quadratic Programming (QP) subproblem is solved at each iteration. An estimate of the Hessian of the Lagrangian is updated at each iteration. A line search is performed using a merit function [53]. 
One of the nonlinear least squares functions in MATLAB ${ }^{\circledR}$ Optimization tool box is "Isqnonlin".

$$
\left.x=\text { lsqnonlin(fun, } x_{0}\right)
$$

$x=$ lsqnonlin $\left(\right.$ fun,$\left.x_{0}\right)$ starts at the point $\mathrm{x}_{0}$ and finds a minimum to the sum of squares of the functions described in fun. fun should return a vector of values and not the sum-of-squares of the values. By default, lsqnonlin chooses the large-scale algorithm. This algorithm is a subspace trust region method and is based on the interior-reflective Newton method. lsqnonlin with options. LargeScale set to 'off' uses the LevenbergMarquardt method with line-search. Alternatively, a Gauss-Newton method with linesearch may be selected. 1sqnonlin does not handle equality constraints. The function to be minimized must be continuous. Isqnonlin only handles real variables. When $\mathrm{x}$ has complex variables, the variables must be split into real and imaginary parts [53].

One of the nonlinear curve-fitting (data-fitting) functions in MATLAB ${ }^{\circledR}$ Optimization tool box is "lsqcurvefit". This function solves nonlinear curve-fitting (datafitting) problems in the least squares sense.

$$
\left.x=\text { lsqcurvefit(fun, } x_{0}, x d a t a, y d a t a\right)
$$

With given input data xdata and observed output ydata, $x=l \operatorname{sqnonlin}\left(f u n, x_{0}\right)$ starts at the point $\mathrm{x}_{0}$ and finds coefficients $\mathrm{x}$ that "best-fit" the equation $\mathrm{F}(\mathrm{x}, \mathrm{xdata})$ where $\mathrm{xdata}$ and ydata are vectors and $\mathrm{F}(\mathrm{x}, \mathrm{xdata})$ is a vector valued function. The function lsqcurvefit uses the same algorithm as lsqnonlin. Its purpose is to provide an interface designed specifically for data-fitting problems. The function to be fit, fun is a function that takes a 
vector $\mathrm{x}$ and returns a vector $\mathrm{F}$, the objective functions evaluated at $\mathrm{x}$. The sum of squares should not be formed explicitly. Instead, the function returns a vector of function values.

The default line search algorithm is a mixed quadratic and cubic polynomial interpolation and extrapolation method. The function to be minimized must be continuous. 1sqcurvefit may only give local solutions. When $\mathrm{x}$ has complex variables, the variables must be split into real and imaginary parts [53]. 


\section{CHAPTER 3}

\section{THREE-PHASE TRANSFORMER MODEL}

This Chapter describes important parameters and the implementation of existing models, in order to gain insights on parameters. The pros and cons of the existing models are briefly discussed, along with some examples.

Detailed representation of a power transformer can be very complex due to the many variations in core and coil design and their complex behaviors during transient phenomena. The most suitable representation depends on several factors: the behavior being simulated, available data, and core design. One of several models valid for a specific frequency range may be used. According to CIGRE WG 33-02 [52], frequency ranges can be classified as four groups with some overlapping between them (Table 3.1).

In this work, transformer modeling for low-frequency and slow-front transients is considered. This is suitable for simulation of power system transients such as excitation inrush currents, ferroresonance, short circuits, abnormalities including transformer faults, and switching overvoltages.

An autotransformer is a transformer configuration that has part of its winding common to both the input and output, i.e. there is no electrical isolation. If the voltage ratio is favorable (in practice, typically $\leq 3: 1$ ), an autotransformer is advantageous from the point of view of the equivalent volt-amp rating. The effective increase in equivalent 
rating reduces the weight, the size, no-load loss, load losses and the short circuit impedance. The use of an autotransformer makes it possible for a high power rating to be constructed as a single unit three-phase transformer. In this work, the model for a threewinding autotransformer is considered.

To develop a model for a three-phase transformer, transformer physical design information and characteristic data are needed. However, it is most unusual to have a case where complete physical design information and dimensions are available. Utilities typically can't afford to take transformers out of service, don't have the equipment for taking field measurements, or can't afford the field crew to perform them. Often, all the information we will have is what is on the nameplate, or maybe the basic factory tests. Utilities have typically not had the foresight to request detailed tests, and the state of the art has not been advanced enough to know what tests or parameters to request as part of their purchase specification. Typically, factories have done only the minimum required compliance testing.

Typical transformer factory test reports available from manufacturers consist of data like Table 3.2, which summarizes the report given in Appendix C. The available data are no-load kW losses and true RMS exciting current at $100 \%$ and $110 \%$ of rated voltage. However, there is no information on transformer core type, core material, etc. It should be noted that the "RMS exciting current" taken from factory tests is actually the average of the three measured true RMS phase currents. Usually, zero sequence short-circuit tests are not performed, so that information is not available either. 
Table 3.1 CIGRE Modeling Recommendation for Power Transformer [52]

\begin{tabular}{|c|c|c|c|c|}
\hline $\begin{array}{c}\text { Parameter } \\
\text { /Effect }\end{array}$ & $\begin{array}{c}\text { Low Frequency } \\
\text { Transients }\end{array}$ & $\begin{array}{c}\text { Slow Front } \\
\text { Transients }\end{array}$ & $\begin{array}{c}\text { Fast Front } \\
\text { Transients }\end{array}$ & $\begin{array}{c}\text { Very Fast } \\
\text { Front } \\
\text { Transients }\end{array}$ \\
\hline $\begin{array}{c}\text { Short-circuit } \\
\text { impedance }\end{array}$ & Very important & Very important & Important & Negligible \\
\hline Saturation & Very important & Very important ${ }^{(1)}$ & Negligible & Negligible \\
\hline Iron losses & Important $^{(2)}$ & Important & Negligible & Negligible \\
\hline Eddy currents & Very important & Important & Negligible & Negligible \\
\hline $\begin{array}{c}\text { Capacitive } \\
\text { coupling }\end{array}$ & Negligible & Important & $\begin{array}{c}\text { Very } \\
\text { important }\end{array}$ & $\begin{array}{c}\text { Very } \\
\text { important }\end{array}$ \\
\hline
\end{tabular}

1) Only for transformer energization phenomena, otherwise important

2) Only for resonance phenomena

Table 3.2 Transformer Factory Test Data

\begin{tabular}{|r|c|c|}
\hline \multicolumn{3}{|c|}{$\begin{array}{c}\text { 345000 Grd.Y/118000 Grd.Y/13800 Delta, } \\
\text { 3-phase auto-transformer @OA/FOA/FOA }\end{array}$} \\
\cline { 2 - 3 } H- 296/394/490MVA, X-296/394/490MVA, Y-77/103/128MVA \\
\hline \multirow{2}{*}{ Open-Circuit Test } & Exciting Current & No Load Loss \\
\cline { 2 - 3 } & $0.76 \% @ 100 \%$ Voltage & 297.6kW @ 100\%Voltage \\
\hline Short-Circuit Test & I.71\%@110\%Voltage & 402.24kW @110\%Voltage \\
\hline H-X & $6.21 \% @$ @296MVA & Load Loss \\
\hline H-Y & $55.9 \% @ 296 \mathrm{MVA}$ & $258.76 \mathrm{~kW} @ 77 \mathrm{MVA}$ \\
\hline $\mathrm{X}-\mathrm{Y}$ & $42.1 \% @ 296 \mathrm{MVA}$ & $237.68 \mathrm{~kW} @ 77 \mathrm{MVA}$ \\
\hline
\end{tabular}

\subsection{STC Model}

A more correct model of a three-phase autotransformer can be obtained by representing high $(\mathrm{H})$ and low $(\mathrm{X})$ voltage terminals with the actual series winding $(\mathrm{S})$ and common winding (C) as shown in Figure 3.1. This requires a re-definition of the short-circuit data in terms of windings $\mathrm{S}$ and C. Since most autotransformers have a tertiary winding, this winding $\mathrm{T}$ is included in the re-definition. The autotransformer can 
therefore be represented as a transformer with the 3 windings S, C, and T. The voltage ratings are $\mathrm{V}_{\mathrm{S}}=\mathrm{V}_{\mathrm{H}}-\mathrm{V}_{\mathrm{X}}, \mathrm{V}_{\mathrm{C}}=\mathrm{V}_{\mathrm{X}}, \mathrm{V}_{\mathrm{T}}=\mathrm{V}_{\mathrm{Y}}$. This modification can be explained in terms of the equivalent star-circuit of Figure 3.1, with the impedances $Z_{S}, Z_{C}, Z_{T}$ based on $V_{S}, V_{C}$, $\mathrm{V}_{\mathrm{T}}$.

To learn the details of the Saturable Transformer model, one was implemented and benchmarked against factory test reports using the data of Table 3.2. The comparison is shown in Table 3.3. Equivalent Impedances modified for this model are $\mathrm{N}=2.924$, $\mathrm{Z}_{\mathrm{SC}}=14.344 \%, \mathrm{Z}_{\mathrm{CT}}=42.1 \%, \mathrm{Z}_{\mathrm{TS}}=67.98 \%, \mathrm{Z}_{\mathrm{S}}=20.112 \%(11.67 \Omega), \mathrm{Z}_{\mathrm{C}}=-5.768 \%(-0.9044$ $\Omega), Z_{\mathrm{T}}=47.868 \%(0.9239 \Omega)$ at 296-MVA using each winding's voltage base.

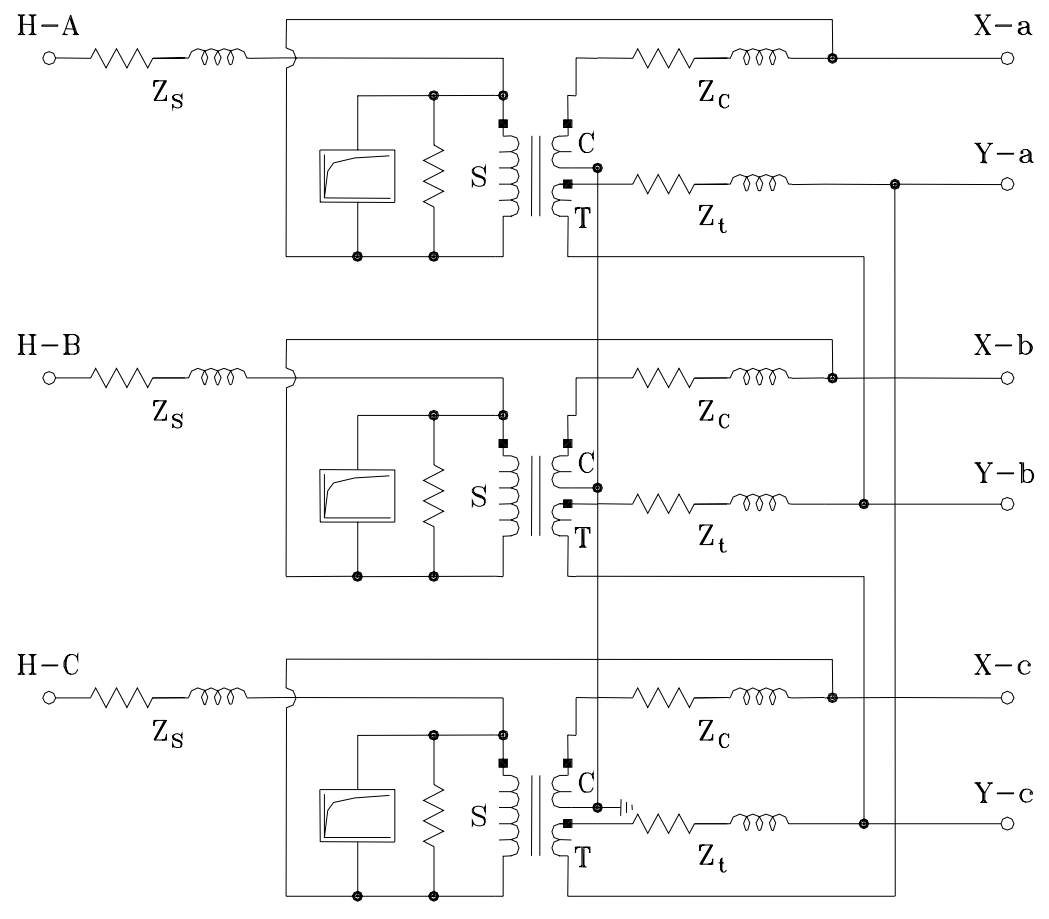

Figure 3.1 STC Model for Three-phase Three-winding Autotransformer [6] 
This model is limited to three-winding three-phase banks of single-phase units and may be numerically unstable because of negative short-circuit inductance in the equivalent circuit $[6,40]$. Also the attachment point of core equivalent is not topologically correct.

Table 3.3 Comparisons of STC Model with Test Report

\begin{tabular}{|c|c|c|}
\hline \multicolumn{3}{|c|}{ Model: 345000 Grd.Y/ 118000 Grd.Y/ 13800 Delta - 296MVA @ OA } \\
\hline & Test Report & STC Model \\
\hline \multirow{6}{*}{$\begin{array}{c}\text { Open- } \\
\text { Circuit } \\
\text { Test }\end{array}$} & \multicolumn{2}{|c|}{ Exciting Current @ 345kV Side } \\
\hline & 3.76Amp.RMS@100\%Voltage & 3.77Amp.RMS, 5.33Amp.peak, \\
\hline & 8.47Amp.RMS@110\%Voltage & $\begin{array}{c}\text { 6.92Amp.RMS, 11.98Amp.peak } \\
\text { @110\%Voltage }\end{array}$ \\
\hline & \multicolumn{2}{|c|}{ No Load Loss per Phase } \\
\hline & 99.20kW@100\%Voltage & 99.82kW@100\%Voltage \\
\hline & 134.08kW@110\%Voltage & 120.78kW@110\%Voltage \\
\hline \multirow{8}{*}{$\begin{array}{c}\text { Short- } \\
\text { Circuit } \\
\text { Test }\end{array}$} & \multicolumn{2}{|c|}{ Short-Circuit Current } \\
\hline & 700.53Amp.peak & 700.55Amp.peak \\
\hline & 182.23Amp.peak & 182.53Amp.peak \\
\hline & 532.80Amp.peak & 532.91Amp.peak \\
\hline & \multicolumn{2}{|c|}{ Load Loss per Phase } \\
\hline & P-S 126.31kW @296MVA & $126.61 \mathrm{~kW}$ \\
\hline & P-T 86.25kW@77MVA & $86.29 \mathrm{~kW}$ \\
\hline & S-T 79.227kW@77MVA & $79.21 \mathrm{~kW}$ \\
\hline
\end{tabular}

\subsection{BCTRAN Model}

BCTRAN models were next investigated. This model is a more stable model for multi-winding transformers than the STC model, but permits only linear magnetizing branches to be incorporated in the matrix. Note that this model is of particular interest since it was implemented in a transient investigation study [14], where deficiencies in transformer representation were one of the motivations for this work. The overall model 
implanted in that case used BCTRAN for short-circuit representation, with an externallyattached simplistic core model, as shown in Figure 3.2.

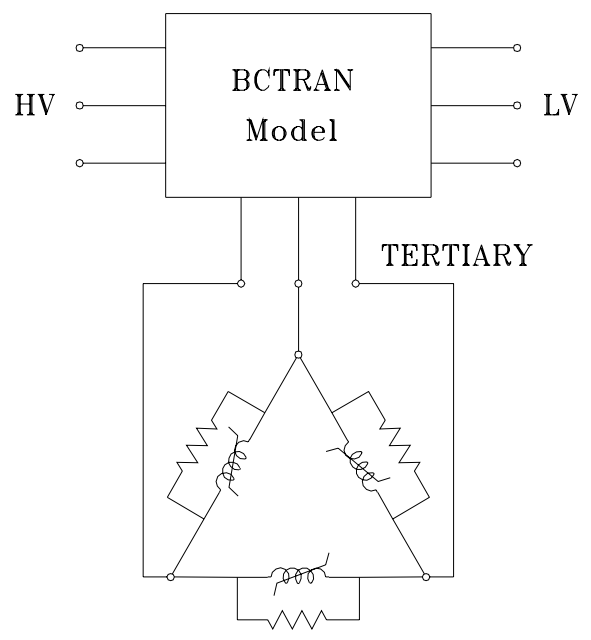

\section{Figure 3.2 BCTRAN Model with External Core Elements \\ for Three-phase Three-winding Autotransformer}

Core and load losses from the test data in Table 3.2 are employed to calculate the model parameters. To verify the transformer model developed using BCTRAN, results from simulated open and short circuit tests were compared to the transformer test report. The comparison is shown in Table 3.4. To model the magnetic core saturation and losses of the transformer, core effects are omitted in the BCTRAN model and replaced by external nonlinear elements. Core magnetization and losses are attached on the tertiary terminals as a nonlinear inductance in parallel with a linear resistor, as shown in Figure 3.2. Using the $100 \%$ and $110 \%$ excitation data from the factory test report, the RMS magnetizing current is obtained by removing the core loss component from the exciting current as Equation (3.1).

$$
I_{r m s}=\sqrt{I_{e x c}^{2}-I_{\text {core }}^{2}}
$$


Table 3.4 Comparisons of BCTRAN Model with Test Report

\begin{tabular}{|c|c|c|}
\hline \multicolumn{3}{|c|}{ Model: 345000 Grd.Y/ 118000 Grd.Y/ 13800 Delta - 296MVA @ OA } \\
\hline & Test Report & BCTRAN Model \\
\hline \multirow{6}{*}{$\begin{array}{c}\text { Open- } \\
\text { Circuit } \\
\text { Test }\end{array}$} & \multicolumn{2}{|c|}{ Exciting Current @345kV Side } \\
\hline & 3.76Amp.RMS@100\%Voltage & 3.75Amp.RMS, 5.30Amp.peak, \\
\hline & 8.47Amp.RMS@110\%Voltage & $\begin{array}{c}\text { 7.37Amp.RMS, 13.65Amp.peak } \\
@ 110 \% \text { Voltage }\end{array}$ \\
\hline & \multicolumn{2}{|c|}{ No Load Loss per Phase } \\
\hline & 99.20kW@100\%Voltage & 98.44kW@100\%Voltage \\
\hline & 134.08kW@110\%Voltage & 119.11kW@110\%Voltage \\
\hline \multirow{8}{*}{$\begin{array}{c}\text { Short- } \\
\text { Circuit } \\
\text { Test }\end{array}$} & \multicolumn{2}{|c|}{ Short-Circuit Current } \\
\hline & 700.53Amp.peak & 700.65Amp.peak \\
\hline & 182.23Amp.peak & 182.23Amp.peak \\
\hline & 532.80Amp.peak & 532.81Amp.peak \\
\hline & \multicolumn{2}{|c|}{ Load Loss per Phase } \\
\hline & P-S 126.31kW@296MVA & $126.51 \mathrm{~kW}$ \\
\hline & P-T 86.25kW@77MVA & $86.25 \mathrm{~kW}$ \\
\hline & S-T 79.227kW@77MVA & $79.230 \mathrm{~kW}$ \\
\hline
\end{tabular}

The resulting model represents all phase-to-phase coupling. However, it is valid only for the frequency at which the nameplate data was obtained. It models the terminal characteristics and does not consider differences in core or winding topology. Threelegged cores, five-legged cores, wye windings, delta windings, or autotransformer connections all get the same mathematical treatment. 


\subsection{Duality-Based Model}

Detailed models incorporating core nonlinearities can be derived by applying the principle of duality on topology-based magnetic models. This approach is very useful for creating models accurate enough for low-frequency transients. If capacitive effects are added, slow front transients can be adequately modeled.

The mesh and node equations of the magnetic circuit are the duals of the electrical equivalent's node and mesh equations respectively. The duality transformation can be directly performed as a topological exercise. The duality transformation for the threephase three-winding transformer in Table 3.4 is given in Figures 3.3 through 3.5. Details follow.

A three-winding three-leg core-type transformer is considered. Core sections are labeled as L for each leg, Y for each yoke. $\Phi_{\mathrm{SC}}$ is the leakage flux that is assumed to flow between the series and common windings, and $\Phi_{\mathrm{CT}}$ is the leakage flux between the common winding and the tertiary winding, $\Phi_{\mathrm{TL}}$ is the leakage flux between the tertiary winding and the core. The next step is to convert the distributed magnetic circuit into a lumped parameter equivalent, shown in solid lines in the center of Figure 3.4. The electrical dual, shown in dashed lines, is then developed. As shown in Figure 3.5, each MMF source and reluctance is replaced by its electrical dual and connected between the neighboring nodes. 
Table 3.4 Comparisons of Electrical and Magnetic Quantities

\begin{tabular}{|c|c|c|}
\hline Magnetic Circuit & Electric Circuit & Remark \\
\hline$m m f(\mathfrak{I}, A-t)$ & $v($ voltage,$V)$ & $m m f=N^{*} i=H^{*} I$ \\
\hline $\operatorname{Flux}(\phi, W b)$ & $i$ (current, A) & $\phi,=B * A$ \\
\hline$\Re\left(\right.$ reluctance, $\left.H^{-1}\right)$ & $R($ Resistance,$\Omega)$ & $L=N^{2} / \mathscr{R}$ \\
\hline $\begin{array}{c}\text { Magnetic field intensity } \\
(H, A-t / m)\end{array}$ & $\begin{array}{l}\text { Electric field intensity } \\
(E, V / m)\end{array}$ & \\
\hline Flux density $(B, T)$ & Current density $\left(\mathrm{J}, \mathrm{A} / \mathrm{m}^{2}\right)$ & \\
\hline Permeability $(\mu, \mathrm{H} / \mathrm{m})$ & Conductivity $(\sigma, S / m)$ & $\mu=B / H$ \\
\hline $\mathfrak{I}=\phi \mathfrak{R}$ & $\mathrm{V}=\mathrm{iR}$ & \\
\hline$B=\mu H$ & $J=\sigma E$ & \\
\hline $\begin{array}{c}\text { Flux linkage } \\
(\lambda, W b-t)=N \phi\end{array}$ & $N i($ A turn $)$ & \\
\hline$\Re=/ /(\mu A)=1 / P=1 / L$ & $R=l /(\sigma A)=1 / G$ & $L$ (inductance) \\
\hline
\end{tabular}

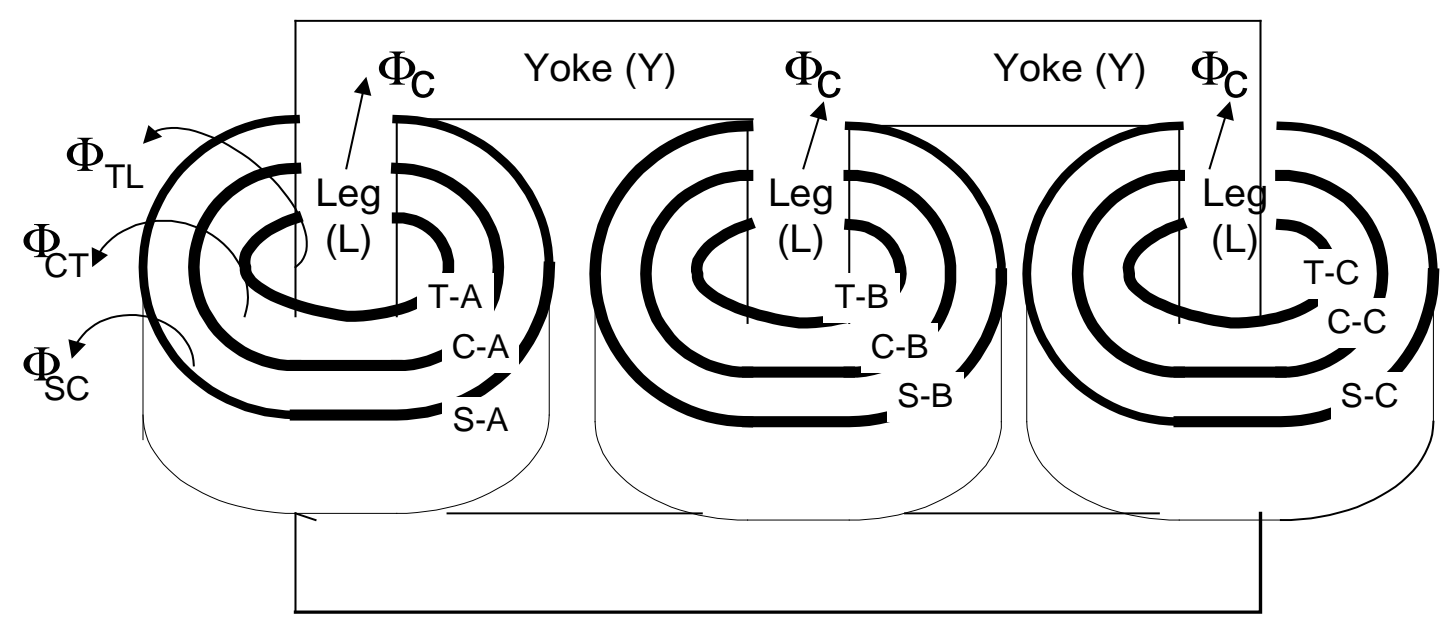

Figure 3.3 Three-phase Three-leg Core-type Transformer Structure 


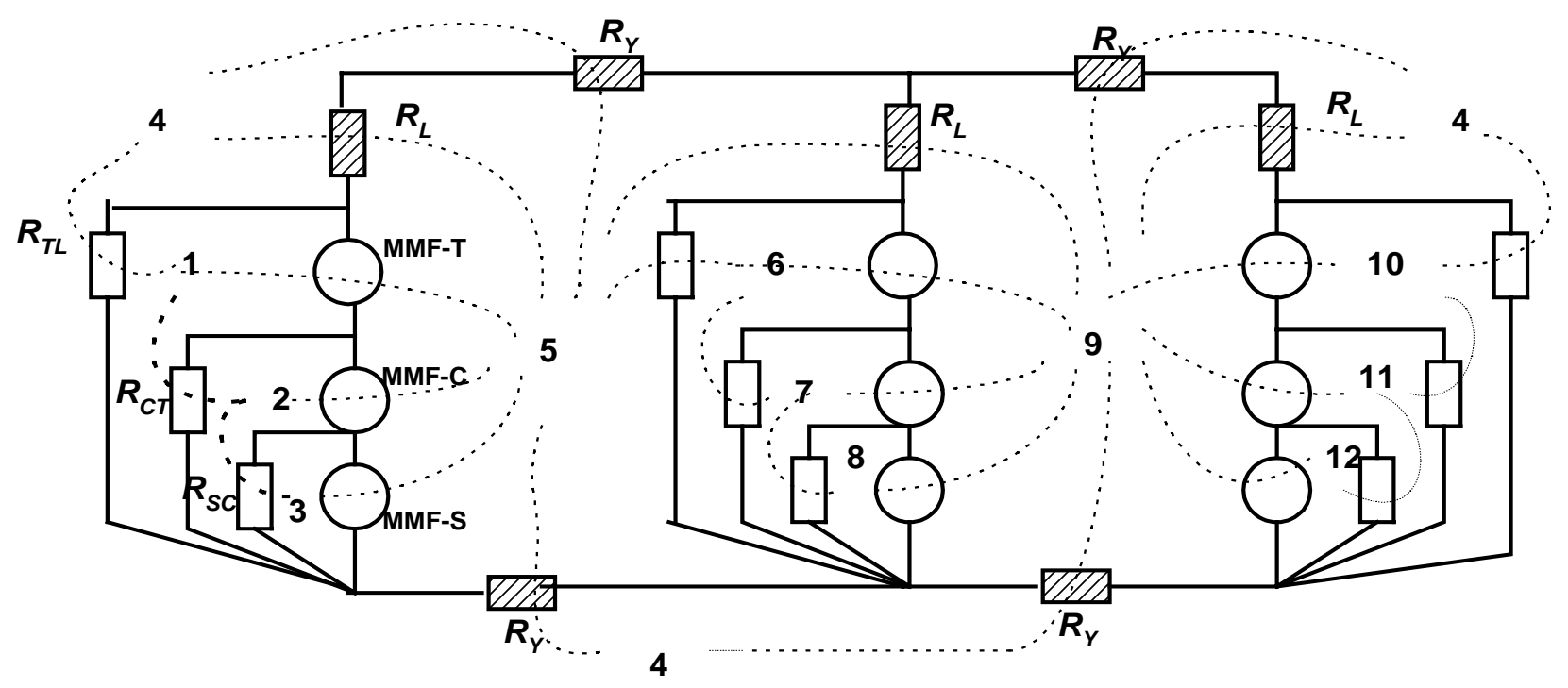

Figure 3.4 Duality Transformation
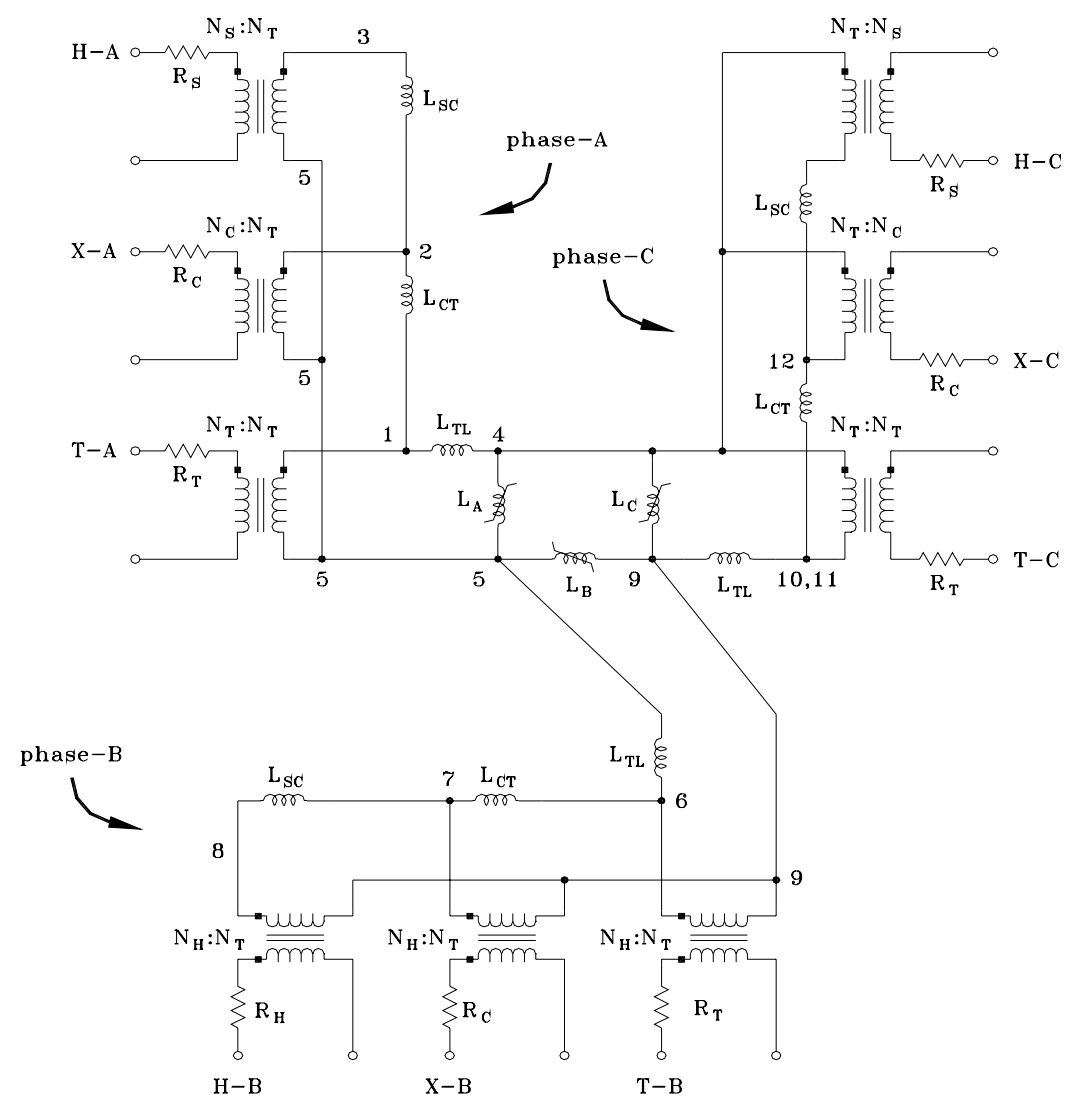

Figure 3.5 Equivalent Electric Circuit Derived from Duality Transformation 


\section{CHAPTER 4 \\ PARAMETERS FOR TRANSFORMER MODEL}

\subsection{Frequency-Dependency of Coil Resistance}

Coil resistances vary widely depending on the frequency of the current flowing. The variation is due to skin effect and proximity effect. Skin effect is caused by the nonuniform distribution of current in the conductor. As the frequency of the current is increased, more current flows near the surface of the conductor. Thus, the effective resistance increases. The effective resistance typically varies as the square root of frequency $[7,22]$.

$$
R_{a c}(f)=R_{60} \cdot\left[\frac{f}{60}\right]^{k} \text { where, } k \text { : about } 0.5, R_{60}: 60 \mathrm{~Hz} \text { resistance }
$$

However, a higher number of layers in the coil lead to a great resistance variation due to proximity effect. From reference [44], the frequency dependency of coil resistance is:

$$
\begin{aligned}
& R_{a c}(f)=\operatorname{real}\left[R_{D C} \cdot u \cdot\left(\operatorname{coth}(u)-\frac{2}{3} \cdot \tanh (u)+\frac{2}{3} \cdot n l^{2} \cdot \tanh \left(\frac{u}{2}\right)\right)\right] \\
& \delta=\sqrt{\frac{1}{\pi \cdot f \cdot \sigma \cdot \mu_{o}}} \quad \text { and } \quad u=(1+j) \cdot \frac{a}{\delta}
\end{aligned}
$$

where $a=$ coil diameter $(m), \delta=$ skin depth $(m), \mu_{0}=$ permeability of $\mathrm{Cu}\left(4 \pi \times 10^{-7}\right)$,

$$
\sigma=\text { conductance of } \mathrm{Cu}\left(0.5 \times 10^{8}\right), n l=\text { the number of layers }
$$

The effective resistance or the ratio of $\mathrm{R}(\mathrm{f}) / \mathrm{R}_{\mathrm{DC}}$ for the case of $\mathrm{a}=3 \mathrm{~mm}$ is given in Figure 4.1. In the case of one layer, the ratio of $\mathrm{R}(\mathrm{f}) / \mathrm{R}_{\mathrm{DC}}$ is almost the same as the square 
root of $\mathrm{f} / 60 \mathrm{~Hz}$ by the skin effect. In case of a multi-layer coil, the slope of $\mathrm{R}(\mathrm{f}) / \mathrm{Rdc}$ is almost the same as that of the skin effect in the range of $3 \mathrm{kHz}$ to $10 \mathrm{kHz}$. However, due to the proximity effect, the variation of $\mathrm{R}(\mathrm{f}) / \mathrm{R}_{\mathrm{DC}}$ is significantly greater in the range of $100 \mathrm{~Hz}$ to 3 $\mathrm{kHz}$. Figure 4.1 shows that the effective resistance of a winding with ten layers is almost the same as those of typical transformers in [17].

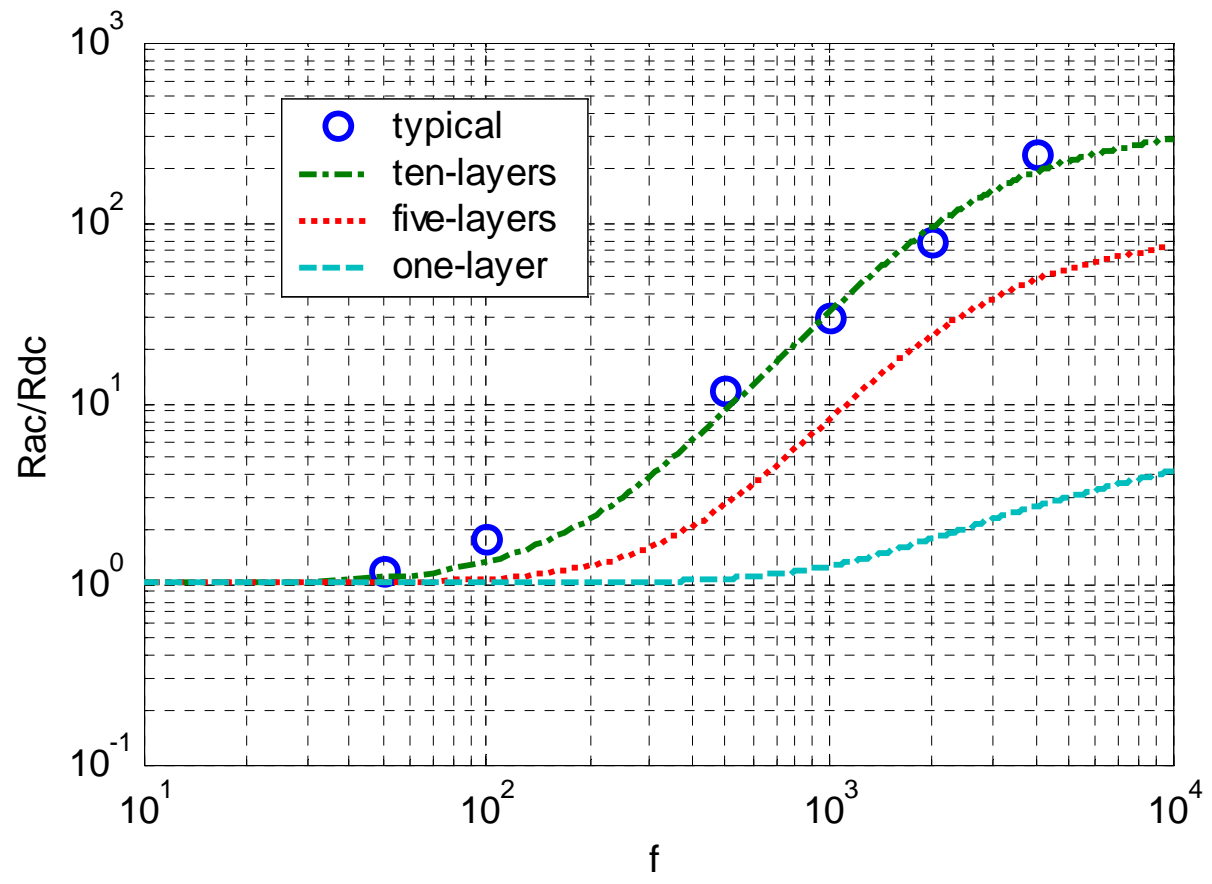

Figure 4.1 Effective Resistance at $\mathrm{a}=3 \mathrm{~mm}$

In case detailed data for the number of layers or winding size is not available, the $\mathrm{L} / \mathrm{R}$ ratio of the short-circuit impedance of typical transformers can be used to estimate the frequency-dependency of coil resistance. From Chapter 2 of the EMTP Theory Book [17], $\mathrm{L} / \mathrm{R}$ ratios of the short-circuit impedance of typical transformers are given for ratings of 20 MVA 500 MVA and frequency range of $50 \mathrm{~Hz} \sim 6000 \mathrm{~Hz}$. This is presented in Figures 4.2 and 4.3. Figure 4.3 shows that $K$ in Equation (4.1) is about 1.5 for the given frequency range. 


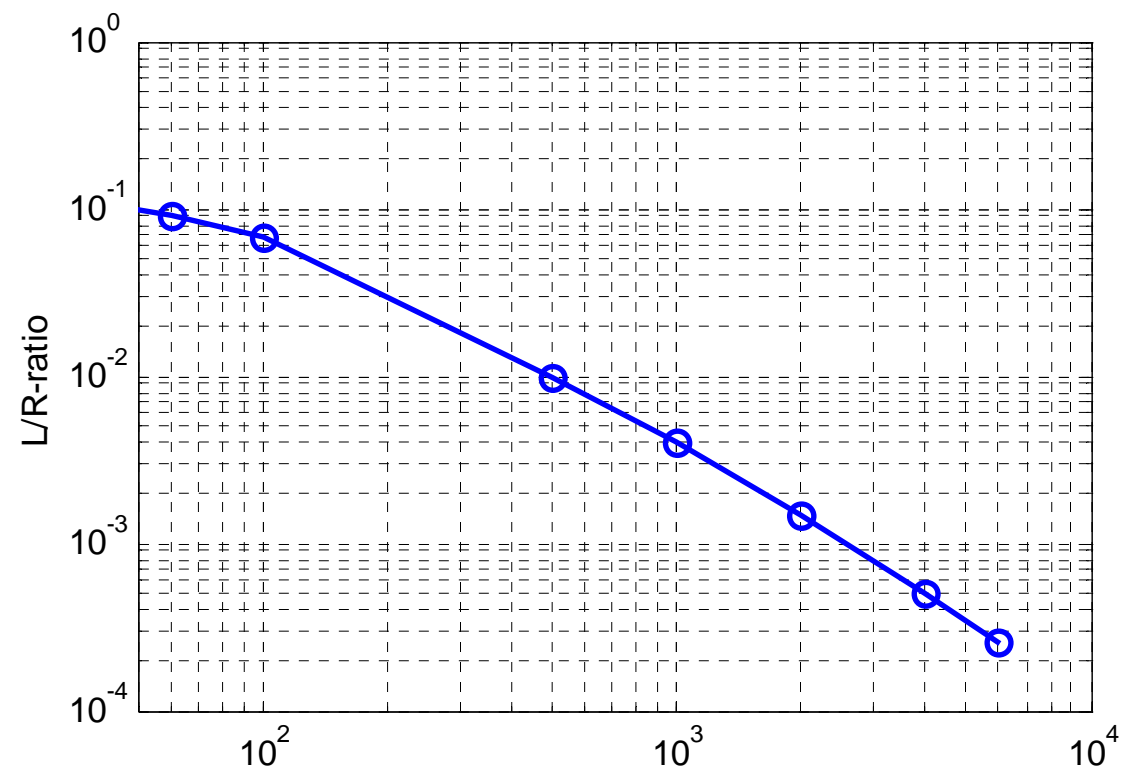

Figure 4.2 Typical L/R for Large Power Transformer [18]

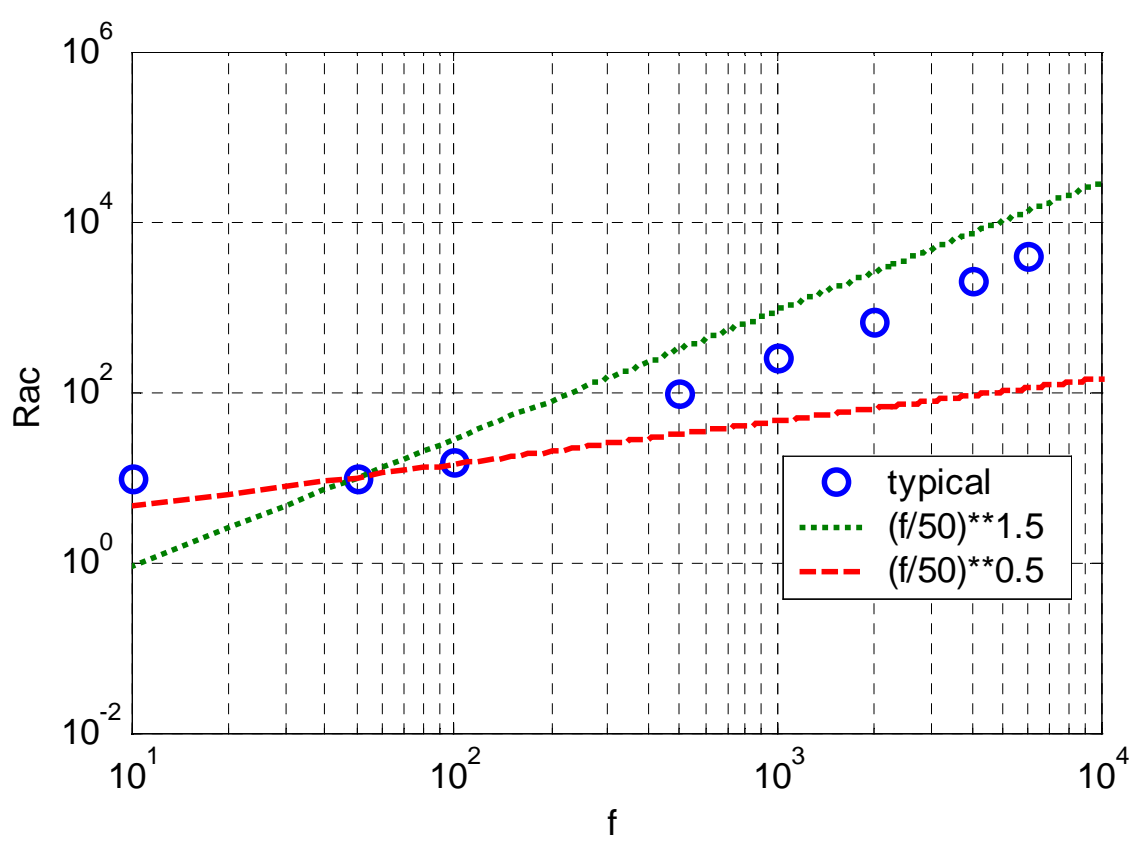

Figure 4.3 Typical Slope of Effective Resistance for Large Power Transformer [17] 
Table 4.1 Typical Effective Resistances for Large Power Transformer [17]

\begin{tabular}{|c|c|c|c|c|c|c|c|c|c|}
\hline $\mathrm{f}(\mathrm{Hz})$ & 0 & 50 & 60 & 100 & 500 & 1000 & 2000 & 4000 & 6000 \\
\hline $\mathrm{f} / 50$ & 0 & 1 & 1.2 & 2 & 10 & 20 & 40 & 80 & 120 \\
\hline $\mathrm{L} / \mathrm{R}_{\mathrm{eff}}$ & .106 & .1 & .091 & .0667 & .01 & 0.004 & .0015 & .0005 & .00026 \\
\hline $\mathrm{L}(\mathrm{H})$ & 1 & 1 & 1 & 1 & 1 & 1 & 1 & 1 & 1 \\
\hline $\mathrm{R}_{\text {eff }}(\Omega)$ & 9.4 & 10 & 11 & 15 & 100 & 250 & 670 & 2000 & 3900 \\
\hline $\begin{array}{c}\mathrm{R}_{\text {eff }} \\
(\mathrm{pu} @ 50 \mathrm{~Hz})\end{array}$ & .94 & 1 & 1.1 & 1.5 & 10 & 25 & 67 & 200 & 390 \\
\hline $\begin{array}{c}\mathrm{R}_{\text {eff }} \\
(\mathrm{pu} @ 60 \mathrm{~Hz})\end{array}$ & 0.83 & .88 & 1.0 & 1.364 & 9.091 & 22.73 & 60.91 & 181.8 & 354.5 \\
\hline
\end{tabular}

It is possible to represent the frequency-dependency of R using a Foster circuit as shown in Figure 4.4. If $L$ (leakage inductance) is given as $1 \mathrm{H}, \mathrm{Rp}$ is about $158 \mathrm{k} \Omega$ (from $\mathrm{L} / \mathrm{R}_{\text {eff }}$ ratio $=0.004$ at $1,000 \mathrm{~Hz}$ ) and $\mathrm{R}_{\mathrm{S}}$ is about $9.4 \Omega$ (from $\mathrm{L} / \mathrm{R}_{\text {eff }}$ ratio= 0.1 at $50 \mathrm{~Hz}$ ) by:

$$
\begin{aligned}
& R_{P}=\left(L / R_{e f f}(\omega)\right) \cdot \omega^{2} \cdot L \\
& R_{S}=R_{\text {eff }}(\omega)-R_{P} *\left(\omega^{*} L\right)^{2} /\left(R_{P}{ }^{2}+\left(\omega^{*} L\right)^{2}\right)
\end{aligned}
$$

If $\mathrm{R}_{\mathrm{S}}$ (DC resistance) is given as $9.4 \Omega, \mathrm{R}_{\mathrm{P}}$ is about $164 \mathrm{k} \Omega$ from $L / \mathrm{R}_{\text {eff }}$ ratio=0.004 and $\mathrm{R}_{\text {eff }}=250 \Omega$ at $1,000 \mathrm{~Hz}$ by below equation. However, this method produces correct value only at $50 \mathrm{~Hz}$ and at $1,000 \mathrm{~Hz}$ as shown in Figure 4.5 .

$$
R_{P}=\frac{\frac{(\omega L)^{2}}{\left(R_{e f f}-R_{S}\right)}+\sqrt{\frac{(\omega L)^{4}}{\left(R_{e f f}-R_{S}\right)^{2}}-4 \cdot \omega L}}{2}
$$




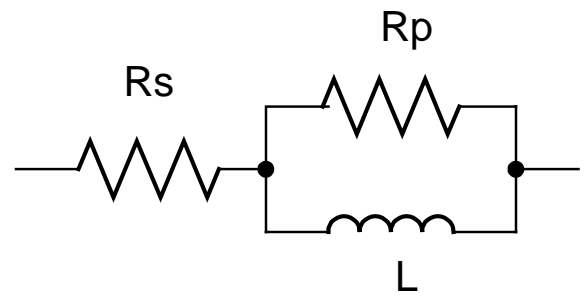

Figure 4.4 Foster Circuit with One Cell

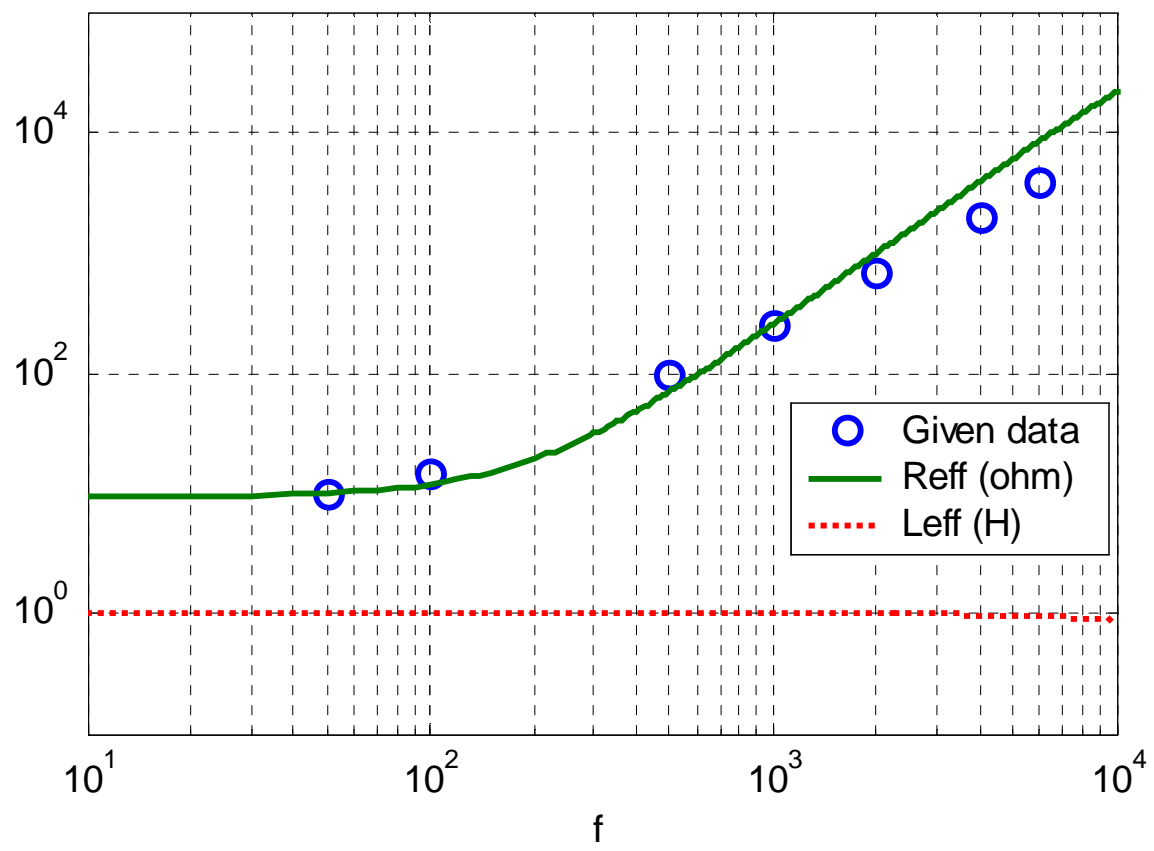

Figure 4.5 Effective R and L by Equation (4.3) with Foster Circuit with One Cell

From least square curve fitting, $\mathrm{R}_{\mathrm{P}}$ and $\mathrm{L}$ (part of the leakage inductance) can be obtained as $R_{P}=15,031 \Omega$ and $L=0.2731 \mathrm{H}$. In Figure 4.6, the Foster circuit with one cell gives more correct $\mathrm{R}(\mathrm{f})$ in the given frequency range. However, The effective $\mathrm{L}$ is not constant in the given frequency range. Therefore, this method is not as robust of a frequency-dependent representation as desired. 


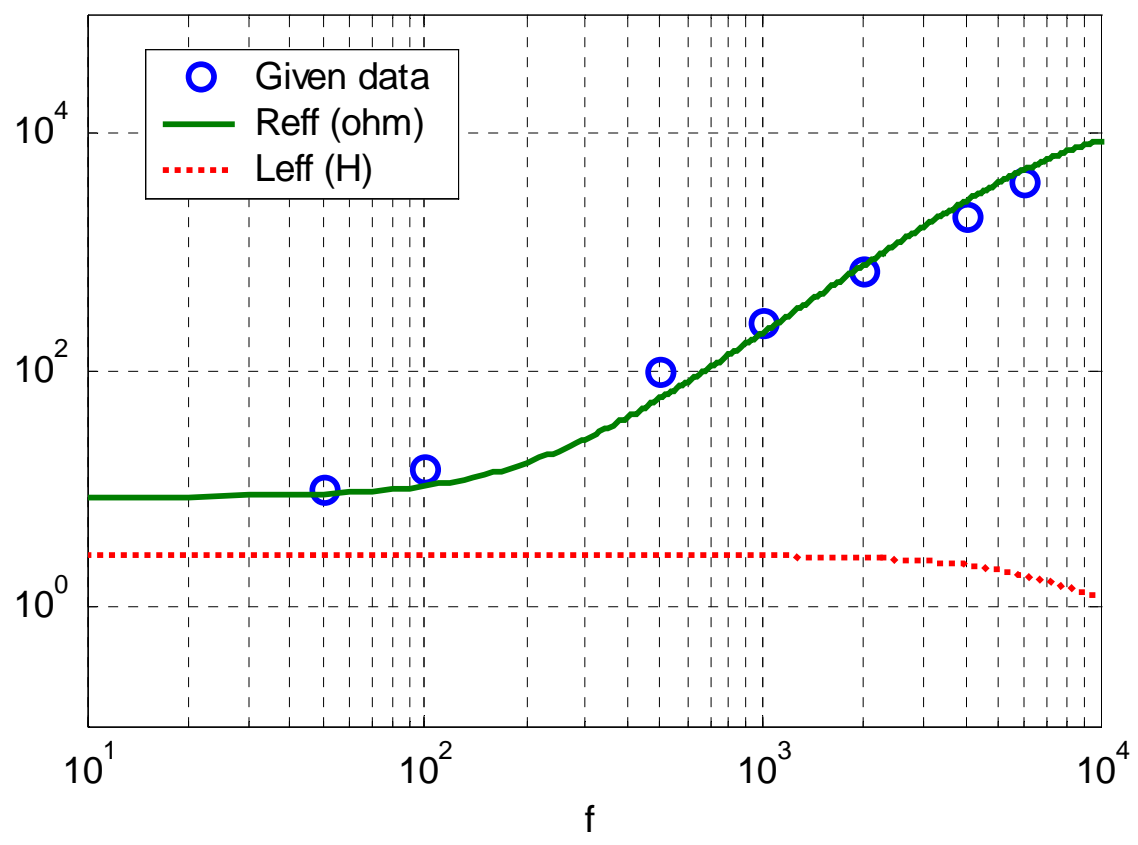

Figure 4.6 Effective R and L by Curve Fitting with Foster Circuit with One Cell

A series Foster circuit with two cells, as in Figure 4.7, is necessary for more accurate representation. From the least square curve fitting using Equations (4.4) and (4.5), $\mathrm{R}_{1}, \mathrm{~L}_{1}, \mathrm{R}_{2}$ and $L_{2}$ can be obtained as $153.7637 \Omega, 0.0424 \mathrm{H}, 104580 \Omega$, and $0.5682 \mathrm{H}$ respectively. Figure 4.8 shows that the effective resistance is well-matched and the equivalent $\mathrm{L}$ is more constant through the given frequency range. The equivalent L in Figure 4.8 should be part of the leakage inductance.

$$
\begin{aligned}
& R_{\text {eff }}=R_{s}+\frac{R_{1} \cdot\left(\omega L_{1}\right)^{2}}{R_{1}^{2}+\left(\omega L_{1}\right)^{2}}+\frac{R_{2} \cdot\left(\omega L_{2}\right)^{2}}{R_{2}^{2}+\left(\omega L_{2}\right)^{2}} \\
& F\left(R_{1}, L_{1}, R_{2}, L_{2}\right)=\sum_{i=1}^{N}\left[\text { Riven }_{i}-R_{\text {eff }}\right]^{2}
\end{aligned}
$$




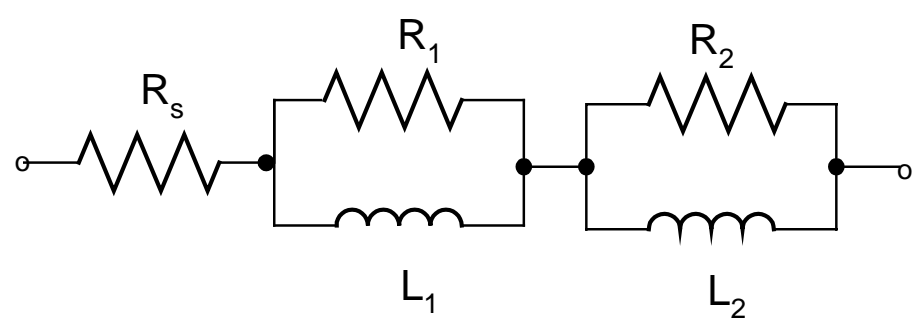

Figure 4.7 Series Foster Circuit with Two Cells

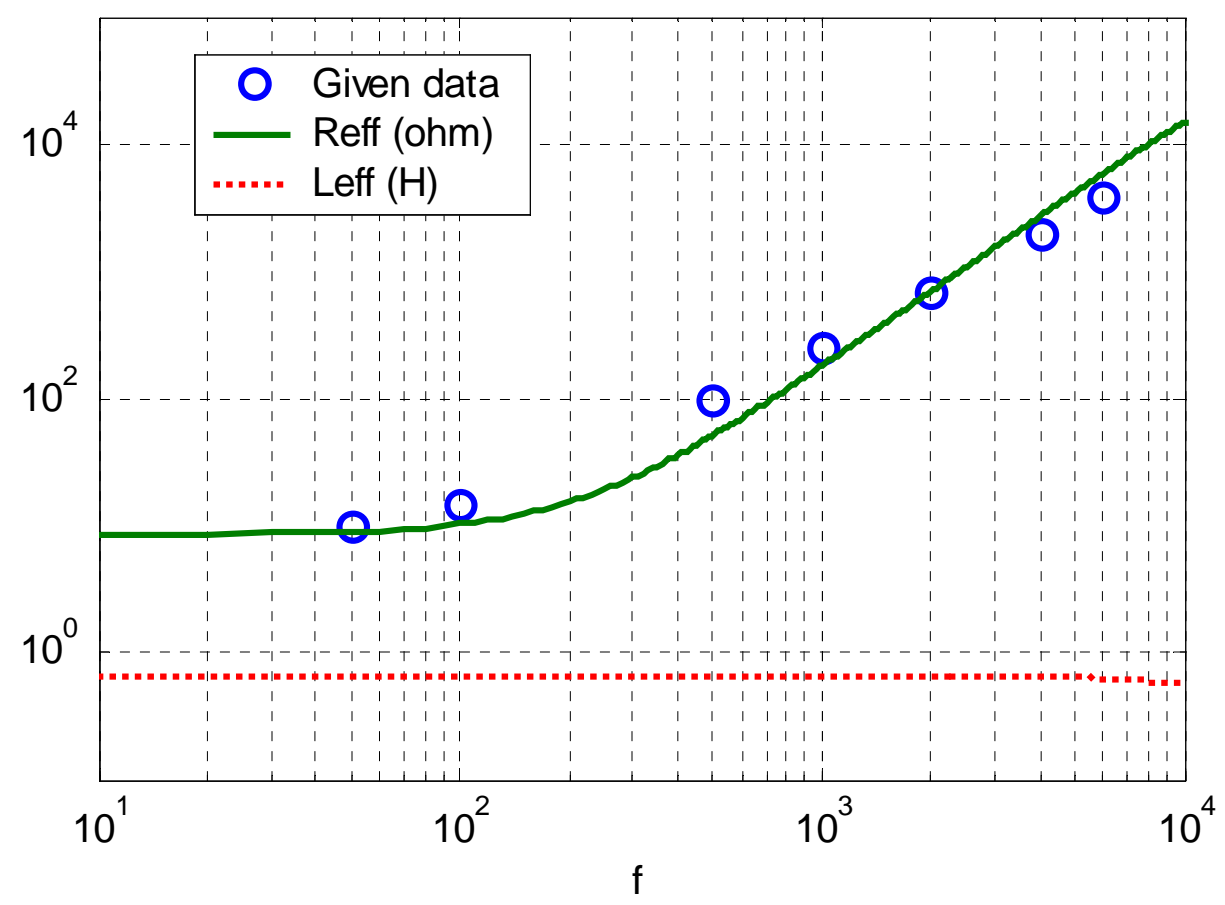

Figure 4.8 Effective R and L by Foster Circuit with Two Cells 


\section{Effective Resistance for Three-Phase Transformer}

From the test report of the example transformer, $\mathrm{R}_{\mathrm{S}, \mathrm{DC}}=0.6766 \Omega, \mathrm{R}_{\mathrm{C}, \mathrm{DC}}=0.1635 \Omega$, turns ratio $=(345-118) / 118=1.9237$, and rated current is 495.35 amps at $345-\mathrm{kV}$ base.

Thus, $\quad \mathrm{R}_{\mathrm{DC}}\left(\mathrm{DC}, \mathrm{H}-\mathrm{L}\right.$ at 3 phase, $\left.75^{\circ} \mathrm{C}\right)=0.6766+1.92372 \times 0.1635=1.282 \Omega$

$\mathrm{R}_{60}(60 \mathrm{~Hz}, \mathrm{H}-\mathrm{L}$ at 3 phase $)=\mathrm{P} / \mathrm{I}^{2}=378.940 \mathrm{~kW} / 495.352=1.54435 \Omega$

Therefore, Ratio of $R_{60} / R_{D C}$ is $1.54435 / 1.282=1.205$.

$R_{H X}, R_{H X}$, and $R_{X Y}$ at $60 \mathrm{~Hz}$ for an autotransformer can be calculated from copper losses. $R_{C}, R_{S}$, and $R_{T}$ at $60 \mathrm{~Hz}$ are calculated using Equation (4.6). However, the resistances of the common winding and the series winding did not match well with the DC resistance value stated on the factory test report, so the validation of the recorded data was questioned. If the current density is assumed to be the same for the two windings, the DC resistance should be correct. Thus $R_{C}, R_{S}, R_{T}$ at $60 \mathrm{~Hz}$ were estimated by 1.25 times DC resistances in order to match coil losses in Table 4.2. The recalculated losses differ from test data, but $\%$ differences are less than $10 \%$.

$$
\begin{aligned}
& R_{S C}=R_{H X} \cdot\left(\frac{N}{N-1}\right)^{2}, \quad R_{C T}=R_{X Y} \\
& R_{T S}=R_{H Y} \cdot\left(\frac{N}{N-1}\right)-R_{X Y} \cdot\left(\frac{1}{N-1}\right)+R_{H X} \cdot\left(\frac{N}{(N-1)^{2}}\right) \\
& R_{T}=\frac{R_{C T}+R_{T S}-R_{S C}}{2}, \quad R_{C}=\frac{R_{C T}+R_{S C}-R_{T S}}{2} \\
& R_{S}=\frac{R_{T S}+R_{S C}-R_{C T}}{2}, \quad N=\frac{V_{H}}{V_{X}}
\end{aligned}
$$


Table 4.2 $\mathrm{R}_{\mathrm{DC}}$ and $\mathrm{R}_{60}$ for Example Transformer

\begin{tabular}{|c|c|c|c|c|c|}
\hline & Winding-T & Winding-C & Winding-S & Loss (W) & \\
\hline Turns(Voltage Ratio) & 13.8 & 68.127 & 131.059 & & \\
\hline $\begin{array}{c}\text { Current and Loss } \\
\text { @ H-X, 296MVA }\end{array}$ & - & 953.1 & 495.35 & 378,940 & \\
\hline $\begin{array}{c}\text { Current and Loss } \\
\text { @ H-Y, 77MVA }\end{array}$ & 1860 & 128.8 & 128.8 & 258,760 & \\
\hline $\begin{array}{c}\text { Current and Loss } \\
\text { @ H-X, 77MVA }\end{array}$ & 1860 & 376.7 & - & 237,680 & \\
\hline & & & & & \\
\hline By R $(\Omega)$ & 0.0175 & 0.0545 & 0.2098 & Loss $(\mathrm{W})$ & $\%$ \\
\hline Loss $(296 \mathrm{MVA}, \mathrm{H}-\mathrm{X})$ & - & 148,523 & 154,437 & 302,960 & 80 \\
\hline Loss (77MVA, H-Y) & 181,629 & 2,712 & 10,441 & 194,783 & 75 \\
\hline Loss (77MVA, X-Y) & 181,629 & 23,201 & - & 204,830 & 86 \\
\hline & & & & & \\
\hline R60 $(\Omega)$ From Loss & 0.0226 & 0.0082 & 0.4847 & Loss (W) & $\%$ \\
\hline Loss (296MVA,H-X) & - & 22,347 & 356,795 & 379,142 & 100 \\
\hline Loss (77MVA, H-Y) & 234,561 & 408 & 24,123 & 259,092 & 100 \\
\hline Loss (77MVA, X-Y) & 234,561 & 3,491 & - & 238,052 & 100 \\
\hline & & & & & \\
\hline & 0.0219 & 0.0681 & 0.2623 & Loss (W) & $\%$ \\
\hline Adjusted R60 $(\Omega)$ & - & 185,654 & 193,046 & 378,700 & 100 \\
\hline Loss (296MVA,H-X) & 227,036 & 3,390 & 13,052 & 243,478 & 94 \\
\hline Loss (77MVA, H-Y) & 227,036 & 29,001 & - & 256,038 & 108 \\
\hline Loss (77MVA, X-Y) & & & & \\
\hline
\end{tabular}

There is no test data for frequency higher than $60 \mathrm{~Hz}$. Using Table 4.1, the effective resistance for the given frequencies can be assumed as in Table 4.3. From least square curve fitting [54], parameters in Figure 4.7 can be obtained as Table 4.4. From Figure 4.9, the effective resistance is well-matched and the effective inductance is constant in the given frequency range. The frequency-dependency of coil resistance for the example transformer is given in Table 4.3. R's and L's for the Foster equivalent circuit are given in Table 4.4. 
Table 4.3 Effective Resistance for Example Transformer

\begin{tabular}{|c|c|c|c|c|c|c|c|c|}
\hline Frequency & $0 \mathrm{~Hz}$ & $\mathbf{6 0 ~ H z}$ & $.1 \mathrm{kHz}$ & $0.5 \mathrm{kHz}$ & $1 \mathrm{kHz}$ & $2 \mathrm{kHz}$ & $4 \mathrm{kHz}$ & $6 \mathrm{kHz}$ \\
\hline $\begin{array}{c}\text { Typical } \\
\text { Ratio }\end{array}$ & 0.8301 & $\mathbf{1 . 0}$ & $\mathbf{1 . 3 6 3 6}$ & $\mathbf{9 . 0 9 0 9}$ & $\mathbf{2 2 . 7 2 7}$ & $\mathbf{6 0 . 9 0 9}$ & $\mathbf{1 8 1 . 8 2}$ & $\mathbf{3 5 4 . 5 4}$ \\
\hline $\mathrm{R}_{\mathrm{HL}}(\Omega)$ & 1.2820 & $\mathbf{1 . 5 4 4 4}$ & 2.1060 & 14.040 & 35.100 & 94.068 & 280.80 & 547.56 \\
\hline $\mathrm{R}_{\mathrm{S}}(\Omega)$ & 0.2098 & $\mathbf{0 . 2 6 2 3}$ & 0.3577 & 2.3845 & 5.9614 & 15.977 & 47.691 & 92.997 \\
\hline $\mathrm{R}_{\mathrm{C}}(\Omega)$ & 0.0545 & $\mathbf{0 . 0 6 8 1}$ & 0.0929 & 0.6191 & 1.5477 & 4.1478 & 12.382 & 24.145 \\
\hline $\mathrm{R}_{\mathrm{T}}(\Omega)$ & 0.0175 & $\mathbf{0 . 0 2 1 9}$ & 0.0299 & 0.1991 & 0.4977 & 1.334 & 3.982 & 7.765 \\
\hline
\end{tabular}

Table 4.4 Parameters for Equivalent Circuit for Example Transformer

\begin{tabular}{|c|c|c|c|c|c|}
\hline & $\mathrm{R}_{\mathrm{S}}(\Omega)$ & $\mathrm{R}_{1}(\Omega)$ & $\mathrm{L}_{1}(\Omega)$ & $\mathrm{R}_{2}(\Omega)$ & $\mathrm{L}_{2}(\Omega)$ \\
\hline $\mathrm{R}_{\mathrm{HL}}$ & 1.2820 & 24.8081 & 2.2620 & 40,364 & 49.0477 \\
\hline $\mathrm{R}_{\mathrm{S}}$ (series) & 0.2098 & 3.8782 & 0.3841 & 11,202 & 10.7159 \\
\hline $\mathrm{R}_{\mathrm{C}}$ (common) & 0.0545 & 0.9874 & 0.0993 & 11,439 & 5.5231 \\
\hline $\mathrm{R}_{\mathrm{T}}$ (tertiary) & 0.0175 & 0.3158 & 0.0319 & 11,581 & 3.1521 \\
\hline
\end{tabular}

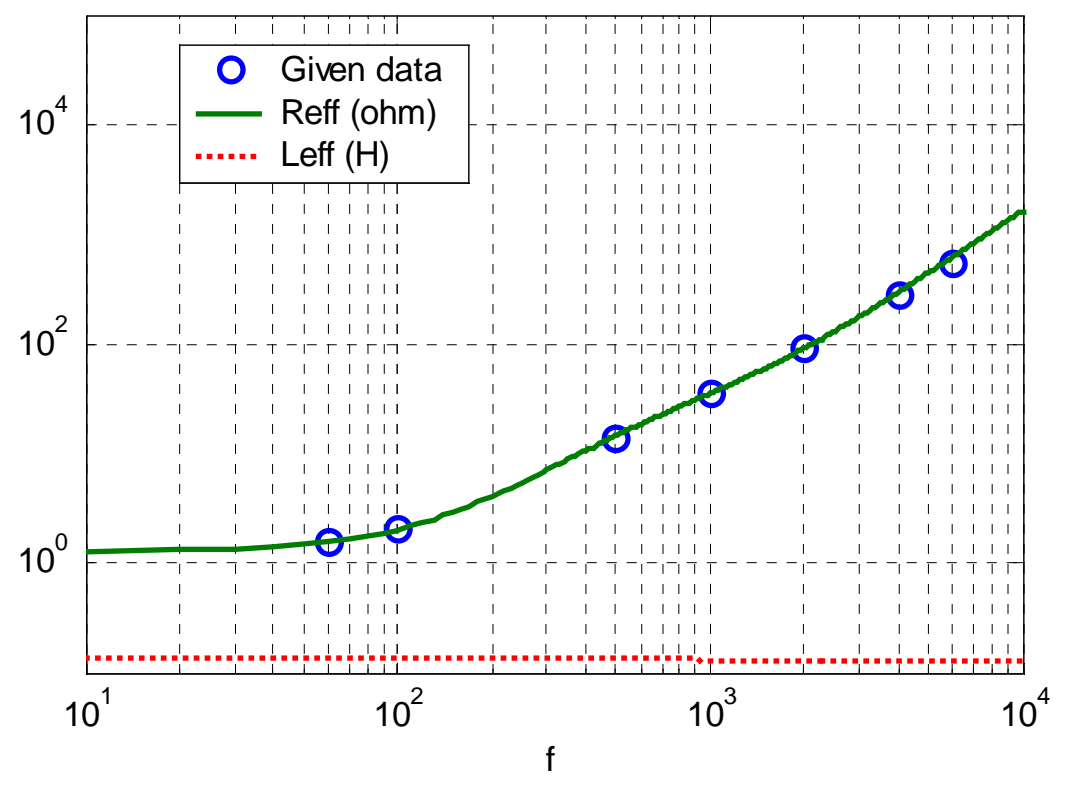


Figure 4.9 Effective $\mathrm{R}_{\mathrm{HL}}$ and $\mathrm{L}_{\mathrm{HL}}$ for Example Transformer

by Foster Circuit with Two Cells

\subsection{Winding Capacitance}

Capacitance considerations were introduced in Section 2.5. Some test reports give the capacitance values shown in Figure 4.10. However, most test reports do not give these capacitance values.

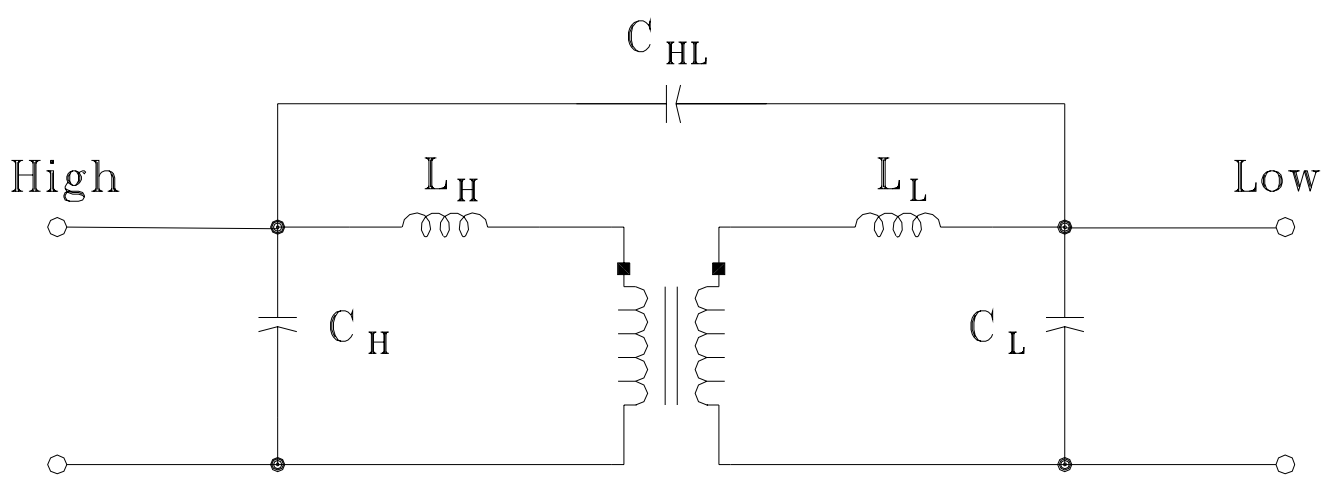

Figure 4.10 Equivalent Circuit for Capacitance

If design information is given, the calculation of various capacitances is possible. In a transformer, the inner and outer sides of the windings are like parallel plates of a capacitor with oil and paper as the dielectric. The equation for parallel plate capacitance is generally valid for calculating the various capacitances.

$$
C=\frac{A \cdot \varepsilon_{0} \cdot \varepsilon_{r}}{d} \quad(\text { Farads })
$$

where,

$A=$ Area of one of the plates forming the capacitance in $\mathrm{m}^{2}$

$d=$ Distance between the two parallel plates in $m$

$\varepsilon_{o}=$ permittivity of free space

$\varepsilon_{r}=$ relative permittivity of the dielectric. For oil impregnated paper, typically 4.2 
Substituting the values of winding surface areas and gaps between windings in Equation (4.7), the winding capacitance can be calculated. For core-type transformers, the winding capacitances can in this way be approximated by parallel plate capacitance formulas in which the capacitance is proportional to the area of the plates and inversely proportional to the separation between the plates. The size of the plates can be approximated as being proportional to the square root of the MVA, while their separation can be approximated as being proportional to the BIL level for the higher of the two windings involved. For a twowinding transformer, the capacitance of the HV winding to ground is generally less than the capacitance of the LV winding to ground because of the increased clearance needed for the HV winding.

For a shell-type transformer, the parallel plate model for the transformer winding to ground capacitance calculations is not as accurate or as applicable. For the HV to LV capacitance, the parallel plate representation is quite reasonable and accurate. The HV to LV capacitance is proportional to the number of HV to LV gaps. The presence of a tertiary winding can affect the capacitances considerably [24].

However, the calculation of winding capacitance is not possible in cases where the detailed design information is not available. Instead the effective terminal capacitance can be determined based on the frequency of oscillation of each winding by using Equations (2.10) through (2.13).

If TRV frequency values are known, effective capacitance values can be calculated by Equation (4.8) using the apparent TRV frequency values and transformer leakage reactance.

From reference [26], 


$$
C_{e f f}=\frac{1}{\left(2 \pi f_{T R V}\right)^{2} \cdot L}=\frac{k}{X_{L} \cdot f_{T R V}{ }^{2}}=\frac{k}{\frac{k V^{2}}{M V A} \cdot X \cdot f_{T R V}{ }^{2}}
$$

where, $k=$ constant related to system frequency $(k=9.55$ at $60 \mathrm{~Hz})$

$f_{T R V}=$ apparent $T R V$ frequency $(\mathrm{kHz})$

$L=$ transformer leakage inductance (henries)

$X_{L}=$ transformer leakage reactance (ohms)

$C_{\text {eff }}=$ effective capacitance $(\mu F)$,

$K V=$ Line to Line Voltage $(k V)$

$M V A=$ Transformer rating (MVA),

$X=$ reactance based on $M V A(p u)$

TRV frequency is inversely proportional to the square root of the nominal voltage and proportional to the square root of the fault current. It also tends to decrease as MVA size increases, since capacitance apparently is a function of transformer construction including physical size related to the MVA size. Figure B.2 of ANSI/IEEE C37.011-1994 [1] shows well that TRV frequency decreases as MVA size increases. Thus, TRV frequency is:

$$
f_{\text {TRV }}=\frac{\sqrt{\text { fault current }}}{\sqrt{\text { voltage }} \cdot f(\text { MVA size })}
$$

The capacitive coupling ratio was defined as $C_{H L} /\left(C_{H L}+C_{L}\right)$ in Section 2.5 .

From the effective capacitance at the high-voltage side, the effective capacitance at the low-voltage side and the capacitive coupling ratio, the capacitance for each winding and coupling are:

$$
\begin{aligned}
& C_{H L}=\text { Capacitive coupling ratio } \times C_{\text {eff }} \text { at the low-voltage winding } \\
& C_{L}=C_{\text {eff }} \text { at the low-voltage winding }-C_{H L} \\
& C_{H}=C_{\text {eff }} \text { at the high-voltage winding }-C_{H L}
\end{aligned}
$$




\section{Capacitive Coupling for Three-Phase Transformer}

Capacitive effects may be significant and need to be included in the model. The major coupling capacitances between transformer core and between windings are shown in Figures 4.11 and 4.12. Three such capacitances $\left(\mathrm{C}_{\mathrm{Sg}}, \mathrm{C}_{\mathrm{Cg}}, \mathrm{C}_{\mathrm{Tg}}\right)$ for each phase need to be added. The windings are separated by insulating material (oil and paper) forming parallel plate capacitances. There are two such capacitances in the transformer $\left(\mathrm{C}_{\mathrm{CT}}\right.$ and $\left.\mathrm{C}_{\mathrm{ST}}\right)$. These capacitances are connected from the outside of the tertiary or common windings to the insides of the common or series windings. Also, two adjacent high-voltage windings are separated by insulation forming a capacitance $\left(\mathrm{C}_{\mathrm{SS}}\right)$. These couple the outer side of one winding to the outer side of the other. The capacitance between $\mathrm{H}_{1}$ and $\mathrm{H}_{3}$ is negligible due to the large distance between the two and the presence of winding $\mathrm{H}_{2}$. After addition of these capacitances in Figures 4.11 and 4.12, the result is shown in Figure 4.13.

The effective capacitances for the example transformer are shown in Table 4.5.

Table 4.5 Effective Capacitances for the Example Transformer

\begin{tabular}{|c|c|c|c|c|c|c|}
\hline $\begin{array}{c}\text { Source } \\
\text { Side }\end{array}$ & $\begin{array}{c}\text { Fault } \\
\text { Side }\end{array}$ & $\begin{array}{c}\text { Rating } \\
\text { (MVA) }\end{array}$ & $\mathrm{Z}(\%)$ & $\begin{array}{c}\text { Fault } \\
\text { Current } \\
(\mathrm{kA})\end{array}$ & $\begin{array}{c}\text { TRV } \\
\text { Frequency } \\
(\mathrm{kHz}) \\
\text { from Figure } \\
\text { B.2 of [1] }\end{array}$ & $\begin{array}{c}\text { Effective } \\
\text { Capacitance } \\
\text { (pF) From } \\
\text { Equation } \\
(4.8)\end{array}$ \\
\hline $345 \mathrm{kV}$ & $118 \mathrm{kV}$ & 296 & 6.21 & 8.0 & 8.5 & 5,293 \\
\hline $118 \mathrm{kV}$ & $345 \mathrm{kV}$ & 296 & 6.21 & 23.3 & 18.0 & 10,090 \\
\hline $345 \mathrm{kV}$ & $13.8 \mathrm{kV}$ & 296 & 55.9 & 0.9 & 3.8 & 2,942 \\
\hline $13.8 \mathrm{kV}$ & $345 \mathrm{kV}$ & 296 & 55.9 & 22.2 & 68.0 & 5,743 \\
\hline $118 \mathrm{kV}$ & $13.8 \mathrm{kV}$ & 296 & 42.1 & 3.4 & 12.0 & 3,349 \\
\hline $13.8 \mathrm{kV}$ & $118 \mathrm{kV}$ & 296 & 42.1 & 29.4 & 72.0 & 6,801 \\
\hline
\end{tabular}




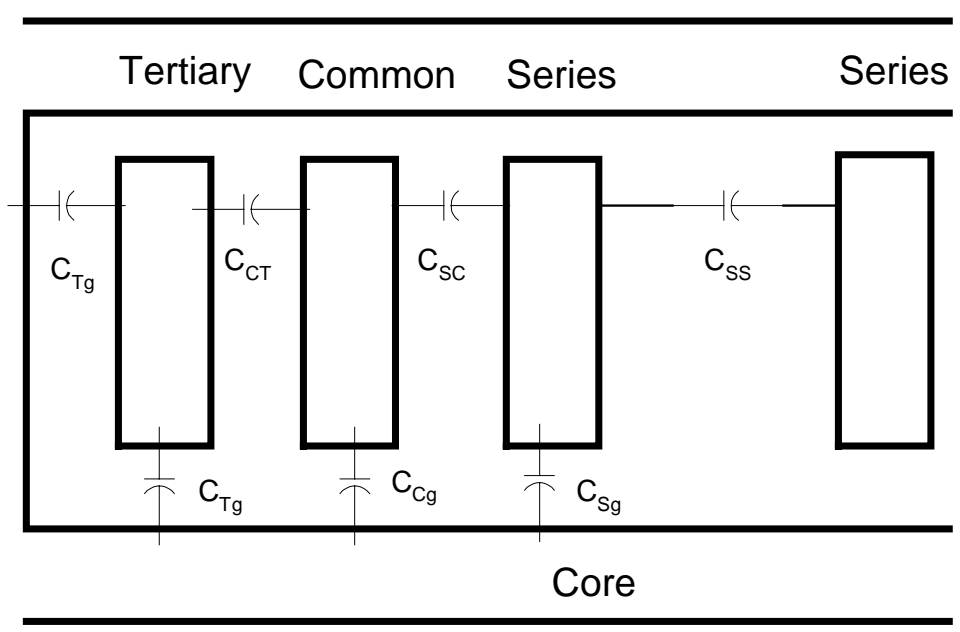

Figure 4.11 Capacitances of Concentric Winding

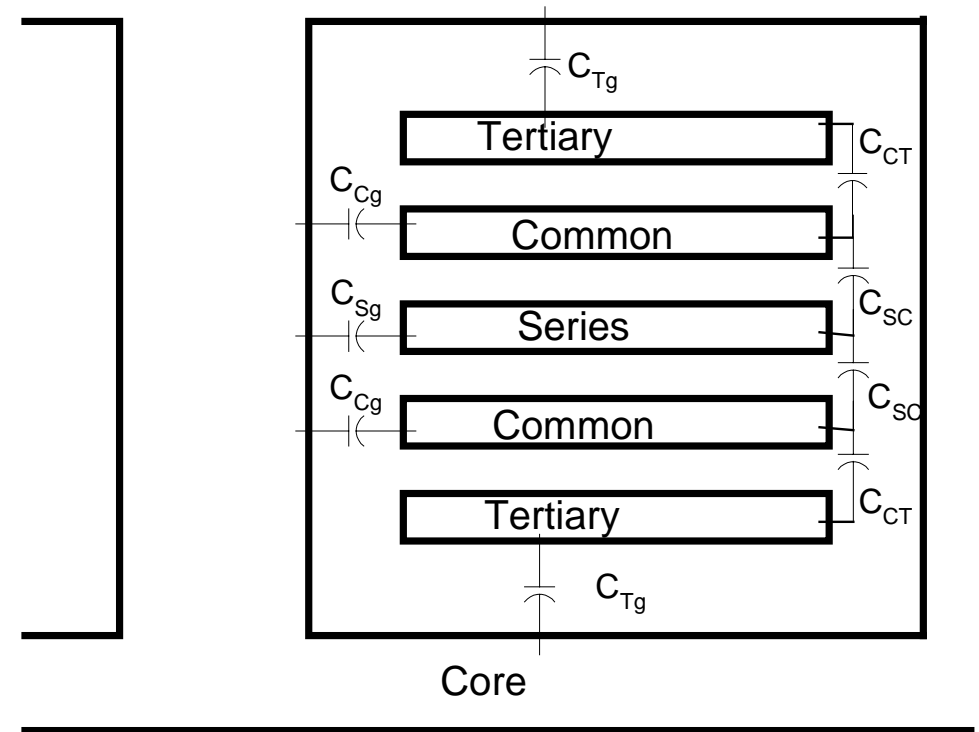

Figure 4.12 Capacitances of Pancake Winding 


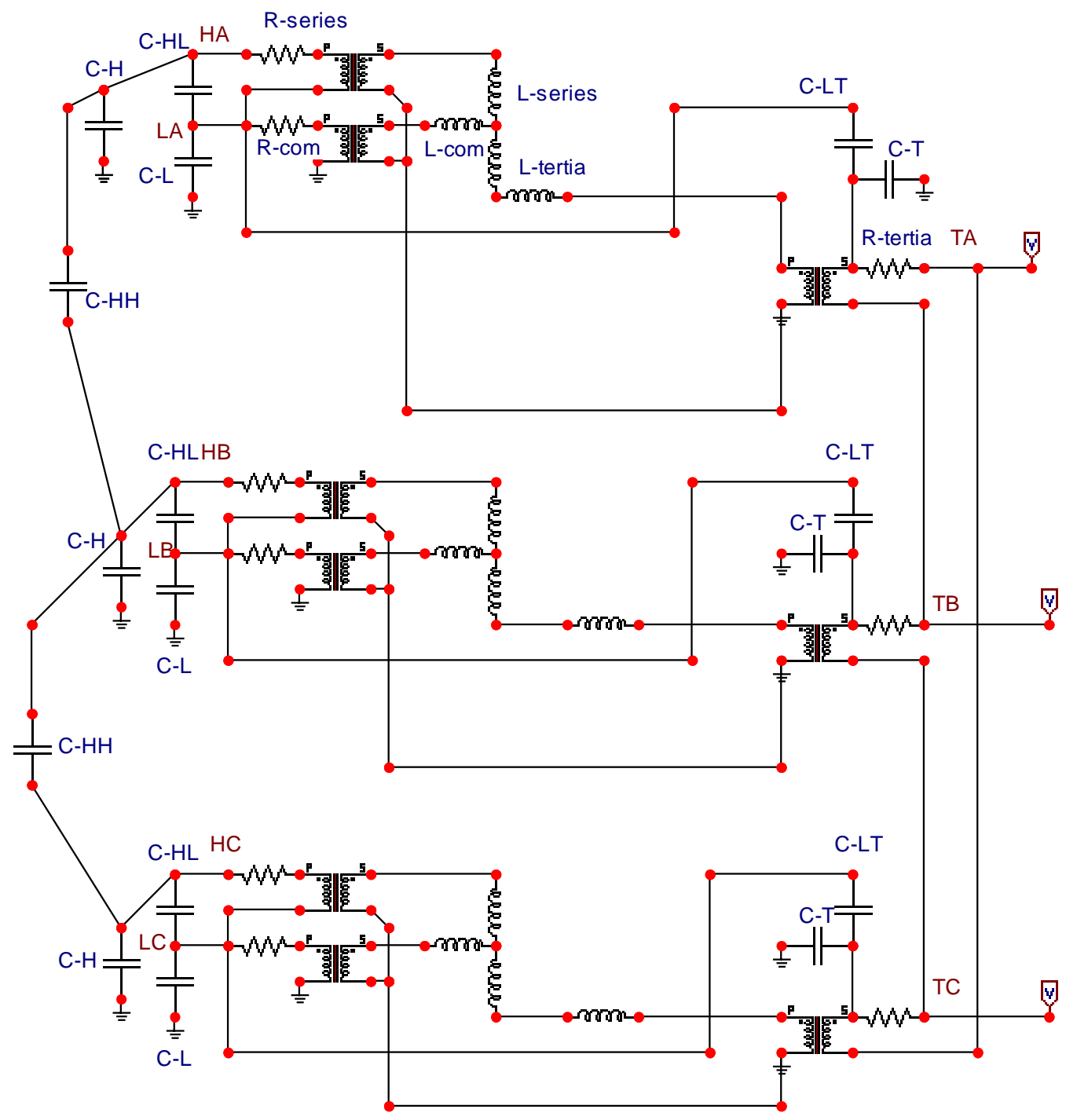

Figure 4.13 Capacitances for Three-winding Three-phase Autotransformer 


\section{High voltage $(345 \mathrm{kV})$ to Low Voltage $(118 \mathrm{kV})$}

The capacitive coupling ratio is generally lower than 0.4 [50]. If the capacitive coupling ratio for low voltage $(118 \mathrm{kV})$ winding is assumed as $0.3, C_{H L}=3,027 \mathrm{pF}, C_{L}=7,063 \mathrm{pF}$, $C_{H}=2,266 \mathrm{pF}$ are obtained from Equations (4.9) through (4.11)

\section{High voltage $(345-\mathrm{kV})$ to Tertiary voltage $(13.8 \mathrm{kV})$}

Lower voltage windings have larger capacitances [24]. Here, the capacitive coupling ratios for lower voltage windings are assumed smaller than those of higher voltage windings. If the capacitive coupling ratio for the tertiary voltage $(13.8 \mathrm{kV})$ winding is assumed as $0.1, C_{H T}=$ $574 \mathrm{pF}, C_{T}=10,336 \mathrm{pF}, C_{H}=2,368 \mathrm{pF}$ are obtained from Equations (4.9) through (4.11). The tertiary-voltage windings are delta-connected. Therefore, for the tertiary-voltage windings, two times the value from Equation (4.10), 5,168 pF, was assumed. This is explained in Section 13.2.2 of [24].

\section{Low voltage $(118-\mathrm{kV})$ to Tertiary voltage $(13.8 \mathrm{kV})$}

If the capacitive coupling ratio for the tertiary voltage $(13.8 \mathrm{kV})$ winding is assumed as 0.1 , $C_{L T}=680 \mathrm{pF}, C_{T}=12,242 \mathrm{pF}, C_{L}=2,669 \mathrm{pF}$ are obtained from Equations (4.9) through (4.11). The tertiary-voltage windings are delta-connected. Therefore, for the tertiary-voltage windings, two times the value from Equation (4.10), 6,121 pF, again, based on Section 13.2.2 of [24].

Two capacitance values were calculated for the $\mathrm{C}_{\mathrm{H}}, \mathrm{C}_{\mathrm{L}}$ and $\mathrm{C}_{\mathrm{T}}$. In this work, the capacitance values calculated from the higher source voltage were chosen. The selected winding capacitances for the example transformer are shown in Table 4.6. 
Table 4.6 Selected Winding Capacitances for the Example Transformer

\begin{tabular}{|c|c|c|c|c|c|}
\hline$C_{H L}$ & $C_{L}$ & $C_{H}$ & $C_{H T}$ & $C_{T}$ & $C_{L T}$ \\
\hline $3,027 p F$ & $7,063 p F$ & $2,266 p F$ & $574 p F$ & $10,336 p F$ & $681 p F$ \\
\hline
\end{tabular}

The effective capacitances are in the range of Table B.9 of [1] (see Table 4.7) and the capacitance for each winding is also in the range of Figure 13.8 of [24].

Table 4.7 Typical Effective Capacitance Range from Table B.9 of [1]

\begin{tabular}{|c|c|c|}
\hline Transformer Size (MVA) & Voltage $(\mathrm{kV})$ & Effective Capacitance $(\mathrm{pF})$ \\
\hline $1 \sim 10$ & $15 \mathrm{kV} \sim 121 \mathrm{kV}$ & $900 \sim 10,000$ \\
\hline \multirow{2}{*}{$10 \sim 100$} & $15 \mathrm{kV} \sim 121 \mathrm{kV}$ & $2000 \sim 12,000$ \\
\cline { 2 - 3 } & $121 \mathrm{kV} \sim 550 \mathrm{kV}$ & $2000 \sim 6500$ \\
\hline $100 \sim 1000$ & $121 \mathrm{kV} \sim 550 \mathrm{kV}$ & $3500 \sim 16,000$ \\
\hline
\end{tabular}

Couplings between windings in the same phase are considered. There are possible couplings between $\mathrm{HV}$ of phase- $\mathrm{A}$ and $\mathrm{HV}$ of phase-B and between $\mathrm{HV}$ of phase- $\mathrm{B}$ and $\mathrm{HV}$ of phase-C. These capacitances are assumed smaller than $\mathrm{C}_{\mathrm{HL}}$, since the insulation thickness should be bigger. In this work, it is assumed as one third of $\mathrm{C}_{\mathrm{HL}}$. When the example transformer is energized with rated voltage, the capacitance currents can be calculated as given in Table 4.8 .

When the example transformer is energized at rated voltage from the tertiary $(13.8 \mathrm{kV})$ with no load, the resulting open-circuit test currents are presented in Table 4.9. The magnetizing current is about $60.63 \mathrm{~A}$ from Table 4.9. However, the apparent magnetizing current is about $53.86 \mathrm{~A}$, if the winding capacitance is neglected. The difference is about $11 \%$. Therefore, the magnetizing circuit parameter may have a large percentage error if the winding capacitance is neglected, especially at and below the knee of the magnetization curve. 
Table 4.8 Capacitance Current for Example Transformer

\begin{tabular}{|c|c|c|c|c|c|}
\hline & $\mathrm{C}(\mathrm{nF})$ & $\begin{array}{c}\text { Voltage } \\
(\mathrm{kV})\end{array}$ & $\mathrm{I}_{\mathrm{C}}(\mathrm{A})$ & $\begin{array}{c}\mathrm{I}_{\mathrm{C}}(\mathrm{A}) \\
@ \text { 13.8-kV } \\
\text { Winding }\end{array}$ & $\begin{array}{c}\mathrm{I}_{\mathrm{C}}(\mathrm{A}) \\
\text { 13.8-kV } \\
\text { Line }\end{array}$ \\
\hline Tertiary-g & 10.3 & 7.967 & 0.031 & 0.031 & 0.054 \\
\hline Common-g & 7.0 & 68.127 & 0.180 & 0.888 & 1.537 \\
\hline Series-g & 2.3 & 199.186 & 0.173 & 2.493 & 4.318 \\
\hline C-T & 0.7 & 60.160 & 0.015 & 0.076 & 0.132 \\
\hline H-L & 3.0 & 131.059 & 0.148 & 1.408 & 2.438 \\
\hline H-H & 1.0 & 345.000 & 0.130 & 1.877 & 3.252 \\
\hline Total & & & & 6.773 & 11.730 \\
\hline
\end{tabular}

Table 4.9 Breakdown of Open Circuit Current for Example Transformer

\begin{tabular}{|c|c|c|c|}
\hline$\underset{I_{C A P}}{\downarrow} \overbrace{}^{I_{M}} \nearrow^{I_{E X}}$ & Description & $100 \%$ Voltage & $110 \%$ Voltage \\
\hline $\mathrm{I}_{\mathrm{EX}}(\%)$ & No load current $(\%)$ & 0.76 & 1.71 \\
\hline $\mathrm{I}_{\mathrm{EX}}($ Arms $)$ & No load current (Arms) & 54.338 & 122.261 \\
\hline $\mathrm{V}_{\mathrm{OC}}(\mathrm{Vrms})$ & Open Circuit Test Voltage & 13800 & 15180 \\
\hline $\mathrm{P}_{\mathrm{OC}}(\mathrm{W})$ & Core loss & 297600 & 402240 \\
\hline $\mathrm{I}_{\mathrm{C}}(\mathrm{Arms})$ & Core loss current & 7.188 & 8.833 \\
\hline I_reactive (Arms) & $\begin{array}{c}\text { Reactive component } \\
\text { of no-load current }\end{array}$ & 53.861 & 121.941 \\
\hline I_capacitive (Arms) & $\begin{array}{c}\text { Capacitive current } \\
\text { by winding capacitance }\end{array}$ & 6.773 & 7.450 \\
\hline $\mathrm{I}_{\mathrm{M}}(\mathrm{Arms})$ & $\begin{array}{l}\text { Magnetizing Current } \\
\text { at Winding }\end{array}$ & 60.633 & 129.391 \\
\hline $\mathrm{I}_{\mathrm{M}}($ Arms $)$ at Line & Magnetizing Current at Line & 105.020 & 224.112 \\
\hline
\end{tabular}




\subsection{Magnetic Core Saturation}

One of the traditionally used representations for the core saturation curve is the Frolich equation, Equation (4.12). This equation gives a smooth single-valued anhysteretic curve relating the flux density $\mathrm{B}$ to the magnetizing force $\mathrm{H}$. Only two data points on the curve are needed to fit this equation [51].

$$
B=\frac{H}{a+b \cdot|H|}
$$

Other equations can be applied, but those involve too many variables and it is not possible to fit using typical transformer test reports where only two points on the magnetization curve are given.

Returning our attention to the Frolich equation (4.12),

$$
\begin{gathered}
H=\frac{a \cdot B}{1-|B| \cdot b} \text { and } \mu=\frac{B}{H}=\frac{(1-b \cdot|B|)}{a} \\
\text { where } a=1 / \mu=1 /\left(\mu_{i} \cdot \mu_{o}\right) \text { and } b=\frac{1-\frac{1}{\sqrt{\mu_{i}}}}{B s a t} \\
\mu_{i}: \text { initial relative permeability }(15,000 \sim 50000) \\
\mu_{o}: \text { free space permeability }\left(4 \pi \times 10^{-7}\right)
\end{gathered}
$$

Saturation data for Armco M4 Steel is given in Table 4.10. For example, if two points, $\mathrm{H}=[14.4,55]$ and $\mathrm{B}=[1.2,1.6]$, are chosen, then a fit of "a" $=4.0640$ and " $\mathrm{b} "=0.5511$ for Equations (4.12) and (4.13) can be obtained. Comparatively, if all data points are used, then "a"=4.2776 and " $b "=0.5435$, using the optimization technique of least square curve fitting. Figure 4.14 shows the B-H curves obtained from above methods and it matches well with the given saturation data. 
Table 4.10 Magnetic Saturation Data for Armco M4 Steel

\begin{tabular}{|c|c|c|c|c|c|c|c|c|c|c|c|}
\hline B (T) & 0 & 0.2 & 0.4 & 0.6 & 0.8 & 1.0 & $\mathbf{1 . 2}$ & 1.4 & $\mathbf{1 . 6}$ & 1.7 & 1.8 \\
\hline H (A/m) & 0 & 2 & 4 & 6 & 8.4 & 11.1 & $\mathbf{1 4 . 4}$ & 23 & $\mathbf{5 5}$ & 130 & 416 \\
\hline
\end{tabular}

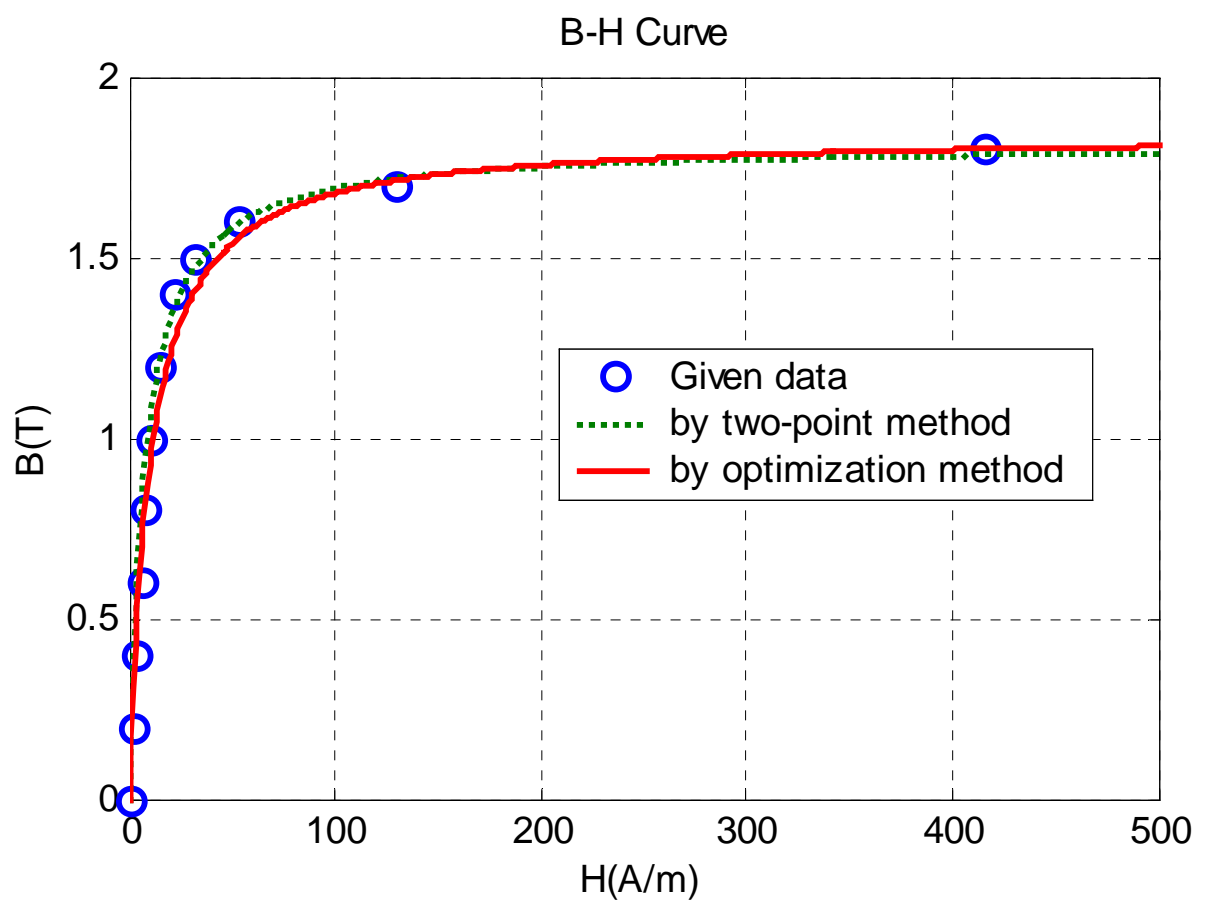

Figure 4.14 Examples of Saturation Curve Fitting using Frolich Equation

If the dimension data is not available and the $\mathrm{B}-\mathrm{H}$ saturation curve of core is given, it is necessary to define scale factors " $\mathrm{x}$ " and " $\mathrm{y}$ " to match the two given points of $\lambda-i$ data with the known B-H saturation curve. In this case, " $\mathrm{x}$ " is for the $\mathrm{i}$-scale and " $\mathrm{y}$ " is for the $\lambda$ scale.

$$
B=\frac{H}{a+b \cdot H} \Rightarrow \lambda / y=\frac{i / x}{a+b \cdot i / x}
$$

Thus,

$$
\lambda=\frac{i \cdot y}{a \cdot x+b \cdot i}=\frac{i}{a \cdot x / y+b \cdot i / y}=\frac{i}{a \cdot \frac{L}{A N}+b \cdot \frac{i}{A N}}
$$

where A= Cross-Sectional Area of core, $L=$ Length of core, $N=$ Turns of Coil 
If two points $\left(\lambda_{1}, i_{1}\right)$ and $\left(\lambda_{2}, i_{2}\right)$ are given, "x" and " $\mathrm{y}$ " are:

$$
\begin{aligned}
& y=\frac{\lambda_{2} \cdot i_{1} \cdot \lambda_{1} \cdot b-\lambda_{1} \cdot i_{2} \cdot \lambda_{2} \cdot b}{\lambda_{2} \cdot i_{1} \cdot \lambda_{1} \cdot i_{2}} \\
& x=\frac{\lambda_{1} \cdot y-\lambda_{1} \cdot i_{1} \cdot b}{a \cdot i_{1}}
\end{aligned}
$$

For example, if the B-H data in Figure 4.14 and the two points, $\mathrm{i}$ (peak-Amp) $=[76.17$ 278.90] and $\lambda(\mathrm{Wb}-\mathrm{t})=[51.76$ 56.94], are given, the two scale factors, $\mathrm{x}=1.3842$ and $y=32.1614$, can be obtained from Equations (4.15) through (4.17). The obtained $\lambda$-i curve is given in Figure 4.15. " $x$ ” and " $y$ " mean $L$ and $A \cdot N$ from Equation (4.17), since $\lambda=B \cdot A \cdot N$ and $i=H \cdot L$.

If both the B-H curve and the dimension data are unknown, $\mathrm{a}=0.1842, \mathrm{~b}=0.0169$ for $\lambda$ i curve are directly obtained from two data points using Equation (4.12). The obtained curve in Figure 4.16 is the same as in Figure 4.15.

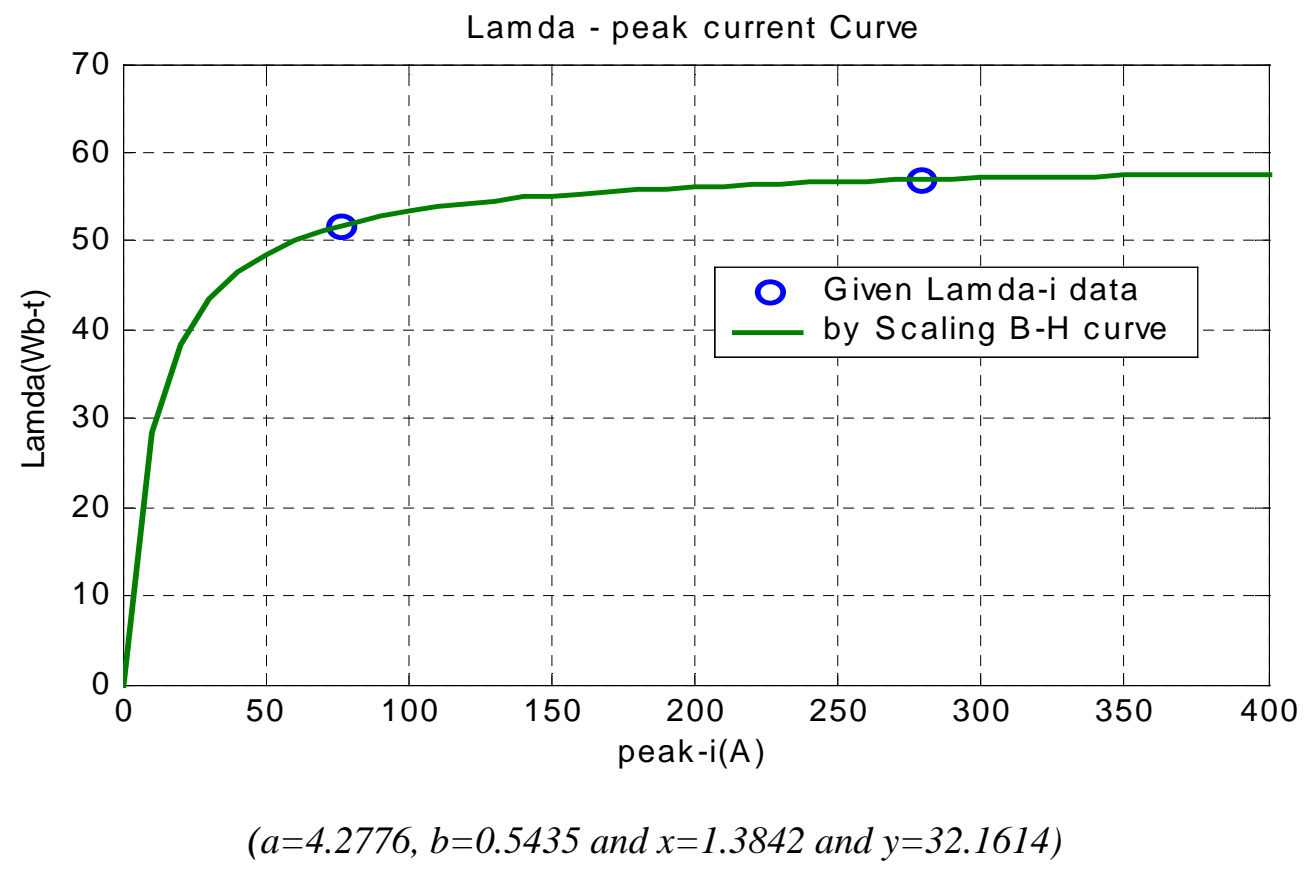

Figure 4.15 Derived Saturation Curve 


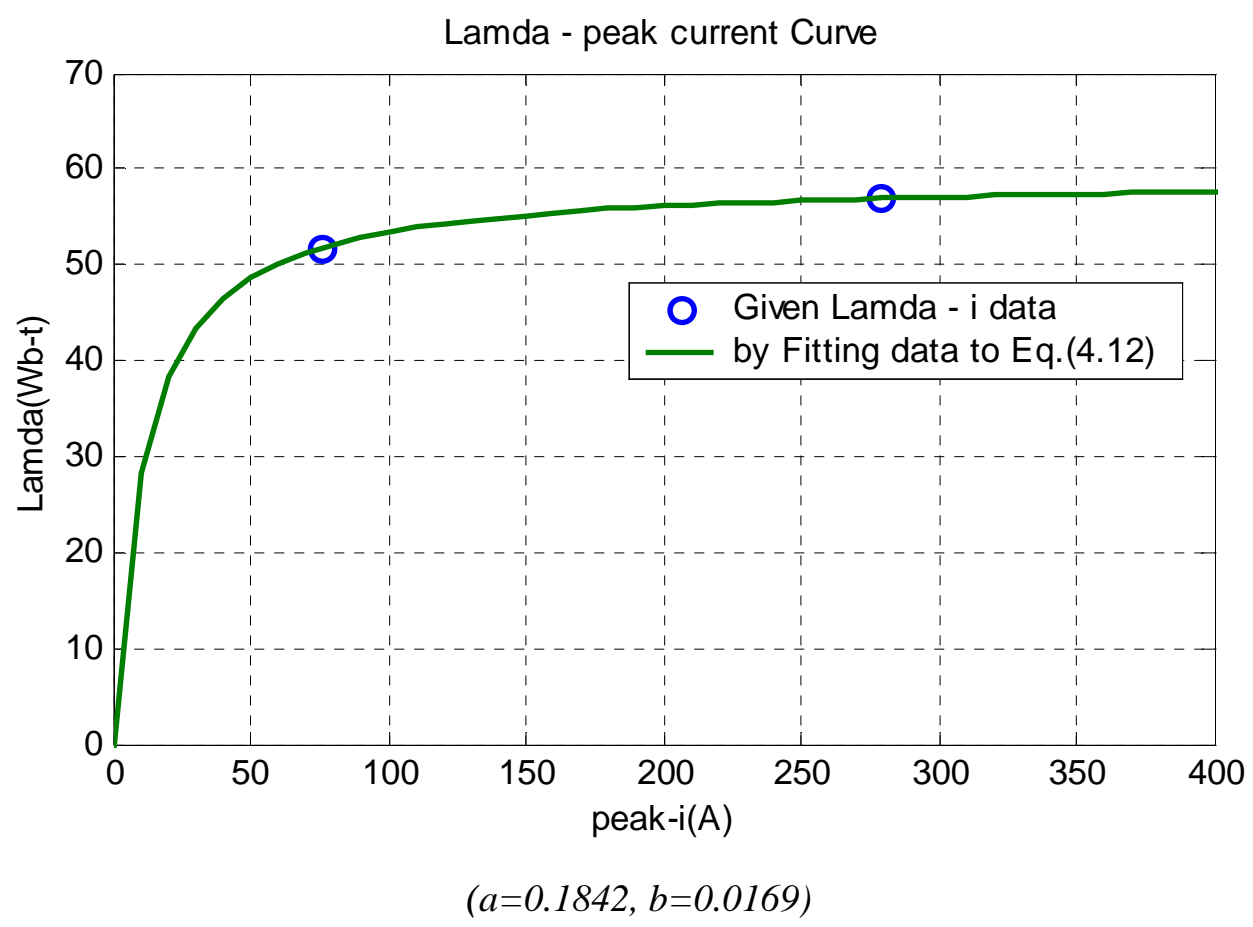

Figure 4.16 Derived Saturation Curve

\subsection{Nonlinear Core Loss}

For describing total average core loss of each section, some characteristic function can be fit to match. Core loss is nonlinear and frequency dependent, and is best considered in that context. However, average power for steady-state excitation at a given sinusoidal frequency can be useful information. The Frolich equation may also be used to fit the average power characteristic:

$$
P_{C}=\frac{a \cdot B}{1-B \cdot b}
$$

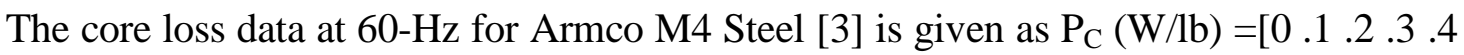

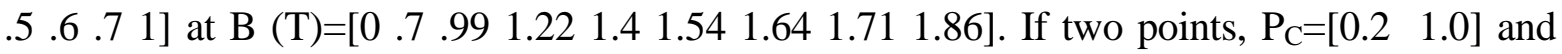
$\mathrm{B}=[.99$ 1.86] are chosen, then "a" $=0.1181$ and $" \mathrm{~b} "=0.4195$ from Equation (4.18). Figure 
4.17 shows that the curve obtained using the equation matches well with the known nonlinear characteristic of the core. Thus, if core dimensions and flux are known for each section, the average core loss for each section can be calculated by Equation (4.19). For a core loss model represented by a separate resistance in parallel with the nonlinear inductance, I-peak and V-peak can be calculated from Irms-Vrms using the SATURATION subroutine of EMTP.

$$
\begin{aligned}
& P_{C}(n)=\frac{a \cdot B}{1-B \cdot b} \cdot A(n) \cdot L(n) \\
& I_{R M S}(n)=\frac{P_{C}(n)}{V_{R M S}(n)}
\end{aligned}
$$

Average DC hysteresis loss data for Armco M4 Steel [3] is given as $P_{H}\left(J / m^{3}\right)=[17.54$ $30.0344 .7073 .21]]$ at $\mathrm{B}(\mathrm{T})=\left[\begin{array}{llll}1 & 1.3 & 1.5 & 1.7\end{array}\right]$. From Equation (4.18), "a" = 9.2071, "b" = 0.4623 can be obtained using all points with least square curve fitting technique. Figure 4.18 shows that the fitted curve using this equation matches well with the known nonlinear characteristic of core. 


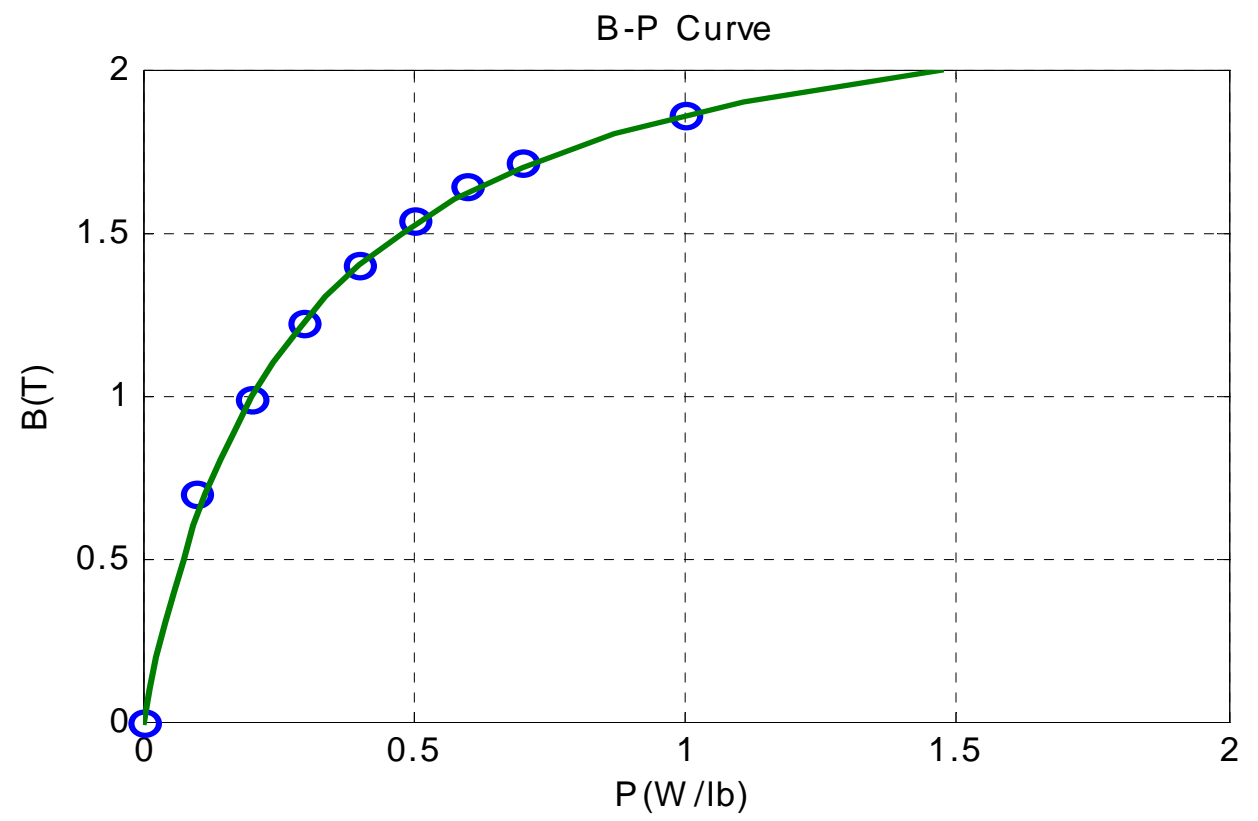

Figure 4.17 Examples of 60-Hz Core Loss Curve using Frolich Equation

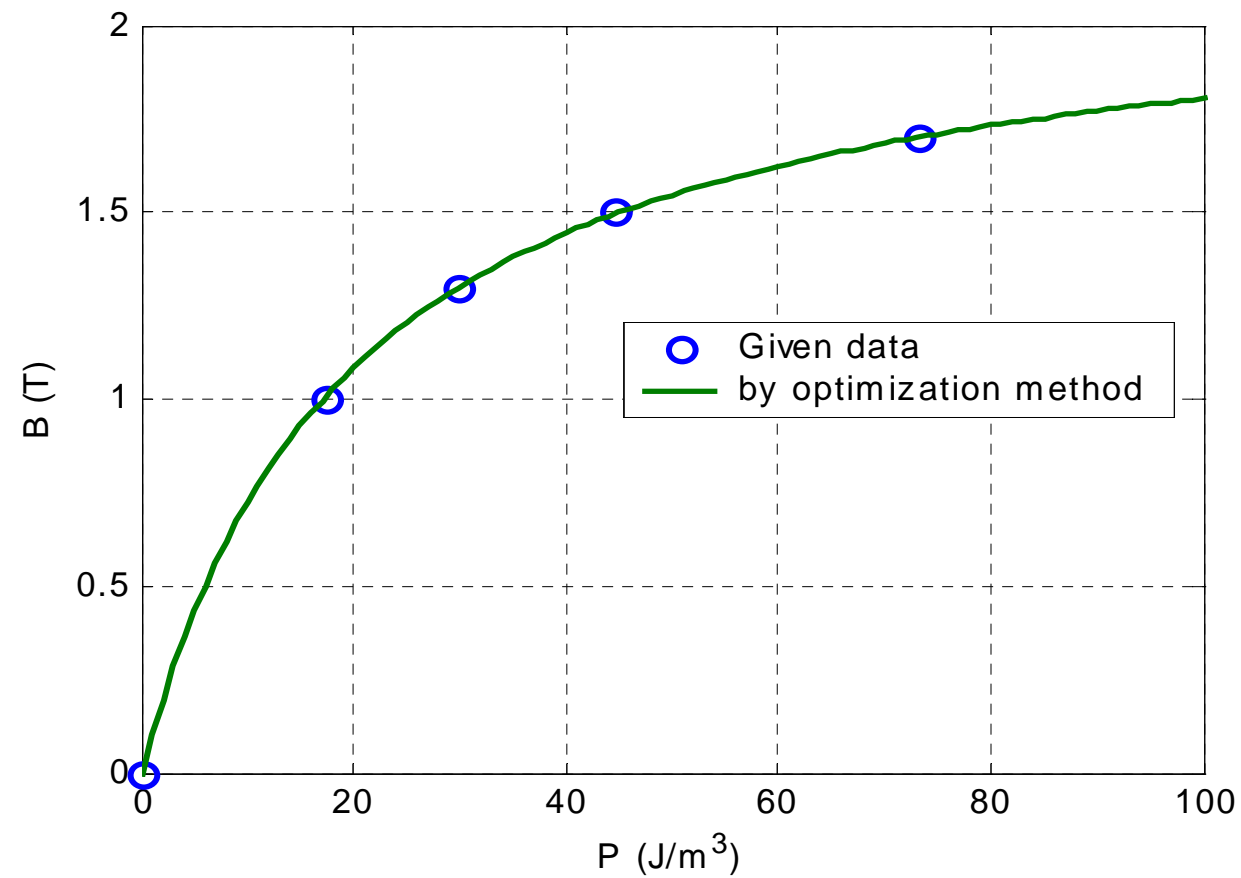

Figure 4.18 Examples of DC Hysteresis Loss Curve using Frolich Equation 


\subsection{Separation of Eddy Current and Hysteresis Losses}

Core losses can be modeled in a simplistic manner as a separate linear resistance in parallel with the nonlinear magnetizing inductance. These losses are proportional to the core volume. From the dimension of the legs and yokes, the volumes can be calculated. If the volumes of legs and yokes are known and the magnitudes of the peak sinusoidal flux in core legs and yokes are known, average core losses that take place in legs and yokes for the applied voltage can be calculated using the relation $\mathrm{P}=\mathrm{V}^{2} / \mathrm{R}$, where $\mathrm{V}$ is the RMS applied voltage. However, this is only valid for steady-state sinusoidal applied voltage of a given RMS magnitude.

Actually the core loss is nonlinear and frequency-dependent and the use of a linear resistance can result in errors for some type of simulations. Therefore, the core loss needs to be modeled using a more sophisticated description. Unfortunately, there is a lack of a suitable nonlinear resistance element in ATP to model the constricted (non-sigmoid, non-monotonic) flux-current loop.

A detailed transformer core model is more complicated, since the model should take into account the nonlinear and the frequency-dependent effects of core loss. The core loss description must be a function of frequency and voltage, and must ultimately be implemented in the time domain in ATP.

The modeling of eddy currents and hysteresis has been approximate and difficult, because of the lack of information. In the approach developed here, parameters for the transformer model are estimated using basic transformer test data and optimization techniques. 
To define the frequency-dependent effects of core loss, the core loss $\left(\mathrm{P}_{\mathrm{C}}\right)$ at a given frequency is generally given as below [17].

$$
P_{C}=P_{H}+P_{E}=\alpha \cdot f+\beta \cdot f^{2} \text { where } P_{H} \text { is hysteresis loss and } P_{E} \text { is eddy current loss }
$$

If the core losses $\left(P_{1}\right.$ and $\left.P_{2}\right)$ at two frequencies $\left(f_{1}\right.$ and $\left.f_{2}\right)$ are given, the coefficients $(\alpha$ and $\beta$ ) for hysteresis loss and eddy current loss are defined as:

$$
\alpha=\frac{P_{1} \cdot f_{2}{ }^{2}-P_{2} \cdot f_{1}{ }^{2}}{f_{1} \cdot f_{2} \cdot\left(f_{2}-f_{1}\right)} \quad \text { and } \quad \beta=\frac{P_{2} \cdot f_{1}{ }^{2}-P_{1} \cdot f_{2}{ }^{2}}{f_{1} \cdot f_{2} \cdot\left(f_{2}-f_{1}\right)}
$$

Generally $P_{E}$ is proportional to $\lambda^{2}$ and $f^{2}$ in the low-frequency range. In the highfrequency range, it changes to about $\mathrm{f}^{1.5}$ because of the skin effect in the laminations. The ratio of $\mathrm{P}_{\mathrm{H}} / \mathrm{P}_{\mathrm{E}}$ is about 3 for silicon steel, about $2 / 3$ for grain-oriented steel and about $1 / 3$ for a modern transformer [17]. If the core loss and the ratio of $\mathrm{P}_{\mathrm{E}} / \mathrm{P}_{\mathrm{C}}$ at $100 \% \mathrm{~V}$ are given, the nonlinear and frequency-dependent core loss at the voltage $\mathrm{V}$ and the frequency $\mathrm{f}$ are defined as:

$$
\begin{aligned}
& P_{E}=\left(\text { ratio of } \mathrm{P}_{\mathrm{E}} / \mathrm{P}_{\mathrm{C}}\right) \cdot P(100 \% \mathrm{~V}) \cdot(\lambda(\mathrm{V}) / \lambda(100 \% \mathrm{~V}))^{2} \cdot(f / 60)^{2} \\
& P_{H}=\left(1-\text { ratio of } \mathrm{P}_{\mathrm{E}} / \mathrm{P}_{\mathrm{C}}\right) \cdot P(100 \% \mathrm{~V}) \cdot(\lambda(\mathrm{V}) / \lambda(100 \% \mathrm{~V}))^{K} \cdot(f / 60) \\
& P_{C}(\text { Core loss @V and })=P_{E}+P_{H}
\end{aligned}
$$

Thus, "k" for hysteresis loss can be calculated from the above equations. " $\mathrm{k}$ " is generally larger than 2 and close to 3 for grain-oriented steel. [17]

Core losses $\left(\mathrm{P}_{\mathrm{C}}\right)$ at $100 \% \mathrm{~V}$ and $110 \% \mathrm{~V}$ are usually given from factory test reports as shown in Table 4.11. Core loss at $120 \mathrm{~Hz}$ and $200 \% \mathrm{~V}$ (frequency and voltage are both 
changed in order to keep the flux magnitude constant) is assumed as $292.7 \mathrm{~kW}(99.2 \mathrm{~kW} \times$ 1.48 / 0.51) from Table 4.12.

Table 4.11 Core Loss from Transformer Factory Test Report

\begin{tabular}{|c|c|c|}
\hline & $100 \% \mathrm{~V}, 60 \mathrm{~Hz}$ & $110 \% \mathrm{~V}, 60 \mathrm{~Hz}$ \\
\hline $\operatorname{Voc}(\mathrm{V})$ & 13,800 & 15,180 \\
\hline$\lambda(\mathrm{Wb}-\mathrm{t})$ & 51.77 & 56.95 \\
\hline $\mathrm{Pc}(\mathrm{W})$ & 99,200 & 134,080 \\
\hline Ratio of Pc @ $100 \% \mathrm{~V}$ & 1.0 & 1.35 \\
\hline $\mathrm{Ic}(\mathrm{rms})$ & 7.188406 & 8.832675 \\
\hline
\end{tabular}

Table 4.12 Core loss for M4 $(B=1.5 T)$ from Manufacture's Catalog [3]

\begin{tabular}{|c|c|c|c|c|c|}
\hline $\begin{array}{c}\text { Frequency } \\
(\mathrm{Hz})\end{array}$ & $60 \mathrm{~Hz}$ & $120 \mathrm{~Hz}$ & $180 \mathrm{~Hz}$ & $300 \mathrm{~Hz}$ & $1000 \mathrm{~Hz}$ \\
\hline Core loss (W/lb) & 0.51 & 1.48 & 2.85 & 6.7 & 56 \\
\hline $\begin{array}{c}\text { Core loss } \\
(\mathrm{W} / \mathrm{kg})\end{array}$ & 1.12 & 3.25 & 6.26 & 14.7 & 123 \\
\hline Ratio @ 60-Hz & 1.0 & 2.95 & 5.7 & 13.4 & 112 \\
\hline
\end{tabular}

Table 4.13 Calculated Core Loss Data from Table 4.11 and Table 4.12

\begin{tabular}{|c|c|c|c|}
\hline & $100 \% \mathrm{~V}, 60 \mathrm{~Hz}$ & $110 \% \mathrm{~V}, 60 \mathrm{~Hz}$ & $200 \% \mathrm{~V}, 120 \mathrm{~Hz}$ \\
\hline Voltage $(\mathrm{rmsV})$ & 13,800 & 15,180 & 27,600 \\
\hline Pc $(\mathrm{W})(\mathrm{pu})$ & $99,200(1.0)$ & $134,080(1.35)$ & $292,700(2.95)$ \\
\hline Rc $($ Ohm) & 1919.8 & 1718.6 & 2602.5 \\
\hline Ic (rmsA) & 7.1884 & 8.8327 & 10.6051 \\
\hline Ic (peakA) & 10.165 & 14.632 & - \\
\hline
\end{tabular}

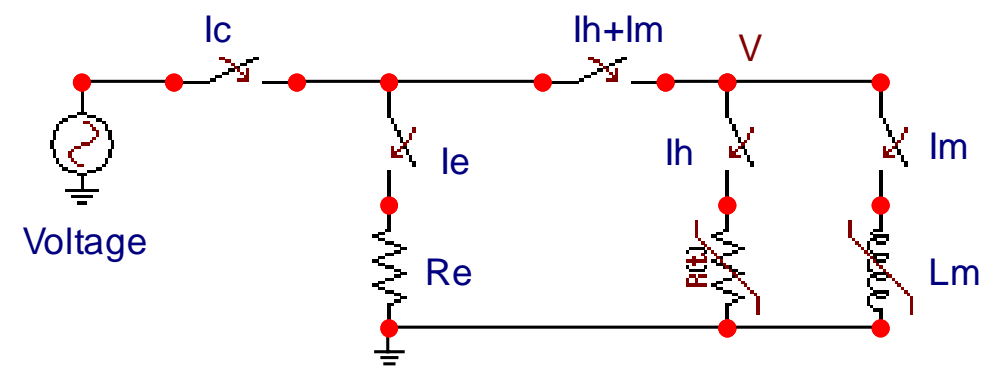

Figure 4.19 Equivalent Circuit for Separated Core Loss Model 
For the model in Figure 4.19, separation of the core losses is necessary as below.

$$
P_{C}(\text { core loss })=P_{H}(\text { hysteresis loss })+P_{E}(\text { eddy current loss })
$$

The ratio between $\mathrm{P}_{\mathrm{E}}$ and $\mathrm{P}_{\mathrm{H}}$ is about 1 to 3 , but is usually not given in a factory test report, since these two parts cannot be separated in the factory's excitation tests.

Let $P_{H}=X_{1} \cdot \lambda^{X 2} \cdot f$ and $P_{E}=X_{3} \cdot \lambda^{2} \cdot f^{X 4}$, then $P_{C}=X_{1} \cdot \lambda^{X 2} \cdot f+X_{3} \cdot \lambda^{2} \cdot f^{X 4}$.

There are four unknowns $\left(\mathrm{X}_{1}, \mathrm{X}_{2}, \mathrm{X}_{3}, \mathrm{X}_{4}\right)$. From Table 4.13, three known conditions are:

$$
\begin{aligned}
1.0=X_{1}+X_{3} & \text { at } \lambda=1 \mathrm{pu} \text { and } f=1 \mathrm{pu}(60 \mathrm{~Hz}) \\
1.35=X_{1} \cdot(1.1)^{X 2}+(1.21) \cdot X_{3} & \text { at } \lambda=1.1 \mathrm{pu} \text { and } f=1 \mathrm{pu} \\
2.95=(2) \cdot X_{1}+X_{3} \cdot(2)^{X 4} & \text { at } \lambda=1 \mathrm{pu} \text { and } f=2 \mathrm{pu}
\end{aligned}
$$

To find the solutions for Equations (4.21) through (4.23), the optimization techniques can be used. One method is the successive LP method. Linearizing the objective function and the nonlinear equality constraint function at $\mathrm{X}=\mathrm{a}$,

$$
F(X)=F(a)+\nabla F(a) \cdot(X-a) \text { and } h(X)=h(a)+\nabla h(a) \cdot(X-a)
$$

Then the functions are linear and LP gives the solution at each iteration.

Case 1: By Successive LP method and Finite Difference Approximation

$$
\begin{aligned}
& F(X)=\left(X_{1} \cdot(1.1)^{X 2}+1.21 \cdot X_{3}-1.35\right)^{2}+\left(2 \cdot X_{1}+X_{3} \cdot(2)^{X 4}-2.95\right)^{2} \text { as objective function. } \\
& 1=X_{1}+X_{3} \quad \text { as linear constraint. }
\end{aligned}
$$

Case 2: By Successive LP method and Finite Difference Approximation 


$$
\begin{array}{ll}
F(X)=\left(2 \cdot X_{1}+X_{3} \cdot(2)^{X 4}-2.95\right)^{2} & \text { as objective function. } \\
1=X_{1}+X_{3} & \text { as linear constraint. } \\
1.35=X_{1} \cdot(1.1)^{X 2}+1.21 \cdot X_{3} & \text { as nonlinear constraint. }
\end{array}
$$

However, using Successive LP method did not give convergence in either case as shown in Figure 4.20. There are many local optima for Equations (4.22) and (4.23) can be seen in Figure 4.21.

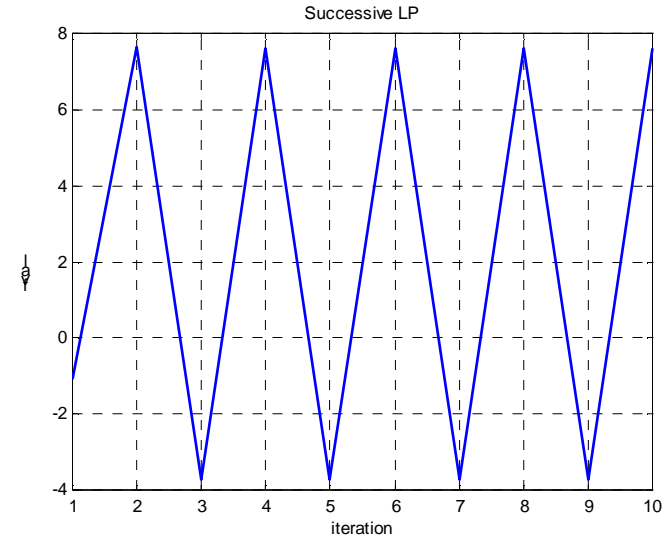

left : Case 1

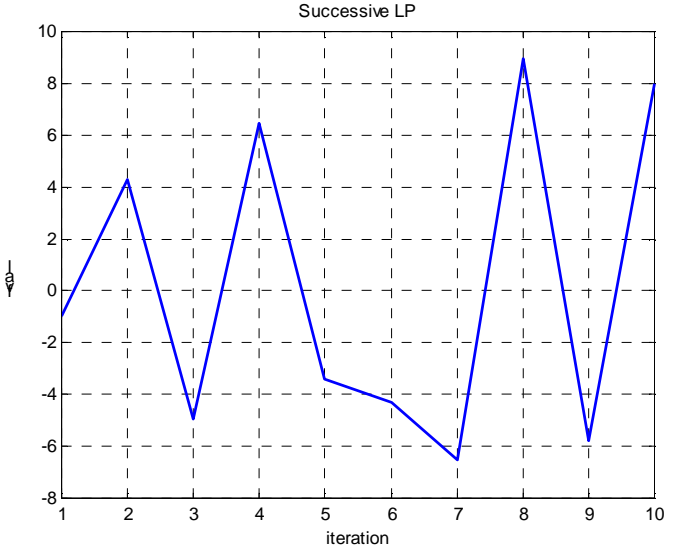

right : Case 2

Figure 4.20 Function Values at Each Iteration by Successive LP method
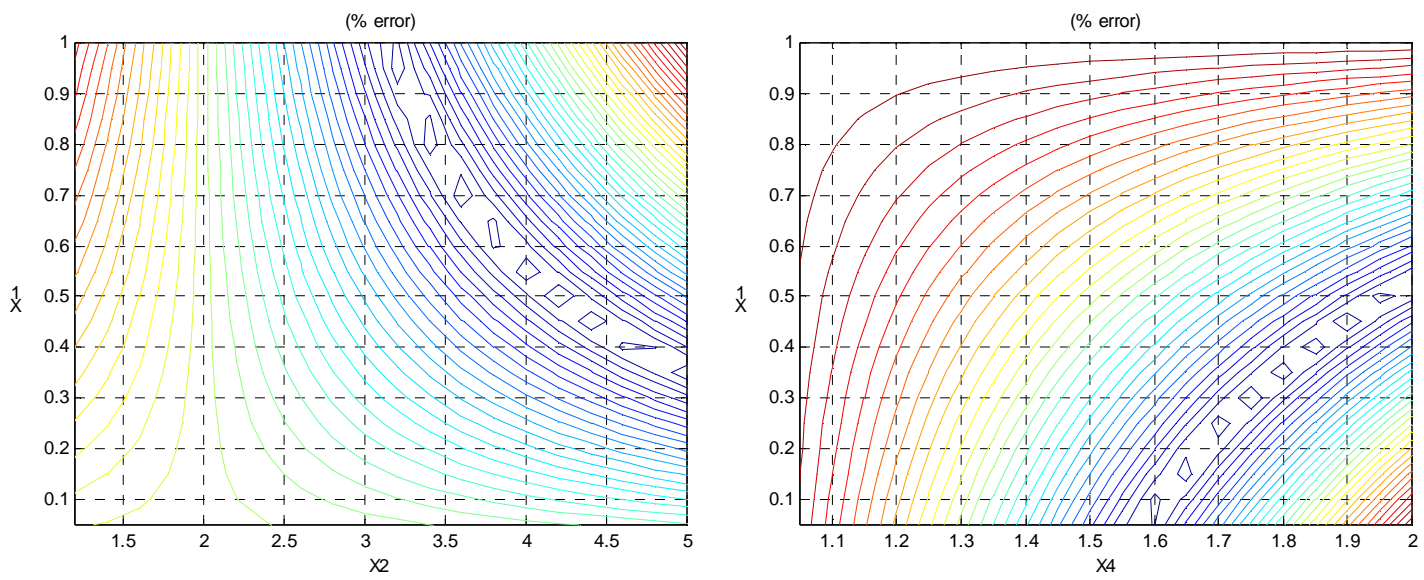

Figure 4.21 Local Optima for Equation 4.22 (left) and Equation 4.23 (right) 
Next, applying “Fmincon" of MATLAB ${ }^{\circledR}$ Optimization tool box (Section 2.6.2) to find the solutions of a constrained nonlinear multivariable function,

Case 1:

$$
F(X)=\left(X_{1}-X_{3}-1\right)^{2}+\left(X_{1} \cdot(1.1)^{X 2}+1.21 \cdot X_{3}-1.35\right)^{2}+\left(2 \cdot X_{1}+X_{3} \cdot 2^{X 4}-2.95\right)^{2} \text { as objective }
$$

function.

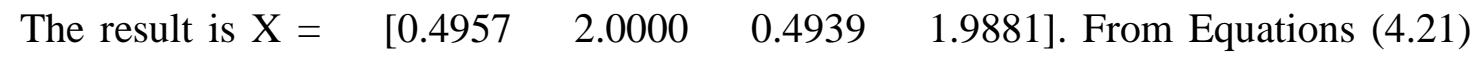
$\sim(4.23), \mathrm{P}_{\mathrm{C}}=\left[\begin{array}{lll}0.9896 & 1.1974 & 2.9506\end{array}\right]$ and \%error $=\left[\begin{array}{llll}1.0432 & 15.4235 & -0.0001\end{array}\right]$

Case 2:

$$
\begin{aligned}
& F(X)=\left(X_{1} \cdot(1.1)^{X 2}+1.21 \cdot X_{3}-1.35\right)^{2}+\left(2 \cdot X_{1}+X_{3} \cdot 2^{X 4}-2.95\right)^{2} \text { as objective function } \\
& 1=X_{1}+X_{3} \text { as linear constraint }
\end{aligned}
$$

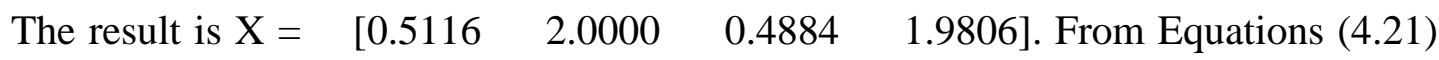
$\sim(4.23), \mathrm{P}_{\mathrm{C}}=\left[\begin{array}{lll}1.0000 & 1.2100 & 2.506\end{array}\right]$ and \%error=[ $\left.\begin{array}{lll}0.0000 & 14.1613 & 0.0000\end{array}\right]$

Case 3:

$$
\begin{aligned}
& F(X)=\left(2 \cdot X_{1}+X_{3} \cdot 2^{X 4}-2.95\right)^{2} \text { as objective function to minimize } \\
& 1=X_{1}+X_{3} \text { as linear constraint } \\
& 1.35=X_{1} \cdot(1.1)^{X 2}+1.21 \cdot X_{3} \text { as nonlinear constraint. }
\end{aligned}
$$

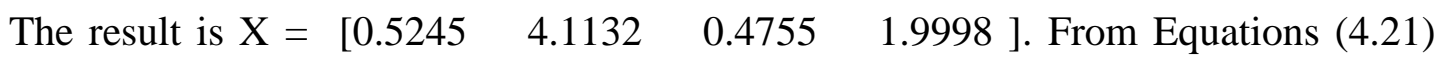
$\sim(4.23), \mathrm{P}_{\mathrm{C}}=\left[\begin{array}{lll}1.0000 & 1.3516 & 2.9506\end{array}\right]$ and \%error $=\left[\begin{array}{lll}0.0000 & 0.0000 & 0.0000\end{array}\right]$

The Case 3 gives the best result. Using the above result, separated loss functions for the example transformer can be obtained: 


$$
\begin{aligned}
& P_{H}=0.5245 \cdot \lambda^{4.1132} \cdot f \text { and } P_{E}=0.4755 \cdot \lambda^{2} \cdot f^{1.9998} \quad(p u) \\
& R_{E}=V^{2} / P_{E}=\lambda^{2} \cdot f^{2} /\left(0.4755 \cdot \lambda^{2} \cdot f^{1.9998}\right) \approx 2.103 \quad(p u)=4037.3 \mathrm{ohms} \\
& R_{H}=V^{2} / P_{H}=\lambda^{2} \cdot f^{2} /\left(0.5245 \cdot \lambda^{4.1132} \cdot f\right)=1.9066 \cdot \lambda^{-2.1132} \cdot f \quad(p u) \\
& I_{H}=P_{H} / V=\left(0.5245 \cdot \lambda^{4.1132} \cdot f\right) /(\lambda \cdot f)=0.5245 \cdot \lambda^{3.1132} \quad(p u) \\
& I_{E}=V / R_{E}=V / 2.103(p u)
\end{aligned}
$$

Table 4.13 Calculated Core Loss using functions

\begin{tabular}{|c|c|c|c|}
\hline & $100 \% \mathrm{~V}, 60 \mathrm{~Hz}$ & $110 \% \mathrm{~V}, 60 \mathrm{~Hz}$ & $200 \% \mathrm{~V}, 120 \mathrm{~Hz}$ \\
\hline Voltage $(\mathrm{V}, \mathrm{rms})$ & 13,800 & 15,180 & 27,600 \\
\hline $\mathrm{P}_{\mathrm{C}}(\mathrm{W}$ and pu) & $99,200(1.0)$ & $134,080(1.35)$ & $292,710(2.95)$ \\
\hline $\mathrm{P}_{\mathrm{H}}(\mathrm{W}$ and pu) & $52,030(.5245)$ & $77,000(.7763)$ & $104,060(1.0490)$ \\
\hline $\mathrm{P}_{\mathrm{E}}(\mathrm{W}$ and pu) & $47,170(.4755)$ & $57,080(.5754)$ & $188,650(1.9017)$ \\
\hline $\mathrm{I}_{\mathrm{C}}(\mathrm{A}, \mathrm{rms})$ & 7.1884 & 8.8326 & 10.6055 \\
\hline $\mathrm{I}_{\mathrm{H}}(\mathrm{A}, \mathrm{rms})$ & 3.7703 & 5.0727 & 3.7703 \\
\hline $\mathrm{I}_{\mathrm{E}}(\mathrm{A}, \mathrm{rms})$ & 3.4181 & 3.7599 & 6.8352 \\
\hline
\end{tabular}

In the case of $X_{4}=2$, the eddy current loss $\left(P_{E}\right)$ can be modeled by a resistance $R_{E}$. In the case of $\mathrm{X}_{4} \neq 2$, frequency dependency needs to be considered. Hysteresis loss $\mathrm{P}_{\mathrm{H}}$ can be represented by a resistance $\left(R_{H}\right)$ in Equation (4.26). In this case, $R_{H}$ for hysteresis loss is nonlinear and frequency dependent. Therefore, the resistance should be replaced by a frequency-dependent resistance $R_{H}(f)$.

However, $\mathrm{I}_{\mathrm{H}}$ is nonlinear and frequency independent. If the hysteresis loss can be modeled by $\mathrm{I}_{\mathrm{H}}$ current injection, the frequency-dependency can be implemented as a timevarying current injection. The enclosed area of a $\lambda-i_{H}$ plot shown in Figure 4.22 is the hysteresis loss per one cycle. Hysteresis loss at rated frequency might be represented by a two-slope $v$-i curve, defined by Figure 4.22. If the RMS currents of the example transformer are converted to the peak currents by the SATURATION routine, $\mathrm{I}_{\mathrm{H}}$ is 5.332 peak-A at 
$100 \% \mathrm{~V}$ and 9.179 peak-A at $110 \% \mathrm{~V}$. Figure 4.23 shows the waveforms for the $v-i_{H}$ and $\lambda-i_{H}$ at $110 \% \mathrm{~V}$ and $60 \mathrm{~Hz}$. However, as seen in Figure 4.24, actual hysteresis loss is dependent on maximum flux, not voltage. This illustrates the difficulties and errors encountered if average power descriptions are used to develop time-domain representations [34].

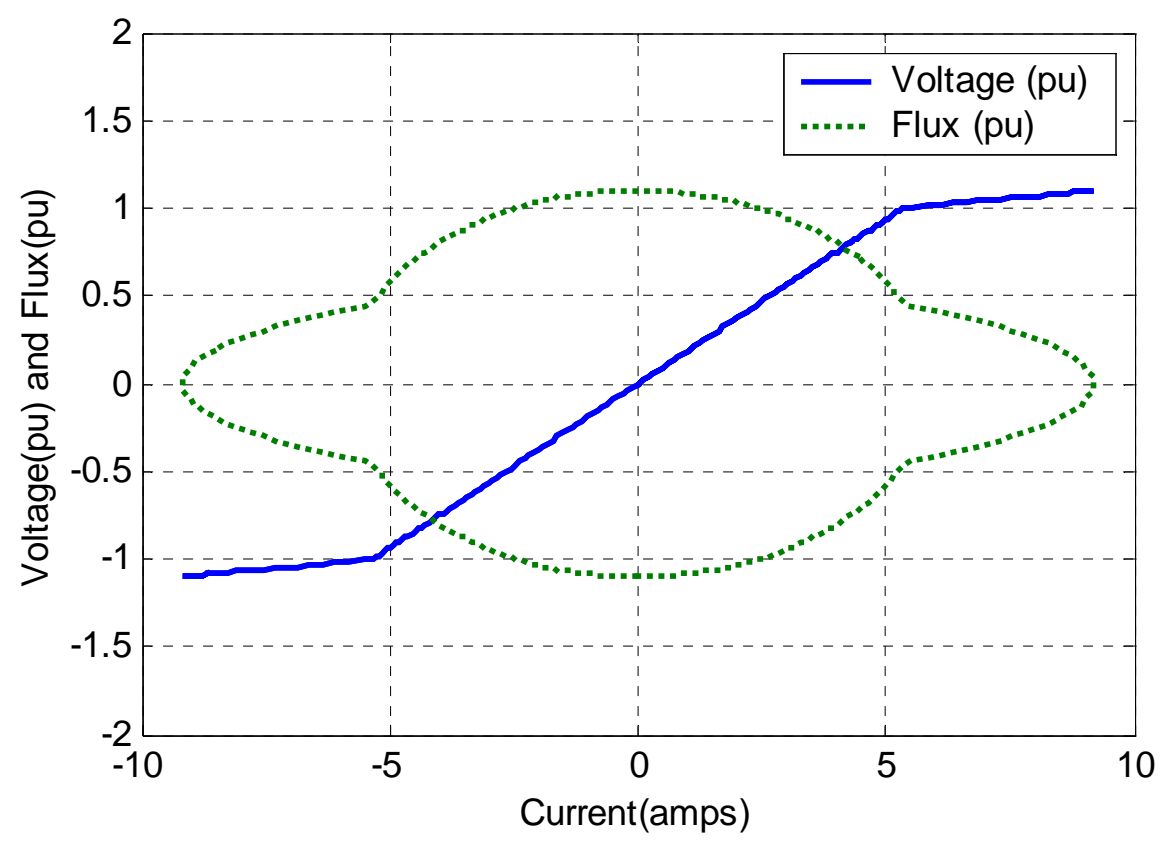

Figure 4.22 $v-i_{H}$ and $\lambda-i_{H}$ Plot at $110 \% \mathrm{~V}$ and $60 \mathrm{~Hz}$ 


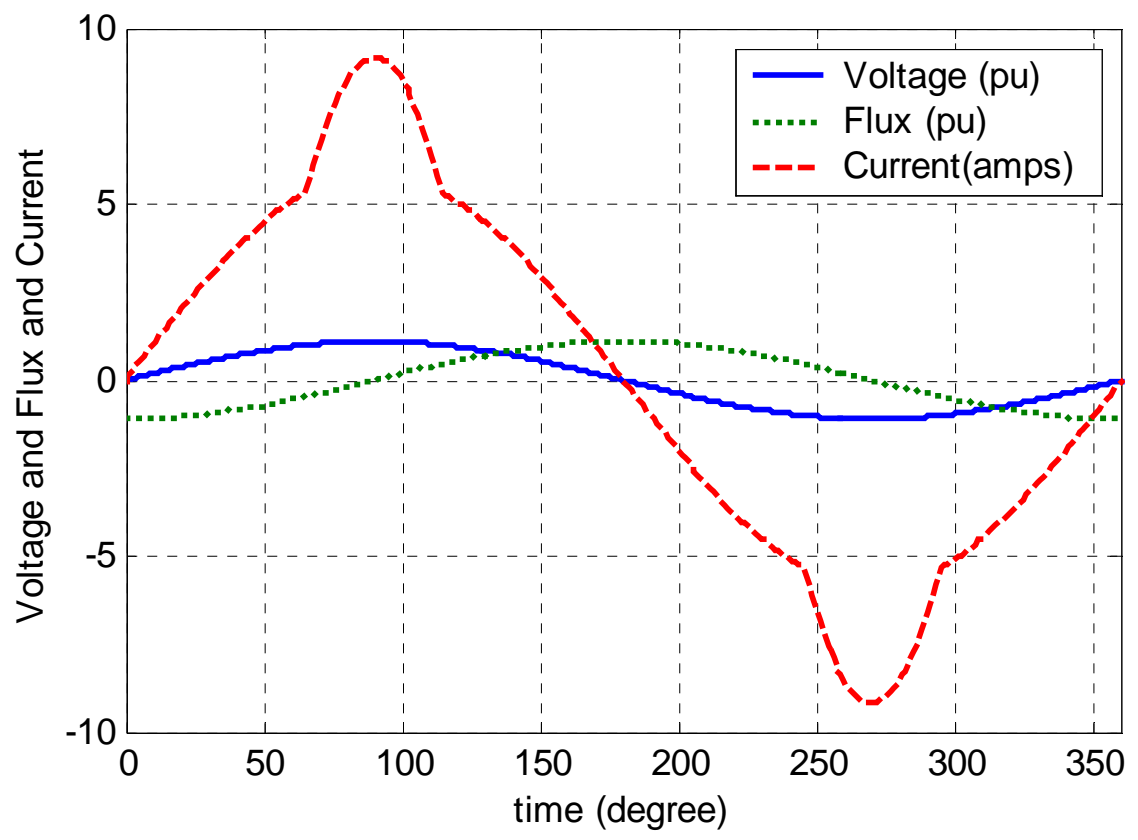

Figure 4.23 Time vs $v, \lambda, i_{H}$ Waveforms at $110 \% \mathrm{~V}$ and $60 \mathrm{~Hz}$

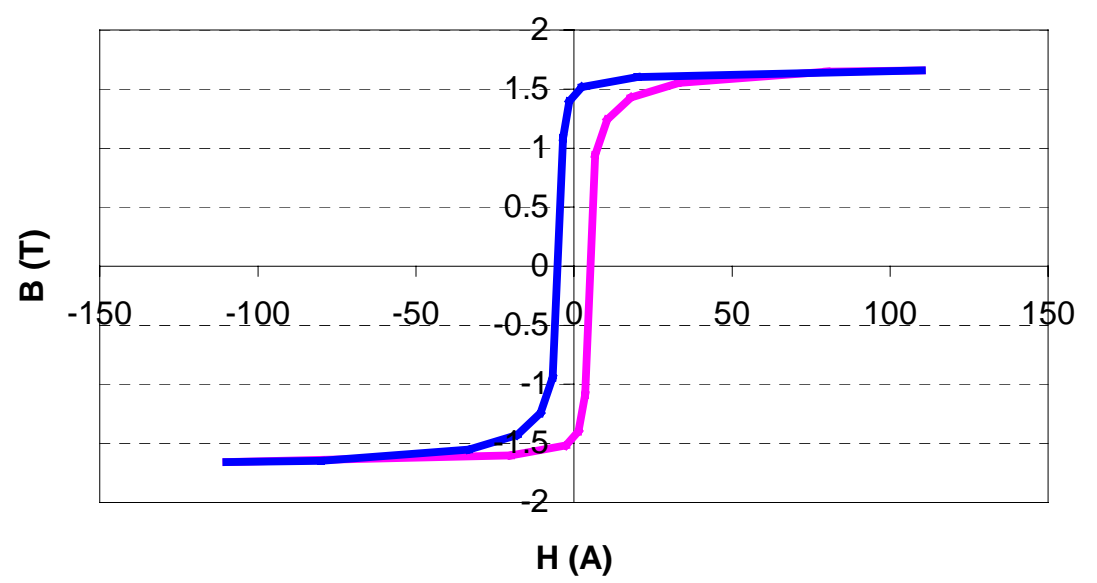

Figure 4.24 Typical Hysteresis Loop 


\subsection{Hysteresis Loop Model}

The $\lambda-i$ hysteresis loop gives the instantaneous relationship between current and flux linked for near-DC periodic excitation. Recall that $\lambda-i$ can be obtained by scaling the B-H characteristic. The spine of the $\lambda-i$ hysteresis loop gives the normal magnetic saturation curve shown in Figure 4.25. (In various references, the normal saturation curve is also referred to as the "initial," "DC," or "virgin" saturation curve). Hysteresis loss can be thought of as a nonlinear frequency-dependent resistance. Hysteresis loss is not directly a function of voltage, but of flux linked. Therefore, the matching of average losses for $60 \mathrm{~Hz}$ excitation does not mean that correct flux-current trajectory is being followed in the time domain. Residual flux of a transformer is another important aspect, critical for inrush simulations. Therefore, a correct hysteresis loop trajectory is a necessary part of a correct time domain core model. Note that $\left(\mathrm{H}_{\text {ctop }}, \mathrm{B}_{\text {ctop }}\right)$ is defined here as the coercive force and flux density corresponding to the maximum known excitation level.
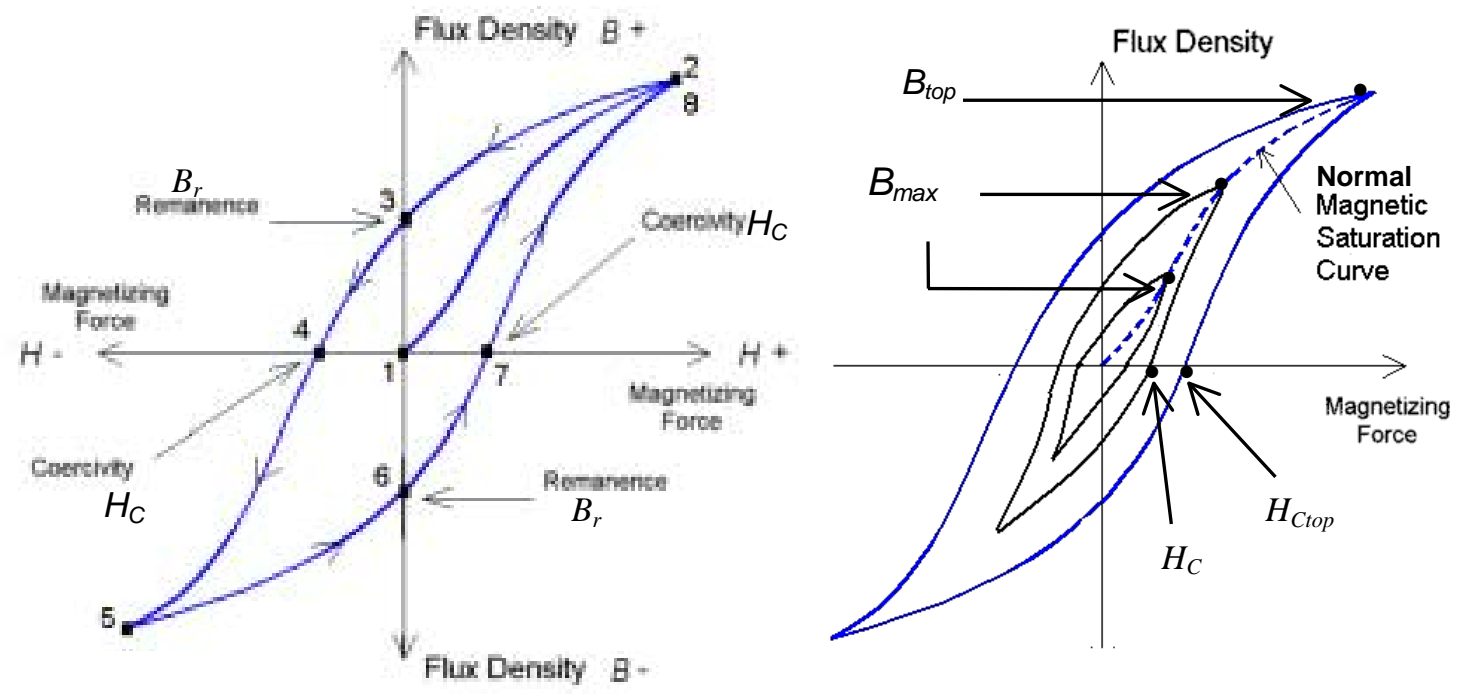

Figure 4.25 Example of Hysteresis Loop [22] 
One method of hysteretic loop representation is Equation (4.29), which uses two hyperbolic functions. The resulting loop is shown in Figure 4.26. However, these functions require $\mathrm{B}_{\mathrm{r}}, \mathrm{B}_{\text {sat }}$, and $\mathrm{H}_{\mathrm{C}}$ defined for each loop [51].

$$
B_{+}=B_{s a t} \times \frac{H+H_{C}}{\left|H+H_{C}\right|+H_{C} \cdot\left(\frac{B_{s a t}}{B_{r}}-1\right)} \text { and } B_{-}=B_{s a t} x \frac{H-H_{C}}{\left|H-H_{C}\right|+H_{C} \cdot\left(\frac{B_{s a t}}{B_{r}}-1\right)}
$$

where $B_{\text {sat }}$ :Induction at saturation $(T), B_{r}:$ Remanence $(T), H_{C}$ : Coercive force $(A / m)$

From $\mathrm{B}_{+}$and $\mathrm{B}_{-}$, the anhysteretic curve is defined as $\mathrm{B}($ anhysteretic $)=\frac{B_{+}+B_{-}}{2}$.

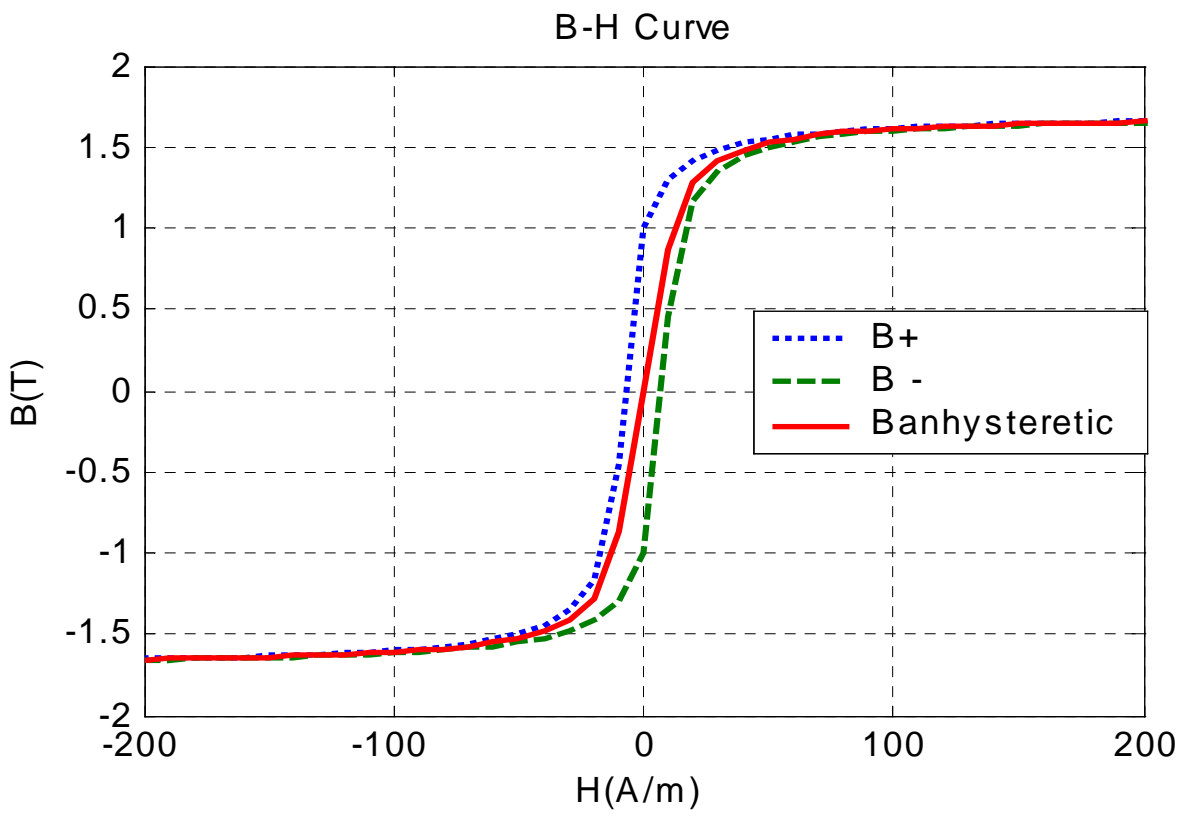

Figure 4.26 Examples of Hysteresis Loop Using Two Hyperbolic Functions

In this work, the $\lambda$-i hysteretic loop is based on the saturation curve given from open circuit test in Section 4.3. The loop is modeled by the left and right displacement. The enclosed area for each cycle is the energy loss from hysteresis. Multiplying this area by the frequency results in the power loss. As a check, the area of the $\lambda-i$ loop for a given $\lambda_{\max }$ should equal the average power loss at the given $\lambda_{\max }$. 
From Section 4.3, the approximation for the saturation curve is given by Equation (4.30). From the saturation data for Armco M4 Steel, "a" and "b" were obtained as 4.2776 and 0.5435 .

$$
H=\frac{a \cdot B}{1-B \cdot b}
$$

As mentioned in Section 4.4, the equation for DC hysteresis loss can be given as Equation (4.31). From the core loss data for Armco M4 Steel, "c" and "d" were obtained as 9.2071 and 0.4326 .

$$
P_{H}=\frac{c \cdot B}{1-B \cdot d}
$$

The right displacement (i.e. the right curve of hysteresis loop minus the core saturation curve at $\mathrm{B}>0$ ) is linear and is assumed as Equation (4.33). The left displacement (i.e. the left curve of hysteresis loop minus the core saturation curve at $\mathrm{B}>0$ ) is nonlinear and increases slowly for low flux, more speedy for bigger flux, and decays to zero for maximum flux $\mathrm{B}_{\max }$ [54]. Thus, the left displacement is assumed as Equation (4.34). At zero flux, both displacements must be the same. This is a coercive force $\left(\mathrm{H}_{\mathrm{C}}\right)$ and is assumed as Equation (4.32) because of its nonlinearity (see Figure 4.27). The coercive force for each loop should be determined to meet the power loss at the $\mathrm{B}_{\max }$ given for the each loop in Equation (4.35). In case of Figure 4.27 from ARMCO M4 [3], approximation using an exponential fit, "K" for Equation (4.32) is about 0.5.

$$
\begin{aligned}
& \text { Coercive force } H_{C}=\left(B_{\max } / B_{\text {top }}\right)^{K} \times H_{\text {ctop }} \\
& \text { Right displacement } R H D=(1-f) * H_{C} \\
& \text { Left displacement } \left.L H D=-H_{C} \cdot(a+1 / a) /[(1-f) / a+a /(1-f)]\right)
\end{aligned}
$$


Power Loss at each loop $=\int_{0}^{B_{\max }} 2 \cdot(R H D-L H D) d B$

where

$B_{\text {max }}=$ Maximum Flux density at each minor loop

$B_{\text {top }}=$ Maximum Flux density for major loop

$a=\left(B_{\text {top }}-B_{\text {max }}\right) / B_{\text {top }}$ and $f=B / B_{\text {max }}$

$H_{\text {ctop }}=$ Maximum Coercive force for major loop

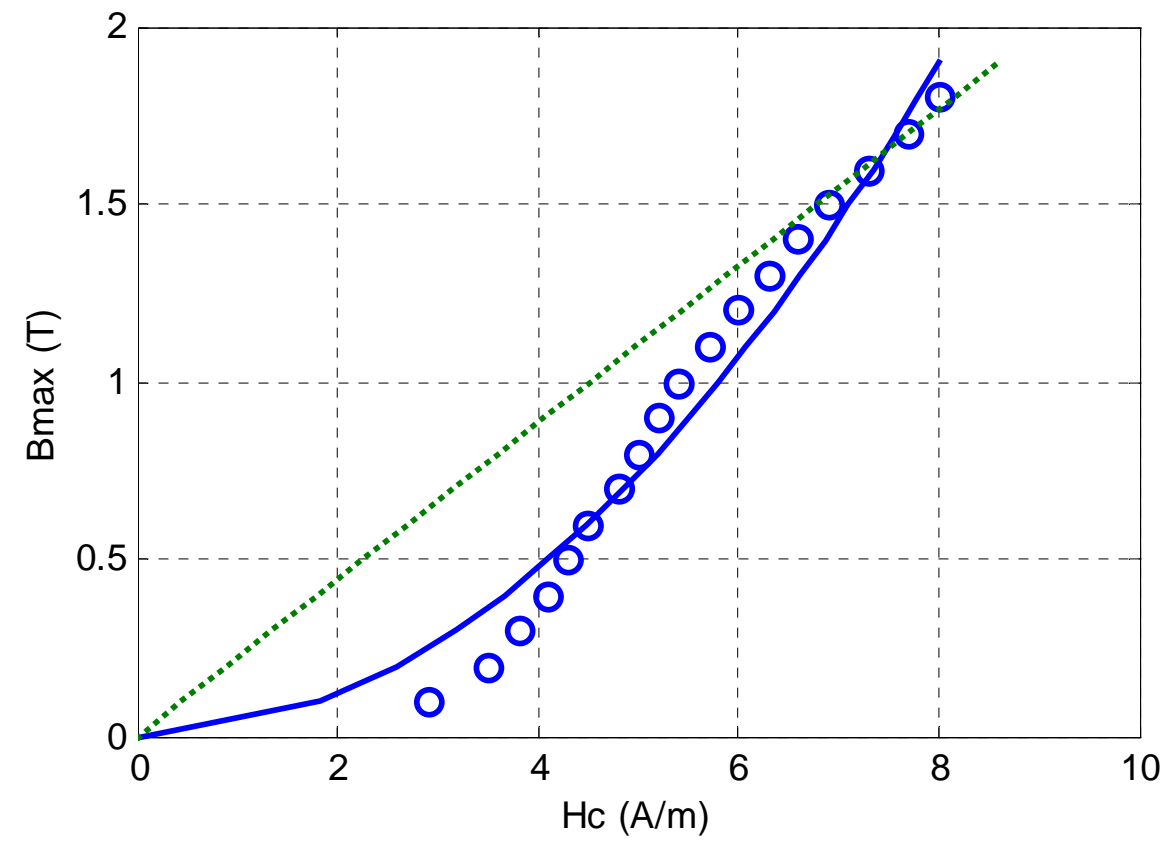

Figure 4.27 $\mathrm{H}_{\mathrm{C}}$ and $\mathrm{B}_{\max }($ dotted line=linear, bold line=square root $)$

Using Equations (4.33) and (4.34), the displacements for each $\mathrm{B}_{\max }$ are shown in Figure 4.28 and the obtained hysteresis loop for B > 0 is shown in Figure 4.29. The entire DC hysteresis loop is shown in Figure 4.30 and the hysteresis loop generated by decaying B with time is shown in Figure 4.31. 


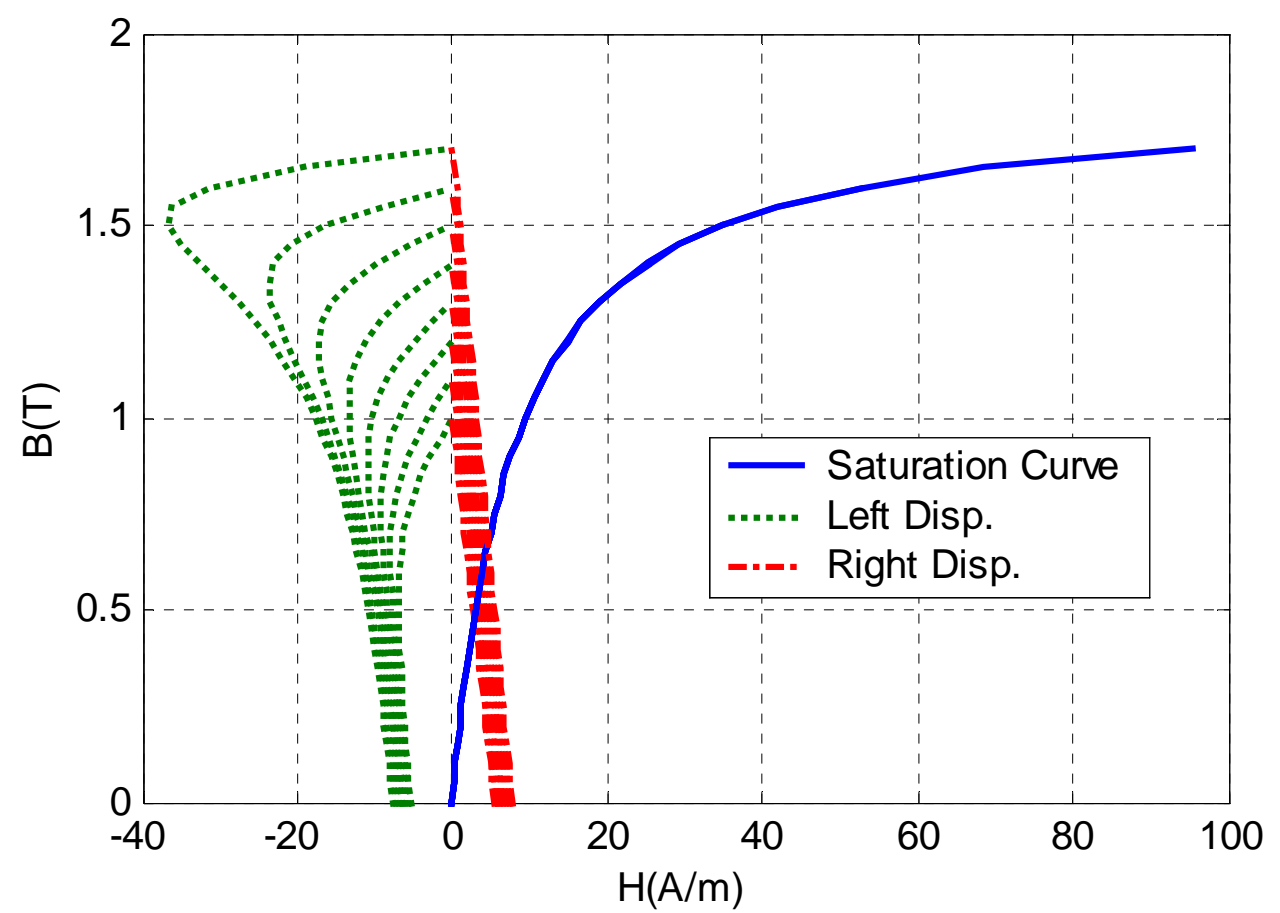

Figure 4.28 Left and Right Displacements of Resistive Hysteresis Current

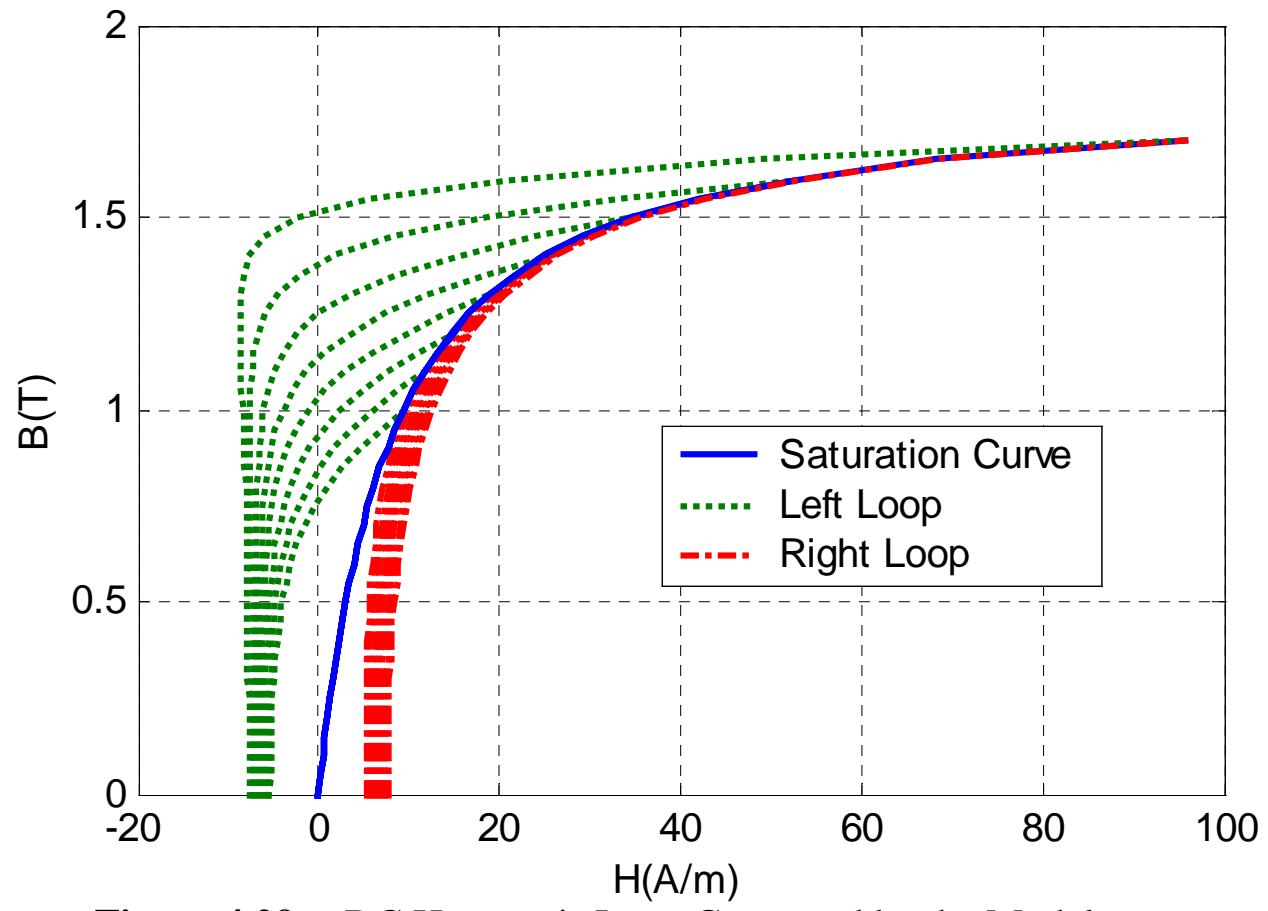

Figure 4.29 DC Hysteresis Loop Generated by the Model 


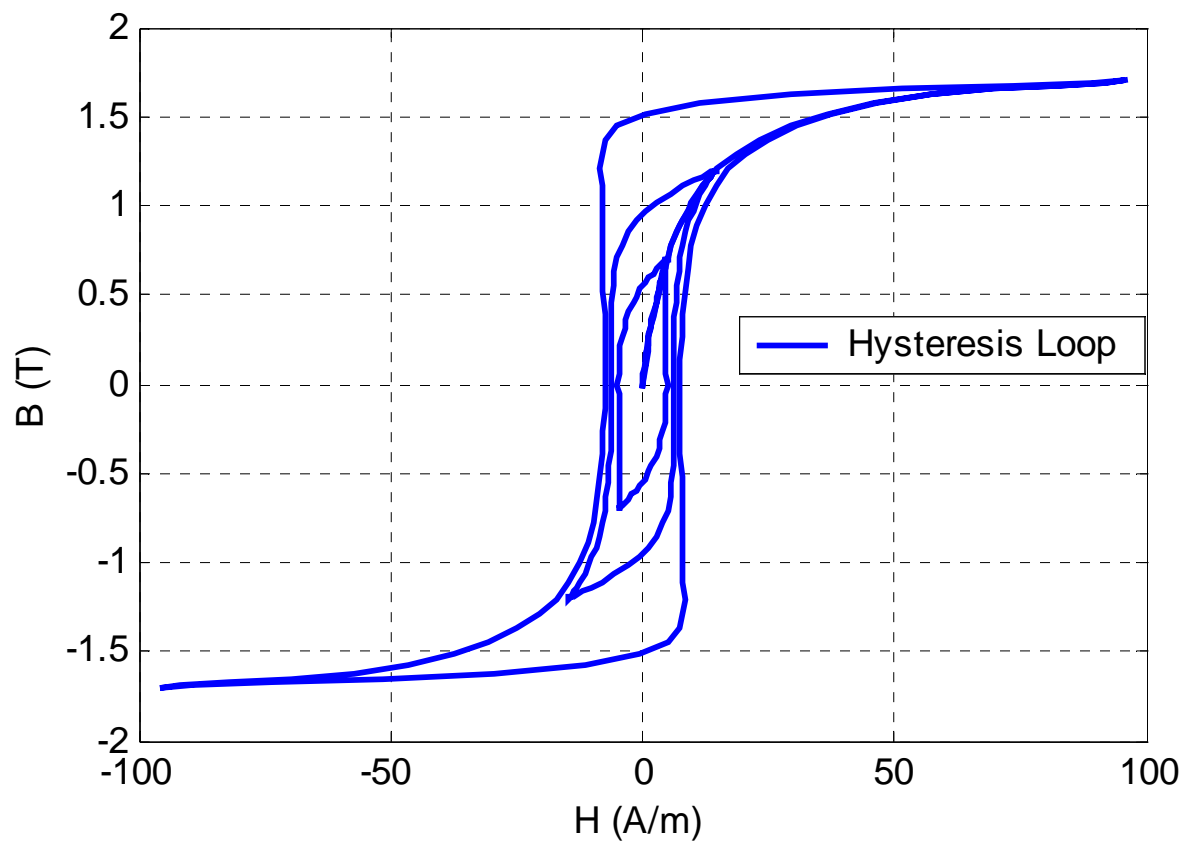

Figure 4.30 DC Hysteresis Loop Generated by the Model

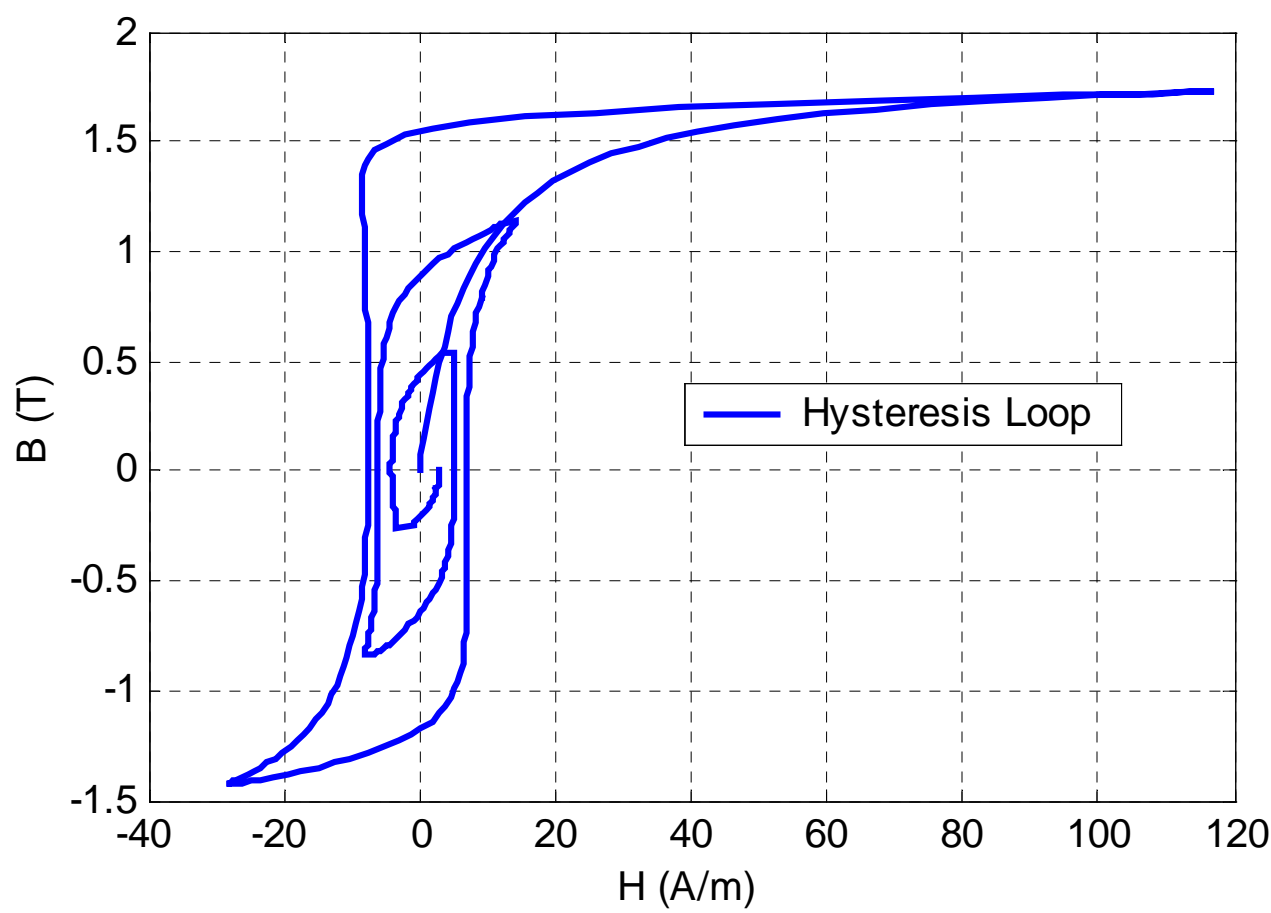

Figure 4.31 Hysteresis Loop Generated by Decaying B with Time 


\section{ATP Implementation of the Model}

Finally, the complete core model implemented in ATP is shown in Figure 4.32. The block diagram related to TACS code is shown in Figure 4.33. "L_sat" represents the anhysteretic saturation curve and is modeled using a Type-93 or a Type-98 element. "I_eddy" and "I_hyster" are modeled using a Type-60 current source controlled by TACS.

"I_hyster" represents the resistive hysteresis current for DC hysteresis loss. The left (or right) displacements of resistive hysteresis current are changed with the right (or left) displacements at the reversing point of flux linkages. The sign of the displacement current is determined by the sign of the flux.

"I_eddy" represents the resistive current for the eddy current loss of core. This current is approximated by dividing a given voltage by a linear resistance using TACS. This implementation is more flexible for future enhancements and avoids unwanted interactions between components, which may occurs when a linear resistor is used.

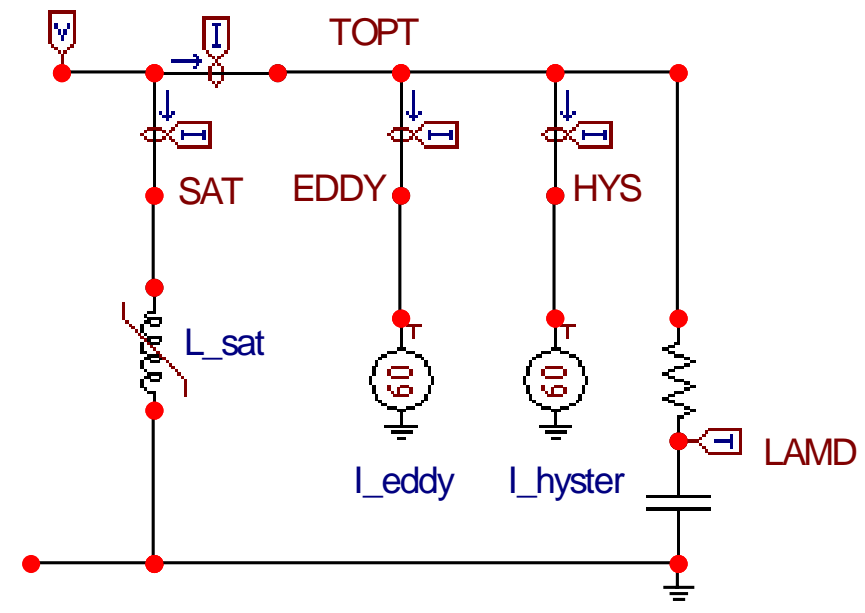

Figure 4.32 Core Model for ATP Implementation 


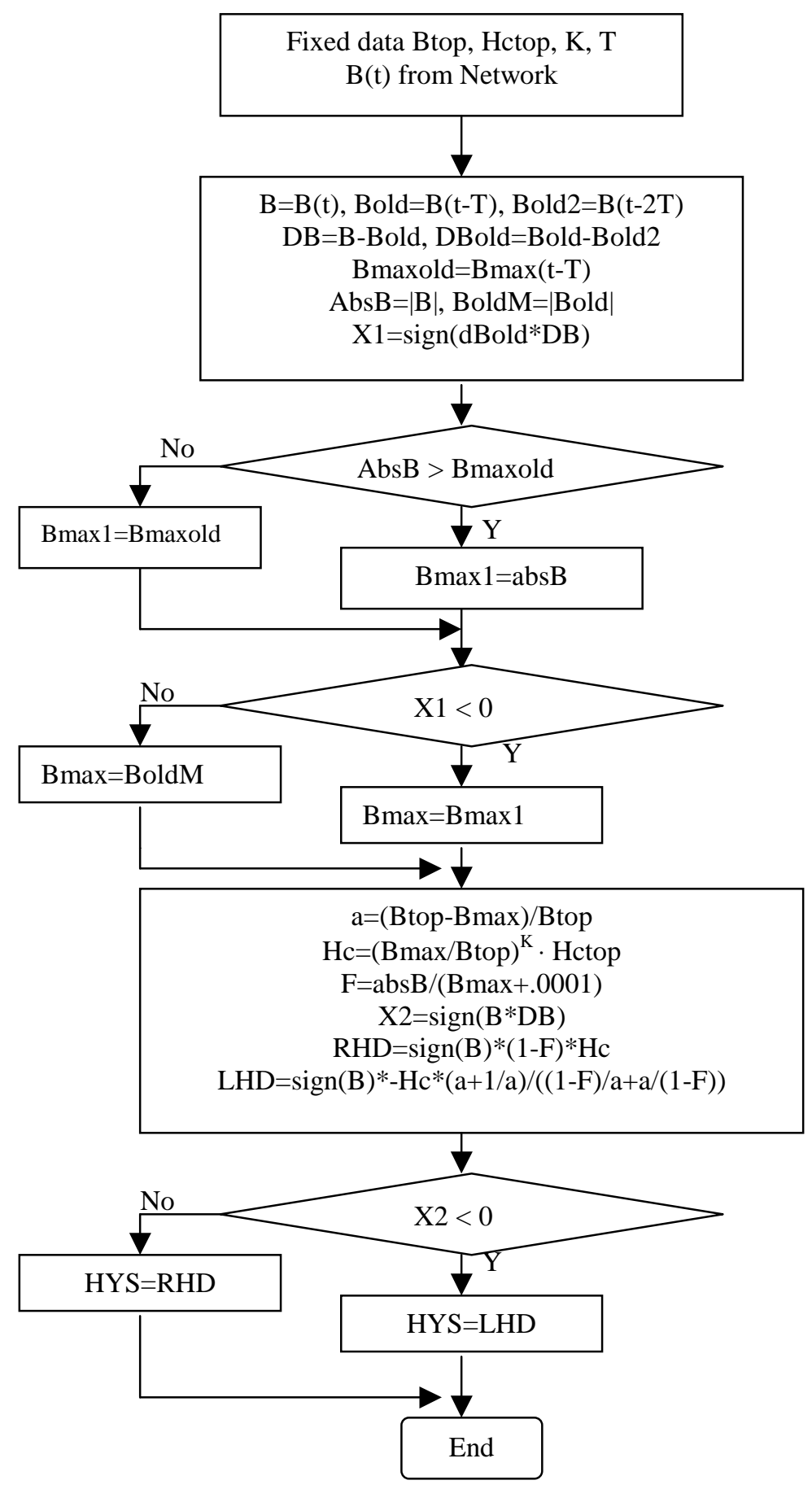

Figure 4.33 Block Diagram for DC Hysteresis Loop using TACS 
Figure 4.34 shows a DC hysteresis loop made by the core model implemented in ATP.

Figure 4.35 shows hysteresis loops generated by decaying B with time.

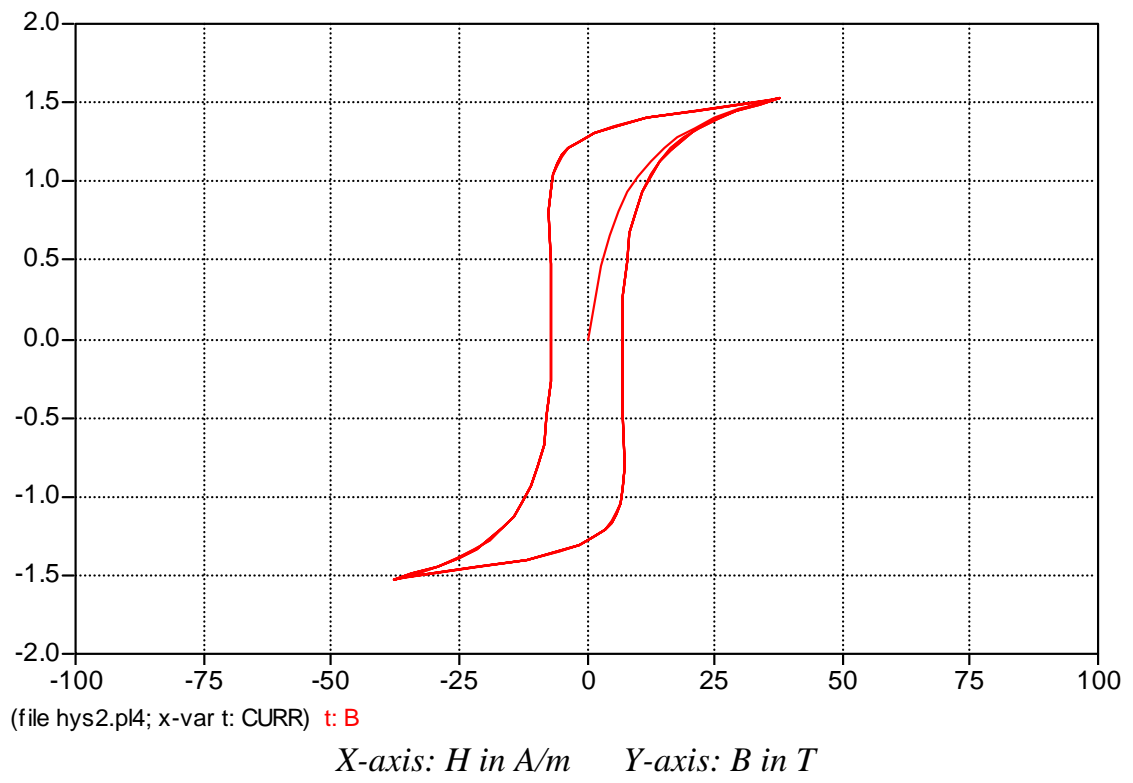

Figure 4.34 DC Hysteresis Loop Generated by the Model

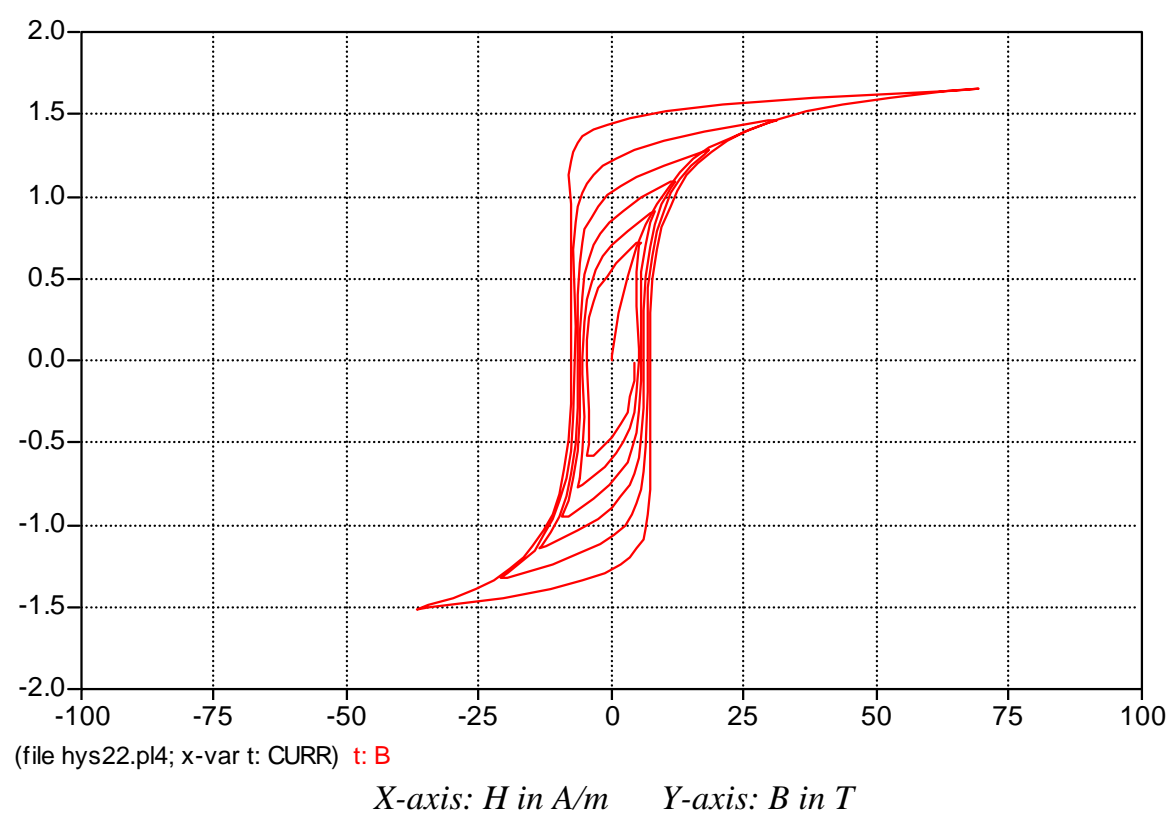

Figure 4.35 DC Hysteresis Loop Generated by Decaying B with Time 


\section{CHAPTER 5 \\ DUALITY - DERIVED MODEL FOR THREE-PHASE TRANSFORMER}

This chapter presents the duality-based equivalent circuit models of three-phase five-

legged, three-phase three-legged, and three-phase shell-form autotransformers for ATP implementation. The equivalent circuits resulting from duality transformations are a topologically-correct lumped-parameter representation.

\subsection{Five-Legged Core Transformer}

Five-legged core transformers are manufactured in cases where a lower transformer height is required, or where it is important to provide a flux return path for related third harmonics. Since the top and bottom yokes are not large enough in cross section to carry all the flux from one leg, the actual flux paths are uncertain and calculation of the core loss is complicated. The yokes saturate and force excess flux to spill over into the outer legs.

The first step is to convert the actual core and coil structure in Figure 5.1 to an approximate lumped-parameter circuit, as shown in Figure 5.2. The windings are represented by MMF sources. The reluctances due to the flux through the iron core are saturable and are represented by solid rectangles, whereas the reluctances due to leakage fluxes through the gaps between windings are linear and are represented by outlined rectangles. The method of duality transformation breaks the core down into separate leg and yoke segments. Elements named $\mathfrak{R}_{\mathrm{L}}$ are the reluctances due to core legs and elements named $\mathfrak{R}_{\mathrm{O}}$ are the reluctances 
due to outer legs. Between the three core legs, there are yokes shown as $\mathfrak{R}_{\mathrm{Y}}$. Leakages between the windings are represented by linear reluctances $\Re_{\mathrm{TL}}, \mathfrak{R}_{\mathrm{CT}}, \mathfrak{R}_{\mathrm{SC}}$.

The next step is to convert the magnetic circuit into the equivalent lumped-parameter electrical circuit as shown in Figure 5.3. Each MMF source and reluctance is replaced by its electrical dual and connected between the neighboring nodes. Note that the MMFs resulting from the duality transformation are replaced with ideal coupling transformers and winding resistances have been added. More details on this will be provided in Section 6.1.5.

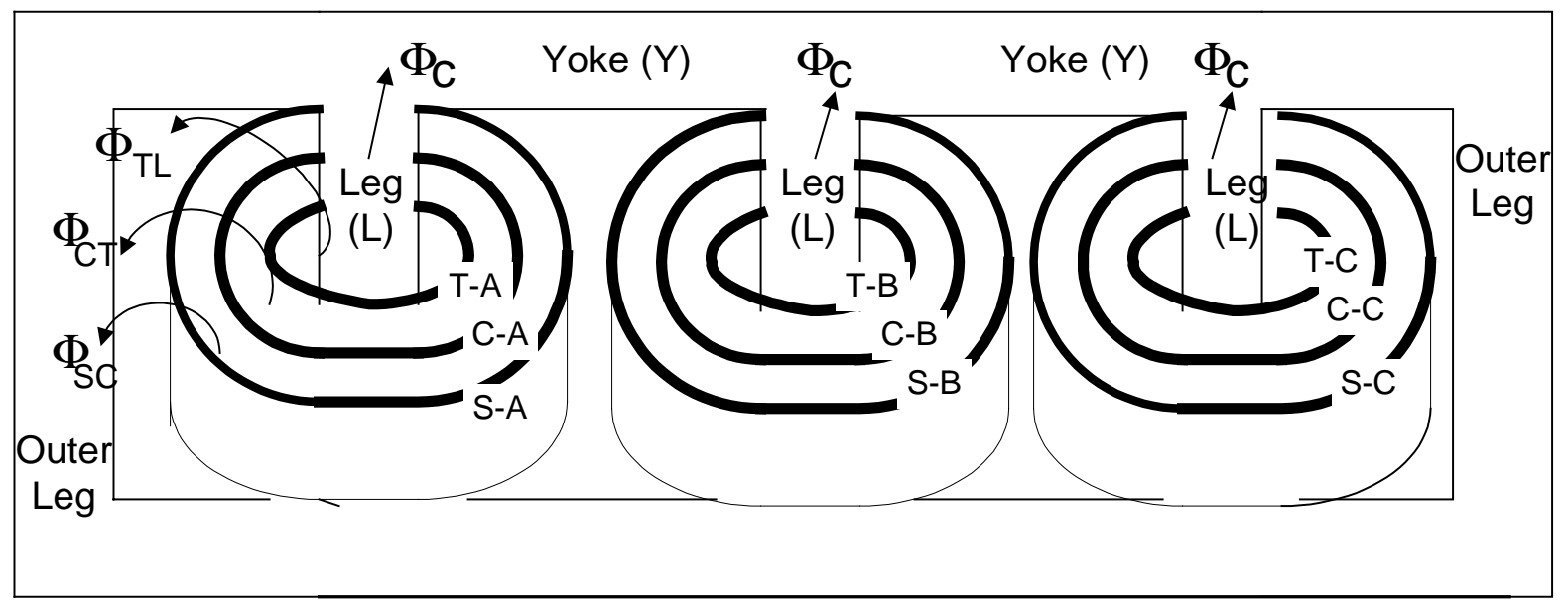

Figure 5.1 Five-legged Core Transformer Structure

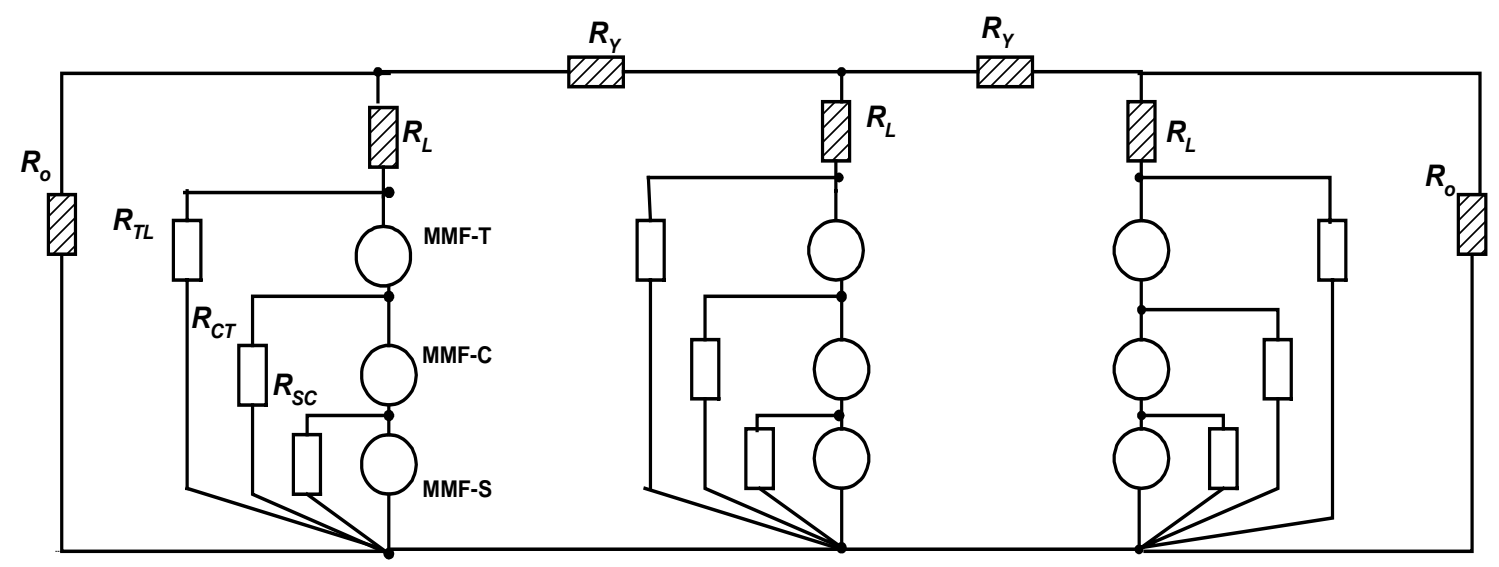

Figure 5.2 Magnetic Circuit for Five-legged Core Transformer 


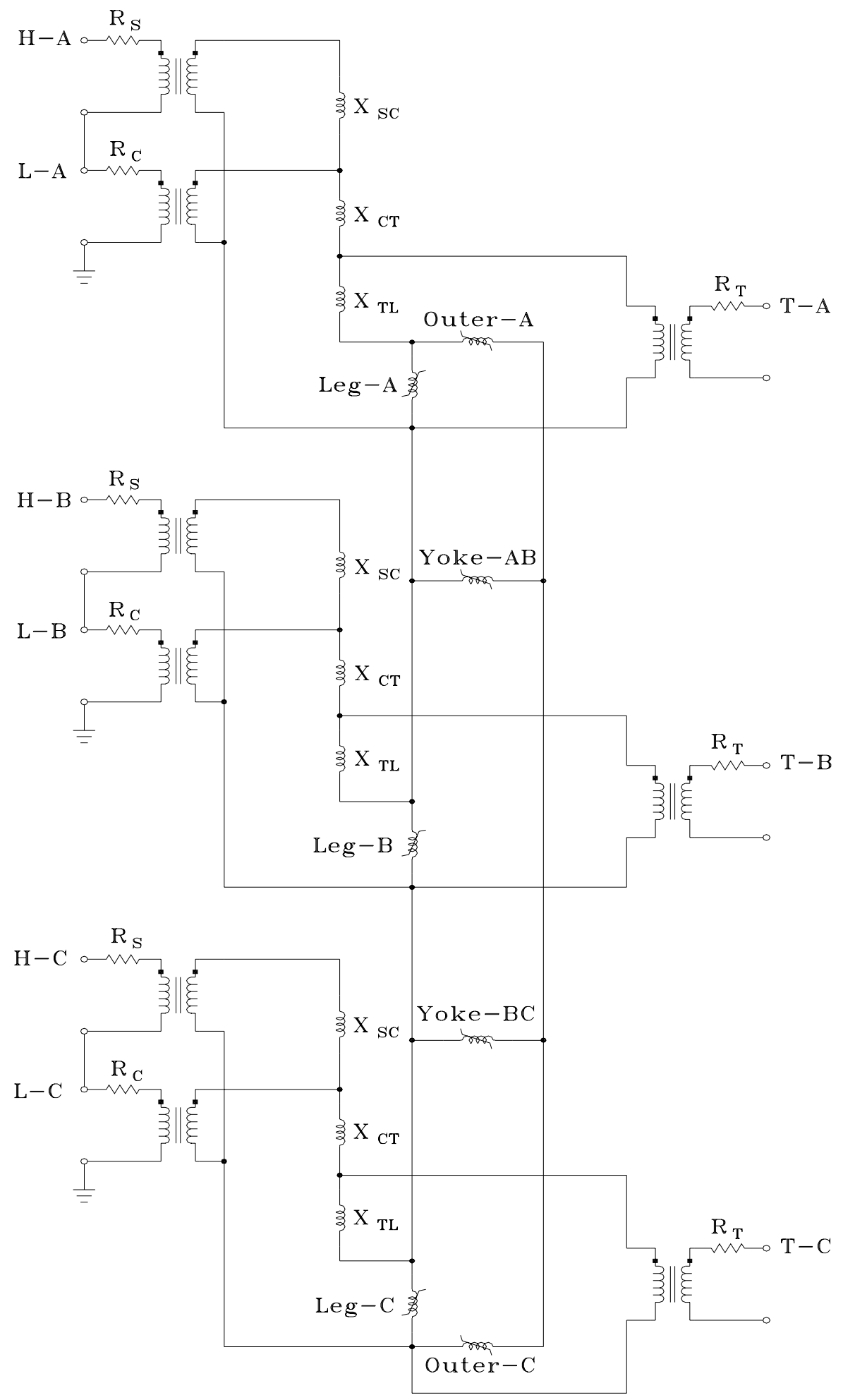

Figure 5.3 Equivalent Electric Circuit for Five-Legged Core Transformer 


\subsection{Three-Legged Core Transformer}

In three-phase three-legged core-form transformers, as shown in Figure 5.4, the positive-sequence flux has a zero sum at every instant and cancels out via the yoke. The zerosequence flux must find a return path outside the yoke. The tank's walls offer return paths to the leakage flux for zero sequence current. In Figure 5.5, " $\mathfrak{R}_{\mathrm{o}}$ " is called the zero sequence or homopolar path because this is the path through which the flux would flow if zero sequence voltage are applied to all three phases of the transformer. This path is basically through the insulating oil and tank surrounding the core and windings. Since most of this path has $\mu_{\mathrm{r}}=1.0$, the impedance of the zero sequence path is much smaller than the impedance of the core leg and core yoke. This lowers the magnitude of the zero-sequence impedance.

During the zero-sequence test, the delta on the tertiary voltage side should be opened up. If it is closed it would allow zero sequence currents to circulate in the delta, and in effect short out the zero sequence impedance.

To represent the zero-sequence flux path, a zero-sequence element may be placed in the middle leg's zero-sequence path of the electrical equivalent. In [47], simulations are performed with and without this element with no significant difference in results.

Figure 5.6 shows the resulting equivalent electric circuit for the three-legged core transformer. 


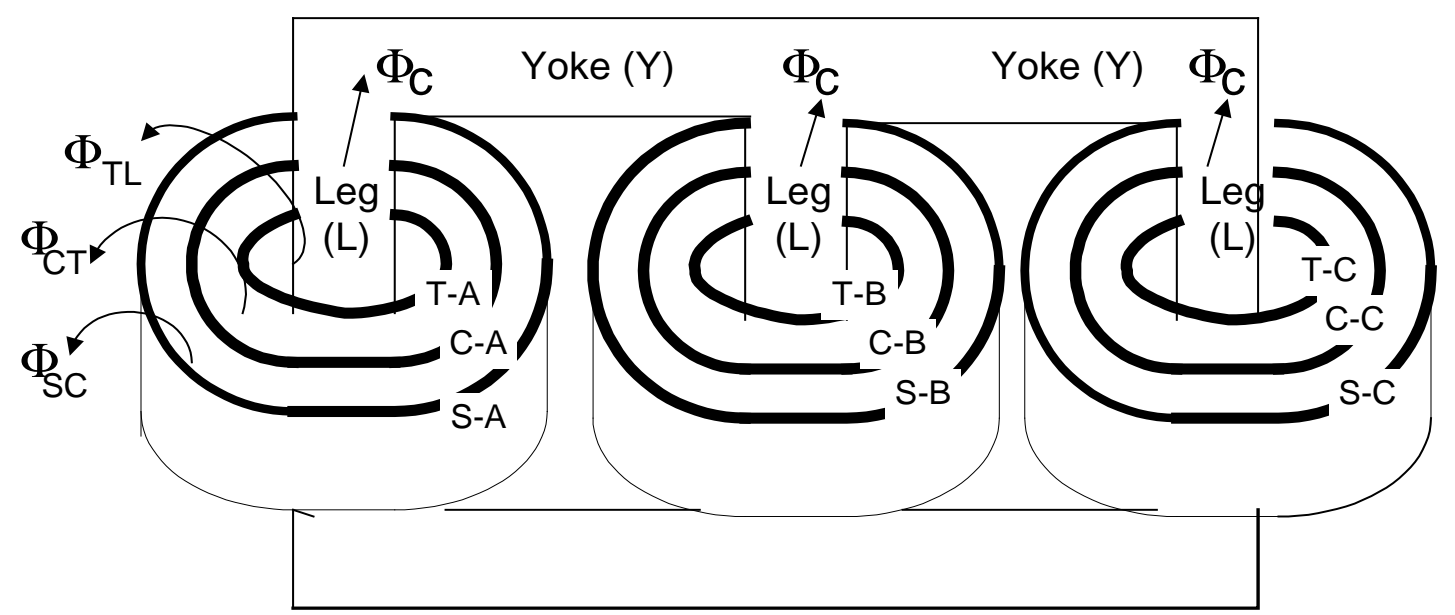

Figure 5.4 Three-legged Core Transformer Structure

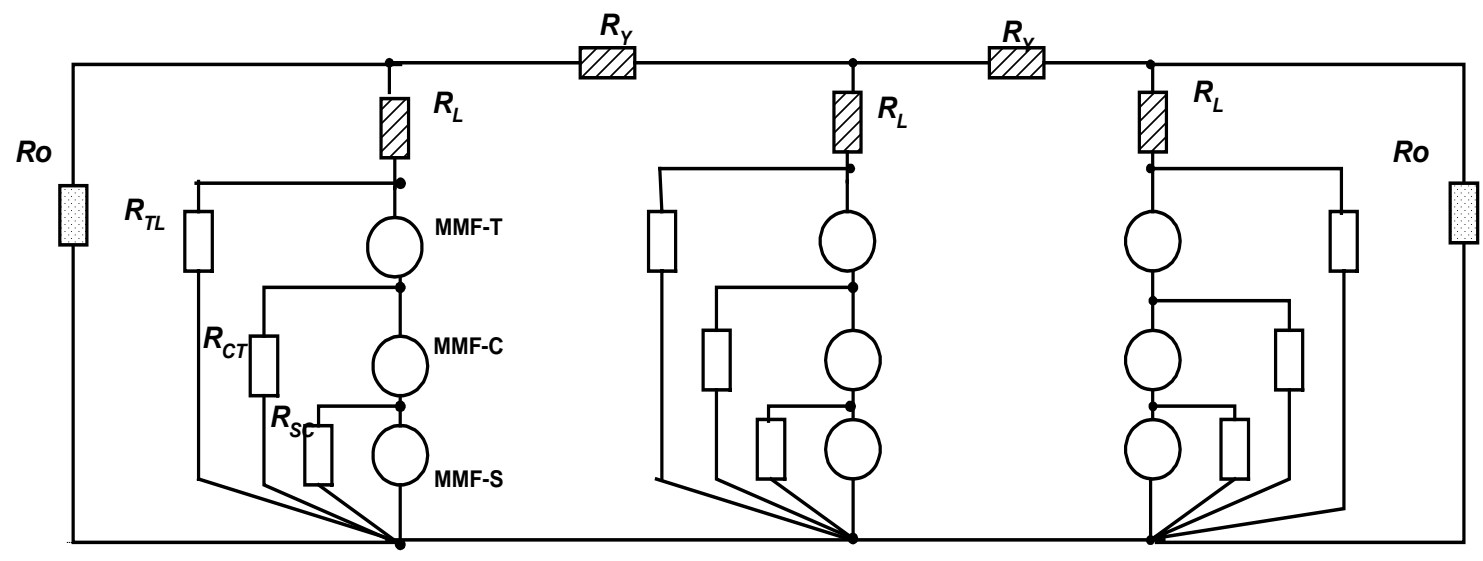

Figure 5.5 Magnetic Circuit for Three-legged Core Transformer 


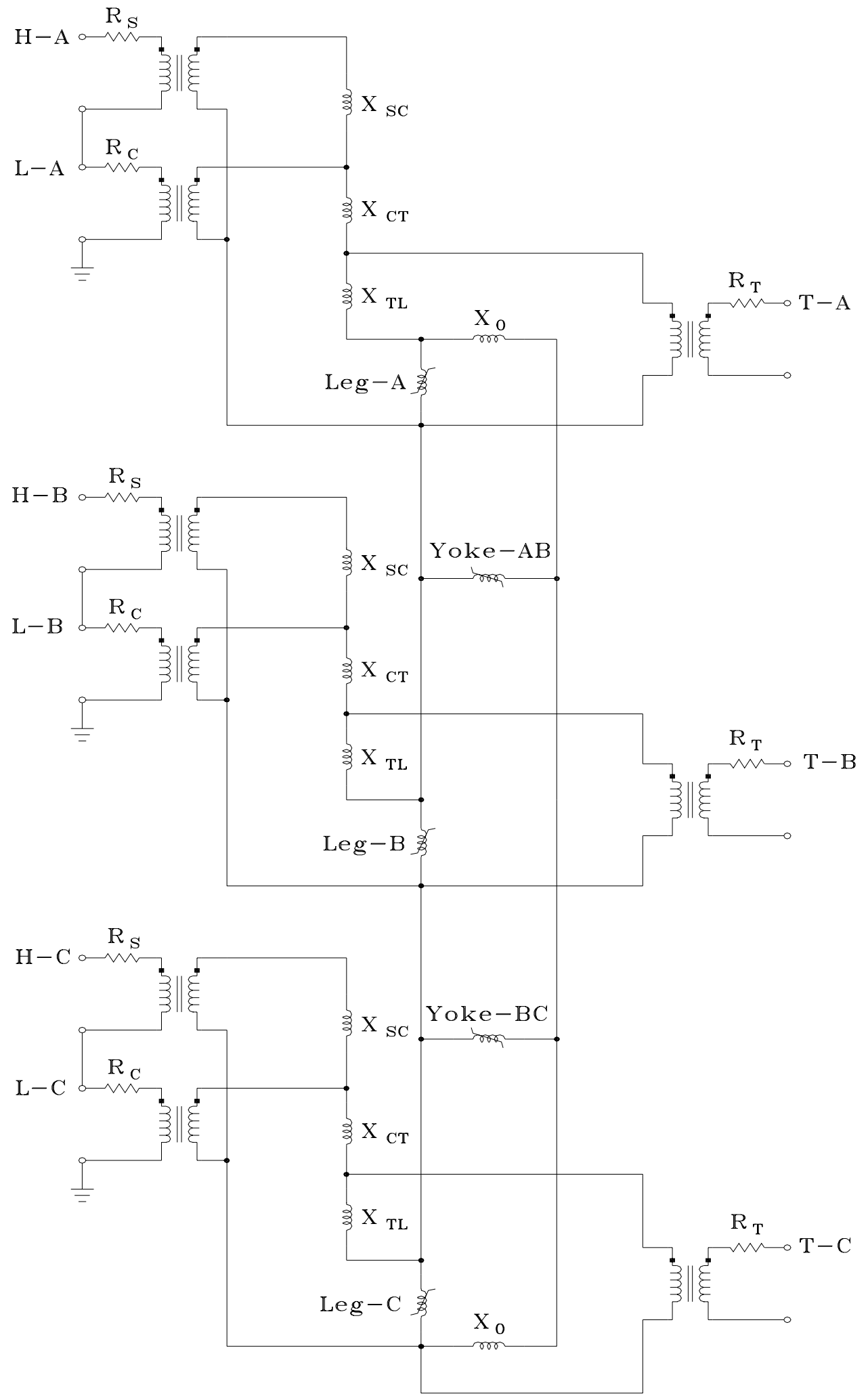

Figure 5.6 Equivalent Electric Circuit for Three-legged Core Transformer 


\subsection{Shell-form Transformer}

The structure of a three-phase shell-form transformer is shown in Figure 5.7. The fluxes in the core are $\Phi_{1}=\Phi_{A} / 2, \Phi_{2}=\Phi_{B} / 2, \Phi_{3}=\Phi_{C} / 2, \Phi_{4}=\Phi_{A}-\Phi_{B}, \Phi_{5}=\Phi_{B}-\Phi_{C}$,

The lumped magnetic circuit representing the three-phase shell-form transformer is shown in Figure 5.8. The windings are represented by MMF sources. The reluctances due to the flux through the iron core are represented by solid rectangles, whereas the reluctances due to leakage fluxes through the gaps between windings are represented by outlined rectangles.

Reluctances $\mathfrak{R}_{\mathrm{x}}, \mathfrak{R}_{\mathrm{o}}, \mathfrak{R}_{\mathrm{m}}$ represent the portions of the core with a cross section that is about $50 \%$ that of the core inside the windings. Reluctances $\mathfrak{R}_{\mathrm{x}}, \mathfrak{R}_{\mathrm{o}}, \mathfrak{R}_{\mathrm{m}}$ represent the parallel combinations of two reluctances for upper and lower core sections (the core structure in Figure 5.7 was horizontally folded due to symmetry, simplifying the resulting lumped magnetic circuit). These portions of the core thus have the same conditions of saturation as the core inside the windings.

A shell-form transformer is designed so that the middle limbs (" $\Re_{\mathrm{y}}$ ") can carry two fluxes, permitting economy in the core construction and lower losses. The mean turn length is usually longer than for a comparable core-form design, while the iron path is shorter.

Figure 5.9 shows the equivalent electric circuit for the shell-form transformer. 


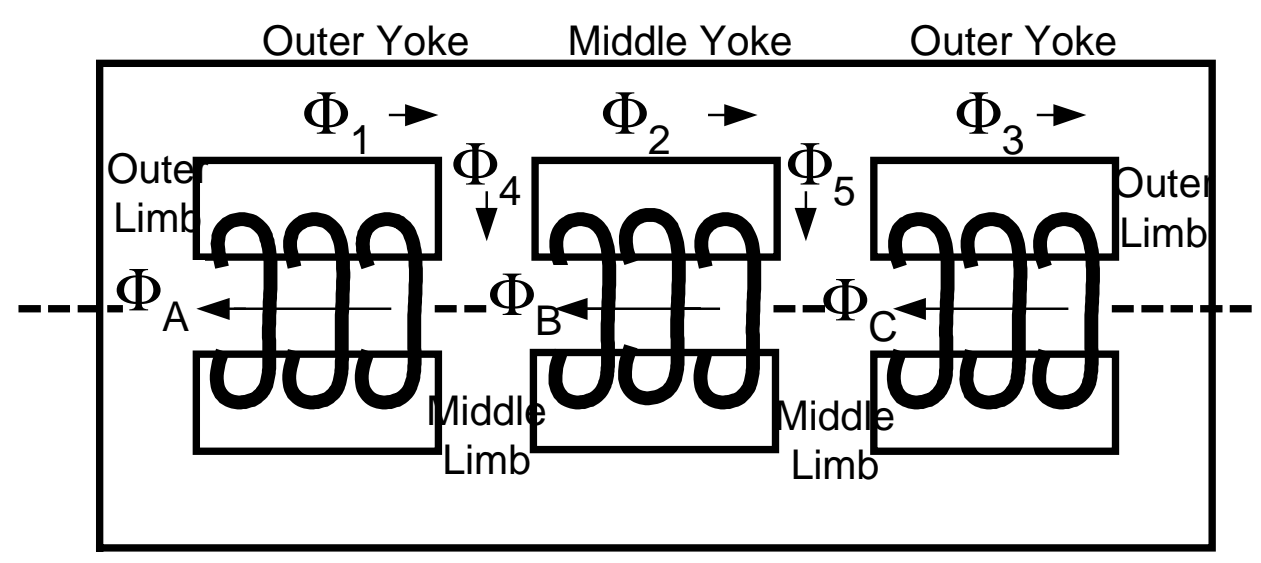

Figure 5.7 Shell-form Transformer Structure

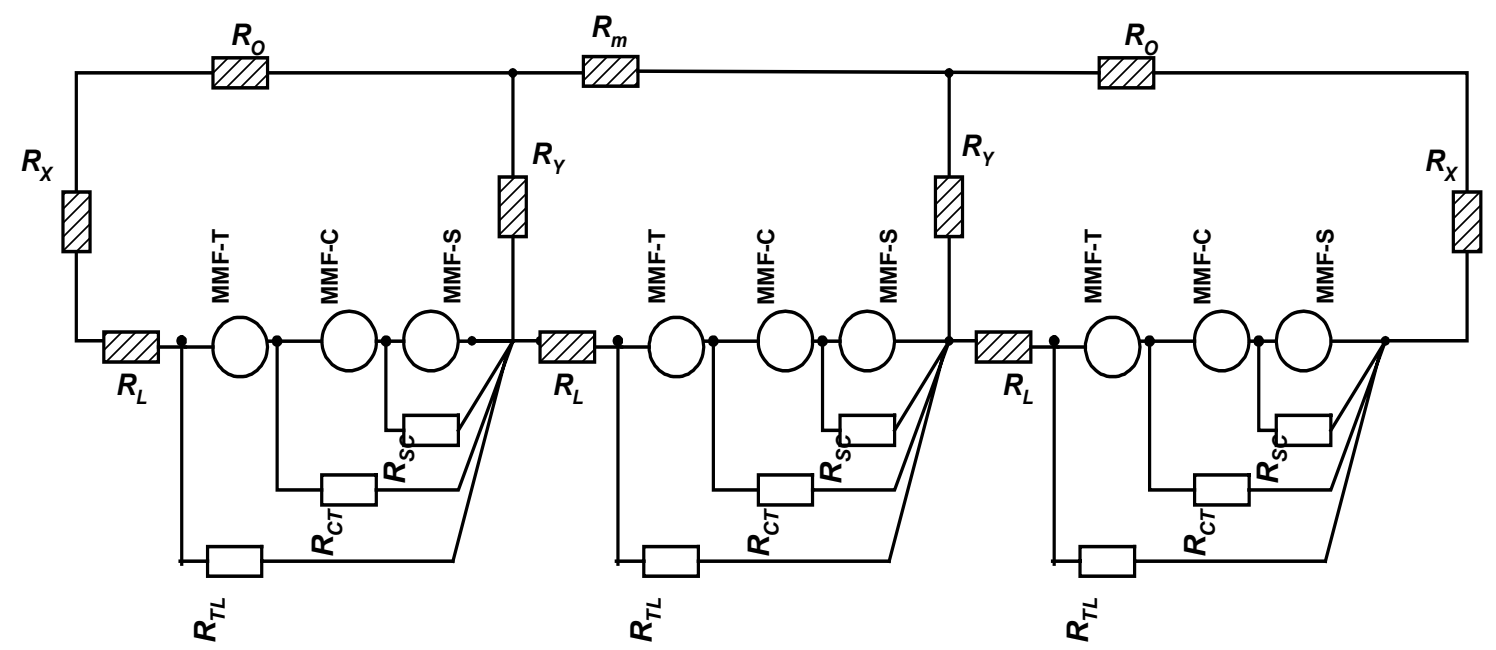

Figure 5.8 Magnetic Circuit for Shell-form Transformer 


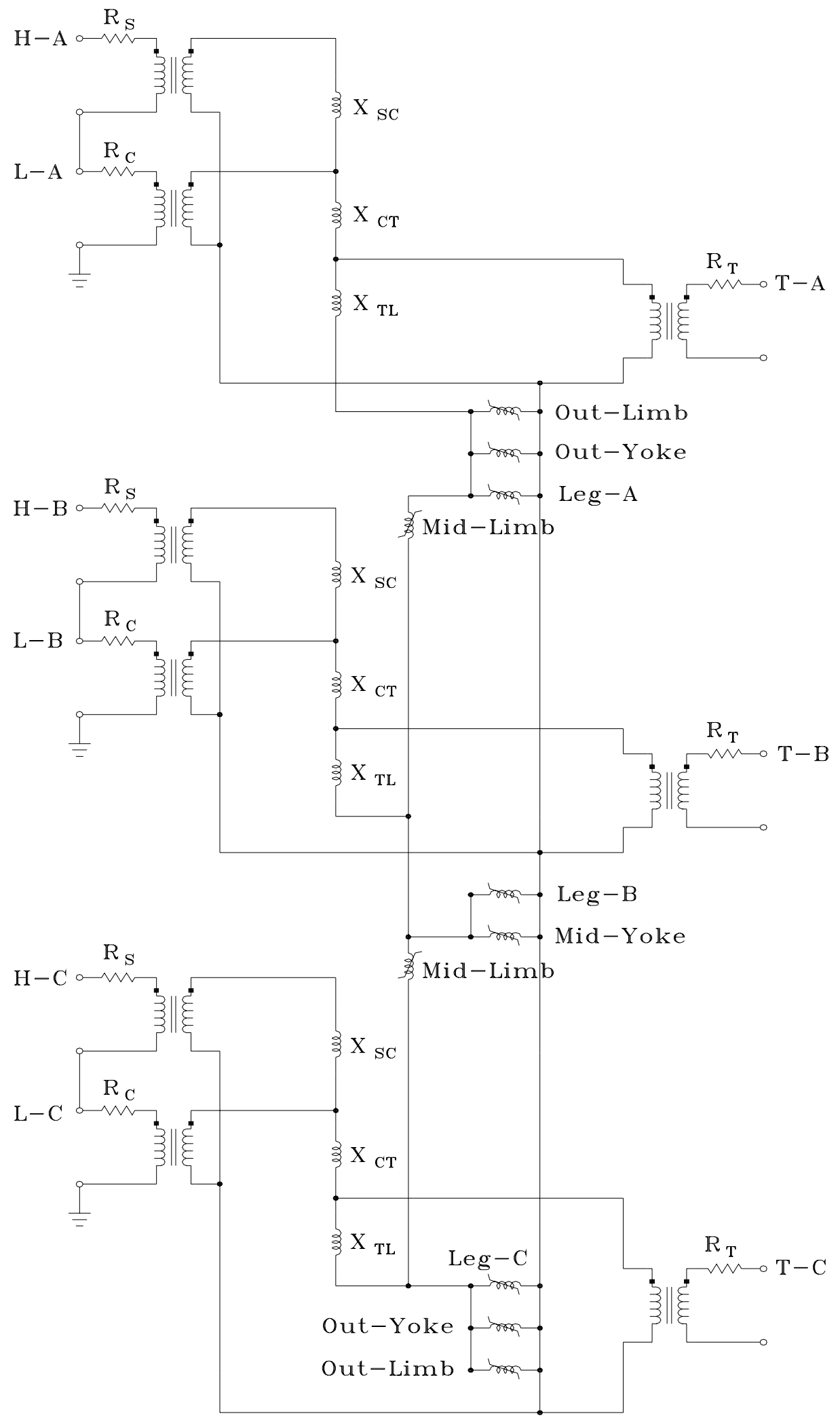

Figure 5.9 Equivalent Electric Circuit for Sell-form Transformer 


\section{CHAPTER 6 \\ PARAMETER ESTIMATION FOR TRANSFORMER MODELS}

This chapter presents the parameter estimation (leakage inductances, core saturation components, core loss components) for the duality-based equivalent circuit models of three-phase five-legged, three-phase three-legged and three-phase shell-form autotransformers that were developed in Chapter 5.

\subsection{The Five-Legged Core Transformer}

\subsubsection{Leakage Inductance Derivation}

Leakage inductance is due to flux linking one winding but not another. The flux that "leaks" typically passes through air or other nonmagnetic materials and may also find low-reluctance paths through the transformer tank and other metallic fittings. Estimation of the reluctance of the leakage path is done by estimating the distribution of leakage flux and the resulting flux linkage across the involved winding(s). This distribution depends on the geometric configuration of the coils. A detailed derivation of leakage reactance between two windings of equal axial lengths is given in [42].

Figure 6.1 shows the MMF functions related to the "binary" short-circuit leakage inductances, for each pair of windings. A cylindrically-wound three-winding transformer is assumed. Dimensions denoted by "a" are duct or insulation thickness and "b" is coil 
thickness. Coils labeled as T, C, and S stand for Tertiary, Common, and Series, respectively. L denotes the surface of the core leg.

Leakage reactances for the three-winding transformer in Figure 6.1 are represented as Equations (6.1) through (6.4) [42]. This derivation assumes linear flux distribution across the coil thickness. For each binary pair of coils, the MMF increases linearly across the inner winding, remains constant across the duct, then decreases linearly with radius through the outer winding.

One leakage flux, important for detailed models but not considered (or measurable) in factory tests, is the flux linked by the tertiary coil but not flowing in the core. This can be conceptually dealt with by assuming a fictitious infinitely-thin coil at the surface of the coil leg, L. The related MMF function is labeled as T-L.

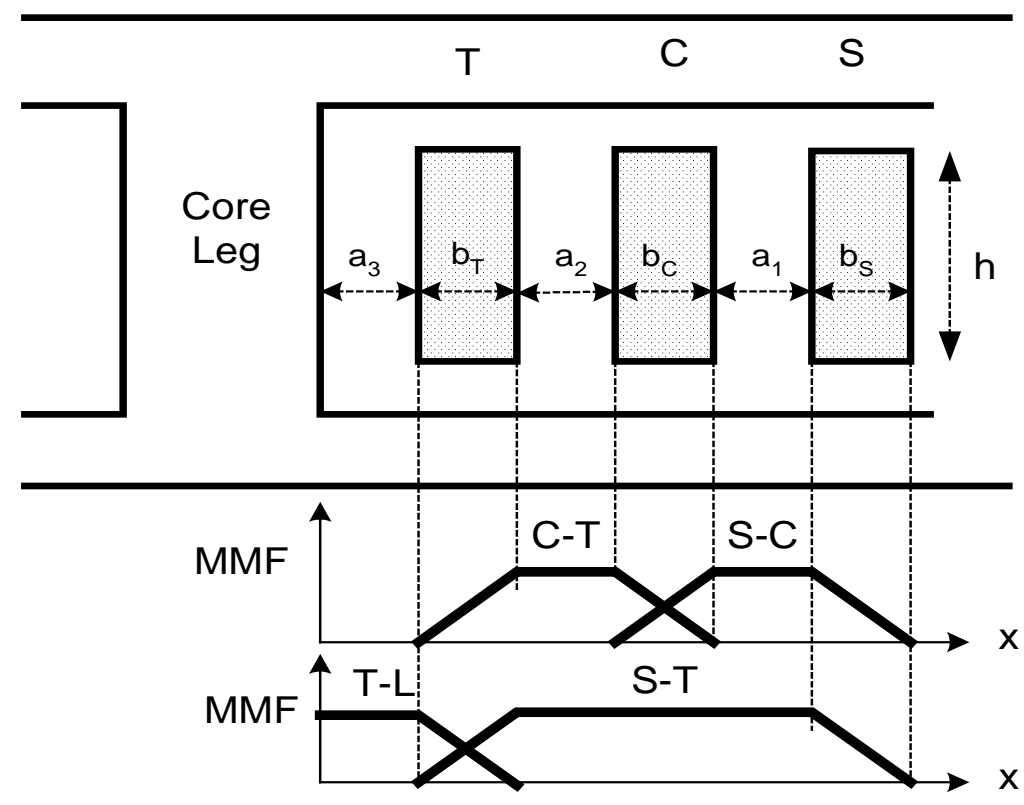

Figure 6.1 Transformer Cross Section with Three Windings and MMF Distributions 


$$
\begin{aligned}
& X_{S C}=\frac{\mu_{o} 2 \pi f N^{2} \cdot L m t \cdot\left(\frac{b_{C}+b_{S}}{3}+a_{1}\right)}{h} \\
& X_{C T}=\frac{\mu_{o} 2 \pi f N^{2} \cdot L m t \cdot\left(\frac{b_{C}+b_{T}}{3}+a_{2}\right)}{h} \\
& X_{S T}=\frac{\mu_{o} 2 \pi f N^{2} \cdot L m t \cdot\left(\frac{b_{S}+b_{T}}{3}+a_{1}+a_{2}+b_{C}\right)}{h} \\
& X_{T L}=\frac{\mu_{o} 2 \pi f N^{2} L m t \cdot\left(\frac{b_{T}}{3}+a_{3}\right)}{h}
\end{aligned}
$$

$\mu_{0}:$ Magnetic permeability of free space $b_{T}, b_{C}, b_{S}:$ Radial thickness of winding $h$ : Axial height of winding and duct circumference $a_{1}, a_{2}, a_{3}:$ Radial width of duct N: Number of winding turns Lmt: Mean turn length or

The electrical equivalent circuit for the resulting duality model is given in Figure 6.2 (adapted from [5]). The leakage inductances in Figure 6.2 are broken down into the components shown in Figure 6.3 to obtain implementable parameters. Transforming the 3 binary short-circuit reactances, in a star-equivalent representation is done by Equation (6.5) through (6.7).

$$
\begin{aligned}
& X_{S}=\frac{X_{S T}+X_{S C}-X_{C T}}{2} \\
& X_{C}=\frac{X_{C T}+X_{S C}-X_{T S}}{2} \\
& X_{T}=X_{T-1}+X_{T-2}=\frac{X_{C T}+X_{T S}-X_{S C}}{2}
\end{aligned}
$$




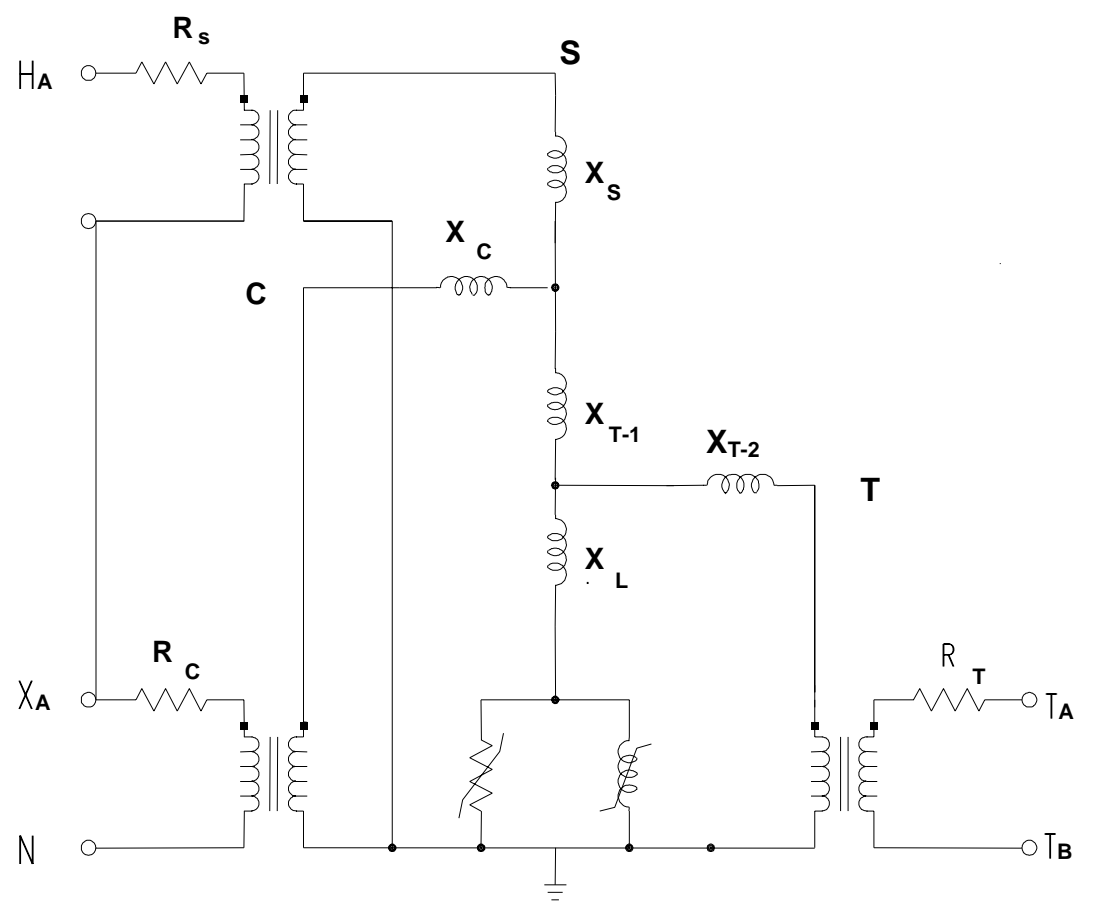

Figure 6.2 Electrical Equivalent Circuits for Leakage Reactance

The reactance labeled " $\mathrm{X}_{1}$ " in Figure 6.3 is the leakage reactance associated with the thickness of the series winding and the duct between the series winding and the common winding. Reactances " $\mathrm{X}_{2}$ " and " $\mathrm{X}_{\mathrm{C}}$ " are associated with common winding. Reactance " $\mathrm{X}_{2}$ " is $1.5 \times \mathrm{X}_{\mathrm{COM}}$ and inductance $" \mathrm{X}_{\mathrm{C}}$ " is $-0.5 \times \mathrm{X}_{\mathrm{COM}}$, where $\mathrm{X}_{\mathrm{COM}}$ is the portion of the leakage reactance due to the thickness of the common winding. In a similar manner, inductances " $\mathrm{X}_{4} "$ and $" \mathrm{X}_{\mathrm{T}-2}$ " represent the leakage reactance contribution of the tertiary winding. Reactance " $\mathrm{X}_{4}$ " is $1.5 \times \mathrm{X}_{\text {TER }}$ and inductance " $\mathrm{X}_{\mathrm{T}-2}$ " is $-0.5 \times \mathrm{X}_{\text {TER }}$, where $\mathrm{X}_{\mathrm{TER}}$ is the portion of the leakage reactance due to the thickness of the tertiary winding. Finally, " $\mathrm{X}_{3}$ " equals the leakage value calculated for the duct between the common winding and the tertiary winding, and " $\mathrm{X}_{5}$ " equals the leakage value calculated for the duct between the tertiary winding and the core. 


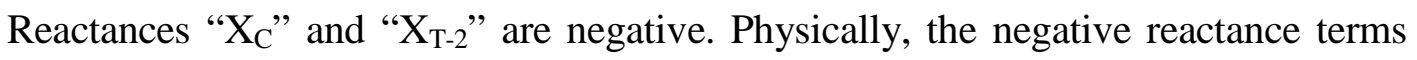
are a result of coil thickness as demonstrated by both the short-circuit test equations. Negative reactances are inserted in series with the winding resistances of the transformer model to compensate for coil thickness, with one just inside the inner winding and one just outside the outer winding. Leakage reactance values obtained from the energized winding thickness are one-third the reactance value contributed by the thickness of the same windings when they are un-energized.

$$
\begin{aligned}
& X_{C O M}=\frac{\mu_{o} 2 \pi f N^{2} \cdot \operatorname{Lmt} \cdot\left(\frac{b_{C}}{3}\right)}{h}, \quad X_{C}=\frac{-X_{C O M}}{2} \\
& X_{2}=1.5 \times X_{C O M} \\
& X_{T E R}=\frac{\mu_{o} 2 \pi f N^{2} \cdot \operatorname{Lmt} \cdot\left(\frac{b_{T}}{3}\right)}{h} \quad, X_{T-2}=\frac{-X_{T E R}}{2} \\
& X_{4}=1.5 \times X_{T E R} \\
& X_{1}=\frac{\mu_{o} 2 \pi f N^{2} \cdot \operatorname{Lmt} \cdot\left(b_{S} / 3+a_{1}\right)}{h} \\
& X_{3}=\frac{\mu_{o} 2 \pi f N^{2} \cdot \operatorname{Lmt} \cdot\left(a_{2}\right)}{h} \\
& X_{5}=\frac{\mu_{o} 2 \pi f N^{2} \cdot L m t \cdot\left(a_{3}\right)}{h}
\end{aligned}
$$




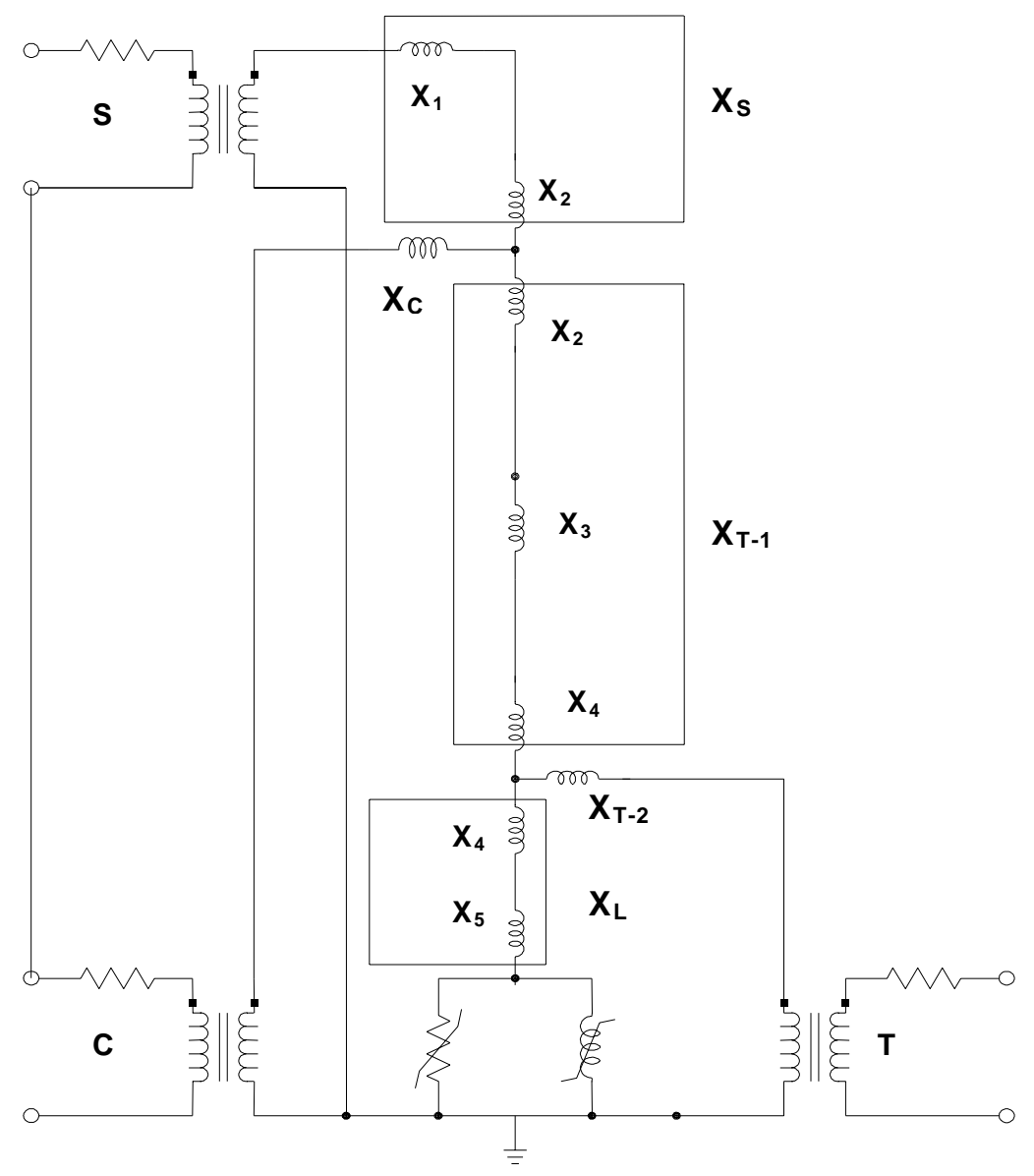

Figure 6.3 Electrical Equivalent Circuits with Breakdown of Leakage Effects

Reconciling the leakage inductances of Figure 6.3 with the MMF distributions of Figure 6.1, it is seen that: $X_{S C}=X_{1}+X_{2}+X_{C}, X_{C T}=X_{C}+X_{2}+X_{3}+X_{4}+X_{T-2}, X_{S T}=X_{1}+X_{2}+X_{2}$ $+X_{3}+X_{4}+X_{T-2}$ and $X_{T L}=X_{T-2}+X_{4}+X_{5}$ 


\subsubsection{Practical Implementation of Leakage inductance}

In the test reports, $\mathrm{X}_{\mathrm{HX}}, \mathrm{X}_{\mathrm{HY}}, \mathrm{X}_{\mathrm{XY}}$ are given as binary short-circuit impedances in per unit on the base of the terminal ratings. The autotransformer can be represented as a transformer with 3 windings $\mathrm{S}, \mathrm{C}$, and $\mathrm{T}$. The voltage ratings are $V_{S}=V_{H^{-}} V_{X}, V_{C}=V_{X}$, $V_{T}=V_{Y}$. Therefore, $\mathrm{X}_{\mathrm{C}}, \mathrm{X}_{\mathrm{S}}, \mathrm{X}_{\mathrm{T}}$ are calculated in the winding base [17] as:

$$
\begin{gathered}
N=\frac{V_{H}}{V_{X}}, X_{S C}=X_{H X}\left(\frac{N}{N-1}\right)^{2}, X_{C T}=X_{X Y} \\
X_{T S}=X_{H Y}\left(\frac{N}{N-1}\right)-X_{X Y}\left(\frac{1}{N-1}\right)+X_{H X}\left(\frac{N}{(N-1)^{2}}\right) \\
X_{T}=\frac{X_{C T}+X_{T S}-X_{S C}}{2}, X_{C}=\frac{X_{C T}+X_{S C}-X_{T S}}{2}, X_{S}=\frac{X_{T S}+X_{S C}-X_{C T}}{2}
\end{gathered}
$$

In case of the example transformer,

$$
\begin{aligned}
& X_{S C}=.27686, X_{C T}=0.81259, X_{S T}=1.3121(\mathrm{ohms}) \text { at the } 13.8-\mathrm{kV} \text { base } \\
& X_{S}=0.38819, X_{C}=-.11133, X_{T}=0.92391(\mathrm{ohms}) \text { at the } 13.8-\mathrm{kV} \text { base }
\end{aligned}
$$

This is consistent with the development for leakage inductance presented in the previous section. The simplified short-circuit equivalent circuit is shown in Figure 6.4. $X_{C}$ for the common winding is typically a negative inductance. However, at this level of detail, the corresponding equivalent circuit would inadequately describe all the leakages related to short-circuit behaviors of the transformer (see Section 3.1).

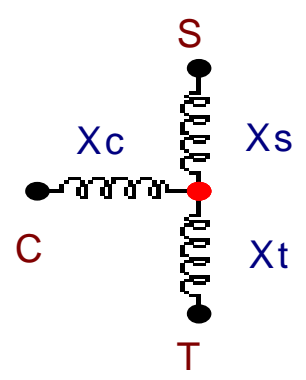

Figure 6.4 Three-winding Equivalent Circuit from Test Report 
Table 6.1 gives an example for normalized winding thickness calculated from DC resistance of coils. " $\mathrm{b}_{\mathrm{T}}$ " and " $\mathrm{a}_{2}$ " are smaller than " $\mathrm{b}_{\mathrm{S}}$ " and " $\mathrm{a}_{1}$ ". Therefore, $\mathrm{X}_{\mathrm{CT}}$ from Equation (6.2) should be smaller than $\mathrm{X}_{\mathrm{SC}}$ from Equation (6.1). However, $\mathrm{X}_{\mathrm{CT}}$ is larger than $\mathrm{X}_{\mathrm{SC}}$ for most test data. In this case, the common winding and series winding need to be separated as shown in Figure 6.5.

Table 6.1 Normalized Winding Thickness Based on Coil DC Resistances

\begin{tabular}{|c|c|c|c|}
\hline & Winding- $\mathrm{T}$ & Winding-C & Winding-S \\
\hline Voltage Ratio & 13.8 & 68.1 & 131.1 \\
\hline Turns Ratio (1) & 1 & 4.93 & 9.5 \\
\hline Normalized Air Gap Thickness * & $1\left(\mathrm{a}_{3}\right)$ & $4.93\left(\mathrm{a}_{2}\right)$ & $14.43\left(\mathrm{a}_{1}\right)$ \\
\hline $\mathrm{R}_{\mathrm{DC}}(\mathrm{ohm})$ & 0.0175 & 0.0545 & 0.2098 \\
\hline $\mathrm{R}_{\mathrm{DC}}(\mathrm{ratio})(2)$ & 1 & 3.11 & 11.99 \\
\hline Conductor Size $(\mathrm{pu})=(1) /(2)$ & 1 & 1.59 & 1.20 \\
\hline Winding Area $(\mathrm{pu})=(1)^{2} /(2)$ & 1 & 7.82 & 7.53 \\
\hline Normalized Winding Thickness & $1\left(\mathrm{~b}_{\mathrm{T}}\right)$ & $7.82\left(\mathrm{~b}_{\mathrm{C}}\right)$ & $7.53\left(\mathrm{~b}_{\mathrm{S}}\right)$ \\
\hline
\end{tabular}

*: Air gap thickness is generally proportional to winding voltage.

Thus, normalized air gap thickness is assumed the same as the turns ratio.

Equations (6.17) through (6.19) demonstrate leakage inductances based on the physical dimensions of Figure 6.5. These equations have five unknowns. For the model of Figure 6.2, parameter estimations and some assumptions are necessary. The summation of the model parameters should be identical to the given short-circuit test data.

$$
\begin{aligned}
& X_{S C}=\frac{\mu_{o} 2 \pi f N^{2} \cdot \operatorname{Lmt} \cdot \frac{1}{2} \cdot\left(\frac{b_{C}+b_{S}}{6}+a_{1}\right)}{h} \\
& X_{C T}=\frac{\mu_{o} 2 \pi f N^{2} \cdot \operatorname{Lmt} \cdot\left(\frac{b_{C}+b_{T}}{3}+a_{2}+\frac{a_{1}}{2}+\frac{b_{S}}{8}\right)}{h}
\end{aligned}
$$




$$
X_{S T}=\frac{\mu_{o} 2 \pi f N^{2} \cdot L m t \cdot\left(\frac{b_{S}+b_{T}}{3}+a_{2}+\frac{3 \cdot a_{1}}{2}+\frac{5 \cdot b c}{8}\right)}{h}
$$

From Table 6.1, the ratios for winding width or winding area can be assumed from $R_{\mathrm{DC}}$ and the ratios between $b_{\mathrm{S}}, \mathrm{b}_{\mathrm{C}}$ and $\mathrm{b}_{\mathrm{T}}$ can be assumed as $\mathrm{b}_{\mathrm{S}}=7.53 \cdot \mathrm{b}_{\mathrm{T}}$, and $\mathrm{b}_{\mathrm{C}}=7.82 \cdot \mathrm{b}_{\mathrm{T}}$. From voltage ratio, ratios for air gaps $a_{1}, a_{2}$ and $a_{3}$ can be assumed as $a_{1}=14.43 \cdot a_{3}$, $a_{2}=4.93 \cdot a_{3}$. Now, there are two unknowns for Equations (6.17) through (6.19). The least square fitting technique gives two values which minimize the differences in the thicknesses of coils and air gaps, giving approximately $b_{C}=1.5653, b_{T}=0.2002, b_{S}=1.5073$ and $\mathrm{a}_{1}=0.0417, \mathrm{a}_{2}=0.0149, \mathrm{a} 3=0.003$. From these and Equation (6.8) through (6.14), the necessary reactance in Figures 6.2 and 6.3 can be obtained. Table 6.2 represents the breakdown of coil and duct components of each binary short-circuit reactance, corresponding to Figures 6.1 through 6.3. 


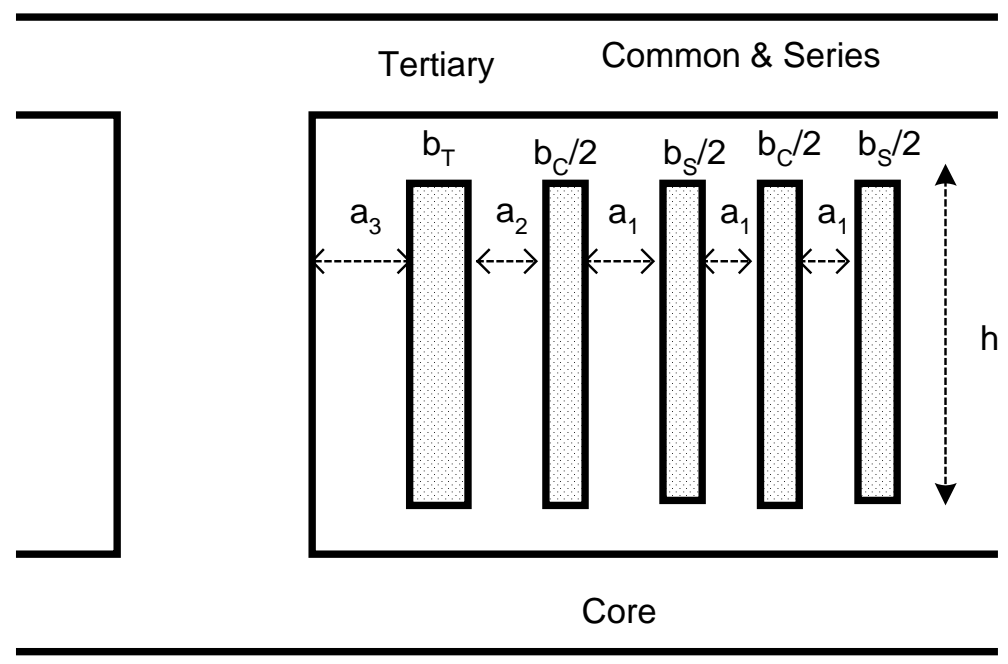

Figure 6.5 Cross-Section with Main Leakage Paths for Concentric Windings

Table 6.2 Calculated Leakage Reactance in Ohms.

\begin{tabular}{|c|c|c|c|c|c|}
\hline & $\mathrm{S}-\mathrm{C}$ & $\mathrm{C}-\mathrm{T}$ & $\mathrm{S}-\mathrm{T}$ & \multicolumn{2}{|c|}{ T-Core } \\
\hline Series $(\mathrm{S})$ & \multirow{2}{*}{$\begin{array}{c}0.3882 \\
\left(\mathrm{X}_{\mathrm{S}}\right)\end{array}$} & & \multirow{2}{*}{$\begin{array}{c}0.3882 \\
\left(X_{S}\right)\end{array}$} & & \\
\hline $\operatorname{Duct}\left(\mathrm{a}_{1}\right)$ & & & & & \\
\hline Common $(\mathrm{C})$ & $\begin{array}{c}-0.1113 \\
\left(X_{C}\right)\end{array}$ & $\begin{array}{c}-0.1113 \\
\left(X_{C}\right)\end{array}$ & & & \\
\hline $\operatorname{Duct}\left(\mathrm{a}_{2}\right)$ & & $\begin{array}{c}0.9573 \\
\left(\mathrm{X}_{\mathrm{T}-1}\right)\end{array}$ & $\begin{array}{c}0.9573 \\
\left(\mathrm{X}_{\mathrm{T}-1}\right)\end{array}$ & & \\
\hline \multirow[t]{2}{*}{ Tertiary (T) } & & $\begin{array}{c}-0.0334 \\
\left(\mathrm{X}_{\mathrm{T}-2}\right)\end{array}$ & $\begin{array}{c}-0.0334 \\
\left(\mathrm{X}_{\mathrm{T}-2}\right)\end{array}$ & $\begin{array}{r}-0.0 \\
(X\end{array}$ & \\
\hline & & & & $\begin{array}{c}0.1001 \\
\left(\mathrm{X}_{4}\right)\end{array}$ & \multirow{2}{*}{$\begin{array}{c}0.103 \\
\left(\mathrm{X}_{\mathrm{L}}\right)\end{array}$} \\
\hline $\operatorname{Duct}\left(\mathrm{a}_{3}\right)$ & & & & $\begin{array}{c}0.003 \\
\left(X_{5}\right)\end{array}$ & \\
\hline Total & $\begin{array}{c}0.2769 \\
\left(\mathrm{X}_{\mathrm{SC}}\right)\end{array}$ & $\begin{array}{c}0.8126 \\
\left(\mathrm{X}_{\mathrm{CT}}\right) \\
\end{array}$ & $\begin{array}{c}1.3121 \\
\left(\mathrm{X}_{\mathrm{ST}}\right) \\
\end{array}$ & \multicolumn{2}{|c|}{$\begin{array}{c}0.0696 \\
\left(\mathrm{X}_{\mathrm{TL}}\right) \\
\end{array}$} \\
\hline Test Report & 0.27686 & 0.81259 & 1.3121 & $\mathrm{~N}$ & \\
\hline
\end{tabular}




\subsubsection{Core Saturation Model}

Core dimensions, if available, can be used to calculate the saturation model for each core section. However, the dimensions of limbs and yokes are typically unknown. Instead of the exact dimensions of the core, the normalized ratios of core dimensions can be used. If the core dimension ratios are unknown, they must be assumed. In these cases, typical ratios can be used without great error, since the core dimension ratios vary within a small range $[9,19]$.

As explained earlier, five-legged core transformers are used in applications where lower transformer height is required. Therefore, the area of the yoke may be smaller than the area of leg. In this work, the core dimensions for a five-legged transformer are assumed as in Figure 6.6. Areas $\mathrm{A}_{6}$ and $\mathrm{A}_{7}$ were assumed to be the same as the center legs' area. In practice they can be as small as $0.5 \sim 0.7$ [4].

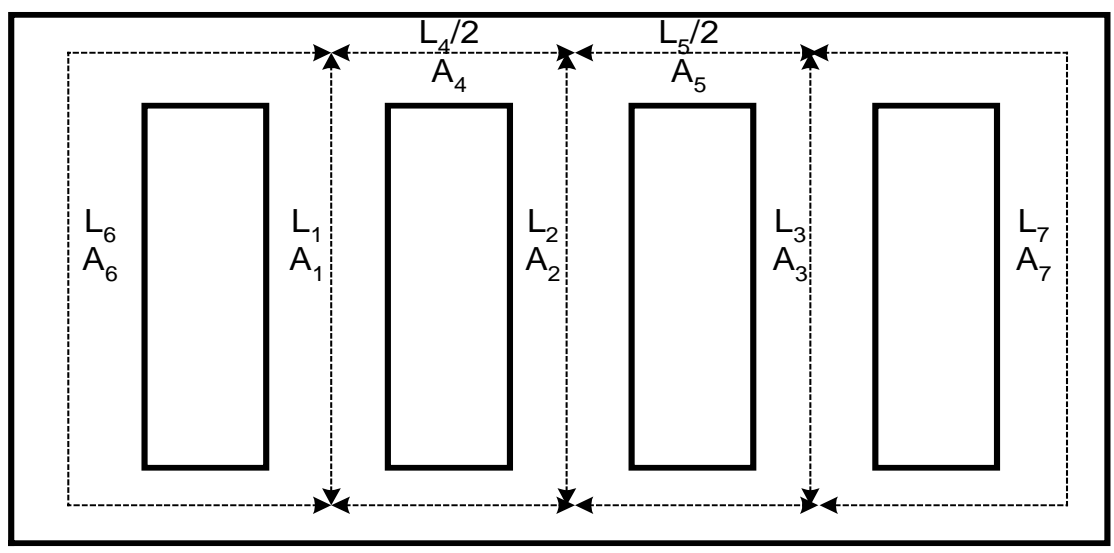

\begin{tabular}{|c|c|c|c|}
\hline \multicolumn{2}{|c|}{ Area ratio $\left(A_{1}=A_{2}=A_{3}=1\right)$} & \multicolumn{2}{c|}{ Length ratio $\left(L_{1}=L_{2}=L_{3}=1\right)$} \\
\hline Yoke $A_{4}=A_{5}$ & Outer $A_{6}=A_{7}$ & Yoke $L_{4}=L_{5}$ & Outer $L_{6}=L_{7}$ \\
\hline 1.0 & 1.0 & 1.725 & 2.21 \\
\hline
\end{tabular}

Notation: A: Area, L: Length, 1: Leg-A, 2:Leg-B, 3: Leg-C, 4:Yoke-AB, 5: Yoke-BC, 6: Outer-A,7: Outer-C

Figure 6.6 Dimension of Five-Leg Core Type Transformer 
The lumped parameter equivalent of Figure 6.6 is given by Figure 6.7. Note that all of the reluctances here are saturable. Only one set of MMFs (windings) is included, typical of no-load excitation. Fluxes are defined such that $\Phi_{1}, \Phi_{2}$, and $\Phi_{3}$ are the fluxes in the 3 center legs. Equations (6.20) through (6.23) follow, based on Ampere's circuital law and a normalized number of turns.

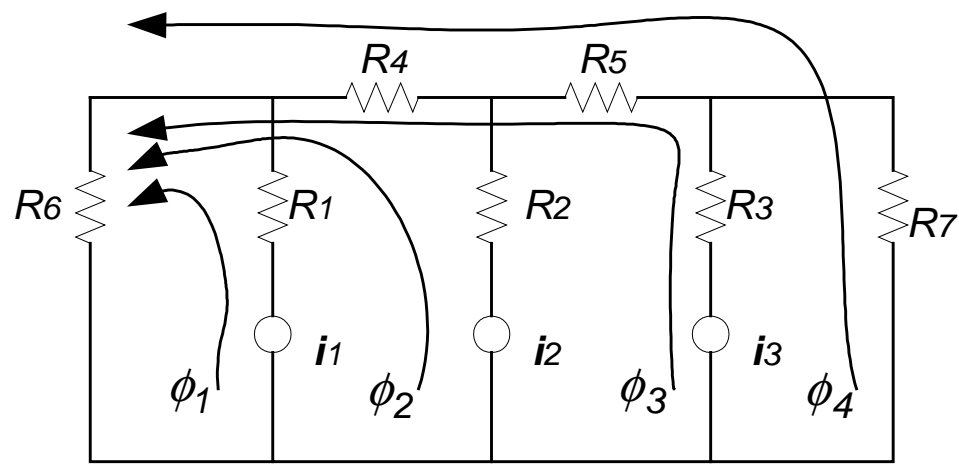

Figure 6.7 Magnetic Equivalent Circuit for Five-Legged Transformer

$$
\begin{aligned}
& i_{1}=\phi_{1} R_{1}+R_{6}\left(\phi_{1}+\phi_{2}+\phi_{3}+\phi_{4}\right) \\
& i_{2}=\phi_{2} R_{2}+R_{4}\left(\phi_{2}+\phi_{3}+\phi_{4}\right)+R_{6}\left(\phi_{1}+\phi_{2}+\phi_{3}+\phi_{4}\right) \\
& i_{3}=\phi_{3} R_{3}+R_{5}\left(\phi_{3}+\phi_{4}\right)+R_{4}\left(\phi_{2}+\phi_{3}+\phi_{4}\right)+R_{6}\left(\phi_{1}+\phi_{2}+\phi_{3}+\phi_{4}\right) \\
& i_{4}=0=\phi_{4} R_{7}+R_{5}\left(\phi_{3}+\phi_{4}\right)+R_{4}\left(\phi_{2}+\phi_{3}+\phi_{4}\right)+R_{6}\left(\phi_{1}+\phi_{2}+\phi_{3}+\phi_{4}\right)
\end{aligned}
$$

Where,

$$
\begin{array}{ll}
R_{1}=L_{1} /\left(\mu_{1} A_{1}\right) & \mu_{1}=\phi_{1} /\left(A_{1} H_{1}\right)=f\left(\phi_{1} / A_{1}\right) \\
R_{2}=L_{2} /\left(\mu_{2} A_{2}\right) & \mu_{2}=\phi_{2} /\left(A_{2} H_{2}\right)=f\left(\phi_{2} / A_{2}\right) \\
R_{3}=L_{3} /\left(\mu_{3} A_{3}\right) & \mu_{3}=\phi_{3} /\left(A_{3} H_{3}\right)=f\left(\phi_{3} / A_{3}\right) \\
R_{4}=L_{4} /\left(\mu_{4} A_{4}\right) & \mu_{4}=\left(\phi_{2}+\phi_{3}+\phi_{4}\right) /\left(A_{4} H_{4}\right)=f\left(\left(\phi_{2}+\phi_{3}+\phi_{4}\right) / A_{4}\right) \\
R_{5}=L_{5} /\left(\mu_{5} A_{5}\right) & \mu_{5}=\left(\phi_{3}+\phi_{4}\right) /\left(A_{5} H_{5}\right)=f\left(\left(\phi_{3}+\phi_{4}\right) / A_{5}\right) \\
R_{6}=L_{6} /\left(\mu_{6} A_{6}\right) & \mu_{6}=\left(\phi_{1}+\phi_{2}+\phi_{3}+\phi_{4}\right) /\left(A_{6} H_{6}\right)=f\left(\left(\phi_{1}+\phi_{2}+\phi_{3}+\phi_{4}\right) / A_{6}\right) \\
R_{7}=L_{7} /\left(\mu_{7} A_{7}\right) & \mu_{7}=\phi_{7} /\left(A_{7} H_{7}\right)=f\left(\phi_{4} / A_{7}\right)
\end{array}
$$


In order to estimate the core dimensions, variables are classified as known and unknown.

Known values: $\phi_{1}=v_{1} /(\omega N), \phi_{2}=v_{2} /(\omega N), \phi_{3}=v_{3} /(\omega N)$ $v=$ peak-voltage for each phase, $\omega=2 \pi f, N=$ number of turn

Magnetizing Current: $I_{A V G, R M S}=\left(I_{1, R M S}+I_{2, R M S}+I_{3, R M S}\right) / 3$

Note: Real component of $I_{E X}$ has been removed, as explained in Section 4.2 Core dimensions or normalized ratios

Unknown values: $\phi_{4}, a, b$ for $\mu=\frac{B}{H}=\frac{(1-b \cdot B)}{a}$

If the exact core dimensions, B-H curve, and winding turns are known, it is possible to calculate $\phi_{4}$ from Equations (6.20) through (6.23) by an iterative method. From the B-H curve and core dimensions, the saturation curve $(\lambda-i)$ for each core section can be derived. In most cases, the nonlinear curve for B-H is not known. Therefore, if only the core dimension ratios and average of the three RMS magnetizing currents at $100 \%$ and $110 \%$ voltages are given, an optimization techinique should make it possible to estimate the "a" and "b" coeffienents for the B-H Frolich equation and $\phi_{4}$ from Equation (6.20) through (6.23).

At this point, a MATLAB ${ }^{\circledR}$ program (Appendix B.1) was written to simulate the magnetizing current waveforms for a given set of paramters and to calculate the average RMS magnetizing current. Note that magnetic saturation is implemented in terms of a normalized B-H characteristic.

An iteratve method for $\phi_{4}$ was added into the MATLAB ${ }^{\circledR}$ program (Appendix B.2). If the calculated average current does not match the known value, the iteration continues 
to adjust the B-H curve. Optimization techniques give more accurate and faster solutions.

For all cases, forty points per half cycle are used for the RMS current calculation, since the waveforms are not sinusoidal.

The optimization performed is explained as follows: Details for waveform $i_{1}$ are given. Waveforms for $i_{2}$ and $i_{3}$ are obtained in a similar fashion based on Equations (6.21) and (6.22).

Minimize $f(a, b)=\left[\text { Measured } I_{A V G, R M S} @ 100 \% V \text {-Calculated } I_{A V G, R M S} @ 100 \% V\right]^{2}$

$+\left[\text { Measured } I_{A V G, R M S} @ 110 \% \text {-Calculated } I_{A V G, R M S} @ 110 \%\right]^{2}$

Subject to inequality constraints $0<a$ and $0<b<1$

Where, $\phi_{1}(k)=v_{1} /(\omega N) \times \sin (\pi \cdot k / 40)$ and $\phi_{4}(k)$ from each iteration After each iteration, $\phi_{4} R_{7}+R_{50}\left(\phi_{3}+\phi_{4}\right)+R_{4}\left(\phi_{2}+\phi_{3}+\phi_{4}+R_{6}\left(\phi_{1}+\phi_{2}+\phi_{3}+\phi_{4}\right)\right.$ should be zero according to Equation (6.23)

$$
\begin{aligned}
& B_{1}(k)=\phi_{1}(k) / A_{1} \quad \text { and } B_{6}(k)=\phi_{4}(k) / A_{6} \\
& \mu_{1}(k)=\frac{1-b \cdot B_{1}(k)}{a} \text { and } \mu_{6}(k)=\frac{1-b \cdot B_{6}(k)}{a} \\
& R_{1}(k)=L_{1} /\left(\mu_{1}(k) \cdot A_{1}\right) \quad \text { and } \quad R_{6}(k)=L_{6} /\left(\mu_{6}(k) \cdot A_{6}\right) \\
& i_{1}(k)=\phi_{1}(k) \cdot R_{1}(k)+R_{6}(k) \cdot \phi_{4}(k)
\end{aligned}
$$

$$
\begin{gathered}
I_{1, R M S}=\sqrt{\frac{\sum_{k=1}^{40}\left(i_{1}(k)\right)^{2}}{40}} \\
I_{R M S, A V G}=\left(I_{1, R M S}+I_{2, R M S}+I_{3, R M S}\right) / 3
\end{gathered}
$$

If core dimension ratios are assumed, but only the average magnetizing current at $100 \%$ voltage is known, it is necessary to know or assume the type of magnetic core material and then to use optimization tools to get the scaling factors described in Section 4.3 and $\phi_{4}$ 


\section{Results of MATLAB Simulations}

Using the optimization technique Fmincon, the results are $a=8.9379$ and $b=$ 0.5714 for the B-H equation. Figure 6.8 shows the B-H curve. The calculated RMS currents for three phases are $\left[\begin{array}{lll}109.2177 & 102.3031 & 102.3032\end{array}\right] \mathrm{A}$ at $100 \%$ voltage. The calculated average rms current is 104.61 and the difference from test report is $0.412 \mathrm{~A}$. The calculated RMS phases currents are $\left[\begin{array}{llll}229.3830 & 221.6717 & 221.6718\end{array}\right] \mathrm{A}$ at $110 \%$ voltage. The calculated average RMS current is $224.24 \mathrm{~A}$ and the difference between test report is $0.13 \mathrm{~A}$.

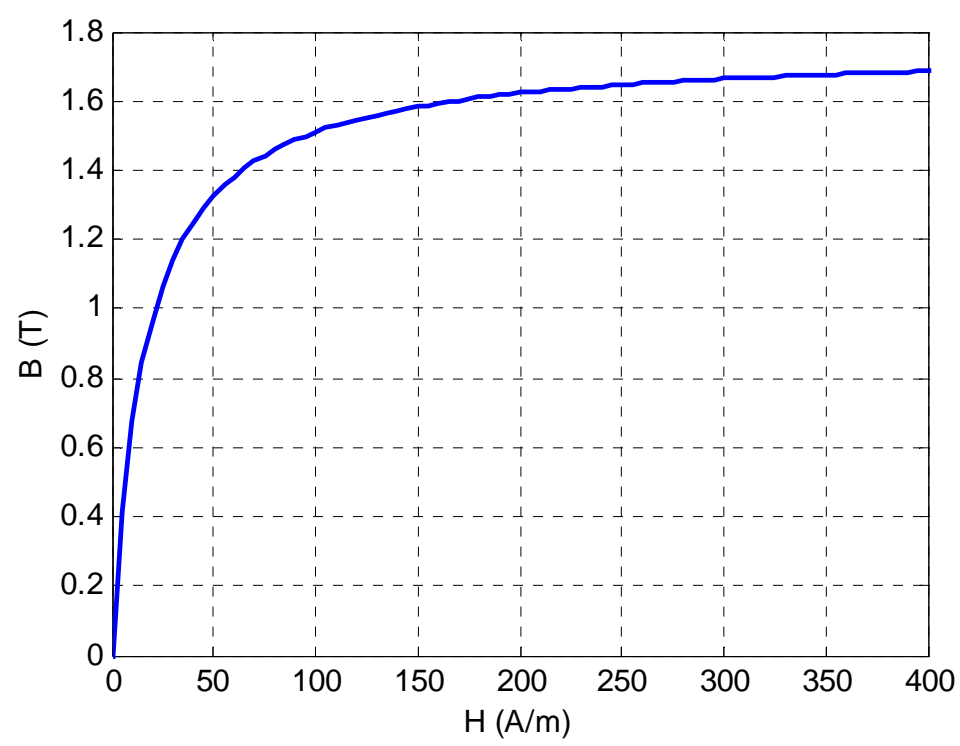

Figure 6.8 B-H Curve for Each Section

The magnetizing curves have the magnetic induction ( $\mathrm{B}$ in Tesla) on the vertical axis and magnetizing force $(\mathrm{H}$ in $\mathrm{A} / \mathrm{m})$ on the horizonal axis. However, in the electrical equivalent circuit model, the magnetization inductance is represented by a piecewise linear $\lambda$-i curve. As explained in Section 4.3, it is possible to convert magnetic induction 
to flux linked ( $\lambda$ in Wb-turn) and magnetizing force to current ( $\mathrm{i}$ in $\mathrm{A})$. The scaling factors are given as:

$$
\lambda=B \times A \times N
$$

Where, $B=$ the magnetic induction in Tesla, $A=$ the core cross section in $m^{2}$ $N=$ the number of winding turns of the winding the induction is referred to.

The relation between magnetizing force and current is given as:

$$
i=H \times L
$$

where, $H=$ Magnetizing force in $A / m, i=$ current in ameperes

$L=$ the length of the flux path through the core in meters.

Figure 6.9 shows the $\lambda$-i curve for each core section of the example transformer.

Figure 6.10 shows the current waveforms of core sections at $100 \%$ voltage simulated using MATLAB. Figure 6.11 shows the current waveforms of lines at $100 \%$ voltage simulated using MATLAB.

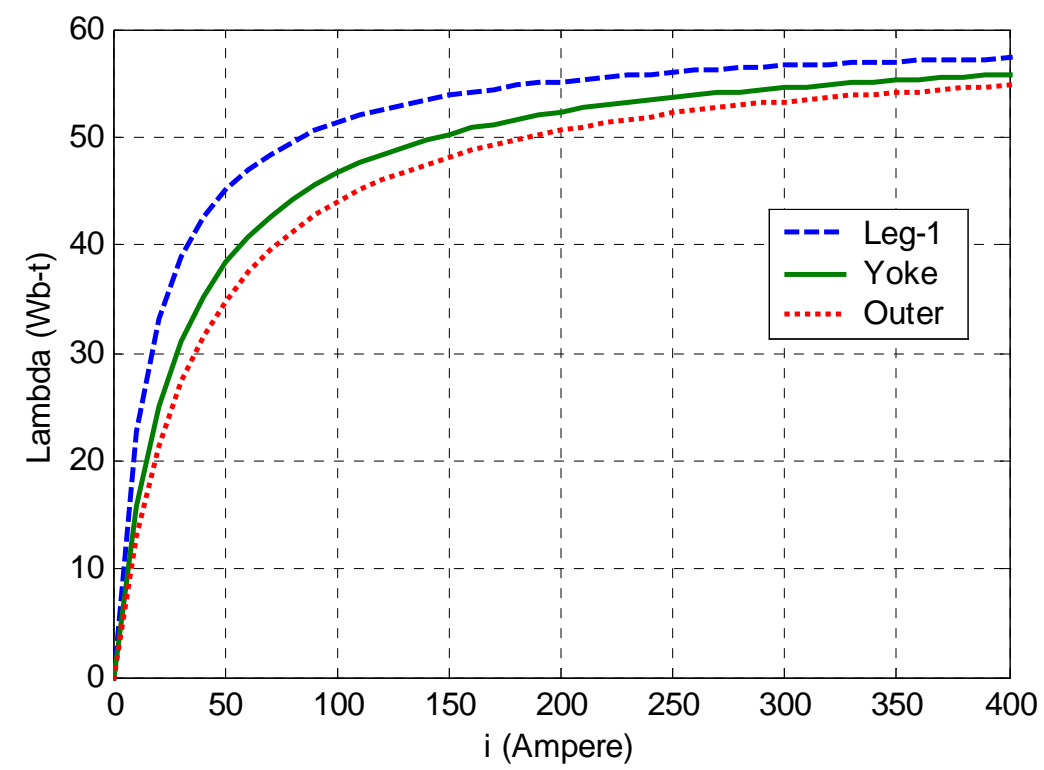

Figure 6.9 $\lambda-i$ Curve for Each Section 


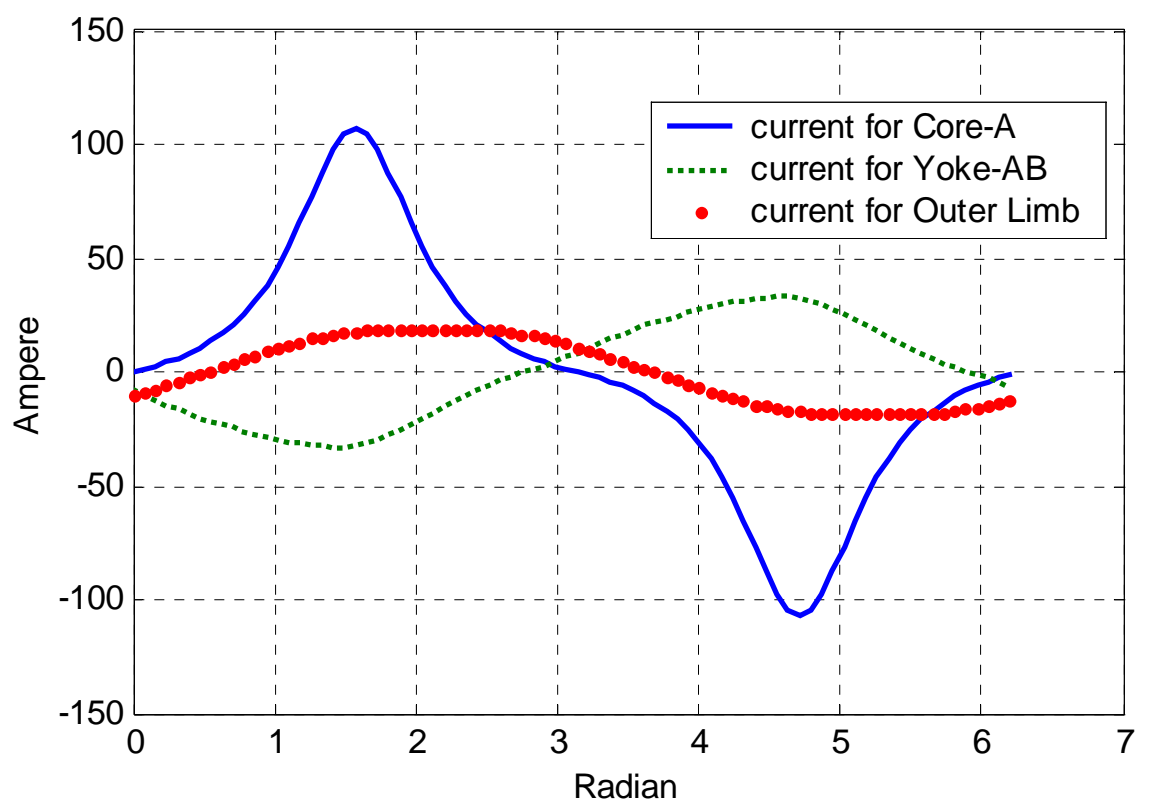

Figure 6.10 Current Waveforms for Each Section at 100\%v V

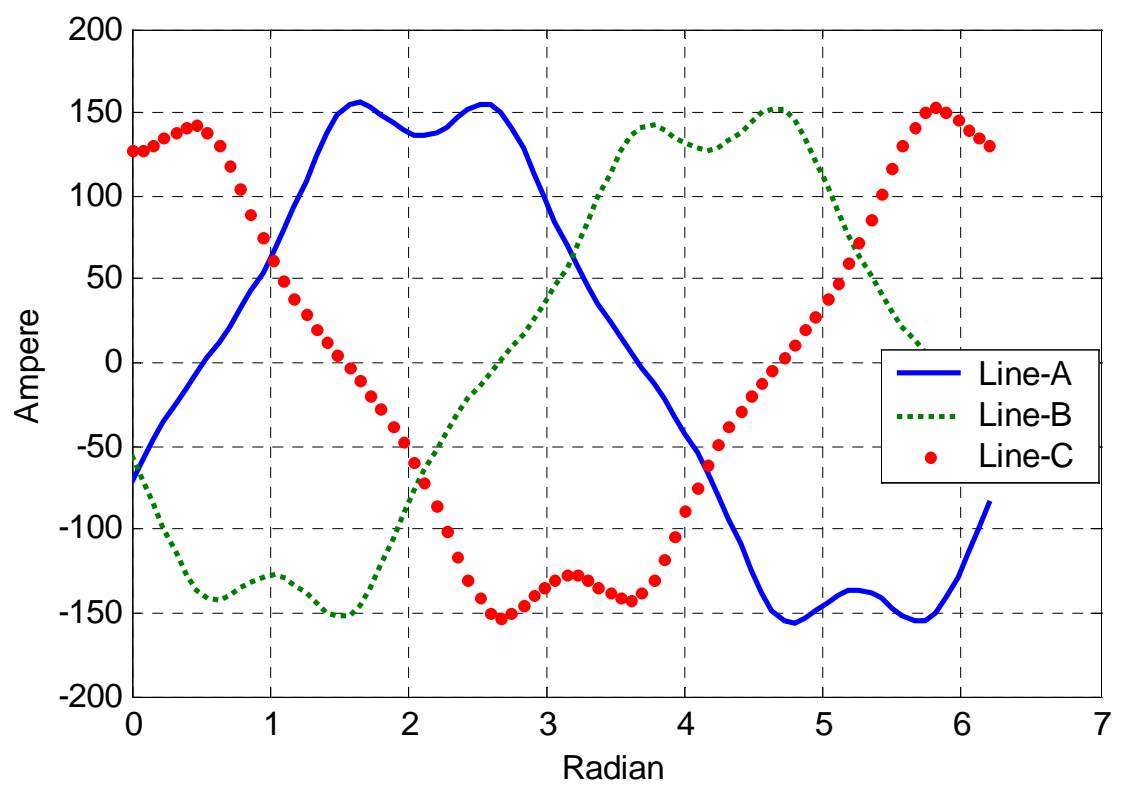

Figure 6.11 Current Waveforms for Each Line at 100\% Voltage 


\subsubsection{Core Loss Model}

From the dimensions of the legs and yokes, core volumes can be calculated. If the normalized volumes of legs and yokes are known and the normalized magnitudes of flux in core legs and yokes are known, the characteristic of the average core loss represented by the Frolich equation can be obtained. The equations for core loss curve are:

$$
P c=\frac{a \cdot B}{1-B \cdot b}
$$

From the example transformer test data, core losses at $100 \%$ voltage and $110 \%$ voltage are given as $[297600,402240](\mathrm{W})$ at $\lambda=[51.77,56.95](\mathrm{Wb}-\mathrm{t})$.

Therefore, the calculated core loss should be:

$$
\sum_{n=1}^{7} P_{n}\left(B_{n}\right) \cdot A_{n} \cdot L_{n}=\sum_{n=1}^{7} \frac{a \cdot B_{n}}{1-b \cdot B_{n}} \cdot A_{n} \cdot L_{n} \quad n \text { : core section number }
$$

Minimize $f(a, b)$

$$
=\left[P @ 100 \% V-\sum_{n=1}^{7} \frac{a \cdot B_{n}}{1-b \cdot B_{n}} \cdot A_{n} \cdot L_{n}\right]^{2}+\left[P @ 110 \% V-\sum_{n=1}^{7} \frac{a \cdot B_{n}}{1-b \cdot B_{n}} \cdot A_{n} \cdot L_{n}\right]^{2}
$$

\section{Subject to inequality constraints $0<a$ and $0<b<1$}

The normalized flux density $\mathrm{B}$ and normalized dimensions are given in the previous section, but are repeated here for convenience.

\begin{tabular}{|c|c|c|c|c|c|c|c|}
\hline & Leg-1 & Leg-2 & Leg-3 & Yoke-12 & Yoke-23 & Outer-1 & Outer-2 \\
\hline Core No & 1 & 2 & 3 & 4 & 5 & 6 & 7 \\
\hline Area & 1 & 1 & 1 & 1 & 1 & 1 & 1 \\
\hline Length & 1 & 1 & 1 & 1.725 & 1.725 & 2.21 & 2.21 \\
\hline B @ 100\%V & 1.523 & 1.523 & 1.523 & 0.951 & 0.951 & 0.608 & 0.608 \\
\hline B @ 110\%V & 1.675 & 1.675 & 1.675 & 1.031 & 1.031 & 0.673 & 0.673 \\
\hline
\end{tabular}


Thus, values of $\mathrm{a}=11567$ and $\mathrm{b}=0.4694$ are calculated for core loss $\mathrm{P}$ vs. flux density B curve using the optimization technique (Appendix B.3) from Equation (6.29). In this case, there is only a minor difference [0.0684 0.0422] $\mathrm{W}$ between the given loss and the calculated loss, verfying the correctness of this method for this case. Figure 6.12 shows the core loss curve for the five-legged core transformer.

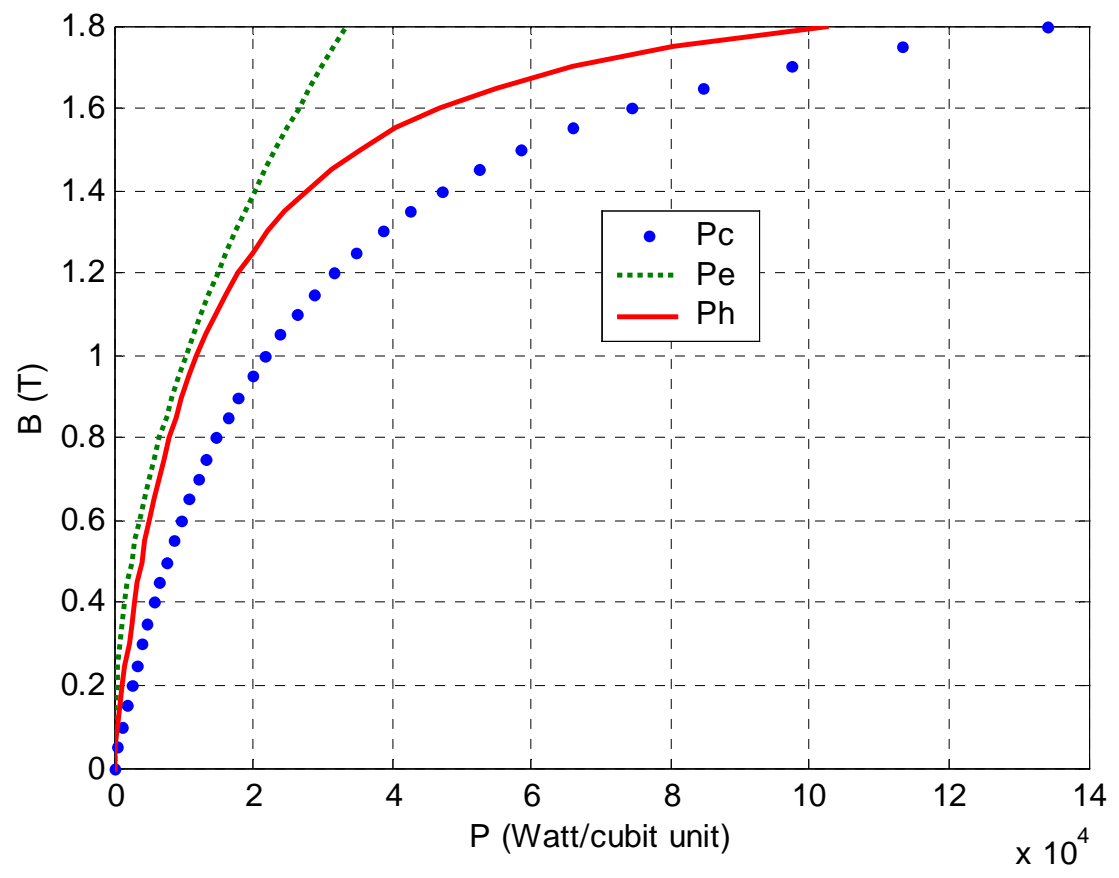

Figure 6.12 Core Loss Curve for Five-legged Core Transformer

To define the frequency-dependent effects of core loss, the core loss $\mathrm{P}_{\mathrm{C}}$ at any given frequency is generally given as below. If the ratios of hysteresis loss to total core loss $(\alpha)$ and eddy current loss to total core loss $(\beta)$ are given, the nonlinear and frequency-dependent core loss at voltage $\mathrm{V}$ and frequency $\mathrm{f}$ were defined in Section 4.5 and repeated here for convenience: 


$$
\begin{aligned}
& P_{C}=P_{H}+P_{E}=\alpha \cdot f+\beta \cdot f^{2} \\
& P_{H}=\alpha \cdot P(100 \% \mathrm{~V}) \cdot(\lambda(V) / \lambda(100 \% \mathrm{~V}))^{K} \cdot(f / 60) \\
& P_{E}=\beta \cdot P(100 \% \mathrm{~V}) \cdot(\lambda(V) / \lambda(100 \% \mathrm{~V}))^{2} \cdot(f / 60)^{2}
\end{aligned}
$$

where $P_{H}$ is hysteresis loss and $P_{E}$ is eddy current loss

If the core losses $\left(P_{1}\right.$ and $\left.P_{2}\right)$ at two frequencies $\left(f_{1}\right.$ and $\left.f_{2}\right)$ are given instead, the ratios " $\alpha$ " for hysteresis loss and " $\beta$ " for eddy current loss are defined as $\alpha=0.5245, \beta=$ 0.4755 for the example transformer:

$$
\alpha=\frac{P_{1} \cdot{f_{2}}^{2}-P_{2} \cdot f_{1}{ }^{2}}{f_{1} f_{2} \cdot\left(f_{2}-f_{1}\right)} \quad \text { and } \beta=\frac{P_{2} \cdot f_{1}{ }^{2}-P_{1} \cdot f_{2}{ }^{2}}{f_{1} f_{2} \cdot\left(f_{2}-f_{1}\right)}
$$

Using the above result, the terms for separated core loss are represented as:

$$
\begin{aligned}
R_{E} & =V^{2} /\left(P_{E} \cdot A \cdot L\right)=(V @ B=1.523)^{2} /\left[\beta \cdot\left(P_{C} @ B=1.523\right) \cdot(A \cdot L)\right] \\
& =(13800)^{2} /(0.4755 \cdot 61790) /(A \cdot L) \\
& =(6482) /(A \cdot L) \quad(\text { ohms })
\end{aligned}
$$

The equation for DC hysteresis loss from Section 4.5, is repeated here as Equation (6.31). From the core loss separation and Equation (6.31), "aa" and "bb" are obtained as $a a=6045$ and $b b=0.4964$ for the example transformer. The right displacement in the hysteresis loop is linear and assumed as in Equation (6.32). The left displacement is nonlinear and assumed as in Equation (6.33). At zero flux, both displacements must be the same. This is a coercive force $\left(\mathrm{H}_{\mathrm{C}}\right)$ and is assumed as in Equation (6.35). The coercive force at each loop should be determined to meet the power loss $\mathrm{P}_{\mathrm{H}}$ at the $\mathrm{B}_{\max }$ for the each loop. In case of Figure 6.13, "k" for Equation (6.35) is about 0.5 and $\mathrm{H}_{\text {ctop }}$ is about $2.7 \mathrm{~A}$. 
The displacements at each Bmax are shown in Figure 6.14. The entire DC hysteresis loop is shown in Figure 6.15.

$P_{H}\left(B_{M A X}\right)=P_{C}\left(B_{M A X}\right)-P_{E}\left(B_{M A X}\right)=\frac{a a \cdot B_{M A X}}{1-b b \cdot B_{M A X}}$

Right displacement $R H D=(1-f) \cdot H_{C}$

Left displacement $\left.L H D=-H_{C} \cdot(a+1 / a) /[(1-f) / a+a /(1-f)]\right)$

Power Loss at each loop $(J)=A \cdot L \cdot N \cdot \int_{0}^{B_{\max }} 2 \cdot(R H D-L H D) \cdot d B$

Power Loss at each loop $(W)=60 \cdot A \cdot L \cdot N \cdot \int_{0}^{B_{\max }} 2 \cdot(R H D-L H D) \cdot d B$

Coercive force $H c=\left(B_{\text {max }} / B_{\text {top }}\right)^{K} \times H_{\text {ctop }}$

where

$B_{\text {max }}=$ Maximum Flux density at each minor loop

$B_{\text {top }}=$ Maximum Flux density for major loop (Given)

$a=\left(B_{\text {top }}-B_{\text {max }}\right) / B_{\text {top }}$ and $f=B / B_{\max }$

$H_{\text {ctop }}=$ Maximum Coercive force for major loop

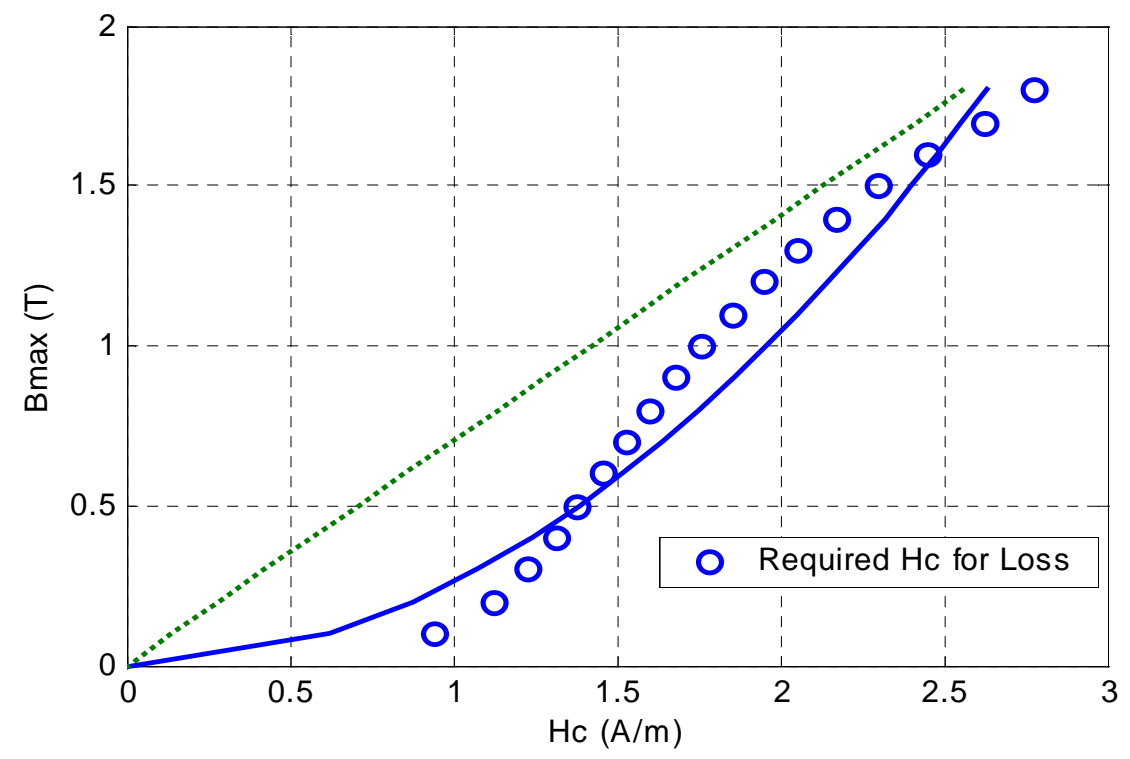

Figure 6.13 $\mathrm{H}_{\mathrm{C}}$ and $\mathrm{B}_{\max }$ (dotted line=linear, bold line=square root) 


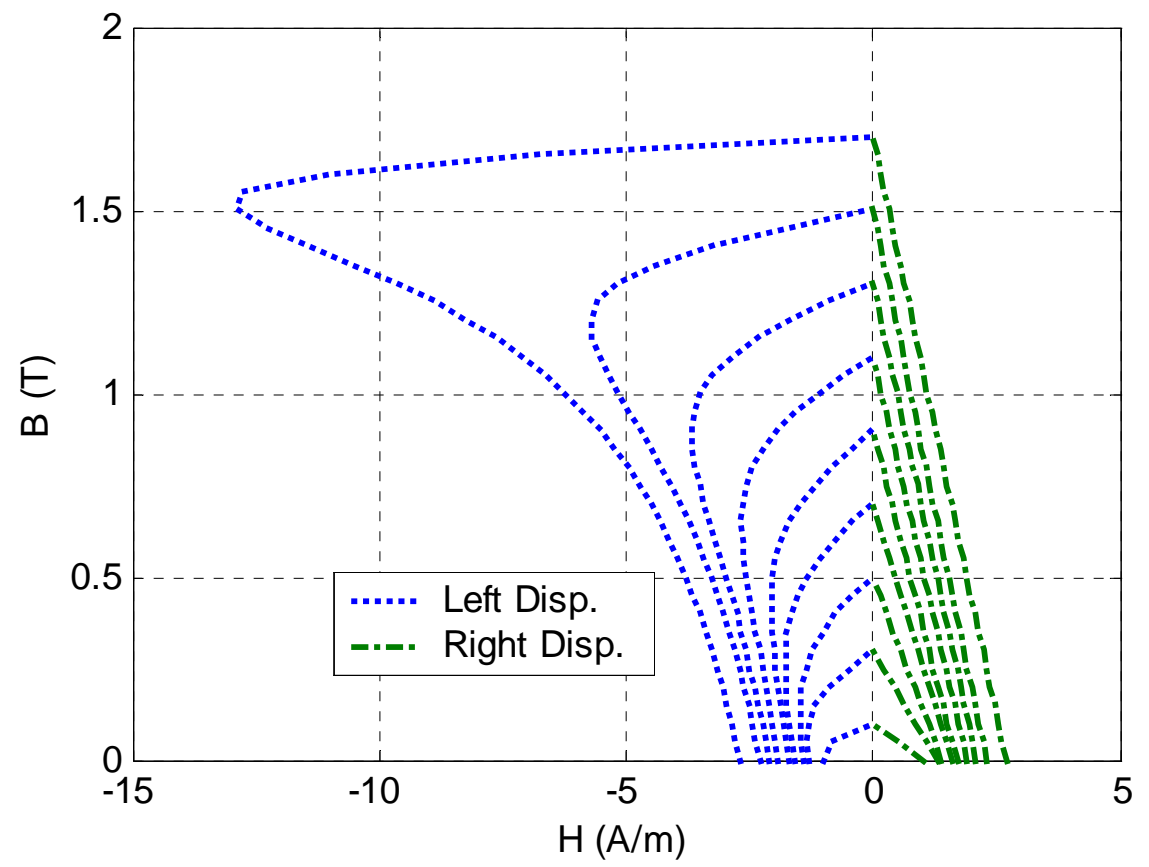

Figure 6.14 Left and Right Displacements of Resistive Hysteresis Current

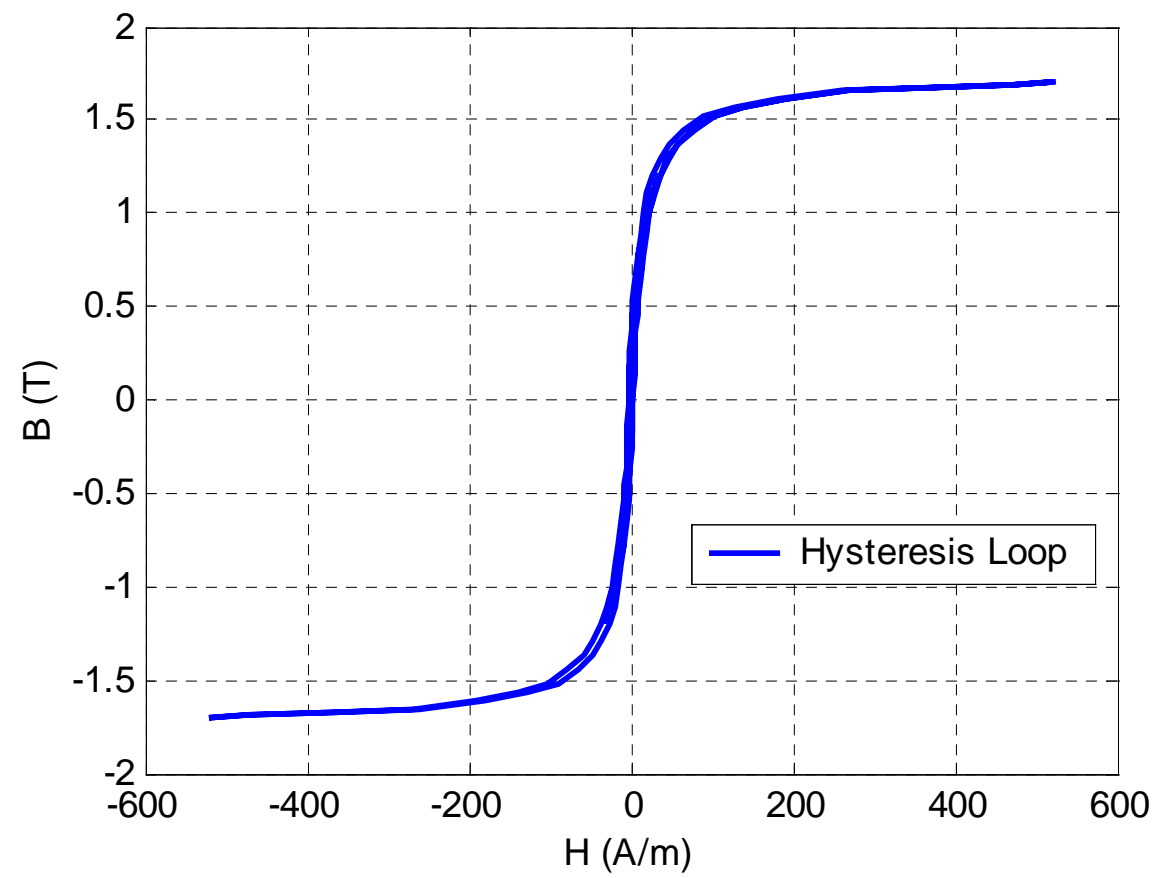

Figure 6.15 DC Hysteresis Loop Generated by the Model 


\subsubsection{ATP Implementation of Overall Transformer Model}

The overall transformer model for ATP implementation is shown in Figure 6.17. The core model, frequency-dependent coil resistance and winding capacitances developed in Chapter 4 are included in ATP format.

\section{Ideal Transformer Coupling}

The coupling between windings is provided by using "Type-18" ideal transformer elements or saturable transformer elements. There are nine of these elements in the threewinding three-phase transformer model in Figure 6.17. Since the parameters of the core equivalent are referred to the tertiary, the tertiary coupling transformers have a turn ratio of unity. The ideal transformers coupling the primary and medium-voltage coils to the core have a turns ratio equal to their actual turns ratio with respect to the tertiary.

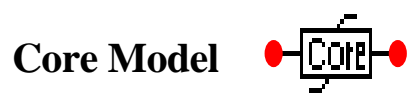

The core model is of most interest in this work and includes the saturable magnetizing inductances, hysteresis losses, and eddy current losses of core legs and yokes. The core model implemented in ATP was shown in Figure 4.32.

There are three nonlinear inductances available in ATP. The "Type-93" was chosen for this work as it is a true nonliear inductance [30]. Operation is always on the proper $\lambda-i$ segment of the charateristic and hence may allow much better results [17]. The Type-96 hysteretic inductance and the Type-98 pseudo-nonlinear inductances are not as robust, due to different implementation methods. 
The hysteresis losses and eddy current losses of core legs and yokes are modeled using a Type-60 current source controlled by TACS. The block diagram related for TACS code was shown in Figure 4.33.

\section{Coil Resistance Model, R(f)}

A Foster equivalent, as developed in Section 4.1, is used to represent frequencydependency resistance. Figure 6.16 shows the actual frequency-dependency resistance model implemented inside R(f). In this model, three resisters and three inductors are used for the Foster equivalent circuit with two cells. The third inductance, $\mathrm{L}_{3}$ is a negative inductance for removing effective inductance given by $\mathrm{L}_{1}$ and $\mathrm{L}_{2}$. The negative inductance may in some cases give a numerical stability problem in ATP simulations. Therefore, incorporating circuit components based on Foster equivalents into the leakage inductance matrix should be further studied to improve numerical stability.

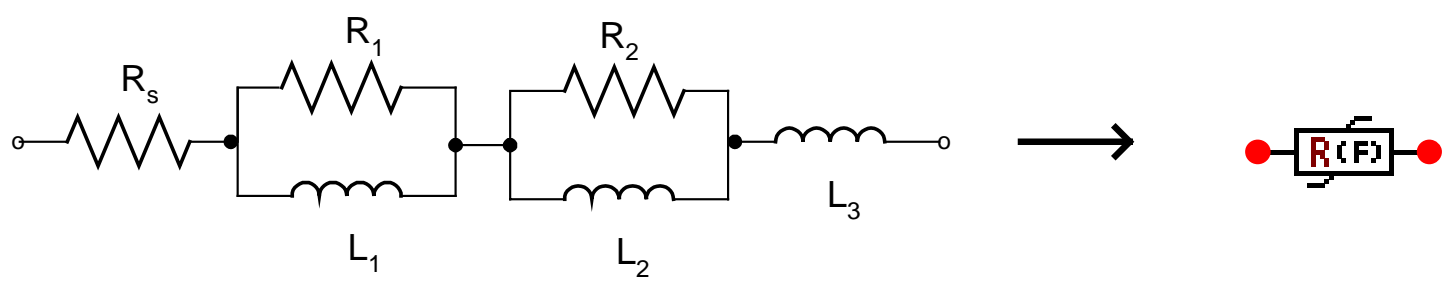

Figure 6.16 Frequency-Dependency Resistance Model R(f) Implementation in ATP

\section{Dummy Resistance and Inductance}

A large resistance "Rd" was added to avoid floating subnetwork problems, as shown in Figure 6.17. Two nonlinear inductances connected in series at a node may cause ATP to report an error. Hence, small linear inductances "Xd" are added to separate two nonlinear inductances. 


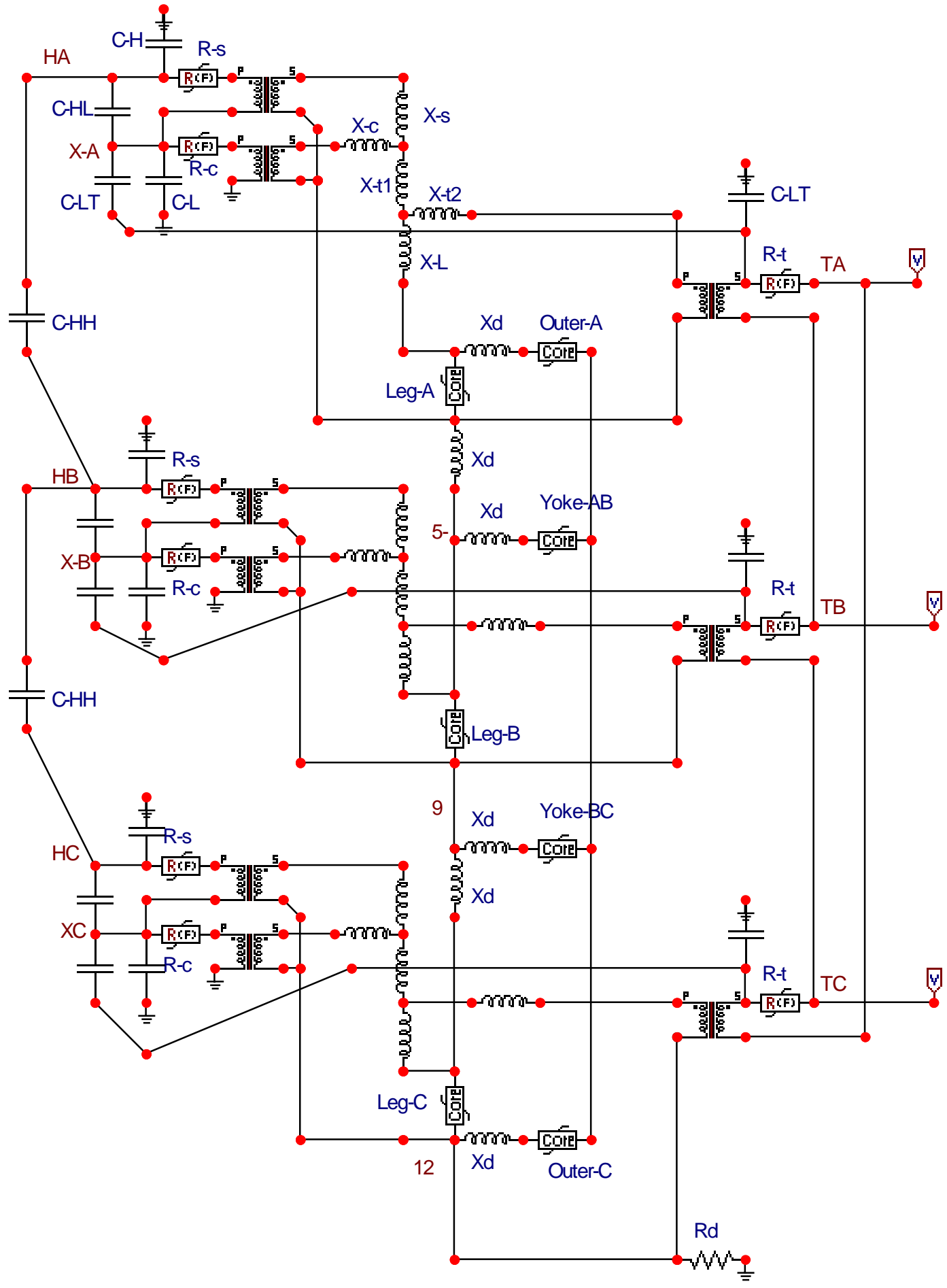

Figure 6.17 Equivalent Circuit for Five-Legged Core Transformer, implemented in ATP 
Figure 6.18 shows the DC hysteresis loop modeled using a Type-60 current source controlled by TACS. Figure 6.19 shows the current of the eddy current loss and the resistive hysteresis current. Figure 6.20 shows the magnetizing current modeled with a Type-93 nonlinear inductance. Figures 6.21 and 6.22 show the line-current and windingcurrent waveforms.

After all models were implemented and run with ATP, the results of open-circuit and short-circuit simulations shown in Table 6.3 are close to the test report.

Table 6.3 Comparisons with Test Report

\begin{tabular}{|c|c|}
\hline Test Report & Simulated Results \\
\hline \multicolumn{2}{|c|}{ Excitation Current @ 13.8kV Line } \\
\hline 94.12 $\mathrm{A}_{\mathrm{RMS}} @ 100 \%$ Voltage & $100.53 \mathrm{~A}_{\mathrm{RMS}} @ 100 \%$ Voltage \\
\hline $211.76 \mathrm{~A}_{\mathrm{RMS}} @ 110 \%$ Voltage & $208.1 \mathrm{~A}_{\mathrm{RMS}} @ 110 \%$ Voltage \\
\hline \multicolumn{2}{|c|}{ No Load Loss } \\
\hline $297.6 \mathrm{~kW} @ 100 \%$ Voltage & $306.7 \mathrm{~kW} @ 100 \%$ Voltage \\
\hline $402.24 \mathrm{~kW} @ 110 \%$ Voltage & $383.5 \mathrm{~kW} @ 110 \%$ Voltage \\
\hline \multicolumn{2}{|c|}{ Short-Circuit Current } \\
\hline $495.3 \mathrm{~A}_{\mathrm{RMS}} @ \mathrm{P}-\mathrm{S}$ & $495.3 \mathrm{~A}_{\mathrm{RMS}} @ \mathrm{P}-\mathrm{S}$ \\
\hline $128.9 \mathrm{~A}_{\mathrm{RMS}} @ \mathrm{P}-\mathrm{T}$ & $128.9 \mathrm{~A}_{\mathrm{RMS}} @ \mathrm{P}-\mathrm{T}$ \\
\hline $367.7 \mathrm{~A}_{\mathrm{RMS}} @ \mathrm{~S}-\mathrm{T}$ & $367.7 \mathrm{~A}_{\mathrm{RMS}} @ \mathrm{~S}-\mathrm{T}$ \\
\hline
\end{tabular}




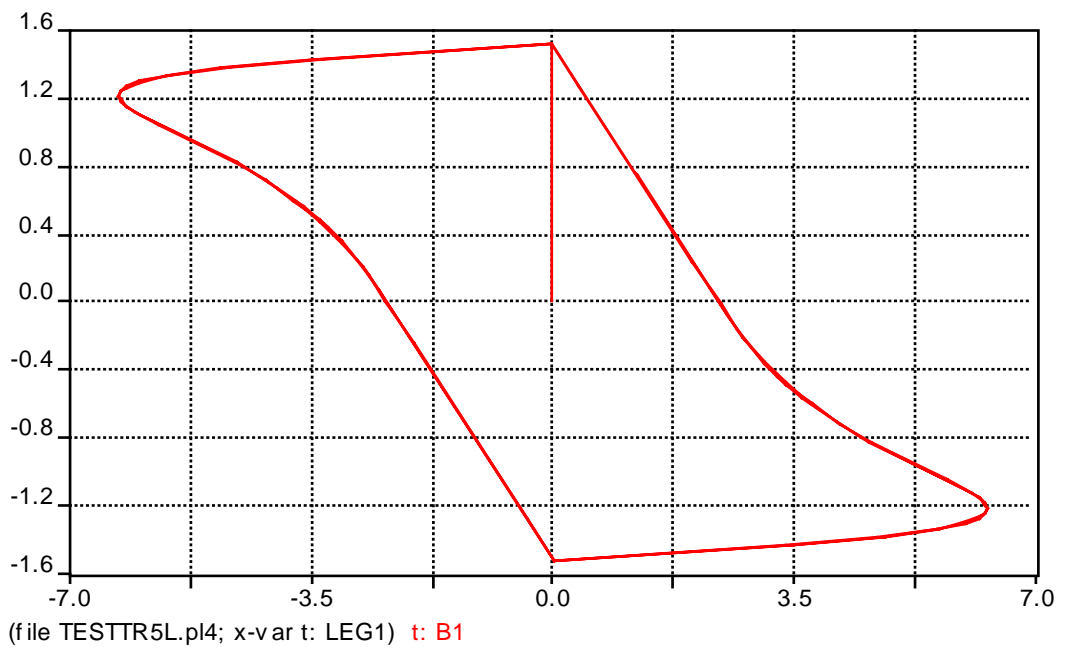

Figure 6.18 DC Hysteresis Loop Generated by ATP

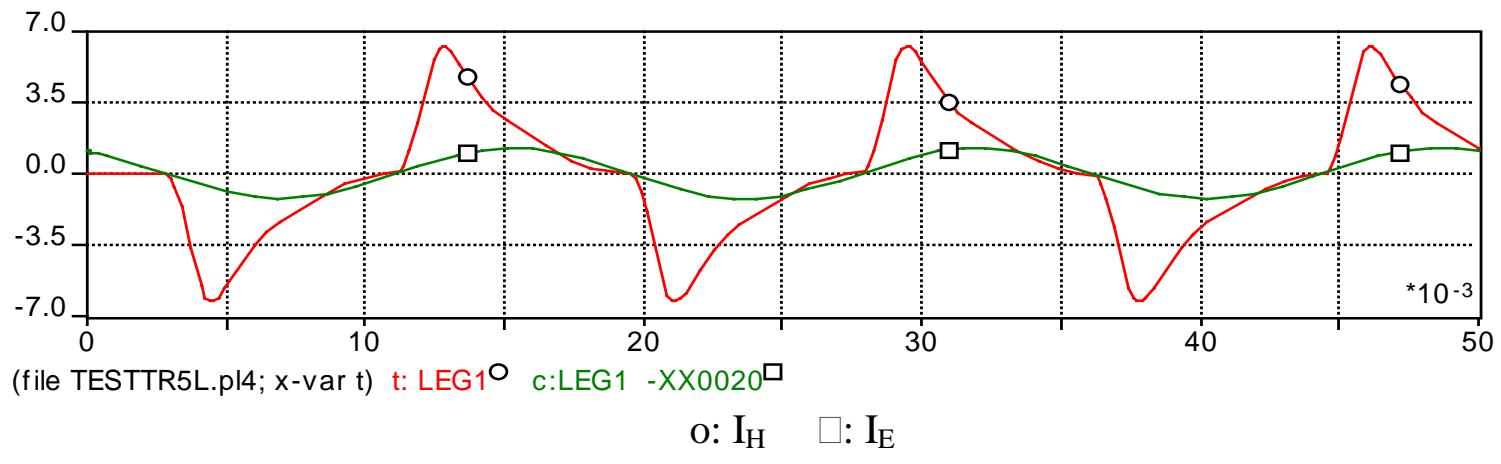

Figure 6.19 Eddy Current $\left(\mathrm{I}_{\mathrm{E}}\right)$ and Hysteresis Current $\left(\mathrm{I}_{\mathrm{H}}\right)$ Waveforms at $100 \% \mathrm{~V}$

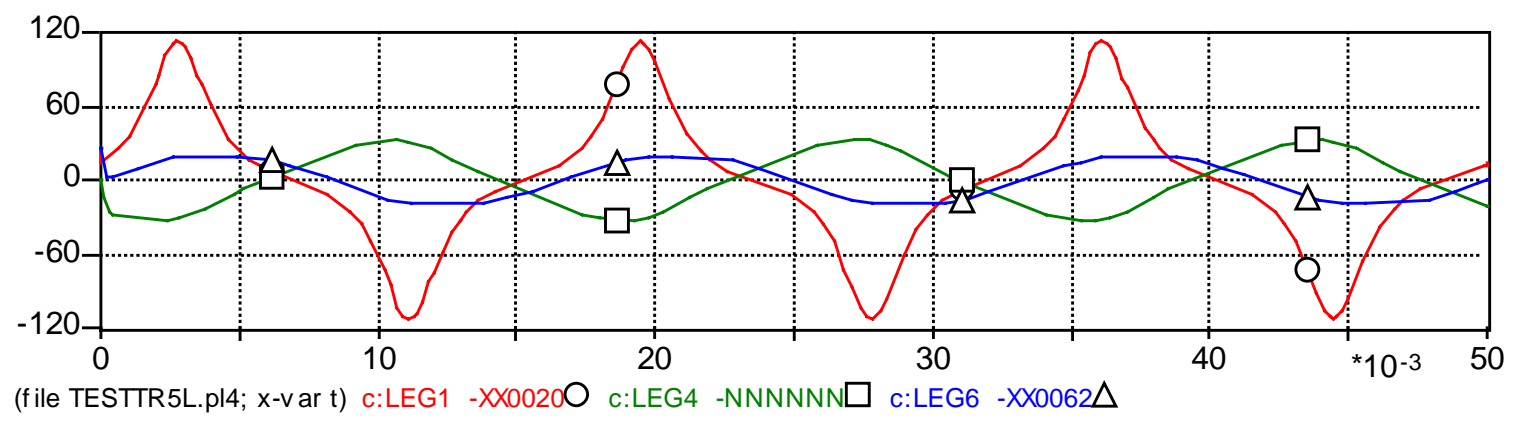

o: Leg-1 $\Delta$ : Yoke-AB $\square$ : Outer Limb at $100 \% \mathrm{~V}$

Figure 6.20 Mangnetizing Current Waveforms of Leg- 1, Yoke-AB and Outer Limb 


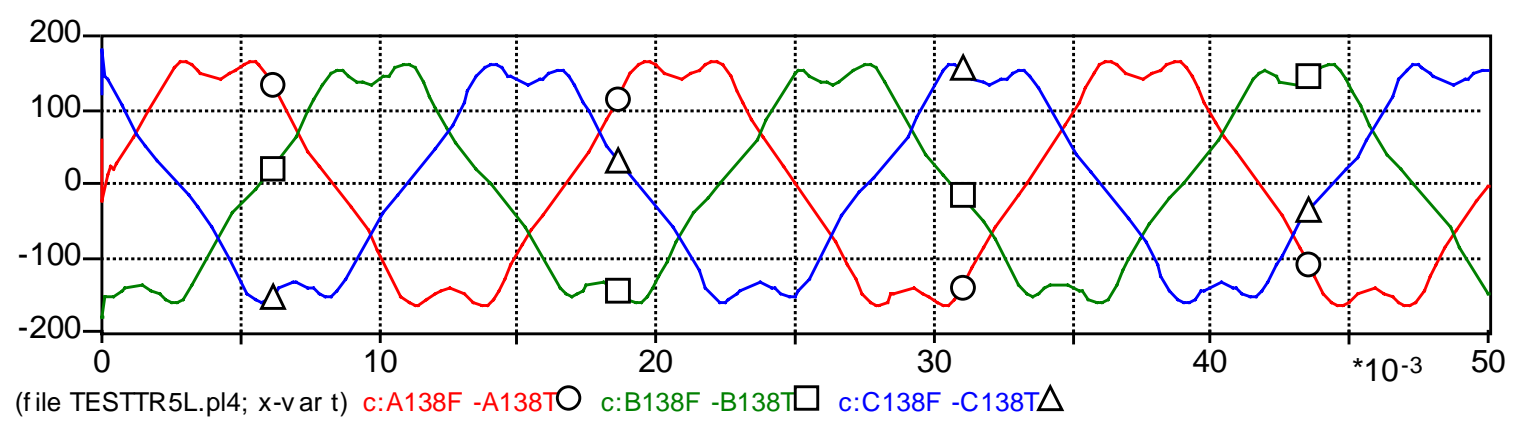

o: Line-A $\square$ : Line-B $\quad \Delta$ : Line-C

Figure 6.21 Line Current Waveforms for Tertiary at 100\% Voltage

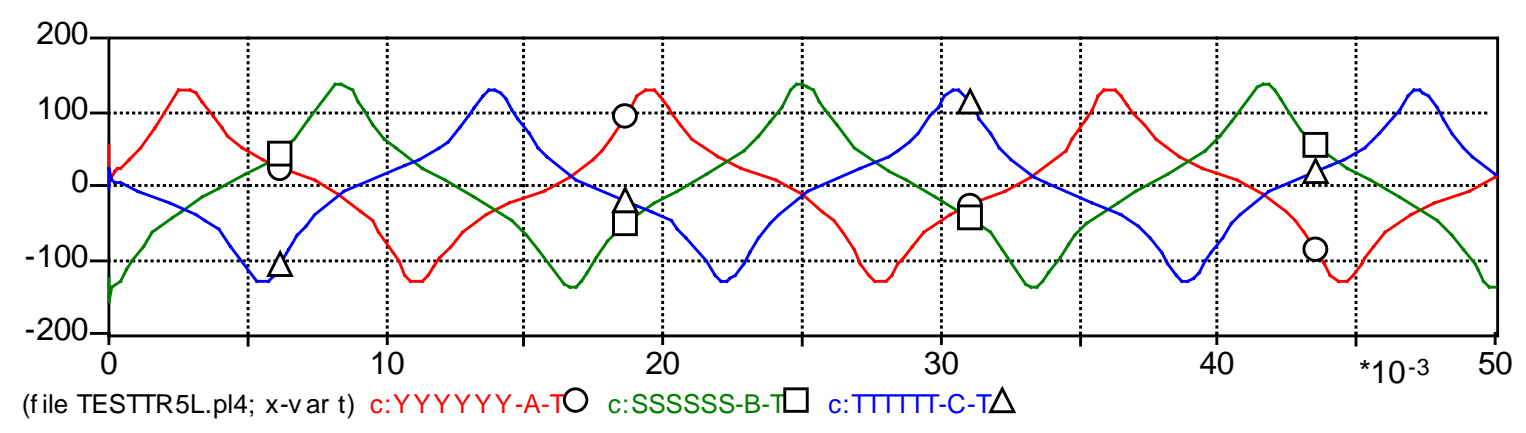

o: Phase-A $\square$ : Phase-B $\Delta$ : Phase-C

Figure 6.22 Phase Current Waveforms for Tertiary at 100\% Voltage 


\subsection{Three-Legged Core Transformer}

\subsubsection{Leakage Inductance}

Leakage paths and inductances for three-phase three-legged core-form transformers are the same as those of three-phase five-legged core-form transformers except for the zero sequence flux. The zero sequence impedance comes from the zero sequence test. It is difficult to calculate the zero sequence parameters without finite element analysis because the zero sequence flux path is through the transformer tank. Hence, values obtained from measurements should be used if available.

An autotransformer usually has a closed-delta tertiary and this tertiary gives a path for zero sequence current. Therefore, the model for zero sequence flux path through the tank is not required for an autotransformer with a closed delta tertiary.

\subsubsection{Core Saturation Model}

For the three-legged transformer, core area is assumed to be the same as those of the legs in a five-legged transformer and the core length is assumed as in Figure 6.23. The magnetic equivalent circuit is given in Figure 6.24. All reluctances here are saturable. Only one set of MMFs (windings) is included, typical of no-load excitation. Fluxes are defined such that $\Phi_{1}, \Phi_{2}$, and $\Phi_{3}$ are the fluxes in the 3 legs. The procedure for core saturation model derivation is the same as the procedure in Section 6.1.3. 


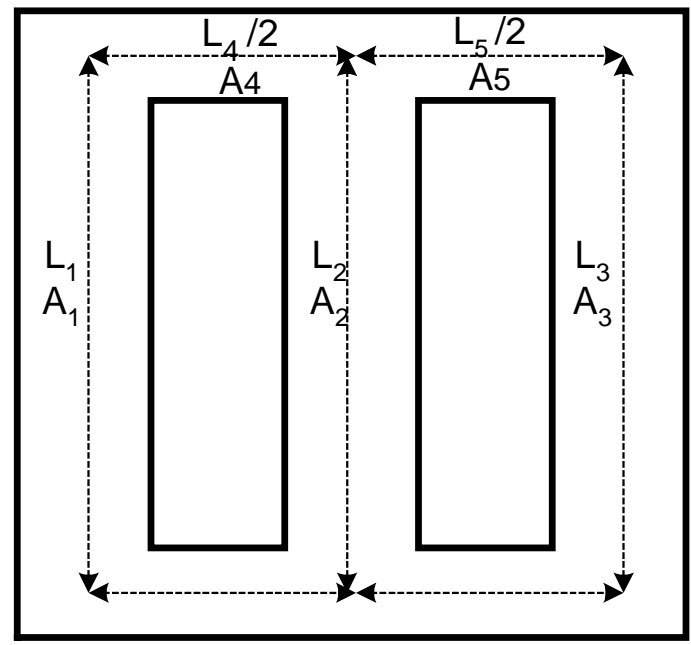

\begin{tabular}{|c|c|}
\hline Area ratio & Length ratio \\
\hline$A_{1}=A_{2}=A_{3}=1$ & $L_{1}=L_{2}=L_{3}=1$ \\
\hline Yoke $\mathrm{A}_{4}=\mathrm{A}_{5}=1$ & ${\text { Yoke } \mathrm{L}_{4}=\mathrm{L}_{5}=1.725}$ \\
\hline
\end{tabular}

Notation: A: Area, L: Length, 1: Leg-A, 2:Leg-B, 3: Leg-C, 4:Yoke-AB, 5: Yoke-BC

Figure 6.23 Dimension of Three-Legged Core Type Transformer

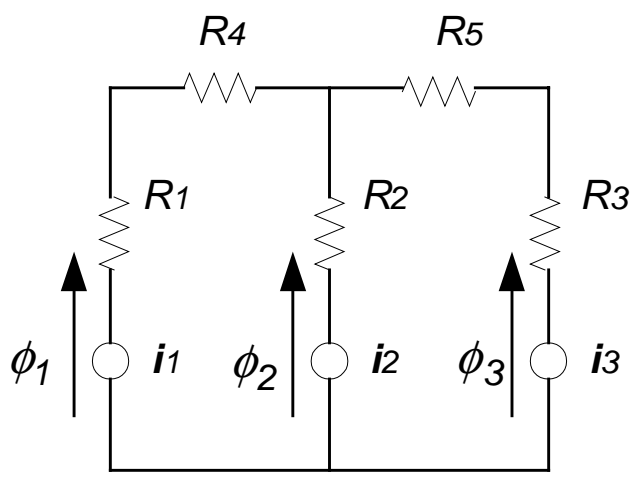

Figure 6.24 Magnetic Equivalent Circuit for Three-Legged Transformer 


$$
\begin{aligned}
& i_{1}=\phi_{1}\left(R_{1}+R_{4}\right) \\
& i_{2}=\phi_{2} R_{2} \\
& i_{3}=\phi_{3}\left(R_{3}+R_{5}\right)
\end{aligned}
$$

Where,

$$
\begin{array}{ll}
R_{1}=L_{1} /\left(\mu_{1} A_{1}\right) & \mu_{1}=\phi_{1} /\left(A_{1} H_{1}\right)=f\left(\phi_{1} / A_{1}\right) \\
R_{2}=L_{2} /\left(\mu_{2} A_{2}\right) & \mu_{2}=\phi_{2} /\left(A_{2} H_{2}\right)=f\left(\phi_{2} / A_{2}\right) \\
R_{3}=L_{3} /\left(\mu_{3} A_{3}\right) & \mu_{3}=\phi_{3} /\left(A_{3} H_{3}\right)=f\left(\phi_{3} / A_{3}\right) \\
R_{4}=L_{4} /\left(\mu_{4} A_{4}\right) & \mu_{4}=\left(\phi_{1}\right) /\left(A_{4} H_{4}\right)=f\left(\phi_{1} / A_{4}\right) \\
R_{5}=L_{5} /\left(\mu_{5} A_{5}\right) & \mu_{5}=\left(\phi_{3}\right) /\left(A_{5} H_{5}\right)=f\left(\phi_{3} / A_{5}\right)
\end{array}
$$

\section{Known values:}

$\phi_{1}=v_{1} /(\omega N), \phi_{2}=v_{2} /(\omega N), \phi_{3}=v_{3} /(\omega N), V=$ peak-voltage for each phase, $\omega=2 \pi f$, $N=$ number of turn (In this work, 34 was assumed. This means the units of $B, H$ are per unit.)

Magnetizing Current: $I_{r m s, A V G}=\left(I_{1, r r m s}+I_{2, r r m s}+I_{3, r r m s}\right) / 3$

Note: Real component of $I_{E X}$ has been removed, as explained in Section 4.2

Core dimensions or normalized ratios

Unknown values: $a, b$ for $\mu=\frac{B}{H}=\frac{(1-b \cdot B)}{a}$

If the core dimension ratios and average RMS magnetizing currents at $100 \%$ and $110 \%$ voltages are given, optimization techiniques can be used to estimate the a and $b$ coefficients for the B-H Frolich equation from Equations (6.36) through (6.38).

The optimization performed is as follows: Details for waveform $i_{1}$ are given. Waveforms for $i_{2}$ and $i_{3}$ are obtained in a similar fashion based on Equations (6.37) and (6.38).

$$
\begin{gathered}
\text { Minimize } f(a, b)=\left[\text { Measured } I_{A V G, R M S} @ 100 \% V-\text { Calculated } I_{A V G, R M S} @ 100 \% V\right]^{2} \\
+\left[\text { Measured } I_{A V G, R M S} @ 110 \% V-\text { Calculated } I_{A V G, R M S} @ 110 \% V\right]^{2} \\
\text { Subject to inequality constraints } 0<\text { a and } 0<b<1
\end{gathered}
$$




$$
\begin{aligned}
& \text { Where, } \phi_{1}(k)=v_{1} /(\omega N) \times \sin (\pi \cdot k / 40) \\
& B_{1}(k)=\phi_{1}(k) / A_{1} \quad \text { and } B_{4}(k)=\phi_{1}(k) / A_{4} \\
& \mu_{1}(k)=\frac{1-b \cdot B_{1}(k)}{a} \text { and } \mu_{4}(k)=\frac{1-b \cdot B_{4}(k)}{a} \\
& R_{l}(k)=L_{1} /\left(\mu_{1}(k) \cdot A_{1}\right) \quad \text { and } R_{4}(k)=L_{4} /\left(\mu_{4}(k) \cdot A_{4}\right) \\
& i_{1}(k)=\phi_{1}(k) \cdot R_{1}(k)+R_{4}(k) \cdot \phi_{1}(k) \\
& I_{1, R M S}=\sqrt{\frac{\sum_{k=1}^{40}\left(i_{1}(k)\right)^{2}}{40}} \\
& I_{R M S, A V G}=\left(I_{1, R M S}+I_{2, R M S}+I_{3, R M S}\right) / 3
\end{aligned}
$$

From the optimization technique (Appendix B.1) using Fmincon, the results are $\mathrm{a}=$ $5.9265, \mathrm{~b}=0.5879$. Figure 6.25 shows the resulting B-H curve. The calculated RMS currents for the three phases are $\left[\begin{array}{lll}129.0866 & 92.9868 & 92.9868\end{array}\right] \mathrm{A}$ at $100 \%$ voltage. The calculated average RMS current is $105.02 \mathrm{~A}$ and the difference between test report is only $3.1336 \times 10^{-5} \mathrm{~A}$. The calculated RMS currents for phases are [272.7272 199.8044 199.8043 ]A at $110 \%$ voltage. The calculated average RMS current is 224.11 and the difference between test report is only $1.8260 \times 10^{-5} \mathrm{~A}$.

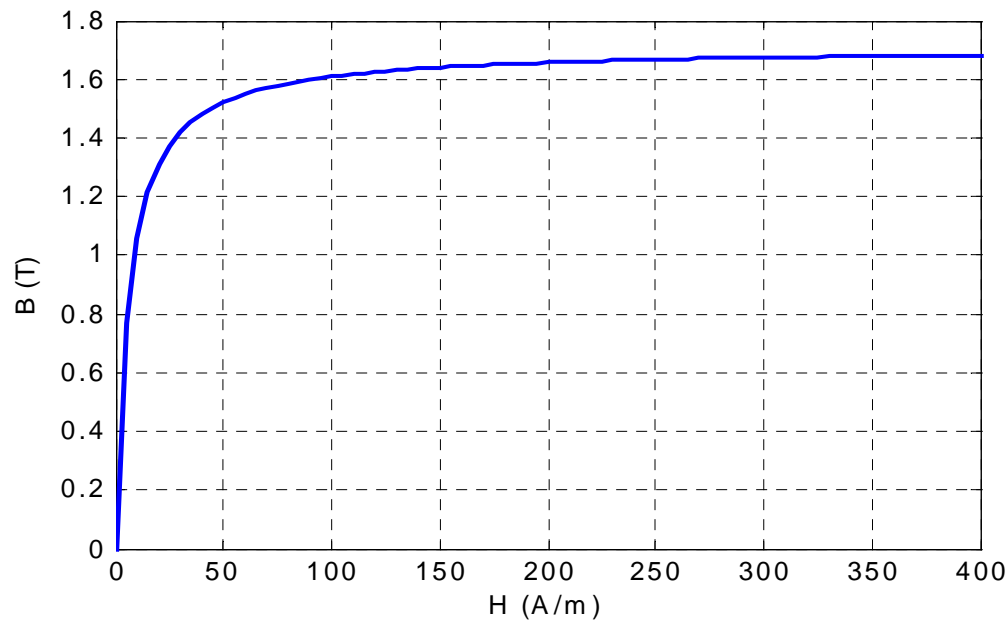

Figure 6.25 B-H Curve for Each Section 
Figure 6.26 shows the $\lambda$-i curve for each core section of the example transformer. Figure 6.27 shows the current waveforms of core sections at $100 \%$ voltage simulated using MATLAB. Figure 6.28 shows the current waveforms of lines at $100 \%$ voltage simulated using MATLAB.

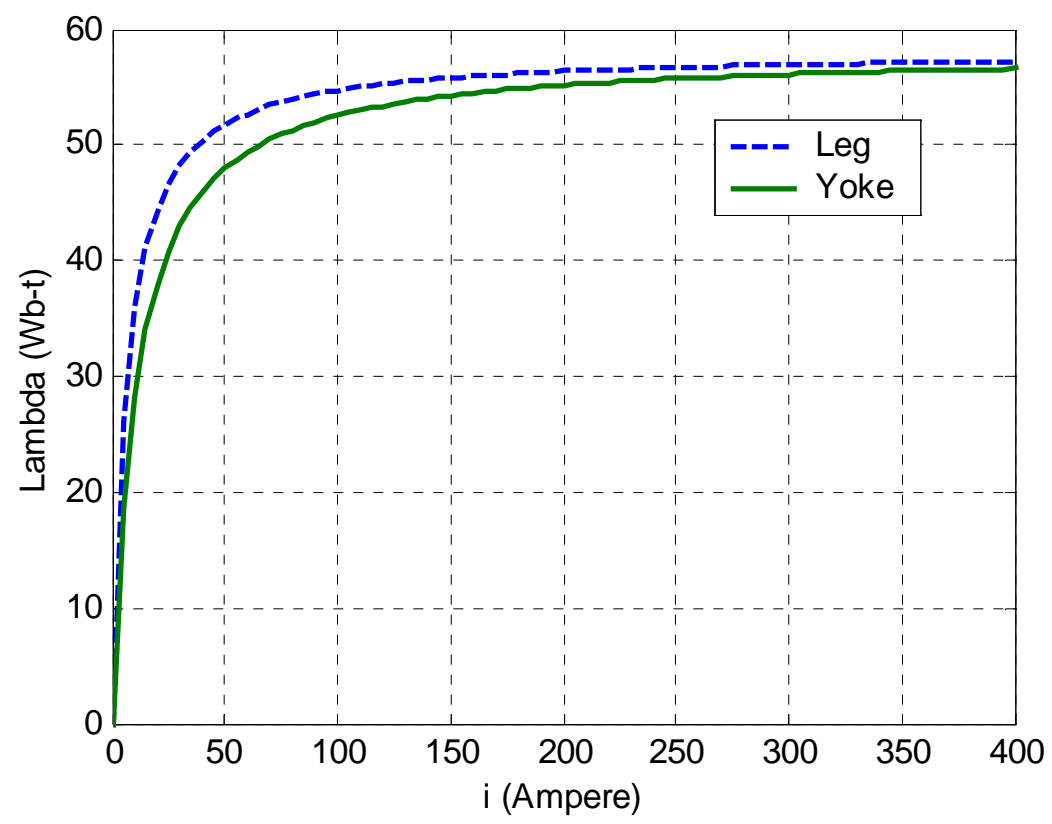

Figure 6.26 $\lambda-i$ Curves for Each Section

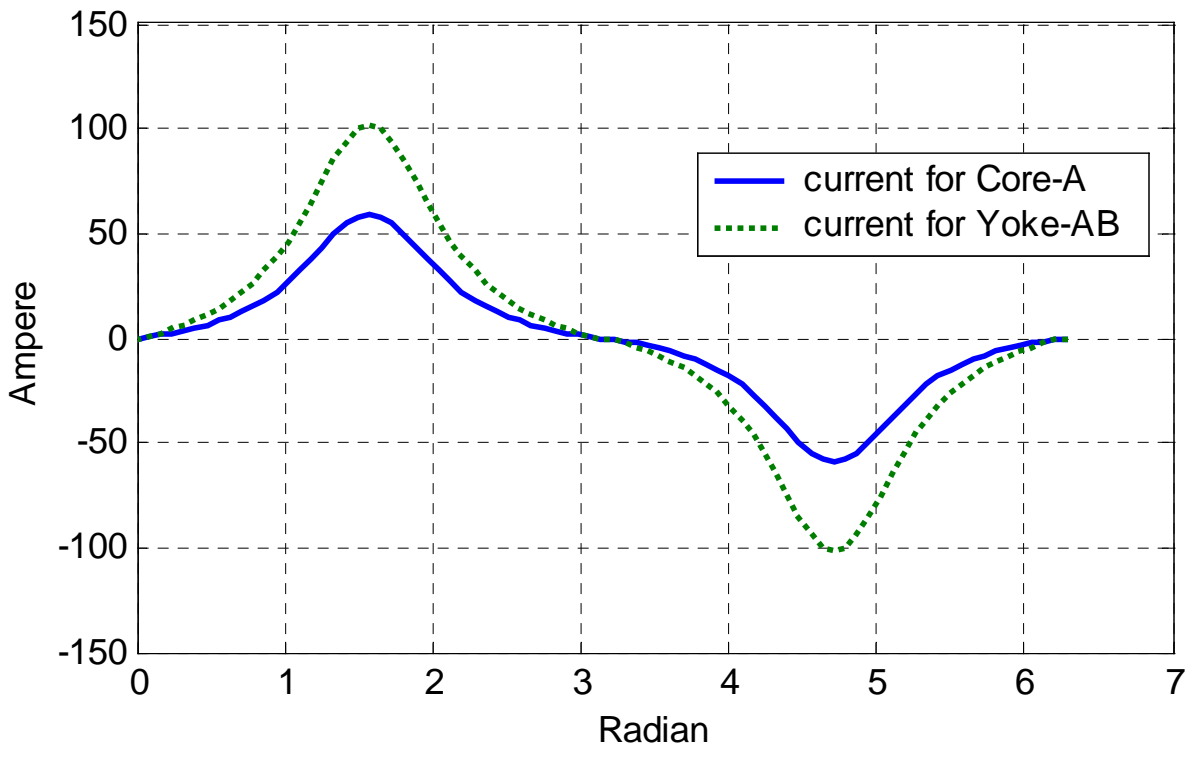

Figure 6.27 Current Waveforms for Each Section at 100\% Voltage 


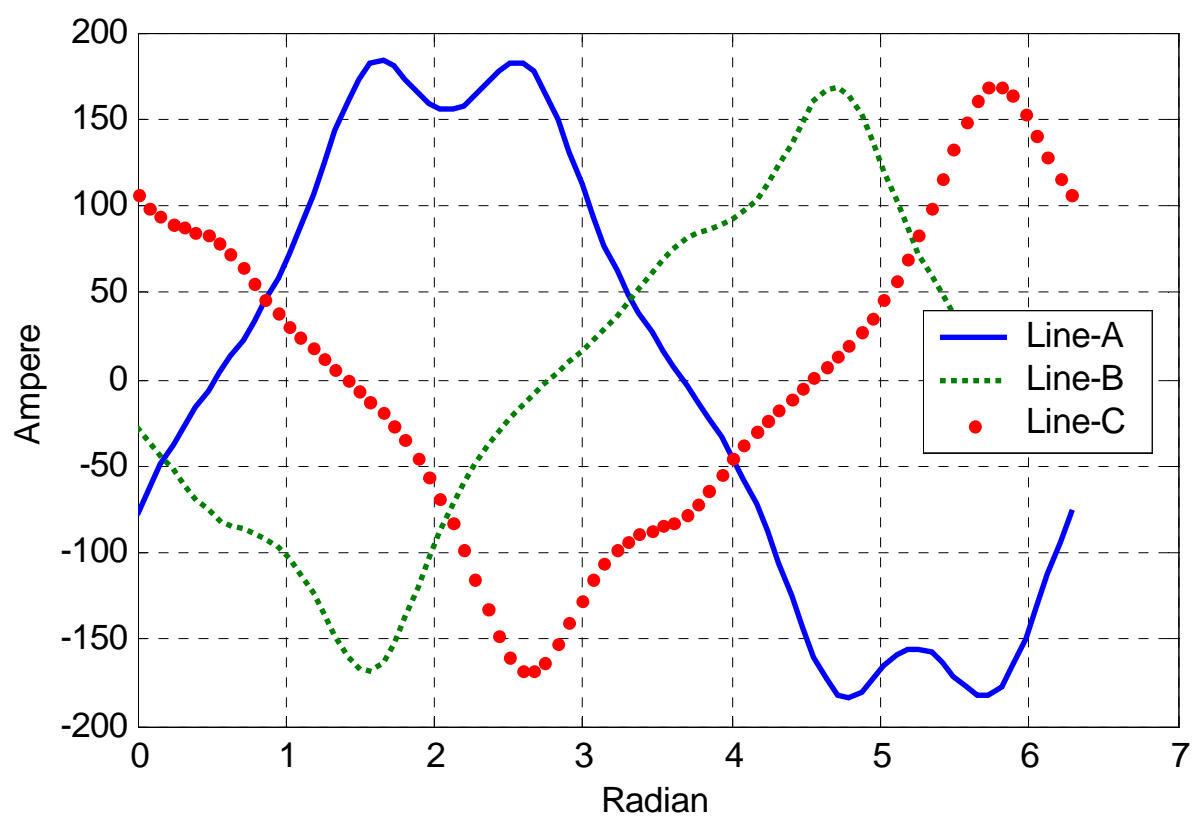

Figure 6.28 Line Current Waveforms for Tertiary at 100\% Voltage

\subsubsection{Core Loss Model}

From test data, core losses at $100 \%$ voltage and $110 \%$ voltage are known to be [297600 402240] (W) at $\lambda=[51.77,56.95](W b-t)$.

Therefore, the calculated average core loss should be:

$$
\sum_{n=1}^{5} P_{n}\left(B_{n}\right) \cdot A_{n} \cdot L_{n}=\sum_{n=1}^{5} \frac{a \cdot B_{n}}{1-b \cdot B_{n}} \cdot A_{n} \cdot L_{n}
$$

The optimization (Appendix B.10) performed is as follows:

Minimize $f(a, b)$

$$
=\left[P @ 100 \% \mathrm{~V}-\sum_{n=1}^{5} \frac{a \cdot B_{n}}{1-b \cdot B_{n}} \cdot A_{n} \cdot L_{n}\right]^{2}+\left[P @ 110 \% V-\sum_{n=1}^{5} \frac{a \cdot B_{n}}{1-b \cdot B_{n}} \cdot A_{n} \cdot L_{n}\right]^{2}
$$

Subject to inequality constraints $0<a<50000$ and $0<b<1$ 
The flux density B and normalized dimensions are also given from the previous section.

\begin{tabular}{|c|c|c|c|c|c|}
\hline & Leg-1 & Leg-2 & Leg-3 & Yoke-12 & Yoke-23 \\
\hline Core No & 1 & 2 & 3 & 4 & 5 \\
\hline Area & 1 & 1 & 1 & 1 & 1 \\
\hline Length & 1 & 1 & 1 & 1.725 & 1.725 \\
\hline Bmax @ 100\%V & 1.5226 & 1.5226 & 1.5226 & 1.5226 & 1.5226 \\
\hline Bmax @ 110\%V & 1.6749 & 1.6749 & 1.6749 & 1.6749 & 1.6749 \\
\hline
\end{tabular}

Thus, $\mathrm{a}=10592$ and $\mathrm{b}=0.4272$ for the core loss curve equation are calculated using optimization technique Fimincon from Equation (6.41). In this case, there is no difference between the given $\mathrm{P}_{\mathrm{C}}$ and the calculated $\mathrm{P}_{\mathrm{C}}$. Figure 6.29 shows the core loss curve for the three-legged core transformer.

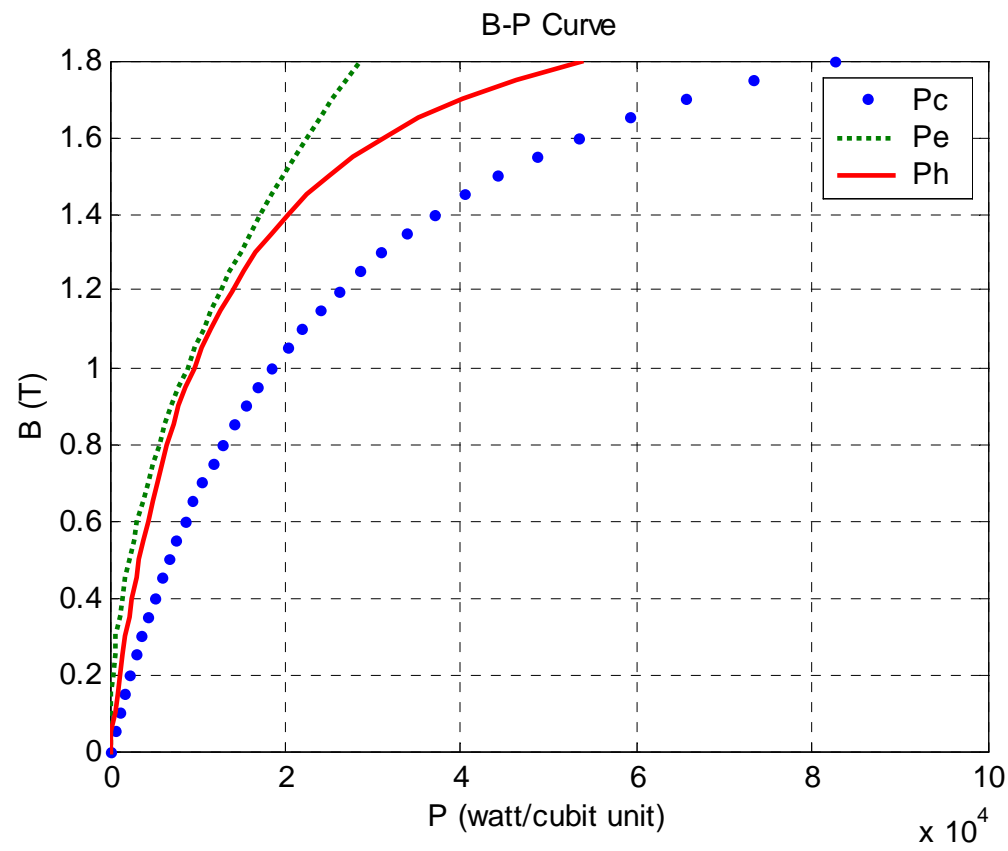

Figure 6.29 Core Loss Curve for Three-Legged Core Transformer 
The ratios of core loss " $\alpha$ " and " $\beta$ " were given in Section 6.1.3 and are repeated here for convinience: $\alpha=0.5245, \beta=0.4755$

Using the above results, the terms for separated core loss are represented:

$$
\begin{aligned}
R_{E} & =V^{2} /\left(P_{E} \cdot A \cdot L\right)=(V @ B=1.523)^{2} /\left[\beta \cdot\left(P_{C} @ B=1.523\right) \cdot(A \cdot L)\right] \\
& =(13800)^{2} /(0.4755 \cdot 46138) /(A \cdot L) \\
& =(8681) /(A \cdot L) \quad(\text { ohms })
\end{aligned}
$$

In Section 6.1.4, Equation (6.31) gives the DC hysteresis loss. From this core loss data, "aa" and "bb" are obtained as $a a=5165.7, b b=0.4596$.

The coercive force at each loop should be determined to meet the power loss $\mathrm{P}_{\mathrm{H}}$ at the $\mathrm{B}_{\mathrm{MAX}}$ given at the each loop. In case of Figure 6.30, "K" for Equation (6.35) is about 0.5 and $\mathrm{H}_{\mathrm{CTOP}}$ is about 2.2 A. The displacements at each $\mathrm{B}_{\mathrm{MAX}}$ are shown in Figure 6.31. The entire DC hysteresis loop is shown in Figure 6.32.

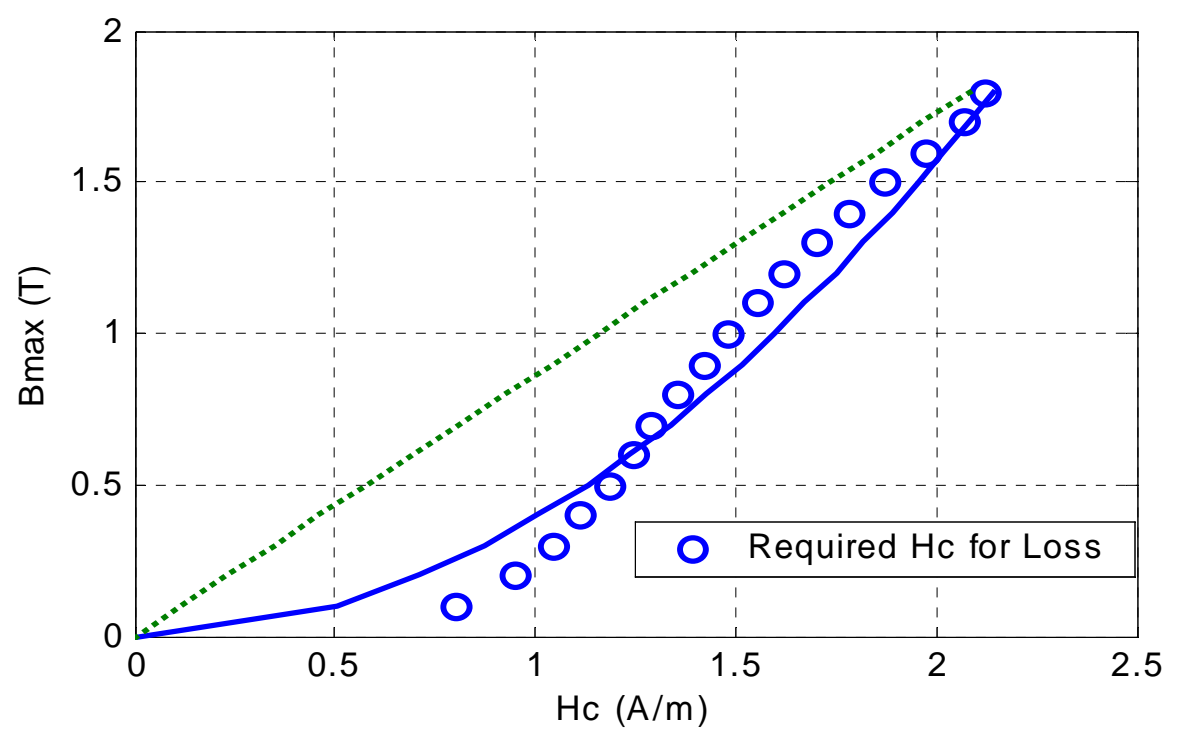

Figure 6.30 $\quad \mathrm{H}_{\mathrm{C}}$ and $\mathrm{B}_{\max }($ dotted line=linear, bold line=square root $)$ 


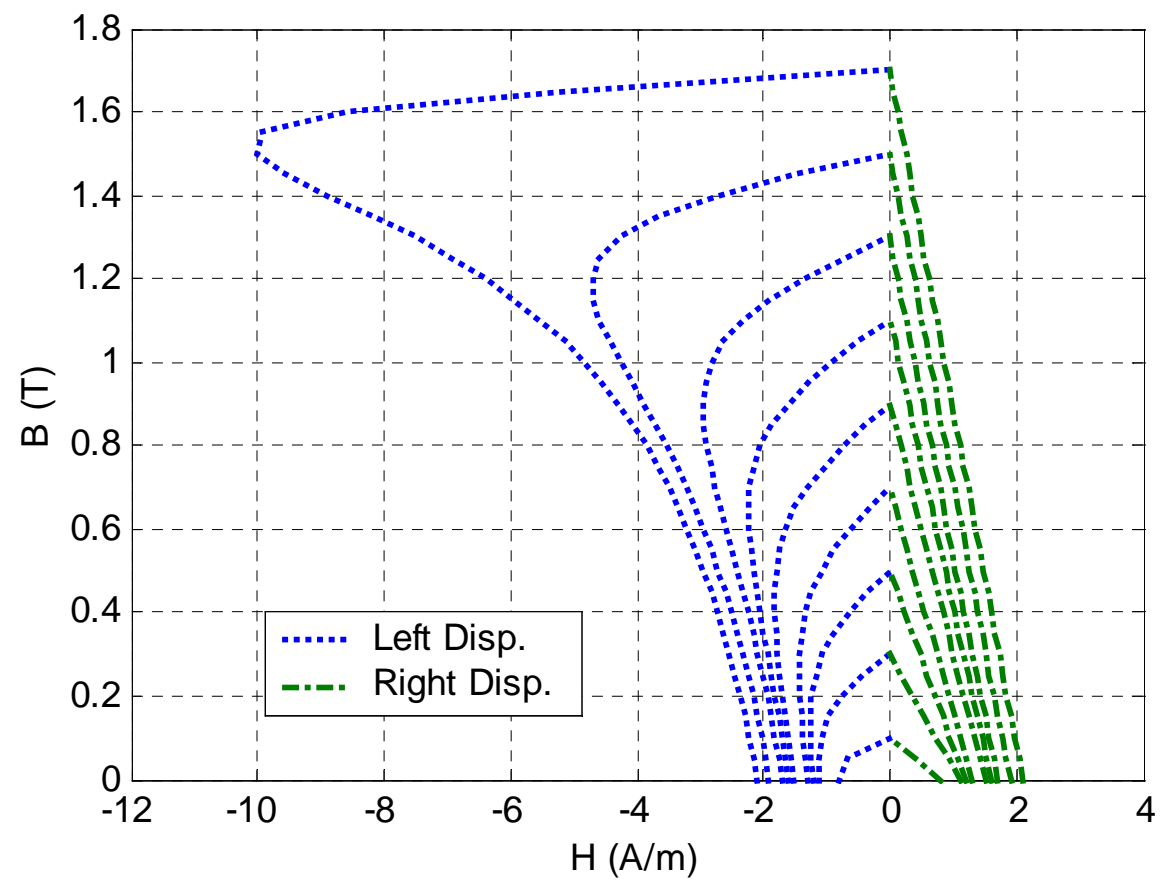

Figure 6.31 Left and Right Displacements of Resistive Hysteresis Current

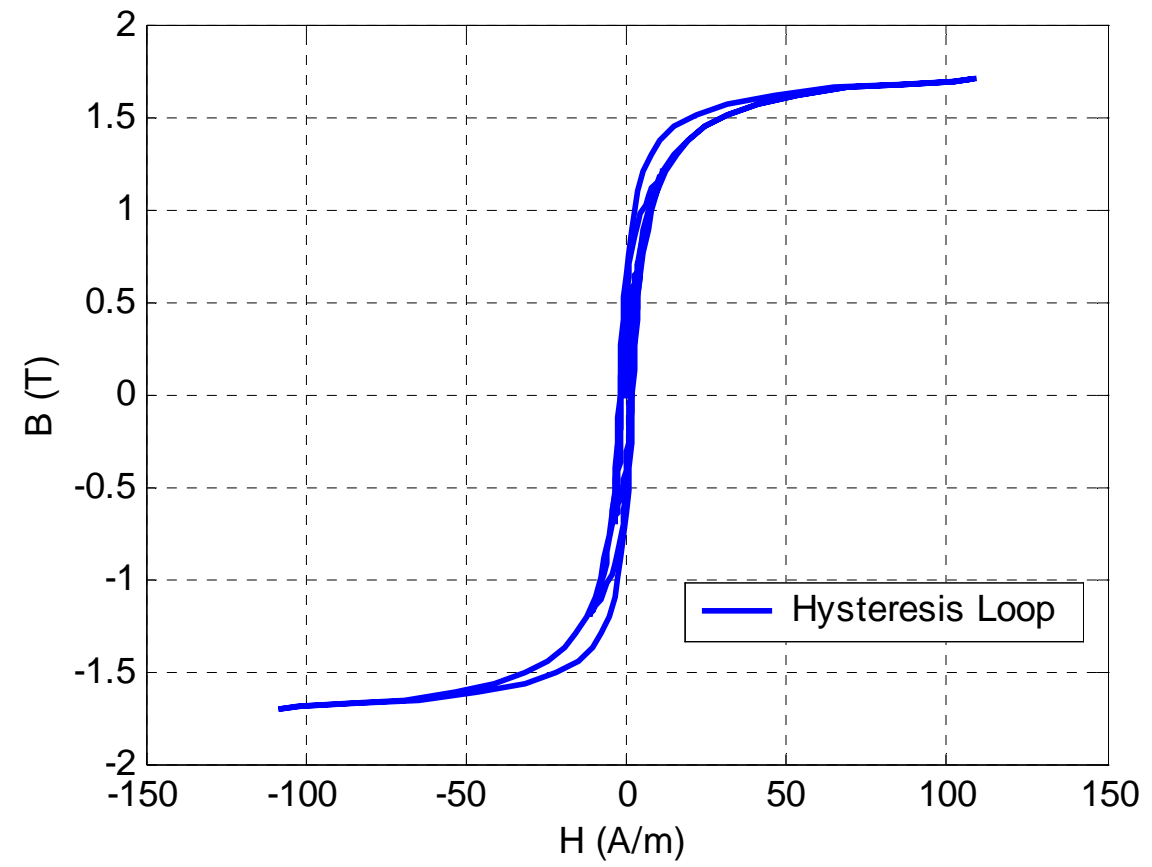

Figure 6.32 DC Hysteresis Loop Generated by the Model 


\subsubsection{ATP Implementation of Overall Transformer Model}

The overall transformer model for ATP implementation is shown in Figure 6.33. The core model, frequency-dependent coil resistance, and winding capacitances developed in Chapter 4 are included in ATP format.

Figure 6.34 shows the DC hysteresis loop modeled using a Type-60 current source controlled by TACS. Figure 6.35 shows the current of the eddy current loss and the resistive hysteresis current. Figure 6.36 shows the magnetizing current modeled with a Type-93 nonlinear inductance. Figures 6.37 and 6.38 show the line-current and windingcurrent waveforms.

After all models were implemented and run with ATP, the results of open-circuit and short-circuit simulations shown in Table 6.4 are very close to the test report.

Table 6.4 Comparisons with Test Report

\begin{tabular}{|c|c|}
\hline Test Report & Simulated Results \\
\hline \multicolumn{2}{|c|}{ Excitation Current @ 13.8kV Line } \\
\hline 94.12 A RMS @100\%Voltage & 96.7 A RMS $@ 100 \%$ Voltage \\
\hline $211.76 \mathrm{~A}_{\mathrm{RMS}} @ 110 \%$ Voltage & $208.9 \mathrm{~A}_{\mathrm{RMS}} @ 110 \%$ Voltage \\
\hline \multicolumn{2}{|c|}{ No Load Loss } \\
\hline 297.6 kW@100\%Voltage & 309.7 kW @100\%Voltage \\
\hline 402.24 kW@110\%Voltage & 359.6 kW@110\%Voltage \\
\hline \multicolumn{2}{|c|}{ Short-Circuit Current } \\
\hline $495.3 \mathrm{~A}_{\mathrm{RMS}} @ \mathrm{P}-\mathrm{S}$ & $495.3 \mathrm{~A}_{\mathrm{RMS}} @ \mathrm{P}-\mathrm{S}$ \\
\hline $128.9 \mathrm{~A}_{\mathrm{RMS}} @ \mathrm{P}-\mathrm{T}$ & $128.9 \mathrm{~A}_{\mathrm{RMS}} @ \mathrm{P}-\mathrm{T}$ \\
\hline $367.7 \mathrm{~A}_{\mathrm{RMS}} @ \mathrm{~S}-\mathrm{T}$ & $367.7 \mathrm{~A}_{\mathrm{RMS}} @ \mathrm{~S}-\mathrm{T}$ \\
\hline
\end{tabular}




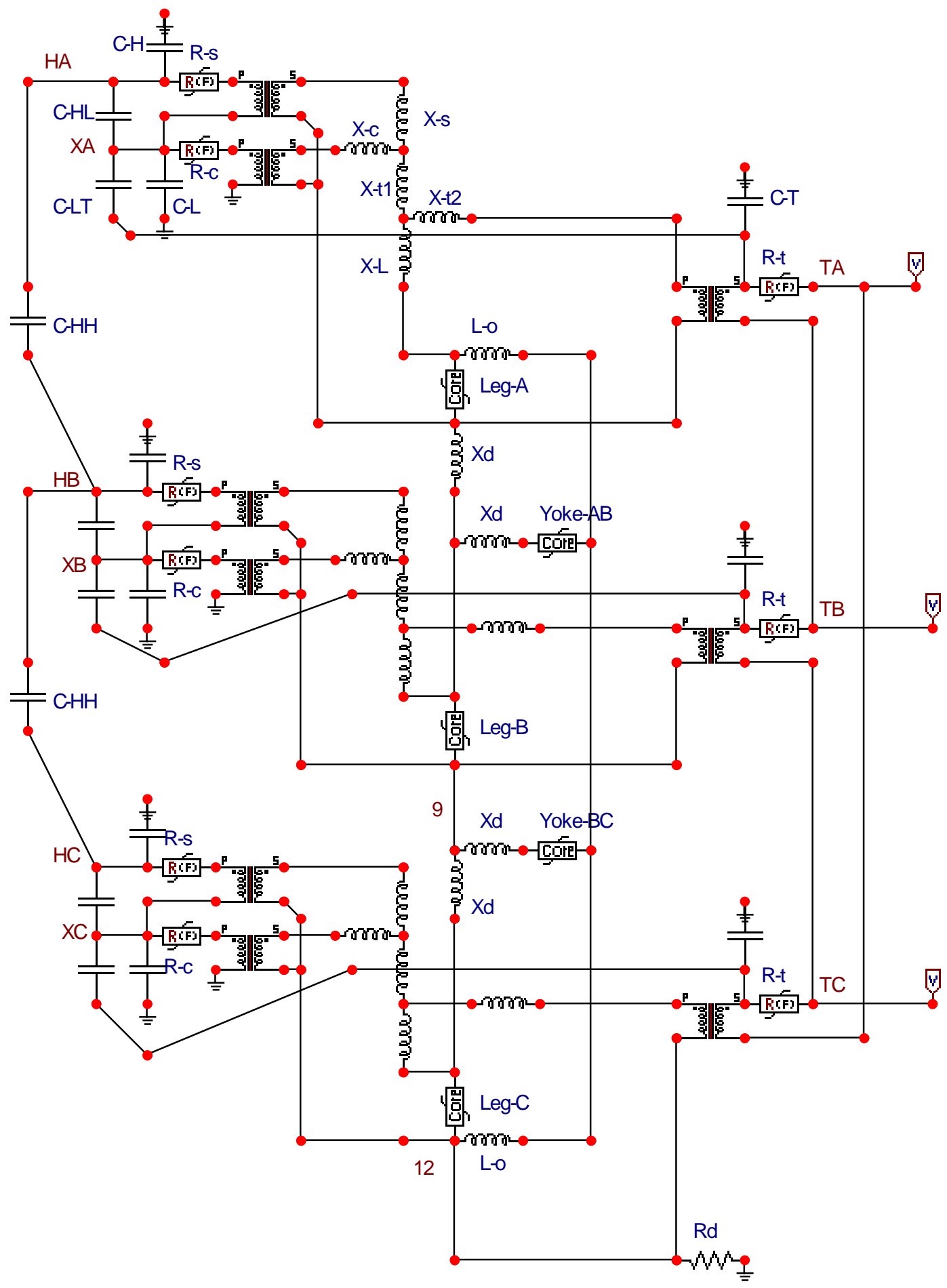

Figure 6.33 Equivalent Circuit for Three-legged Core Transformer, Implemented in ATP 


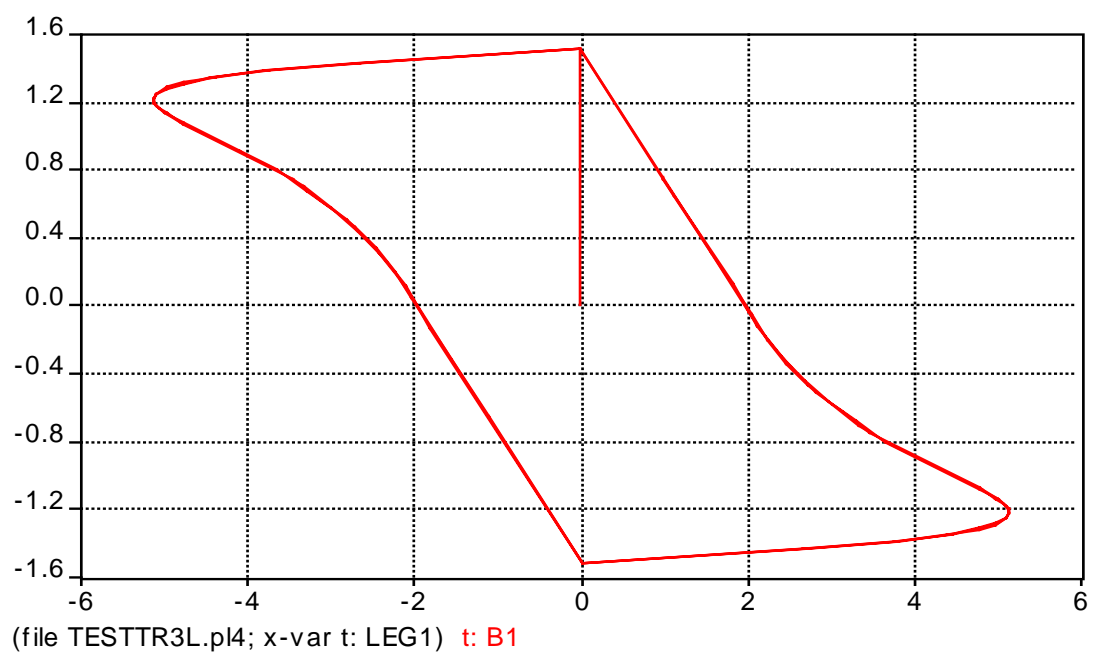

Figure 6.34 DC Hysteresis Loop Generated by ATP

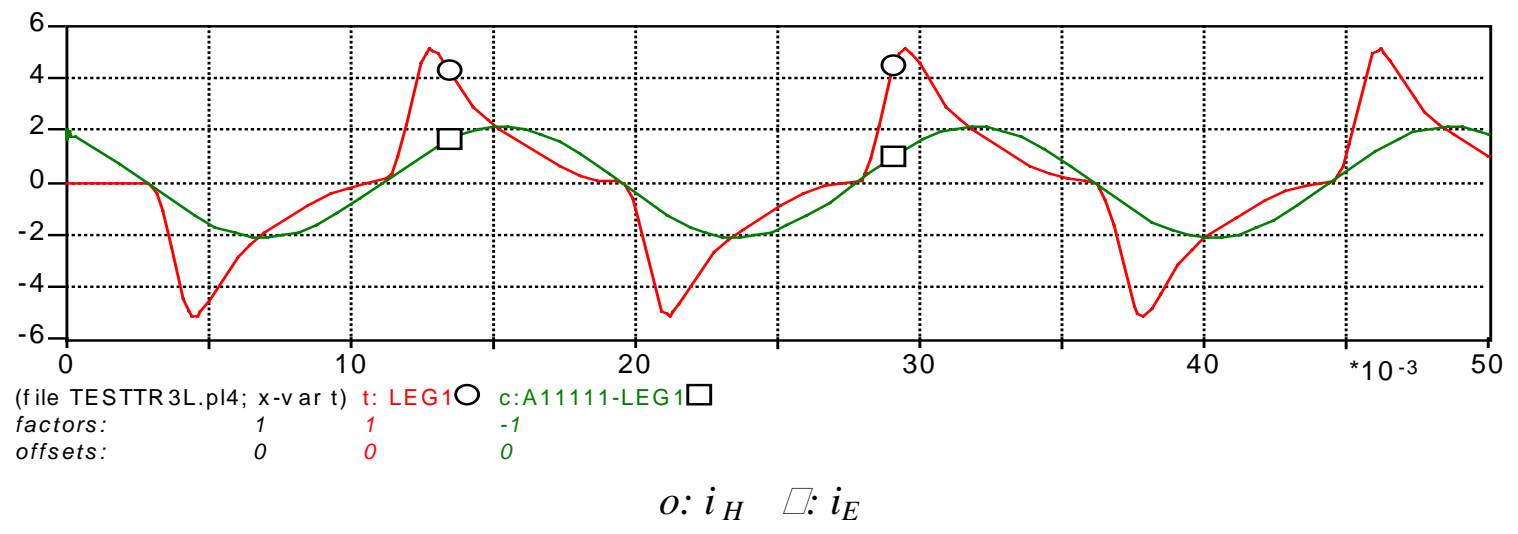

Figure 6.35 Eddy Current $\left(\mathrm{i}_{\mathrm{E}}\right)$ and Hysteresis Current $\left(\mathrm{i}_{\mathrm{H}}\right)$ Waveforms at $100 \% \mathrm{~V}$

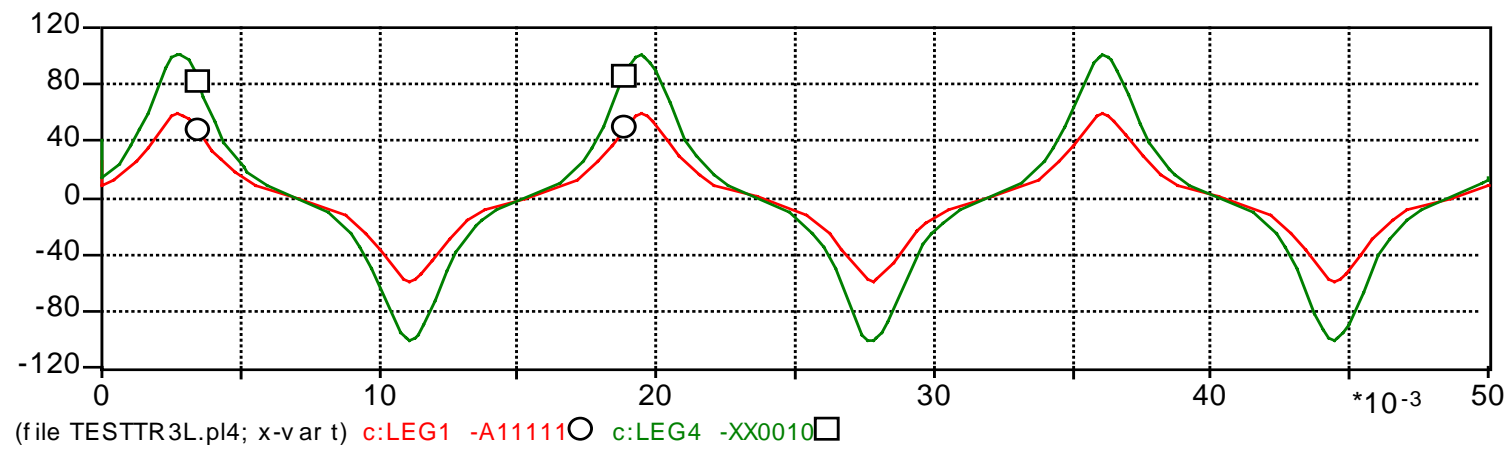

o: Leg-1 $\square$ : Yoke-AB

Figure 6.36 Mangnetizing Current Waveforms of Leg 1 and Yoke A-B at100\%V 


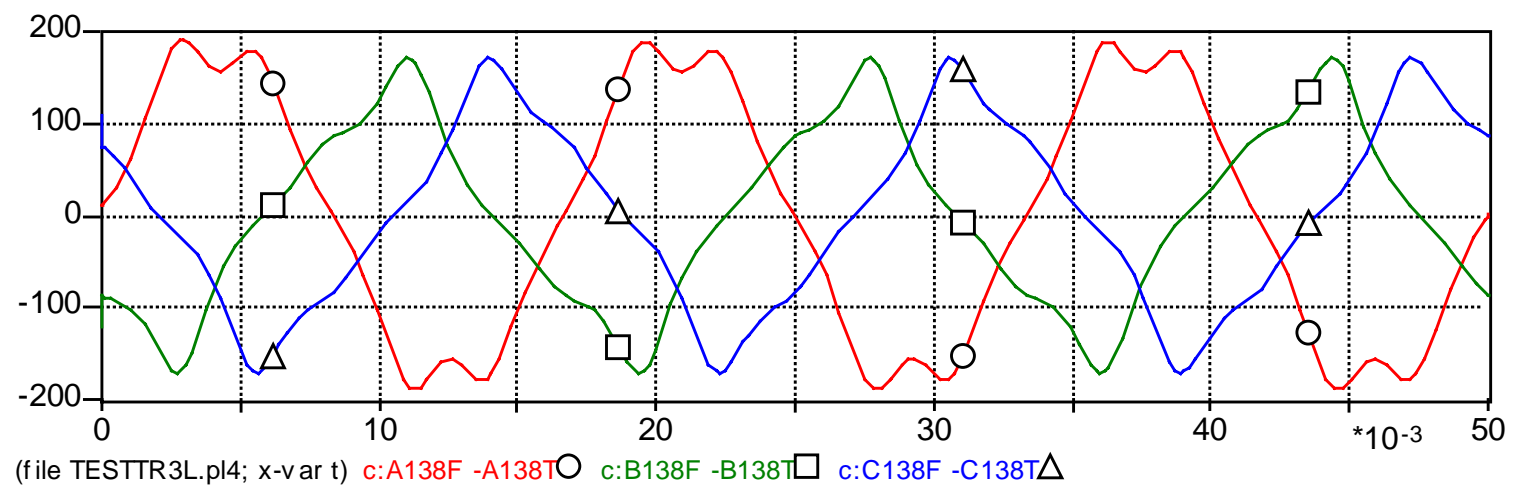

o: Line-A $\quad \square$ : Line-B $\quad \Delta$ : Line-C

Figure 6.37 Line Current Waveforms for Tertiary at 100\% Voltage

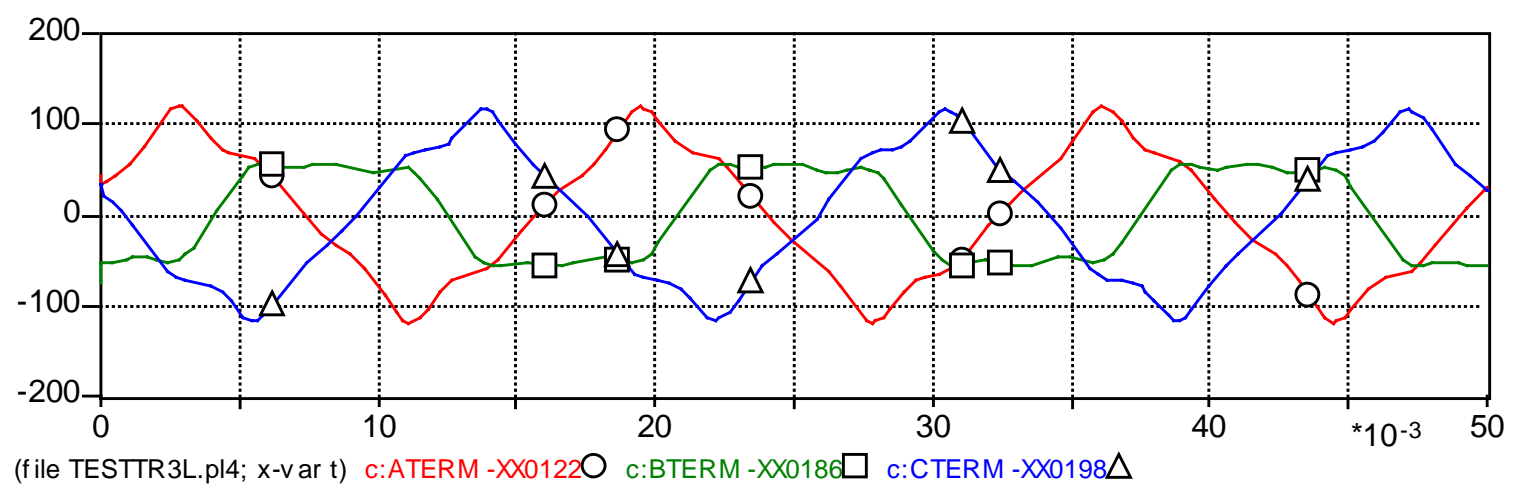

o: Phase-A $\square$ : Phase-B $\Delta$ : Phase-C

Figure 6.38 Winding Current Waveforms for Tertiary at 100\% Voltage 


\subsection{Shell-form Transformer}

\subsubsection{Leakage Inductance}

Shell-type transformers generally use pancake type windings. Upon closer examination of the magnetic model in Figure 6.39, leakage reluctance paths are almost the same as those of concentric winding transformer. Thus, the duality-based electrical equivalent circuit is the same as in Figure 6.2. The original leakage inductances need to be broken down into parts as was done in Figure 6.3.

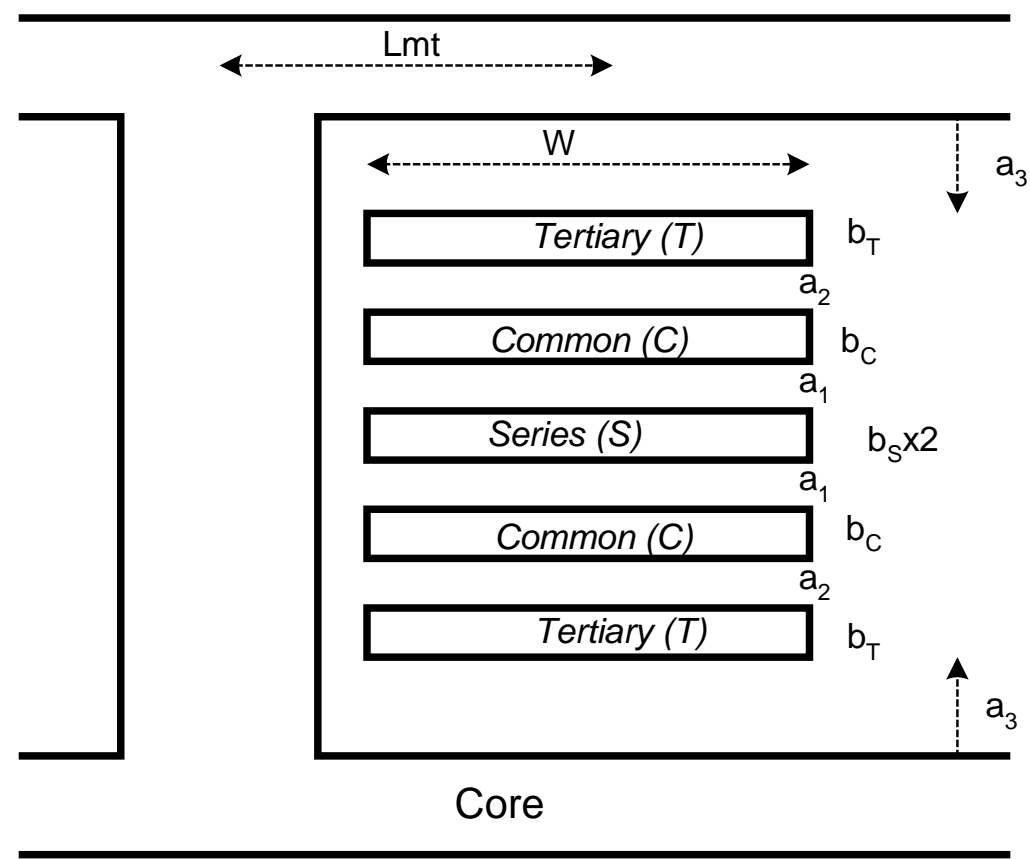

Figure 6.39 Cross-Section with Main Leakage Paths for Pancake type Winding

Equations (6.43) through (6.45) give leakage reactances based on physical dimensions [43]. Table 6.1 gives an example for winding width calculated based on DC resistance of coil. From Table 6.1, " $\mathrm{b}_{\mathrm{T}}$ " and "a $\mathrm{a}_{2}$ " in Equation (6.44) are generally smaller than " $\mathrm{b}_{\mathrm{S}}$ " and " $\mathrm{a}_{1}$ " in Equation (6.43). Therefore, $\mathrm{X}_{\mathrm{CT}}$ from Equation (6.44) is expected to 
be smaller than $\mathrm{X}_{\mathrm{SC}}$ from Equation (6.43). However, $\mathrm{X}_{\mathrm{CT}}$ is larger than $\mathrm{X}_{\mathrm{SC}}$ on the test data. In this case, the common winding or series winding need to be separated as shown in Figure 6.40.

$$
\begin{aligned}
& X_{S C}=\frac{\mu_{o} 2 \pi f N^{2} \cdot L m t \cdot\left(\frac{b_{C}+b_{S}}{3}+a_{1}\right)}{2 \cdot W} \\
& X_{C T}=\frac{\mu_{o} 2 \pi f N^{2} \cdot L m t \cdot\left(\frac{b_{C}+b_{T}}{3}+a_{2}\right)}{2 \cdot W} \\
& X_{S T}=\frac{\mu_{o} 2 \pi f N^{2} \cdot L m t \cdot\left(\frac{b_{S}+b_{T}}{3}+a_{1}+a_{2}+b_{C}\right)}{2 \cdot W}
\end{aligned}
$$

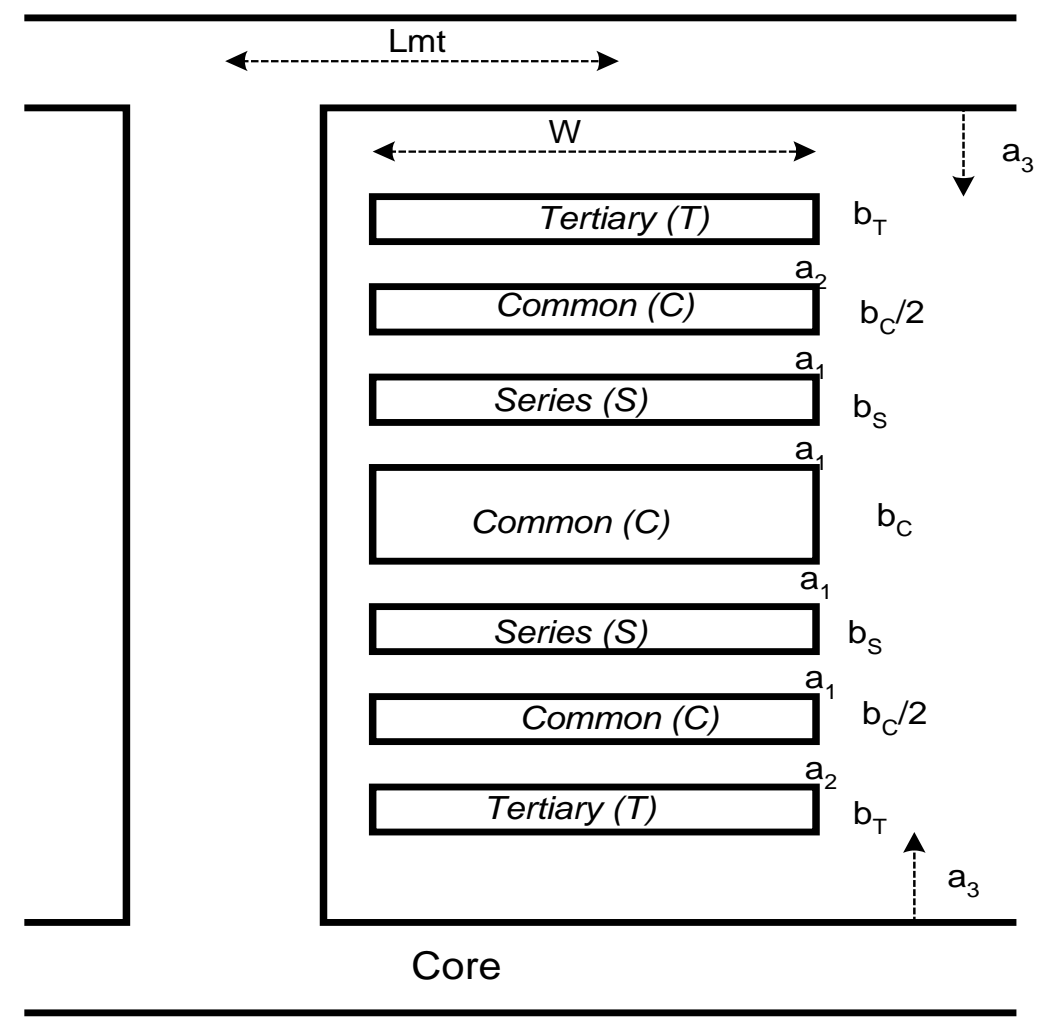

Figure 6.40 Cross-Section with Main Leakage Paths for Pancake type Winding 
Equations (6.46) through (6.48) give leakage reactances based on the physical dimensions in Figure 6.40. These equations have five unknowns. For the duality-based model shown in Figure 6.2, parameter estimations or some assumptions are necessary. The summation of the model parameters should be identical to the given short-circuit test data.

From Table 6.1, the ratios for winding width or winding area can be assumed from $R_{D C}$ and the ratios between $b_{S}, b_{C}$ and $b_{T}$ can be obtained as $b_{S}=7.53 \cdot b_{T}, b_{C}=7.82 \cdot b_{T}$. From voltage ratio, ratios for air gaps $a_{1}, a_{2}$ and $a_{3}$ can be assumed as $a_{1}=14.43 \cdot a_{3}$, $a_{2}=4.93 \cdot a_{3}$. Now, there are two unknowns for Equations (6.46) through (6.48). The least square fitting technique gives two values minimizing the differences and the thickness of coil and air gap. The values are approximately $b_{C}=1.332, b_{T}=0.171, b_{S}=1.286, a_{1}=0.117$, $\mathrm{a}_{2}=0.042$, and $\mathrm{a}_{3}=0.008$ from Equations (6.46) through (6.48). From these and Equation (6.49) through (6.54), the necessary reactance in Figures 6.2 and 6.3 can be obtained. Table 6.5 gives each reactance value.

$$
\begin{aligned}
& X_{S C}=\frac{\mu_{o} 2 \pi f N^{2} \cdot L m t \cdot \frac{1}{2} \cdot\left(\frac{b_{C}+b_{S}}{6}+a_{1}\right)}{2 \cdot W} \\
& X_{C T}=\frac{\mu_{o} 2 \pi f N^{2} \cdot L m t \cdot\left(\frac{b_{C}+b_{T}}{3}+a_{2}+\frac{a_{1}}{2}+\frac{b_{S}}{4}\right)}{2 \cdot W} \\
& X_{S T}=\frac{\mu_{o} 2 \pi f N^{2} \cdot L m t \cdot\left(\frac{b_{S}+b_{T}}{3}+a_{1}+a_{2}+\frac{b_{C}}{2}\right)}{2 \cdot W} \\
& X_{C}=\frac{X_{S C}+X_{C T}-X_{S T}}{2}=\frac{\mu_{o} 2 \pi f N^{2} \cdot L m t \cdot\left(\frac{-b_{C}}{12}\right)}{2 \cdot W}
\end{aligned}
$$




$$
\begin{aligned}
& X_{S}=\frac{X_{T S}+X_{S C}-X_{C T}}{2} \\
& X_{T}=X_{T-1}+X_{T-2}=\frac{X_{C T}+X_{T S}-X_{S C}}{2} \\
& X_{T-2}=\frac{\mu_{o} 2 \pi f N^{2} \cdot \mathrm{Lmt} \cdot\left(-b_{T} / 6\right)}{2 \cdot W} \\
& X_{4}=-3 \times X_{T-2} \\
& X_{5}=\frac{\mu_{o} 2 \pi f N^{2} \cdot \mathrm{Lmt} \cdot\left(a_{3}\right)}{2 \cdot \mathrm{W}}
\end{aligned}
$$

\begin{tabular}{|c|c|c|c|c|c|}
\hline & $\mathrm{S}-\mathrm{C}$ & C-T & $\mathrm{S}-\mathrm{T}$ & \multicolumn{2}{|c|}{ T-Core } \\
\hline Series $(\mathrm{S})$ & \multirow{2}{*}{$\begin{array}{c}0.3882 \\
\left(X_{S}\right)\end{array}$} & & \multirow{2}{*}{$\begin{array}{c}0.3882 \\
\left(\mathrm{X}_{\mathrm{S}}\right)\end{array}$} & & \\
\hline $\operatorname{Duct}\left(\mathrm{a}_{1}\right)$ & & & & & \\
\hline Common (C) & $\begin{array}{c}-0.1113 \\
\left(X_{C}\right)\end{array}$ & $\begin{array}{c}-0.1113 \\
\left(X_{C}\right)\end{array}$ & & & \\
\hline Duct $\left(a_{2}\right)$ & & $\begin{array}{c}0.9519 \\
\left(\mathrm{X}_{\mathrm{T}-1}\right)\end{array}$ & $\begin{array}{c}0.9519 \\
\left(\mathrm{X}_{\mathrm{T}-1}\right)\end{array}$ & & \\
\hline \multirow[t]{2}{*}{ Tertiary (T) } & & $\begin{array}{c}-0.0285 \\
\left(\mathrm{X}_{\mathrm{T}-2}\right) \\
\end{array}$ & $\begin{array}{c}-0.0285 \\
\left(\mathrm{X}_{\mathrm{T}-2}\right) \\
\end{array}$ & $\begin{array}{r}-0.0 \\
(\mathrm{X}\end{array}$ & $\begin{array}{l}285 \\
\Gamma-2)\end{array}$ \\
\hline & & & & $\begin{array}{c}0.0854 \\
\left(\mathrm{X}_{4}\right)\end{array}$ & \multirow{2}{*}{$\begin{array}{c}0.0862 \\
\left(X_{L}\right)\end{array}$} \\
\hline $\operatorname{Duct}\left(\mathrm{a}_{3}\right)$ & & & & $\begin{array}{c}0.0008 \\
\left(X_{5}\right)\end{array}$ & \\
\hline Total & $\begin{array}{c}0.2769 \\
\left(\mathrm{X}_{\mathrm{SC}}\right) \\
\end{array}$ & $\begin{array}{c}0.8126 \\
\left(\mathrm{X}_{\mathrm{CT}}\right)\end{array}$ & $\begin{array}{c}1.3121 \\
\left(\mathrm{X}_{\mathrm{ST}}\right) \\
\end{array}$ & \multicolumn{2}{|c|}{$\begin{array}{c}0.0577 \\
\left(\mathrm{X}_{\mathrm{TL}}\right)\end{array}$} \\
\hline Test Report & 0.27686 & 0.81259 & 1.3121 & \multicolumn{2}{|c|}{ N/A } \\
\hline
\end{tabular}

Table 6.5 Calculated Leakage Reactance in $\Omega$. 


\subsubsection{Core Saturation}

For a shell-form transformer, cross-sectional area ratios in Figure 6.41 were assumed on the basis that the flux density is the same for all paths. The portions of the core thus have the same conditions of saturation as the core inside the windings. Lengths were chosen on the basis that since most coil types are pancake type and then legs and yokes are longer than the limbs. Reluctances $\Re_{4} \sim \Re_{010}$ in Figure 6.42 represent the parallel combinations of two reluctances for upper and lower core sections.

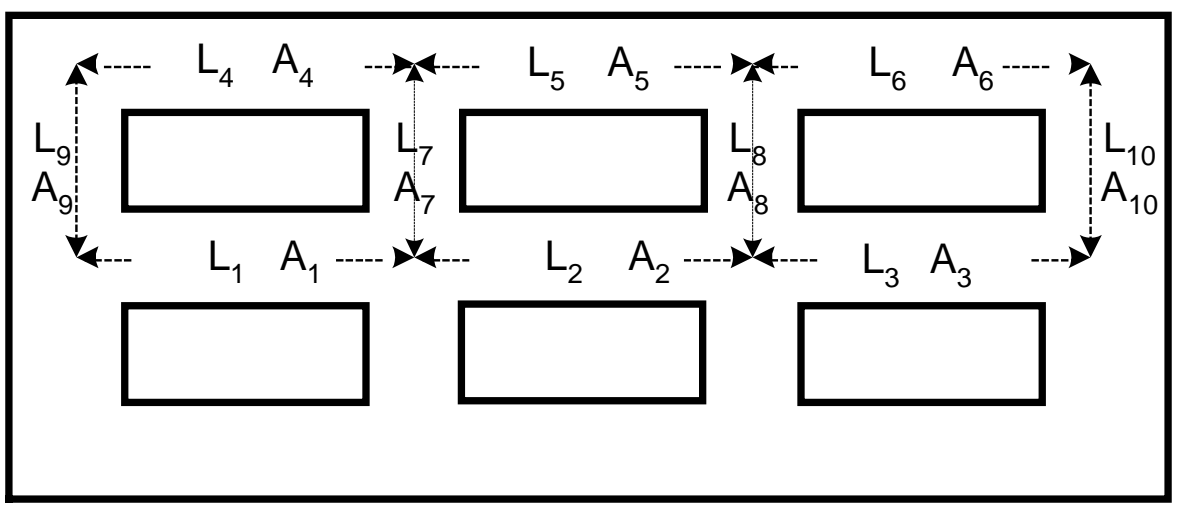

\begin{tabular}{|c|c|c|c|}
\hline \multicolumn{2}{|c|}{ Area ratio } & Length ratio \\
\hline \multicolumn{2}{|c|}{$A_{1}=A_{2}=A_{3}=1$} & $L_{1} \sim L_{6}=1$ \\
\hline Yoke $A_{4}=A_{5}=A_{6}=0.5$ & Middle Limb & Outer Limb & Limb \\
& $A_{7}=A_{8}=0.87$ & $A_{9}=A_{10}=0.5$ & $L_{7} \sim L_{10}=0.67$ \\
\hline
\end{tabular}

Notation: A: Area, L: Length,

1: Leg-A, 2:Leg-B, 3: Leg-C, 4:Outer Yoke, 5: Middle, 6: Outer-Yoke,

7: Middle Limb, 8: Middle Limb, 9:Outer Limb, 10:Outer Limb

Figure 6.41 Dimension of Shell-form Transformer 


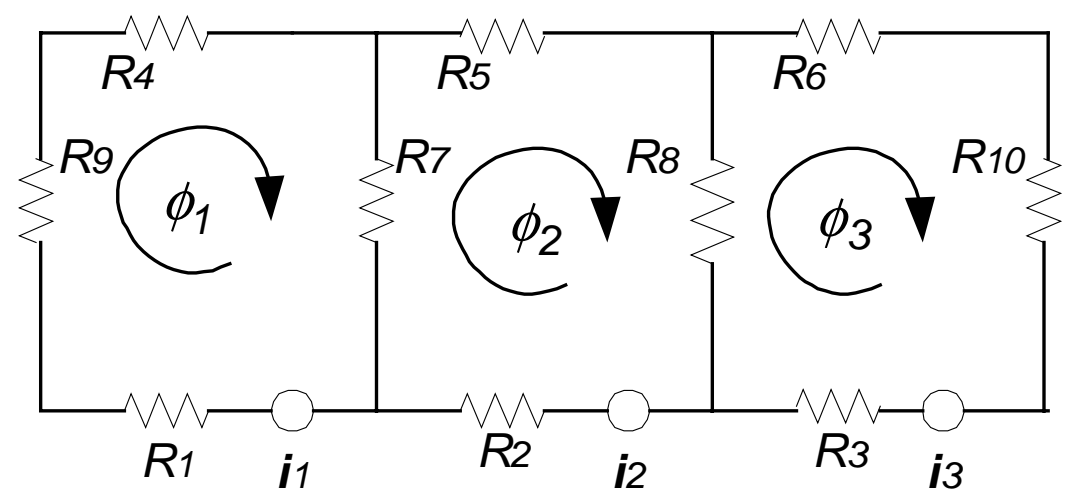

Figure 6.42 Magnetic Equivalent Circuit for Shell-form Transformer

$$
\begin{aligned}
& i_{1}=\phi_{1}\left(R_{1}+R_{4}+R_{9}\right)+R_{7}\left(\phi_{1}-\phi_{2}\right) \\
& i_{2}=\phi_{2}\left(R_{2}+R_{5}\right)+R_{7}\left(\phi_{2}-\phi_{1}\right)+R_{8}\left(\phi_{2}-\phi_{3}\right) \\
& i_{3}=\phi_{3}\left(R_{3}+R_{6}+R_{10}\right)+R_{8}\left(\phi_{3^{-}} \phi_{2}\right)
\end{aligned}
$$

where, $R_{1}=L_{1} /\left(\mu_{1} A_{1}\right), \mu_{1}=f\left(\phi_{1} / A_{1}\right), R_{2}=L_{2} /\left(\mu_{2} A_{2}\right), \mu_{2}=f\left(\phi_{2} / A_{2}\right)$, $R_{3}=L_{3} /\left(\mu_{3} A_{3}\right), \mu_{3}=f\left(\phi_{3} / A_{3}\right), R_{4}=L_{4} /\left(2 \mu_{4} A_{4}\right), \mu_{4}=f\left(\phi_{1} / 2 / A_{4}\right) \quad R_{5}=L_{5} /\left(2 \mu_{5} A_{5}\right)$, $\mu_{5}=f\left(\phi_{2} / 2 / A_{5}\right), R_{6}=L_{6} /\left(2 \mu_{6} A_{6}\right), \mu_{6}=f\left(\phi_{3} / 2 / A_{6}\right), R_{7}=L_{7} /\left(2 \mu_{7} A_{7}\right)$, $\mu_{7}=f\left(\left(\phi_{1}-\phi_{2}\right) / 2 / A_{7}\right), R_{8}=L_{8} /\left(2 \mu_{8} A_{8}\right), \mu_{8}=f\left(\left(\phi_{2}-\phi_{3}\right) / 2 / A_{8}\right), R_{9}=L_{9} /\left(2 \mu_{9} A_{9}\right)$, $\mu_{9}=f\left(\phi_{1} / 2 / A_{9}\right), R_{10}=L_{10} /\left(2 \mu_{10} A_{10}\right), \mu_{10}=f\left(\phi_{3} / 2 / A_{10}\right)$

In order to estimate the core dimensions, variables are classified as known and unknown.

Known values: $\phi_{1}=v_{1} /(\omega N), \phi_{2}=v_{2} /(\omega N), \phi_{3}=v_{3} /(\omega N)$ $v=$ peak-voltage for each phase, $\omega=2 \pi f, N=$ number of turn

Magnetizing Current: $I_{r m s, A V G}=\left(I_{1, r r m s}+I_{2, r r m s}+I_{3, \text { rrms }}\right) / 3$

Note: Real component of $I_{E X}$ has been removed, as explained in Section 4.2

Core dimenstions or ratios

Unknown values: $a, b$ for $\mu=\frac{B}{H}=\frac{(1-b \cdot B)}{a}$ 
If the core dimension ratios and average RMS magnetizing currents at $100 \%$ and $110 \%$ voltages are given, some optimization techinique can be used to estimate the a and b coefficients for the B-H Frolich equation from Equations (6.55) through (6.57).

The optimization performed is as follows: Details for waveform $i_{1}$ are given. Waveforms for $i_{2}$ and $i_{3}$ are obtained in a similar fashion based on Equations (6.56) and (6.57).

Minimize $f(a, b)=\left[\text { Measured } I_{A V G, R M S} @ 100 \% V-\text { Calculated } I_{A V G, R M S} @ 100 \% V\right]^{2}$

$$
+\left[\text { Measured } I_{A V G, R M S} @ 110 \% V-C a l c u l a t e d I_{A V G, R M S} @ 110 \% \mathrm{~V}\right]^{2}
$$

Subject to inequality constraints $0<a$ and $0<b<1$

Where, $\phi_{1}(k)=v_{1} /(\omega N) \times \sin (\pi \cdot k / 40)$ and $\phi_{2}(k)=v_{2} /(\omega N) \times \sin (\pi \cdot k / 40-2 \pi / 3)$

$$
\begin{aligned}
& \left.B_{1}(k)=B_{4}(k)=B_{9}(k)=\phi_{1}(k) / A_{1} \quad \text { and } B_{7}(k)=\left(\phi_{1}(k)-\phi_{2}(k)\right) / 2 / A_{7}\right) \\
& \mu_{1}(k)=\frac{1-b \cdot B_{1}(k)}{a} \text { and } \mu_{7}(k)=\frac{1-b \cdot B_{7}(k)}{a} \\
& R_{l}(k)=L_{1} /\left(\mu_{1}(k) \cdot A_{1}\right) \quad \text { and } \quad R_{7}(k)=L_{7} /\left(\mu_{7}(k) \cdot A_{7}\right) \\
& i_{1}(k)=\phi_{1}(k) \cdot\left(R_{1}(k)+R_{4}(k)+R_{9}(k)\right)+R_{7}(k) \cdot\left(\phi_{1}(k)-\phi_{2}(k)\right) \\
& I_{1, R M S}=\sqrt{\frac{\sum_{k=1}^{40}\left(i_{1}(k)\right)^{2}}{40}} \\
& I_{R M S, A V G}=\left(I_{1, R M S}+I_{2, R M S}+I_{3, R M S}\right) / 3
\end{aligned}
$$

From optimization technique (Appendix B.17) using Fmincon, the results are $a=3.7651, b=0.5651$. Figure 6.43 shows the obtained B-H curve. The calculated RMS currents for the three phases are $\left[\begin{array}{lll}99.9829 & 107.5387 & 107.5386\end{array}\right]$ A at $100 \%$ voltage. The calculated average RMS current is $105.02 \mathrm{~A}$ and the difference from the test report is only $7.9357 \times 10^{-5} \mathrm{~A}$. The calculated RMS currents for the three phases are $[215.8171$ $228.2594228 .2594] \mathrm{A}$ at $110 \%$ voltage. The calculated average RMS current is 224.11 A and the difference from the test report is $3.7518 \times 10^{-5} \mathrm{~A}$. 
Figure 6.44 shows the $\lambda$-i curve for each core section of the example transformer.

Figure 6.45 shows the current waveforms of lines at $100 \%$ voltage simulated using MATLAB.

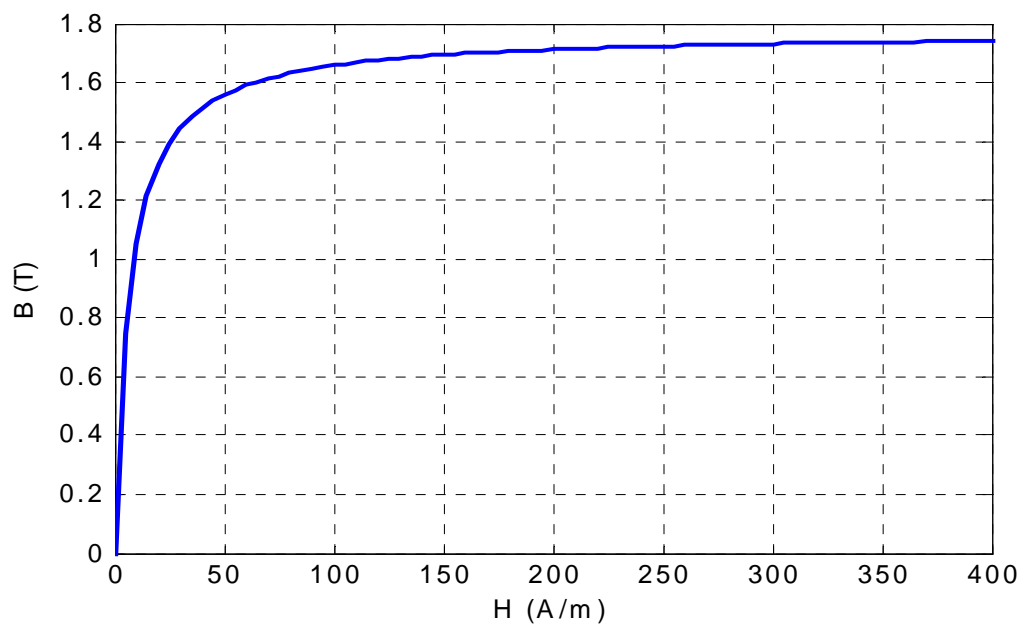

Figure 6.43 B-H for Each Section

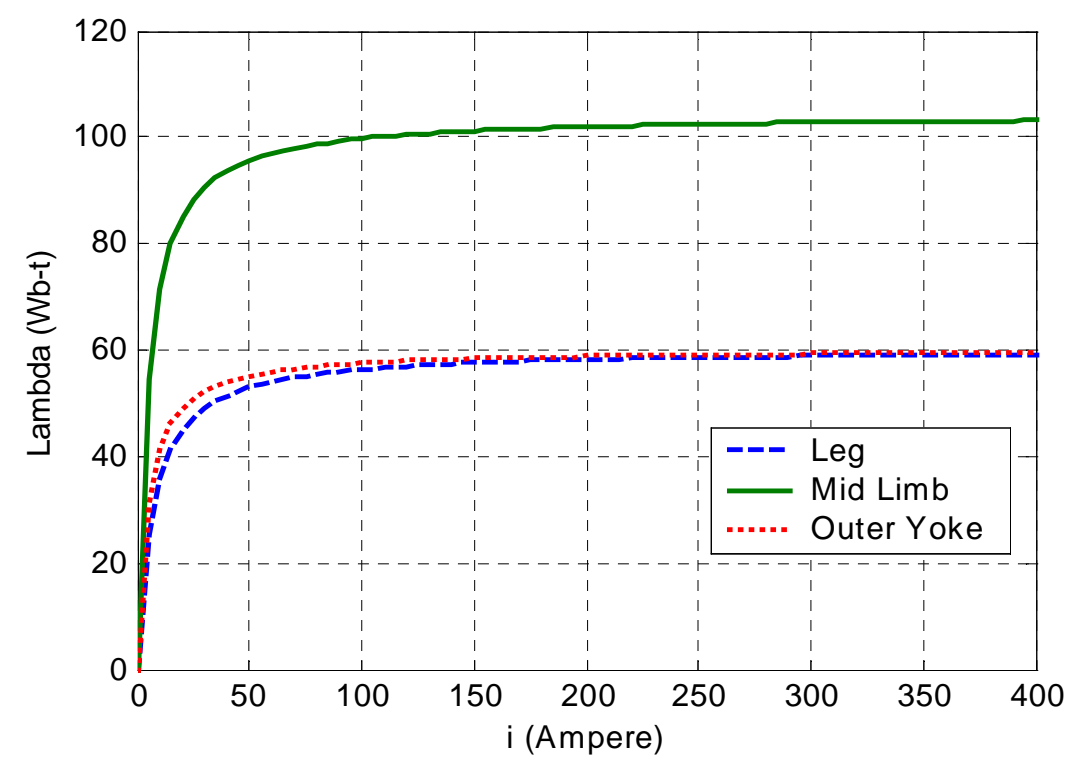

Figure 6.44 $\lambda-i$ Magnetization Curves for Each Section 


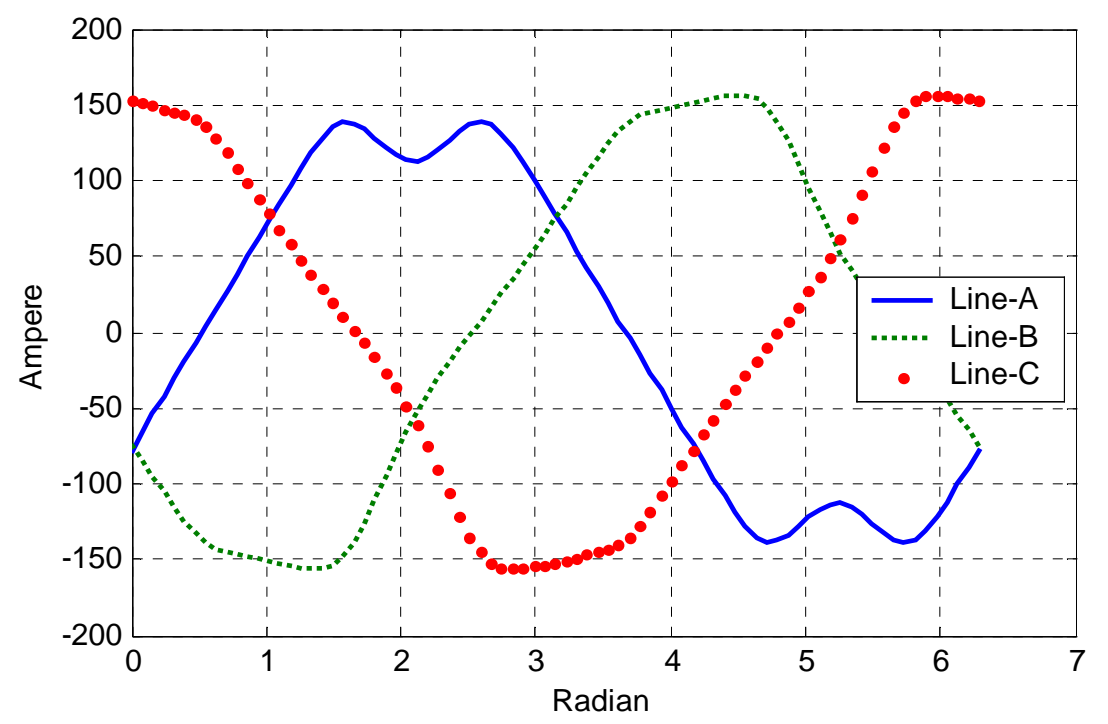

Figure 6.45 Current Waveforms for Each Line at 100\% Voltage

\subsubsection{Core Loss Model}

From test data, average core losses at $100 \%$ voltage and $110 \%$ voltage are given as $[297600,402240](\mathrm{W})$ at $\lambda=[51.77,56.95](\mathrm{Wb}-\mathrm{t})$.

The calculated average core loss should be:

$$
\sum_{n=1}^{10} P_{n}\left(B_{n}\right) \cdot A_{n} \cdot L_{n}=\sum_{n=1}^{10} \frac{a \cdot B_{n}}{1-b \cdot B_{n}} \cdot A_{n} \cdot L_{n}
$$

The optimization (Appendix B.19) performed is as follows:

Minimize $f(a, b)$

$$
\begin{gathered}
=\left[P @ 100 \% \mathrm{~V}-\sum_{n=1}^{10} \frac{a \cdot B_{n}}{1-b \cdot B_{n}} \cdot A_{n} \cdot L_{n}\right]^{2}+\left[P @ 110 \% \mathrm{~V}-\sum_{n=1}^{10} \frac{a \cdot B_{n}}{1-b \cdot B_{n}} \cdot A_{n} \cdot L_{n}\right]^{2}(6.60) \\
\text { Subject to inequality constraints } 0<\text { a and } 0<b<1
\end{gathered}
$$


The flux density B and normalized dimensions are also given as below from the previous section, but are repeated here for convinience.

\begin{tabular}{|c|c|c|c|c|c|c|c|c|c|c|}
\hline & $\begin{array}{c}\text { Leg- } \\
1\end{array}$ & $\begin{array}{c}\text { Leg- } \\
2\end{array}$ & $\begin{array}{c}\text { Leg- } \\
3\end{array}$ & $\begin{array}{c}\text { Yoke } \\
-1\end{array}$ & $\begin{array}{c}\text { Yoke } \\
-2\end{array}$ & $\begin{array}{c}\text { Yoke } \\
-3\end{array}$ & $\begin{array}{c}\text { Mid } \\
\text { Limb }\end{array}$ & $\begin{array}{c}\text { Mid } \\
\text { Limb }\end{array}$ & $\begin{array}{c}\text { Outer } \\
-1\end{array}$ & $\begin{array}{c}\text { Outer } \\
-2\end{array}$ \\
\hline Core No & 1 & 2 & 3 & 4 & 5 & 6 & 7 & 8 & 9 & 10 \\
\hline Area & 1 & 1 & 1 & 1 & 1 & 1 & 1.732 & 1.732 & 1 & 1 \\
\hline Length & 1 & 1 & 1 & 1 & 1 & 1 & .67 & .67 & .67 & .67 \\
\hline $\begin{array}{c}\text { B } \\
@ 100 \% V\end{array}$ & 1.523 & 1.523 & 1.523 & 1.523 & 1.523 & 1.523 & 1.523 & 1.523 & 1.523 & 1.523 \\
\hline $\begin{array}{c}\text { B } \\
@ 110 \% V\end{array}$ & 1.675 & 1.675 & 1.675 & 1.675 & 1.675 & 1.675 & 1.675 & 1.675 & 1.675 & 1.675 \\
\hline
\end{tabular}

Thus, $\mathrm{a}=7071.8$ and $\mathrm{b}=0.4272$ are calculated for the core saturation curve by using optimization technique Fmincon and Equation (6.60). In this case, there is only a minor difference $[0.01520 .0112] \mathrm{W}$ between the given core loss and the calculated closs. Figure 6.46 shows the core loss curve for the shell-form transformer.

The core loss ratios " $\alpha$ " and " $\beta$ " were given in Section 6.1.3 and are repeated here for convinience: $\alpha=0.5245, \beta=0.4755$

Using the above result, the terms for separated core loss are represented:

$$
\begin{aligned}
R_{E} & =V^{2} /\left(P_{E} \cdot A \cdot L\right)=(V @ B=1.523)^{2} /\left[b \cdot\left(P_{C} @ B=1.523\right) \cdot(A \cdot L)\right] \\
& =(13800)^{2} /(0.4755 \cdot 30828) /(A \cdot L) \\
& =(12992) /(A \cdot L) \quad(\Omega)
\end{aligned}
$$

In Section 6.1.4, the equation for DC hysteresis loss was given as Equation (6.31). From the core loss separation and Equation (6.31), "aa" and "bb" are obtained as $a a=$ $3448.9, b b=0.4596$. 


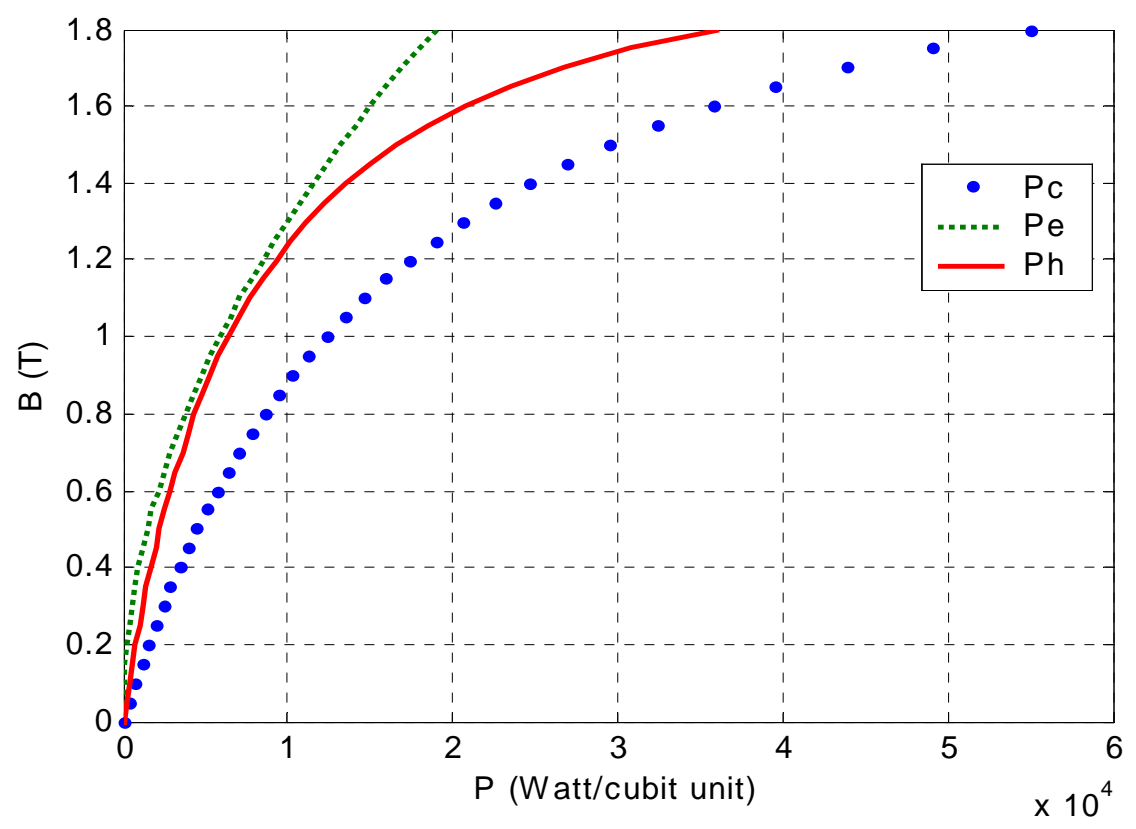

Figure 6.46 Core Loss Curve using Frolich Equation for Shell-form Transformer

The coercive force at each loop should be determined to meet the power loss $\mathrm{P}_{\mathrm{H}}$ at the $\mathrm{B}_{\max }$ given for each loop. In case of Figure 6.47, " $\mathrm{K}$ " for Equation (6.35) is about 0.5 and $\mathrm{H}_{\text {ctop }}$ is about $1.4 \mathrm{~A}$. The displacements at each $\mathrm{B}_{\max }$ are shown in Figure 6.48. The entire DC hysteresis loop is shown in Figure 6.49. 


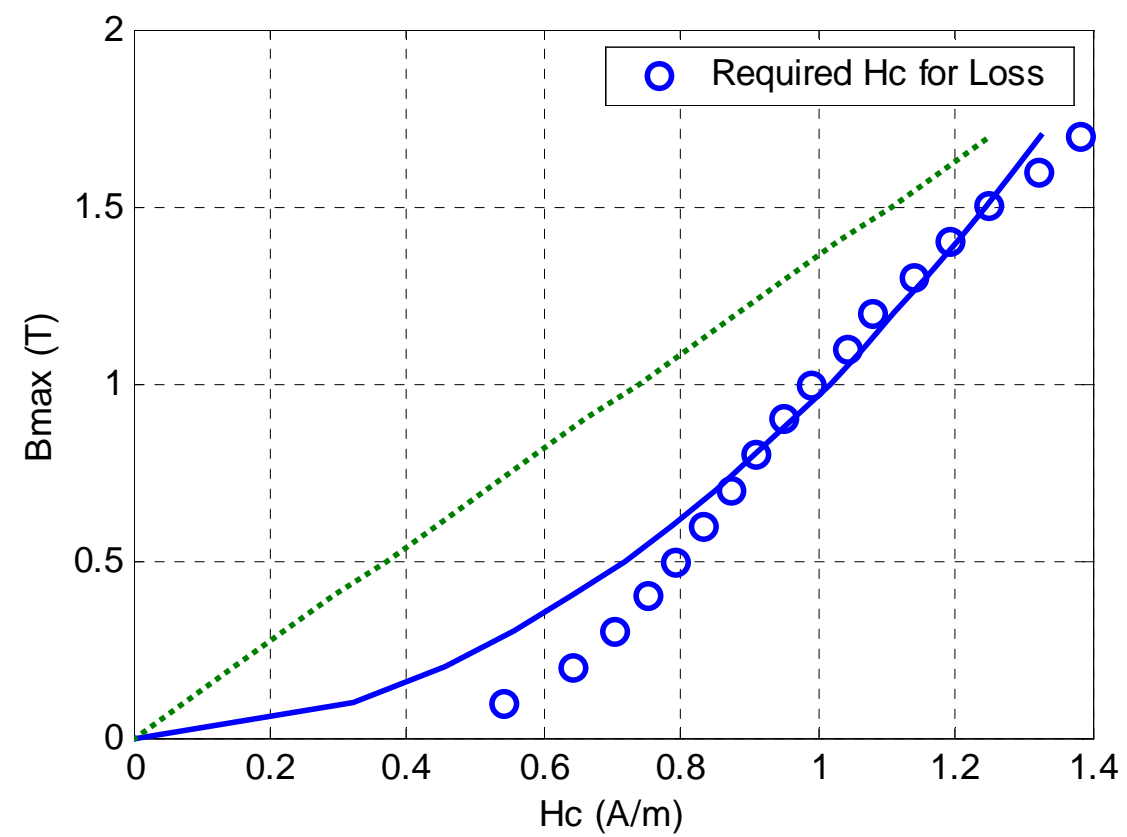

Figure 6.47 $\quad \mathrm{H}_{\mathrm{C}}$ and $\mathrm{B}_{\max }($ dotted line=linear, bold line=square root $)$

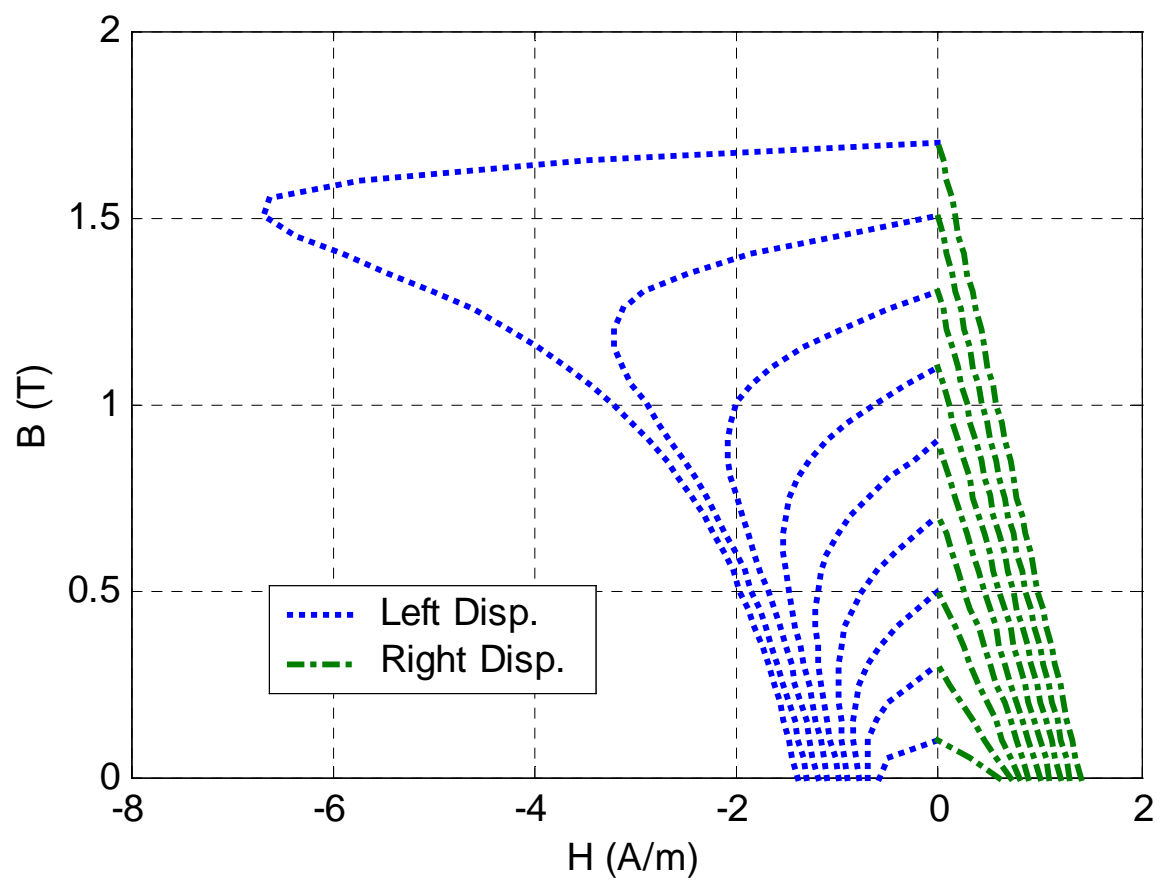

Figure 6.48 Left and Right Displacements of Resistive Hysteresis Current 


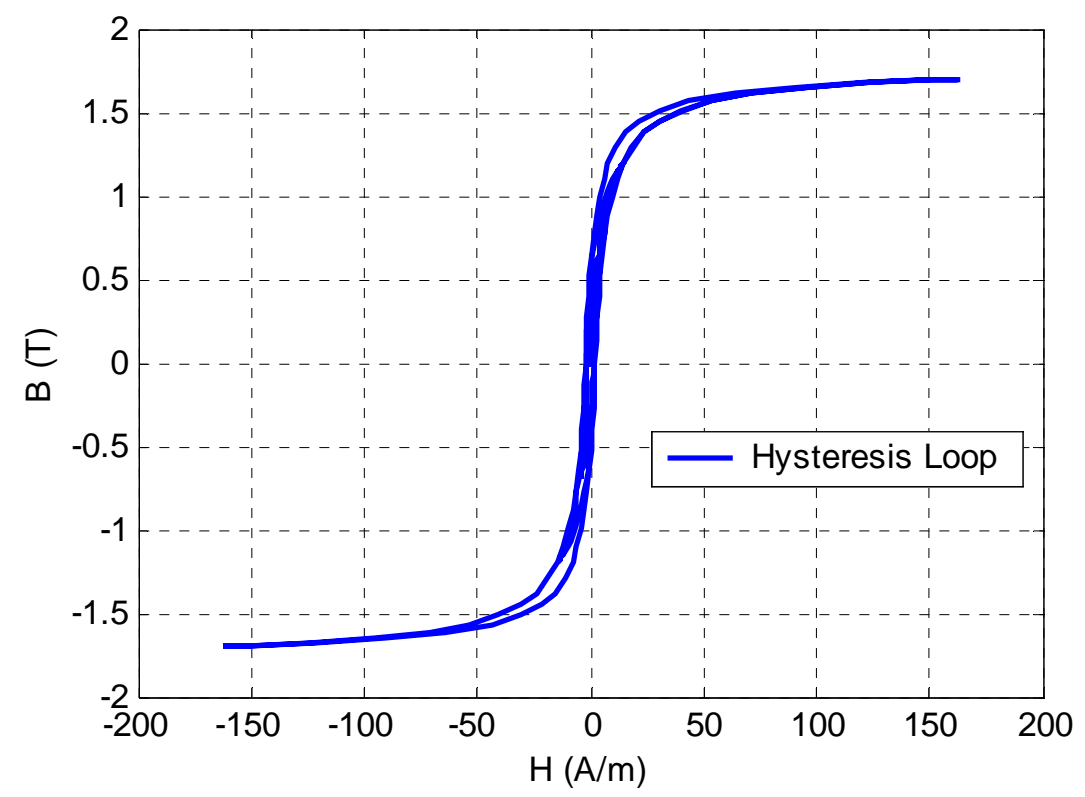

Figure 6.49 DC Hysteresis Loop Generated by the Model

\subsubsection{ATP Implementation of Overall Transformer Model}

The overall transformer model for ATP implementation is shown in Figure 6.50. The core model, frequency-dependent coil resistance and winding capacitances developed in Chapter 4 are included in ATP format.

Figure 6.51 shows the DC hysteresis loop modeled using a Type-60 current source controlled by TACS. Figure 6.52 shows the current of the eddy current loss and the resistive hysteresis current. Figure 6.53 shows the magnetizing current modeled with a Type-93 nonlinear inductance. Figures 6.54 and 6.55 show the line-current and windingcurrent waveforms. 


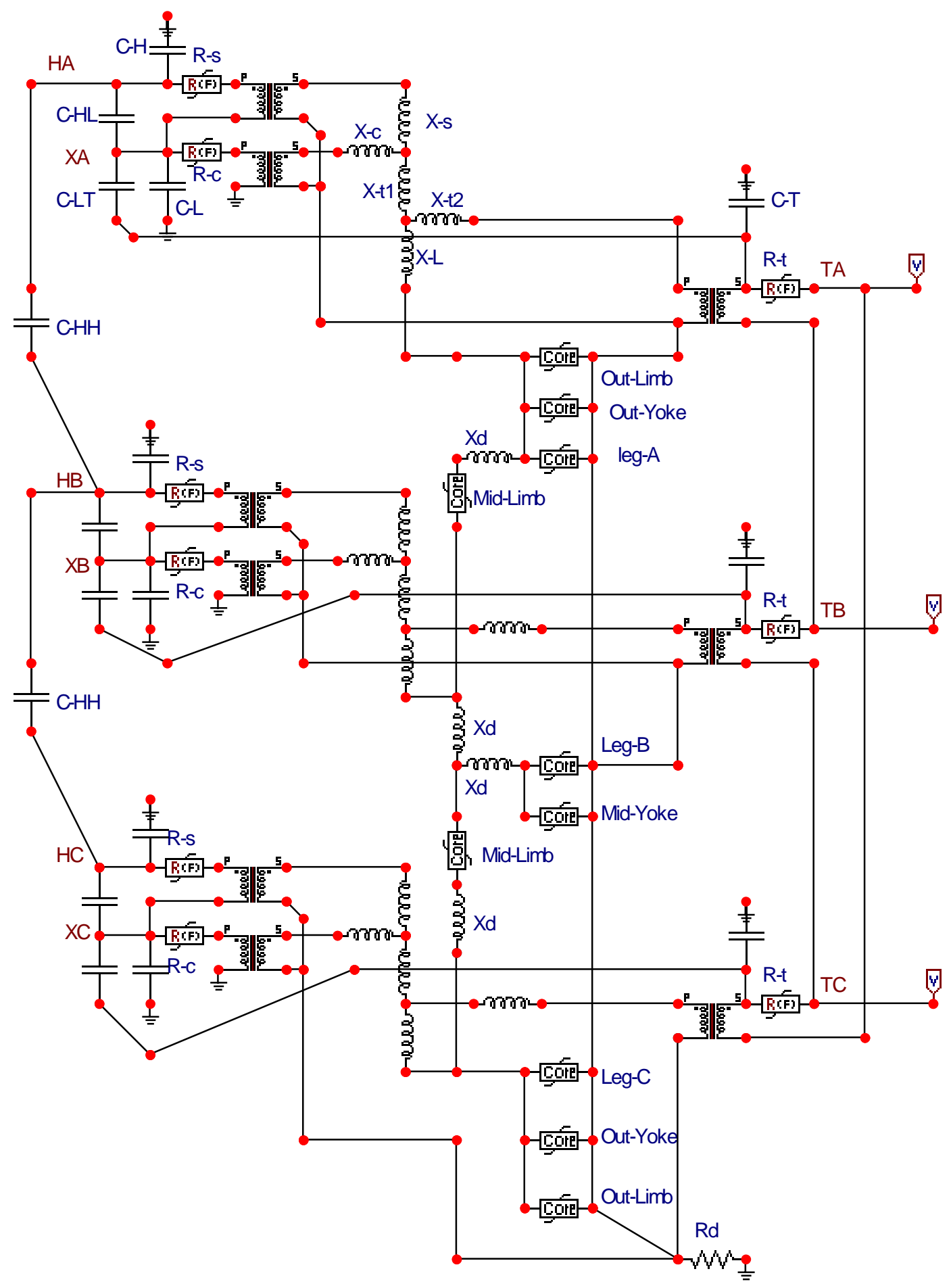

Figure 6.50 Equivalent Circuit for Sell-form Transformer, Implemented in ATP 


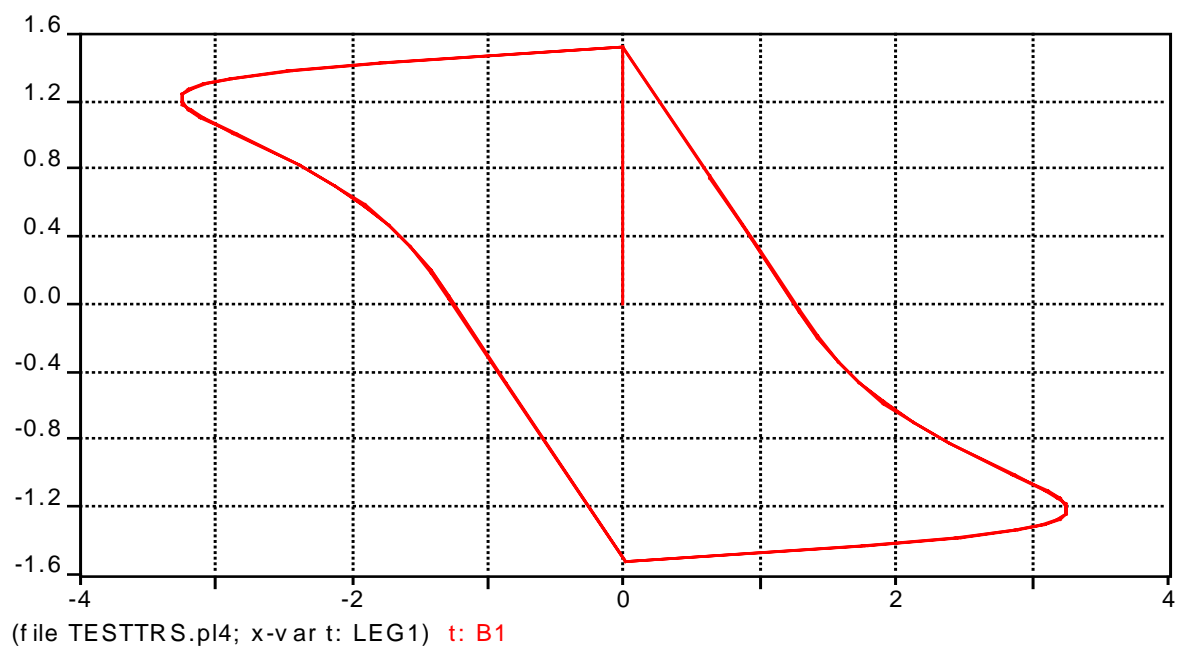

Figure 6.51 DC Hysteresis Loop Generated by ATP

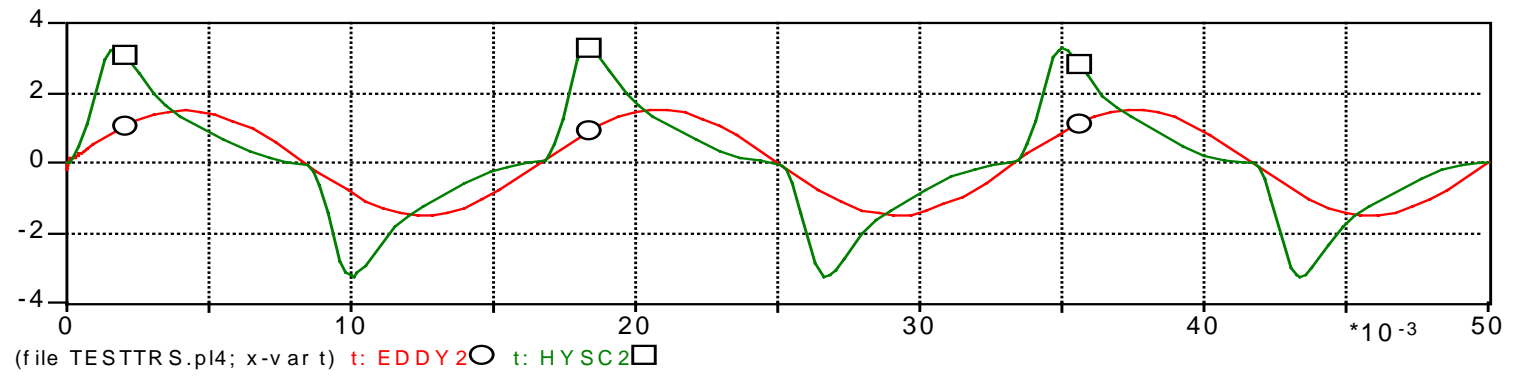

$o: i_{E} \quad \square: i_{H}$

Figure 6.52 Eddy Current $\left(i_{E}\right)$ and Hysteresis Current $\left(i_{H}\right)$ Waveforms at 100\%V

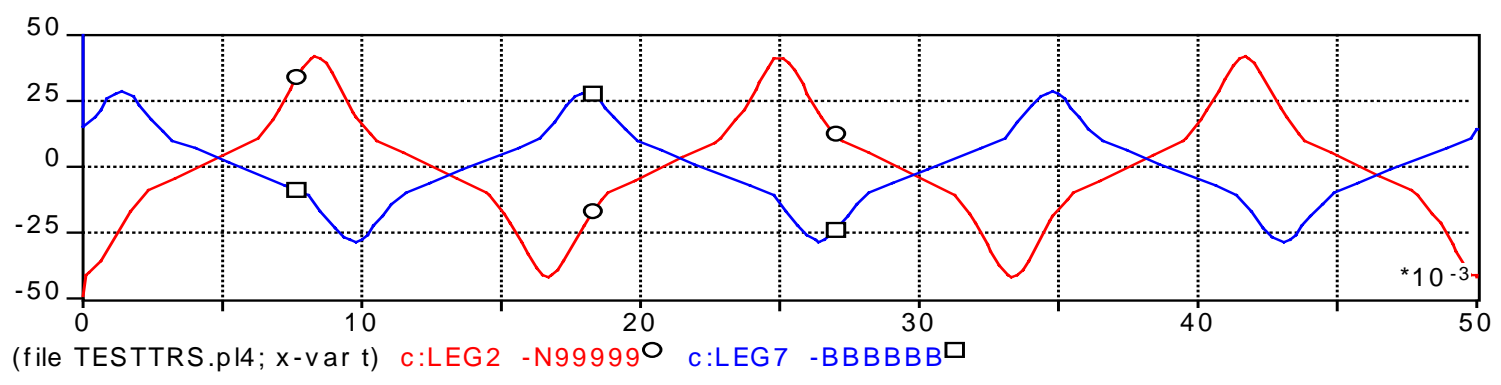

o: Leg-1 $\square$ : Mid Limb A-B at $100 \% \mathrm{~V}$

Figure 6.53 Mangnetizing Current Waveforms of Leg 2 and Mid Limb A-B (Leg-7) 


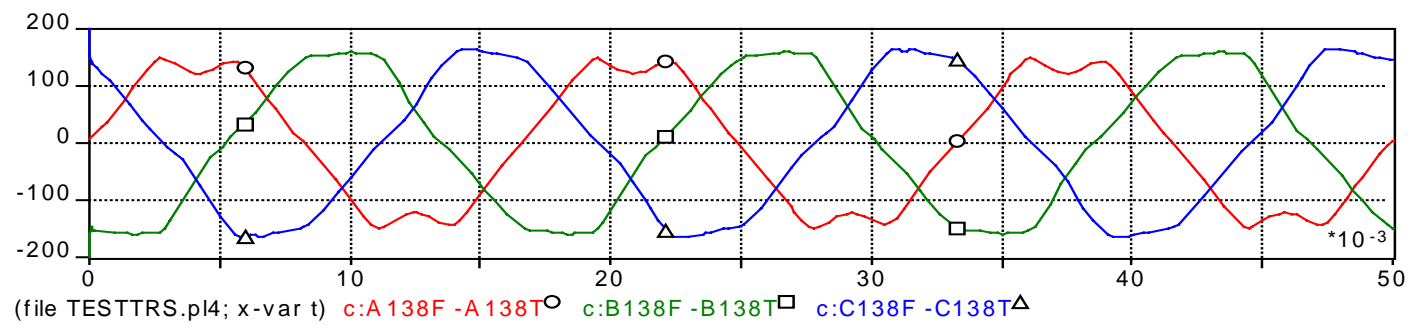

o: Line-A $\square$ : Line-B $\quad \Delta$ : Line-C

Figure 6.54 Line Current Waveforms for Tertiary at 100\% Voltage

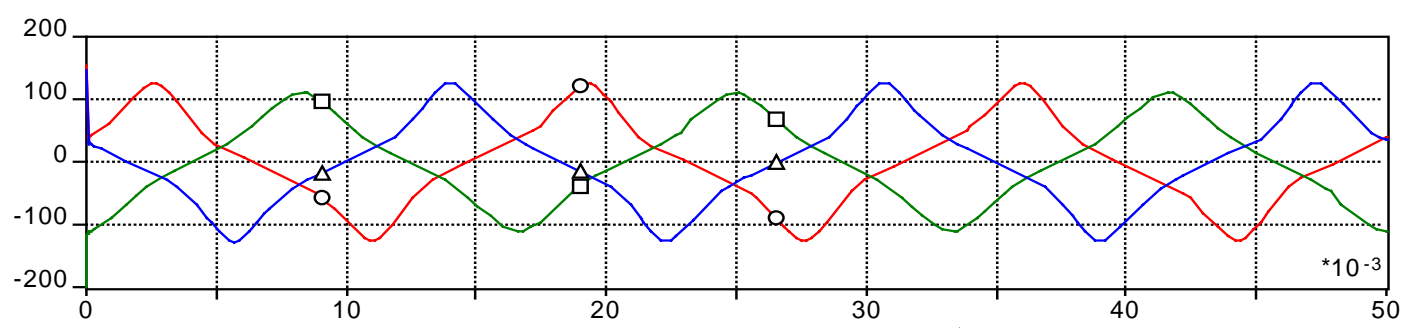

(file TESTTRS.pl4; $x$-var t) c:A138T-XX01030 c:B138T-B-T c:C138T-C-T $\Delta$

o: Phase-A $\square$ : Phase-B $\Delta$ : Phase-C

Figure 6.55 Winding Current Waveforms for Tertiary at 100\% Voltage

After all models were implemented and run with ATP, the results of open-circuit and short-circuit simulations shown in Table 6.6 are very close to the test report.

Table 6.6 Comparisons with Test Report

\begin{tabular}{|c|c|}
\hline Test Report & Simulated Results \\
\hline \multicolumn{2}{|c|}{ Excitation Current @ 13.8kV Line } \\
\hline 94.12 $\mathrm{A}_{\text {RMS }} @ 100 \%$ Voltage & $101.9 \mathrm{~A}_{\mathrm{RMS}} @ 100 \%$ Voltage \\
\hline $211.76 \mathrm{~A}_{\mathrm{RMS}} @ 110 \%$ Voltage & $223.4 \mathrm{~A}_{\mathrm{RMS}} @ 110 \%$ Voltage \\
\hline \multicolumn{2}{|c|}{ No Load Loss } \\
\hline $297.6 \mathrm{~kW} @ 100 \%$ Voltage & $283.4 \mathrm{~kW} @ 100 \%$ Voltage \\
\hline $402.24 \mathrm{~kW} @ 110 \%$ Voltage & $373.1 \mathrm{~kW} @ 110 \%$ Voltage \\
\hline \multicolumn{2}{|c|}{ Short-Circuit Current } \\
\hline $495.3 \mathrm{~A}_{\mathrm{RMS}} @ \mathrm{P}-\mathrm{S}$ & $495.3 \mathrm{~A}_{\mathrm{RMS}} @ \mathrm{P}-\mathrm{S}$ \\
\hline $128.9 \mathrm{~A}_{\mathrm{RMS}} @ \mathrm{P}-\mathrm{T}$ & $128.9 \mathrm{~A}_{\mathrm{RMS}} @ \mathrm{P}-\mathrm{T}$ \\
\hline $367.7 \mathrm{~A}_{\mathrm{RMS}} @ \mathrm{~S}-\mathrm{T}$ & $367.7 \mathrm{~A}_{\mathrm{RMS}} @ \mathrm{~S}-\mathrm{T}$ \\
\hline
\end{tabular}




\section{CHAPTER 7}

\section{SIMULATIONS FOR MODEL EVALUATION}

This chapter presents the results of the ATP simulations for benchmarking the developed models. Steady-state excitation, de-energization, and re-energization transients are simulated and compared to a BCTRAN-based model used in an earlier investigation [14]. The performance of the equivalent circuit and parameters are summarized. In addition, simulation results using models developed in Chapters 5 and 6 are compared to actual transients event records [14].

In this chapter, the core type of the example transformer is assumed as shell-form, since the manufacturer of the example transformer typically made shell-from transformers. The required data for transformer modeling are the basic factory test data and the estimated relative physical dimensions of the core.

\subsection{Comparison with BCTRAN Model}

Comparisons with the earlier BCTRAN Model are steady-state excitation, deenergization, and re-energization transients. The transformer of Table 3.2 is used for the comparison. 
In the case of the earlier BCTRAN model, core magnetization and losses were attached externally on the tertiary. The core was modeled as three sets of type-98 inductances in parallel with linear resistors connected in delta. Using the $100 \%$ and $110 \%$ excitation data from the factory test report, the RMS magnetizing current was obtained by removing the core loss component from the exciting current. This model ignores core structure and represents the transformer as essentially three single-phase transformers.

In the case of the duality model, core magnetization and losses are attached at the legs and yokes respectively. Each core section is modeled as a type-93 inductance in parallel with a type-60 TACS current source for hysteresis loss and eddy current loss. Here the shell-form autotransformer model first introduced in Section 6.3 is used. Although the factory test report gives only $100 \%$ and $110 \%$ excitation data, more $\lambda-i$ points can be obtained for the type-93 inductances from the core saturation function. All parameters were obtained by the procedures described in Chapters 4 and Section 6.3.

First, no-load steady-state excitation at $110 \%$ of the nominal voltage of $118-\mathrm{kV}$ is simulated. The core flux and current waveforms are presented in Figures 7.1 through 7.6.

In the case of the earlier BCTRAN-based model, only the $100 \%$ and $110 \%$ factory excitation data were used. Therefore, the shape of the $\lambda$-i curve is a simpler 2-segment piecewise linear curve, as can be seen in Figure 7.2. The core-loss current waveforms in Figure 7.3 are sinusoidal and the shape of $\lambda$ vs. core-loss current curve in Figure 7.4 is a circle, since it is modeled as a linear resistance. As seen in Figure 7.5, the hysteresis loss 
is actually dependent on flux linked, so this simplistic representation may give incorrect simulation results.

In the case of the new duality model, more $\lambda-i$ points can be obtained from the core saturation function and the shape of the $\lambda-i$ curve is smooth (Figure 7.2). Thus, the results can be more accurate. The hysteresis loss is also dependent on flux as seen in Figure 7.5.

For steady-state excitation, excitation currents are identical in each phase in the case of the BCTRAN model (Figure 7.6). However, line currents differ from phase to phase with the new duality model. The phase current waveforms for the outer legs are quite similar but differ from that of the center leg. This is due to consideration of the actual core structure.

At $30 \mathrm{~ms}$, the switches de-energize the transformer, with each phase being electrically interrupted when the current passes through zero. In this way, the residual fluxes are determined. No arc phenomena in the switch are considered. Results are shown in Figure 7.6. Phase "b" clears first after the mechanical disconnection. The two remaining phases are next interrupted. Residual flux remains in the core of the new duality model, as shown in Figures 7.7 and 7.8. The BCTRAN model has no residual flux, since the energy stored in its core is dissipated in its core loss resistance.

When the transformer with the residual flux is reconnected to the network (inrush), the residual flux at the instant acts as a DC offset to the sinusoidal flux linkage waveforms in Figure 7.9. This DC component may drive the core deep into the saturation region depending on the conditions. Hence the inrush currents are considerably increased 
in Figure 7.10. The inrush currents of the duality model are larger than those of BCTRAN model, since, the slope of saturation curve is low in the saturation region.

Although there was no benchmarking data available for these, they exhibit much more reasonable behaviors than those provided by the earlier BCTRAN-based model.
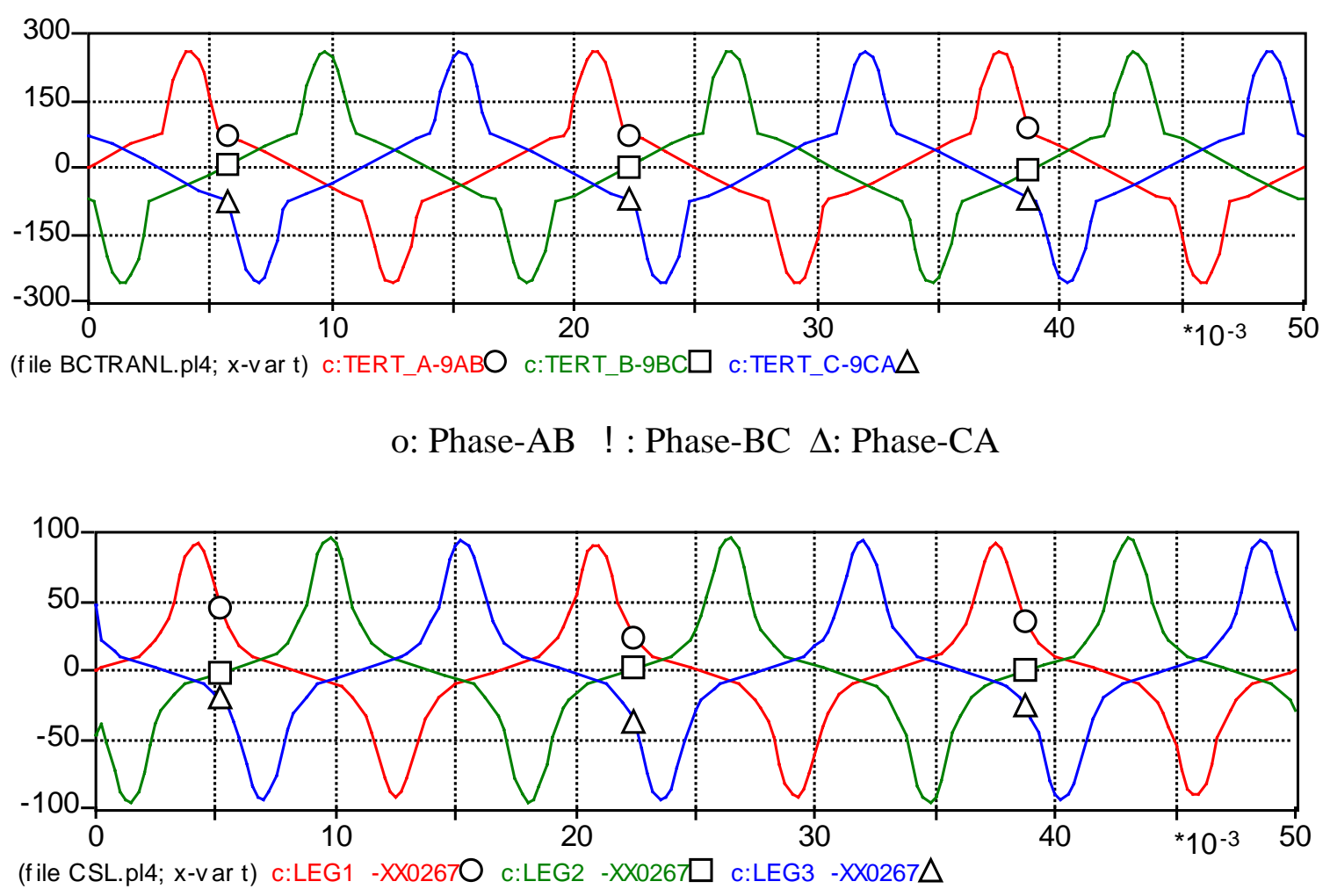

o: Leg-1 $\square$ : Leg-2 $\Delta$ : Leg-3

$\mathrm{X}$-axis: Time in Secs, Y-axis: Current in Amperes on $13.8-\mathrm{kV}$ base

BCTRAN Model (Top) and Duality Model (Bottom)

Figure 7.1 Transformer Magnetizing Current for Three Legs 

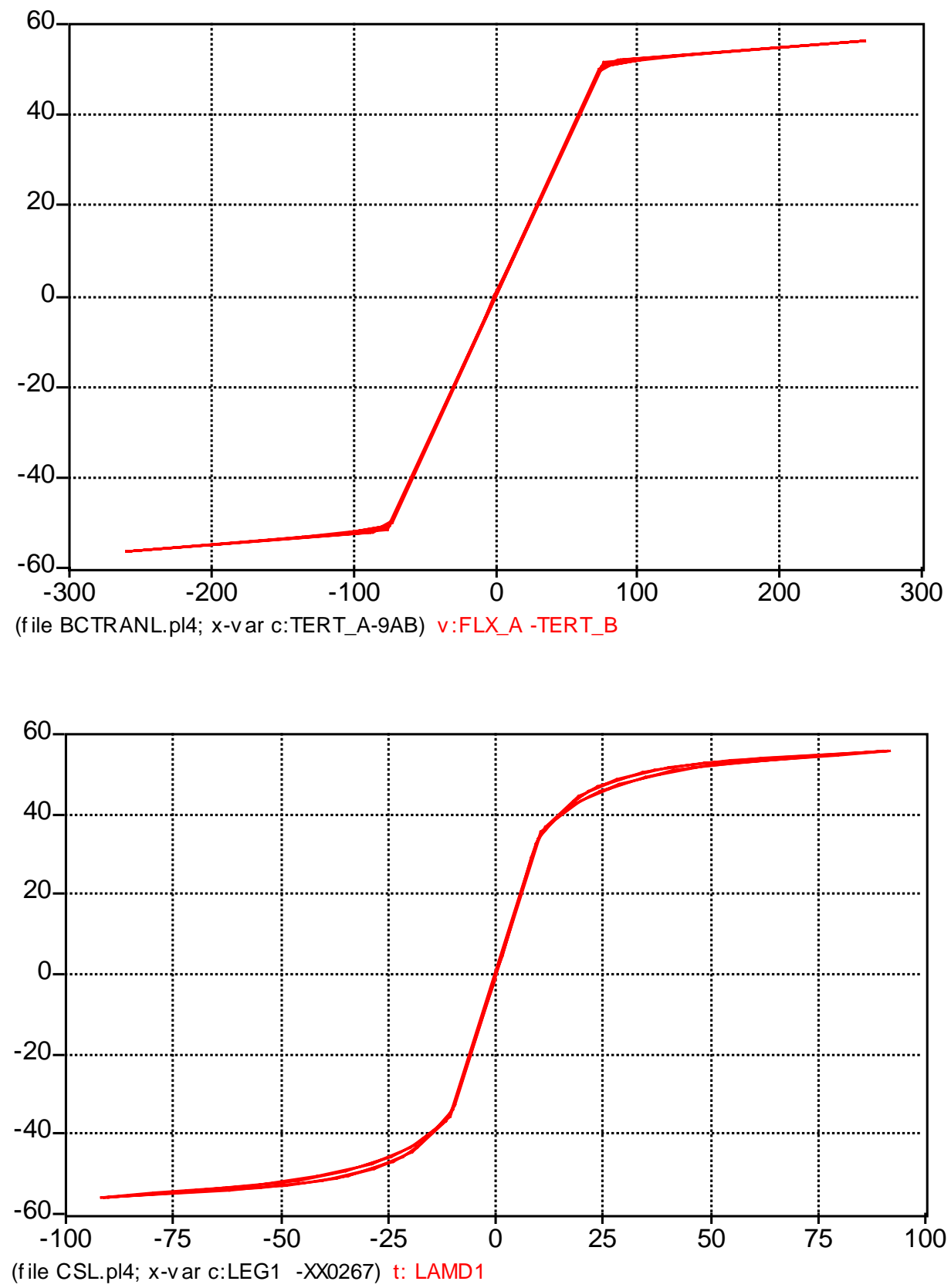

$\mathrm{X}$-axis: Current in Amperes on 13.8-kV base Y-axis: Flux Linkage in Wb-t BCTRAN Model (Top) and Duality Model (Bottom)

Figure 7.2 Transformer Core Flux - Magnetizing Current Plot at Leg-1 


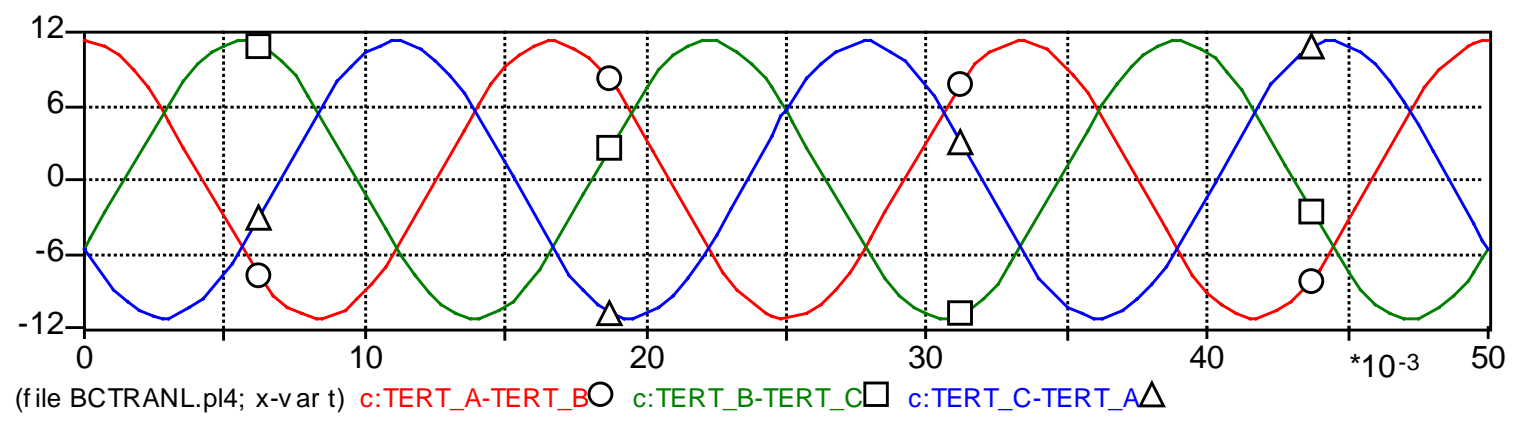

o: Phase-AB $\square$ : Phase-BC $\Delta$ : Phase-CA

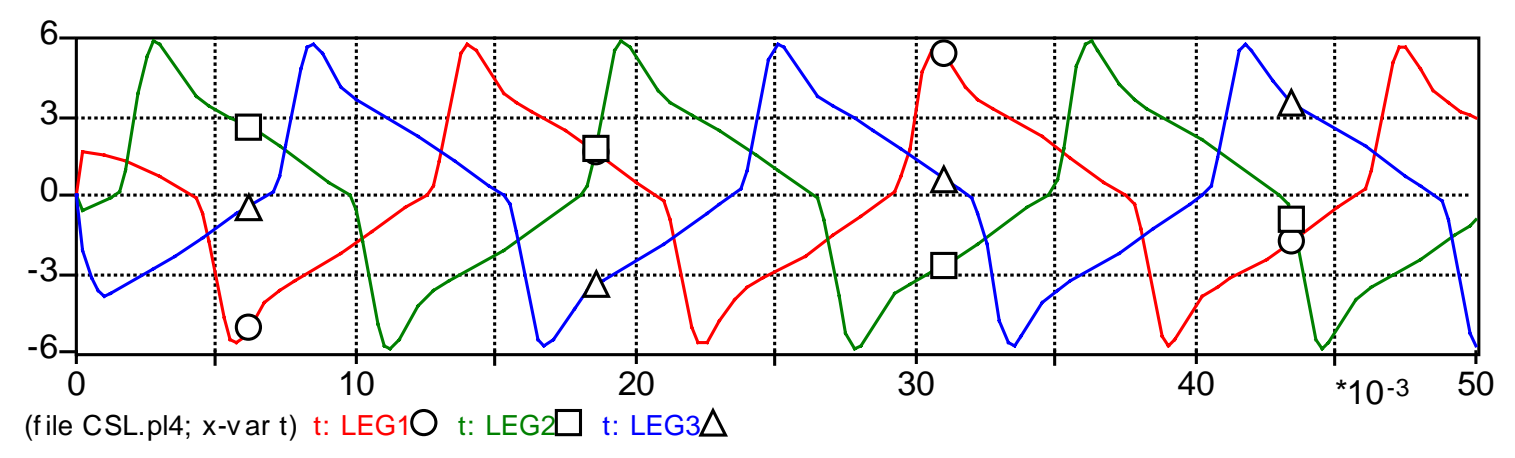

o: Leg-1 $\square$ : Leg-2 $\Delta$ : Leg-3

$\mathrm{X}$-axis: Time in Secs, Y-axis: Current in Amperes on $13.8-\mathrm{kV}$ base

BCTRAN Model (Top) and Duality Model (Bottom)

Figure 7.3 Transformer Core Loss Currents for Three Legs 

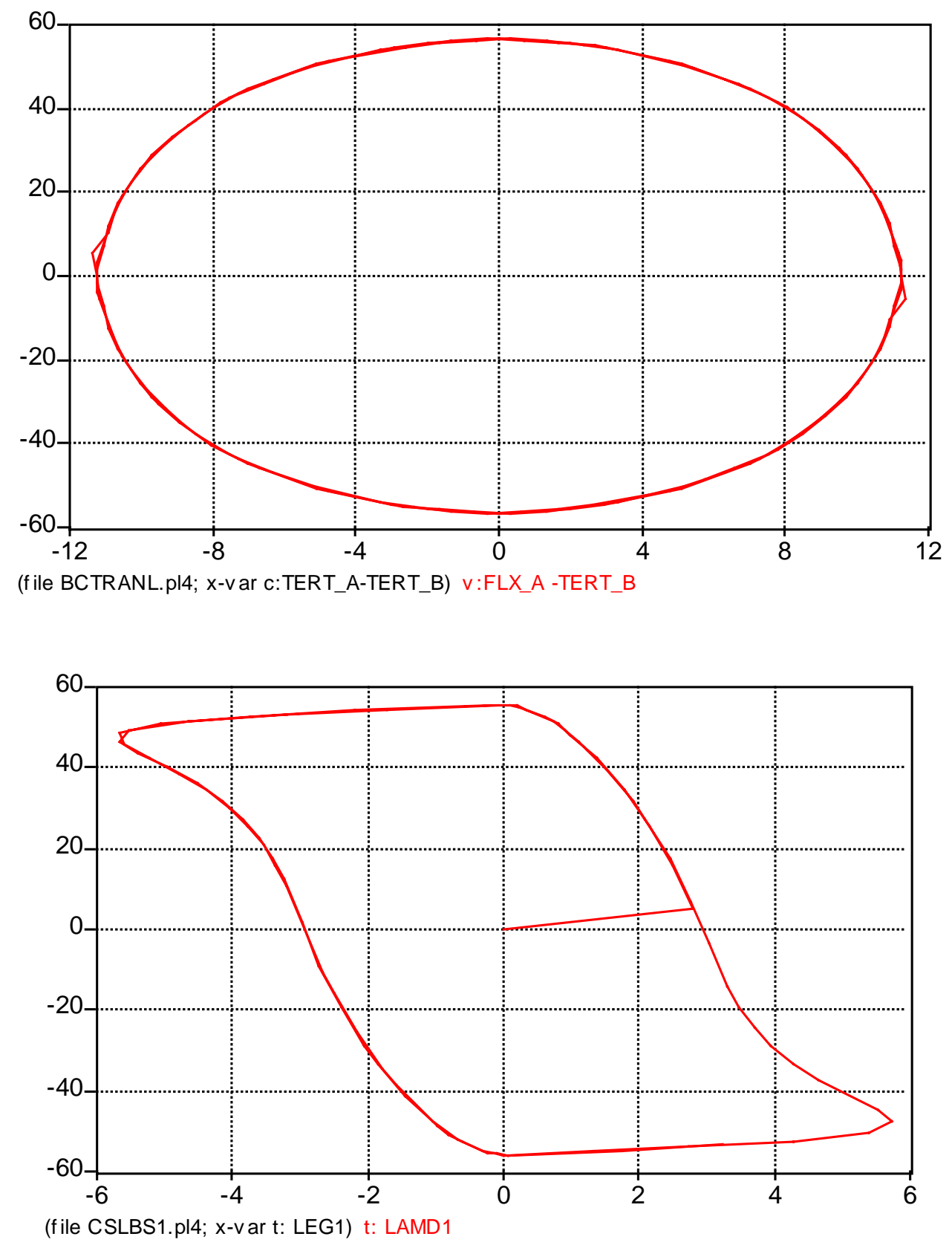

X-axis: Current in Amperes on 13.8-kV base Y-axis: Flux Linkage in Wb-t BCTRAN Model (Top) and Duality Model (Bottom)

Figure 7.4 Transformer Core Flux - Core Loss Current Plot for Leg-1 

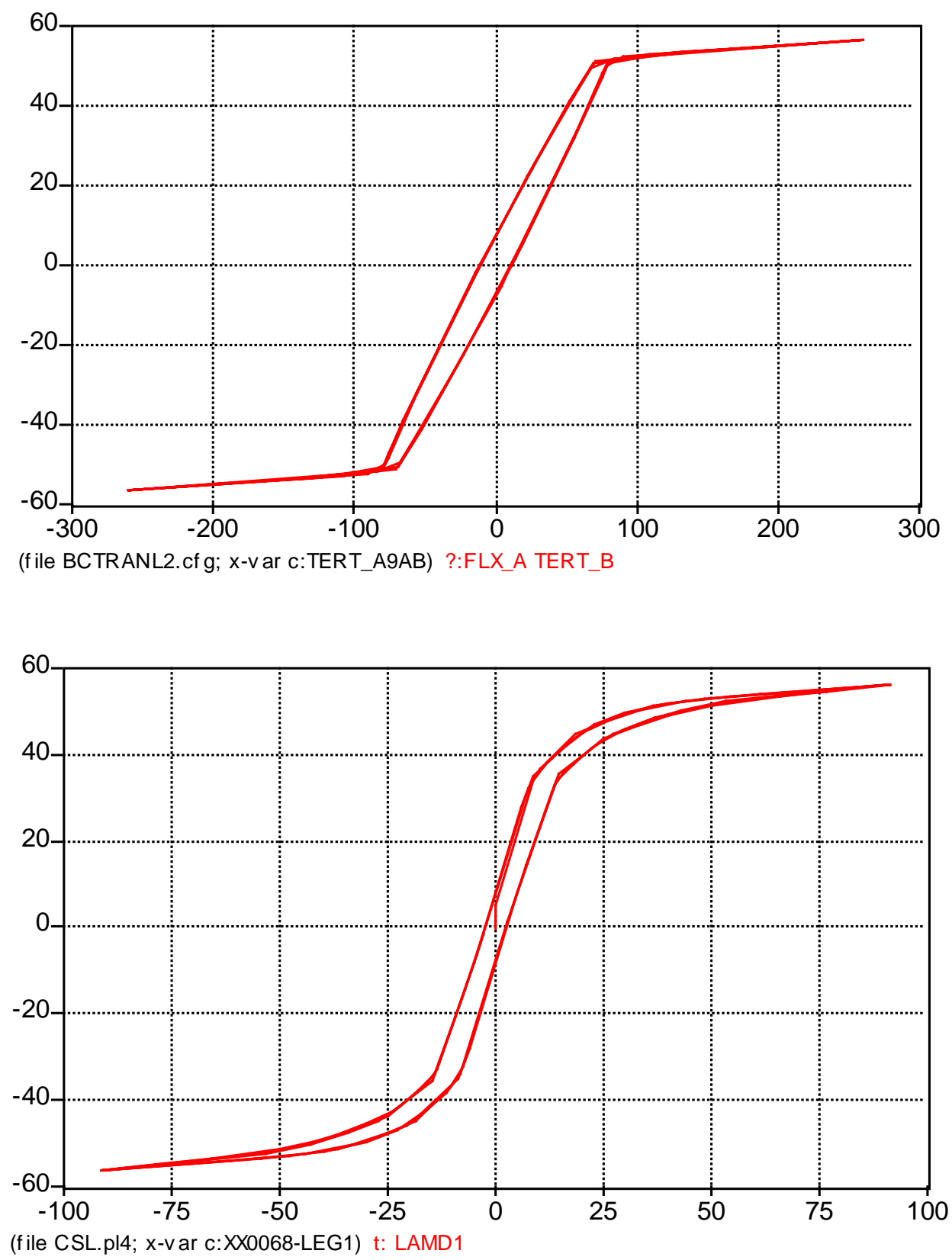

X-axis: Current in Amperes on 13.8-kV base Y-axis: Flux Linkage in Wb-t BCTRAN Model (Top) and Duality Model (Bottom)

Figure 7.5 Transformer Core Flux - No-Load Current Plot for Leg-1 

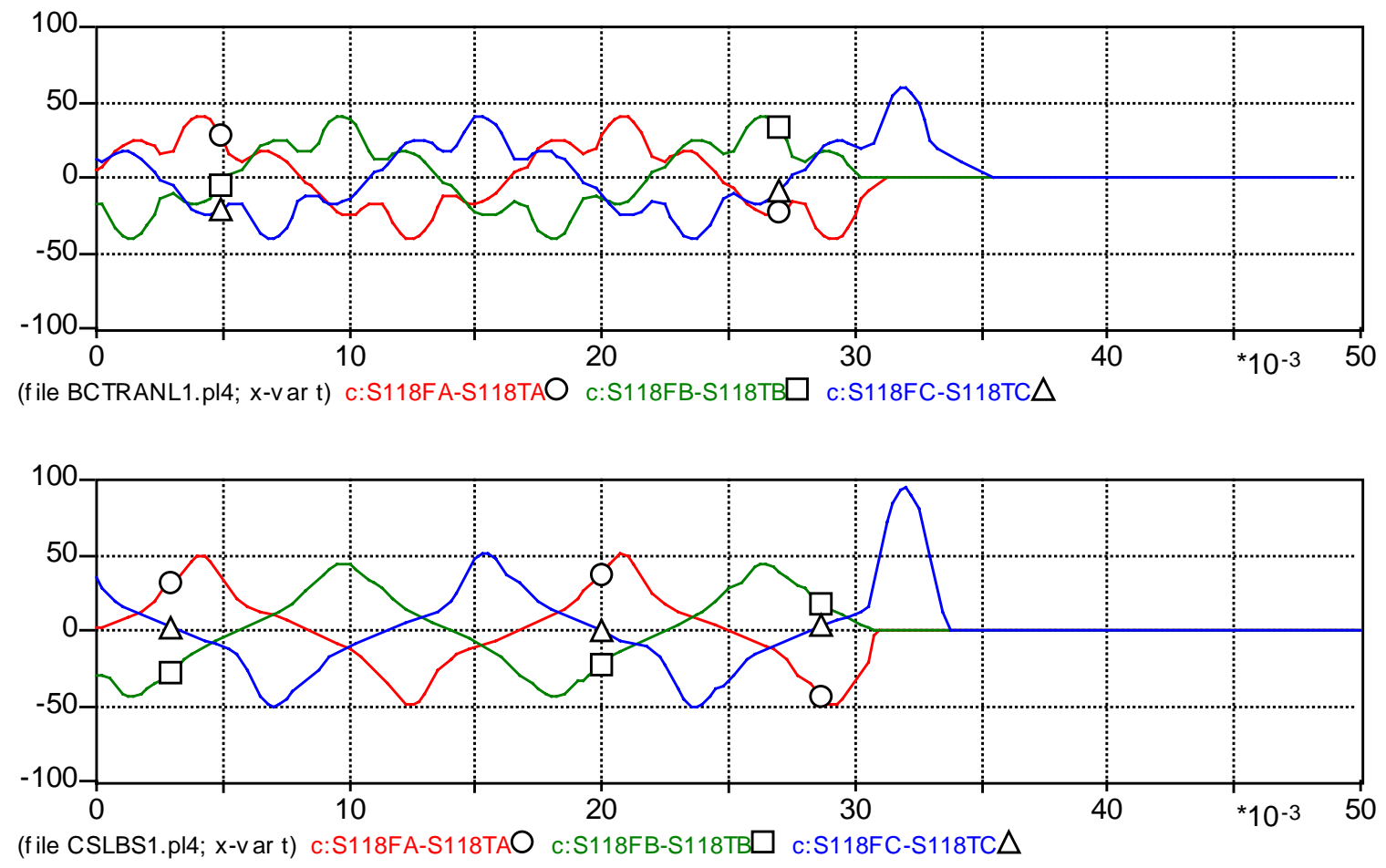

$\mathrm{X}$-axis: Time in Secs, Y-axis: Current in Amperes

o: Phase-A $\square$ : Phase-B $\Delta$ : Phase-C

BCTRAN Model (Top) and Duality Model (Bottom)

Figure 7.6 Transformer No-Load Currents for 115-kV Line Terminals 

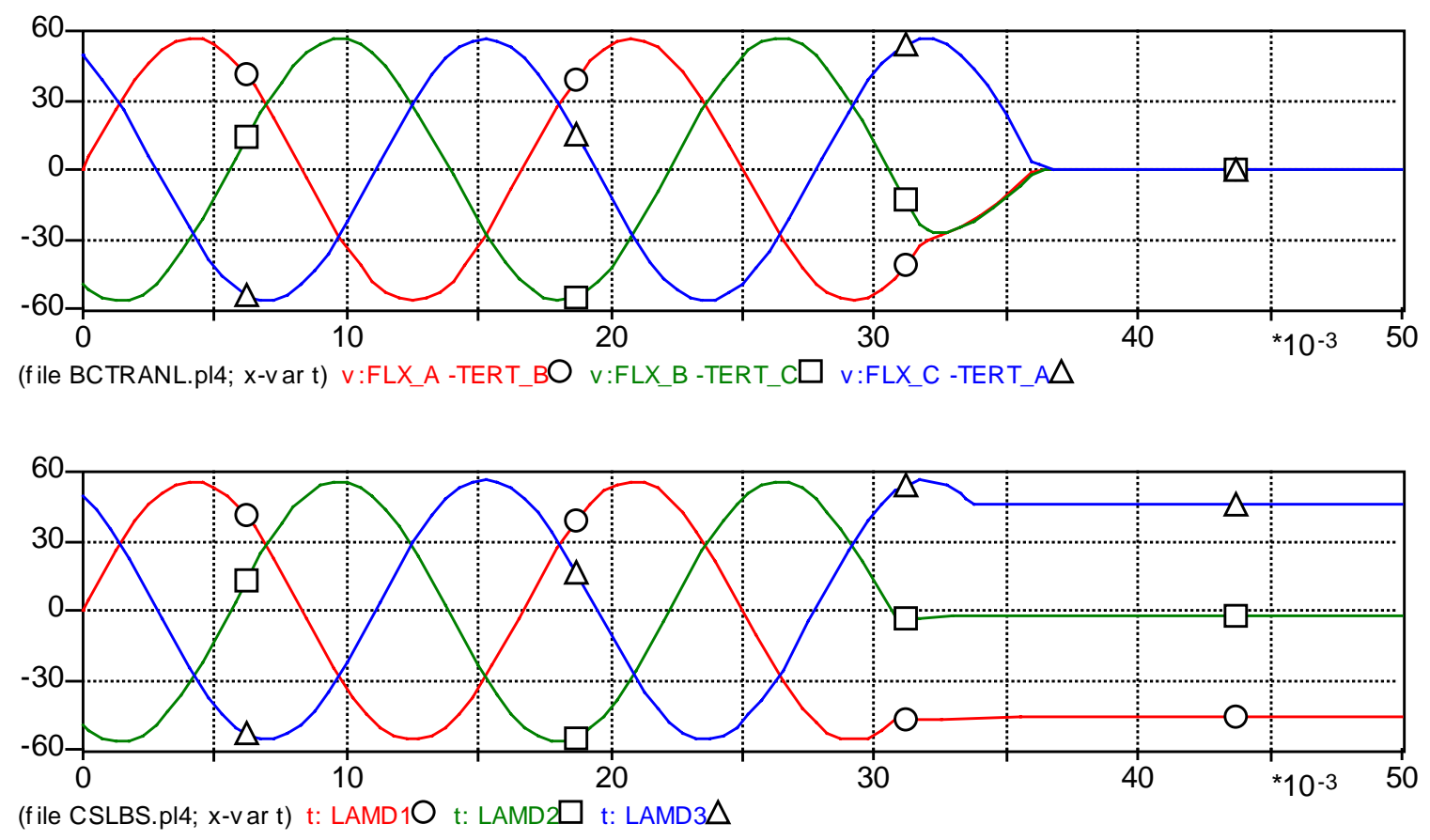

$\mathrm{X}$-axis: Time in Secs, Y-axis: Flux Linkage in Wb-t o: Leg-1 $\square$ : Leg-2 $\Delta$ : Leg-3

BCTRAN Model (Top) and Duality Model (Bottom)

Figure 7.7 Transformer Core Fluxes for Legs after De-Energizing 

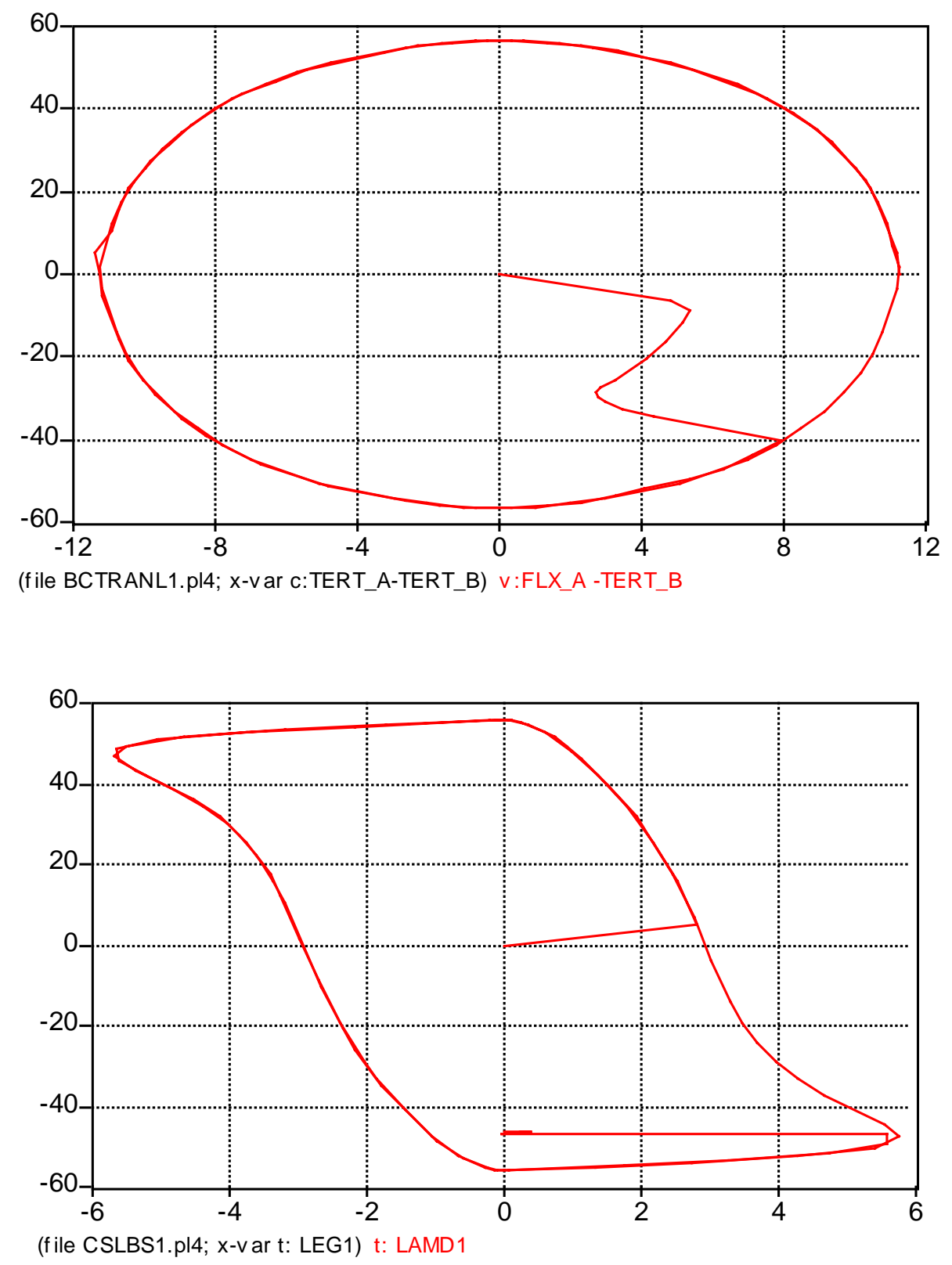

$\mathrm{X}$-axis: Current in Amperes on 13.8-kV base $\mathrm{Y}$-axis: Flux Linkage in Wb-t BCTRAN Model (Top) and Duality Model (Bottom)

Figure 7.8 Transformer Core Flux vs. Core Loss Current Plot After De-Energizing 

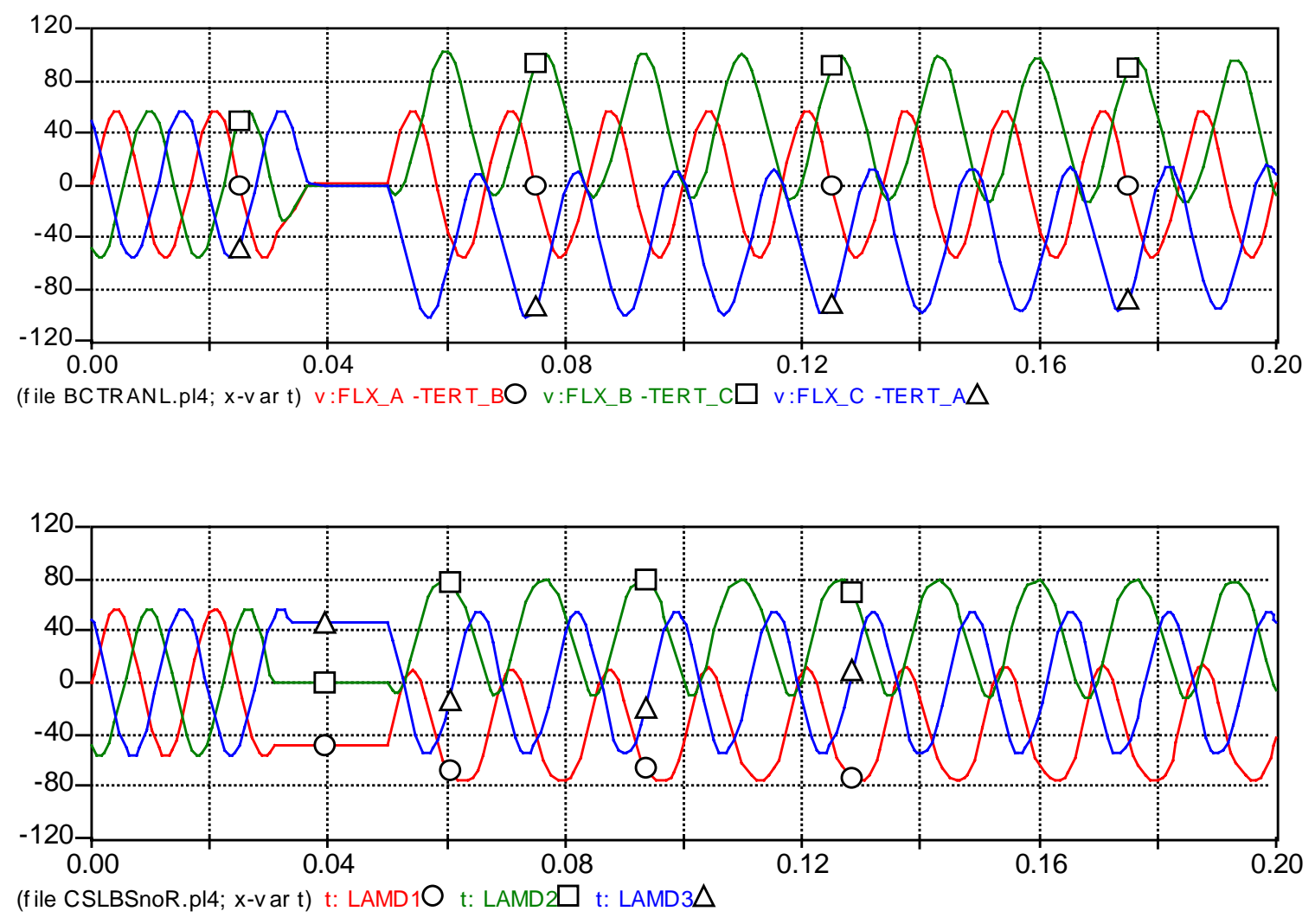

$\mathrm{X}$-axis: Time in Secs, Y-axis: Flux Linkage in Wb-t o: Leg-1 $\square$ : Leg-2 $\Delta$ : Leg-3

BCTRAN Model (Top) and Duality Model (Bottom)

Figure 7.9 Transformer Core Fluxes after Re-Energizing 

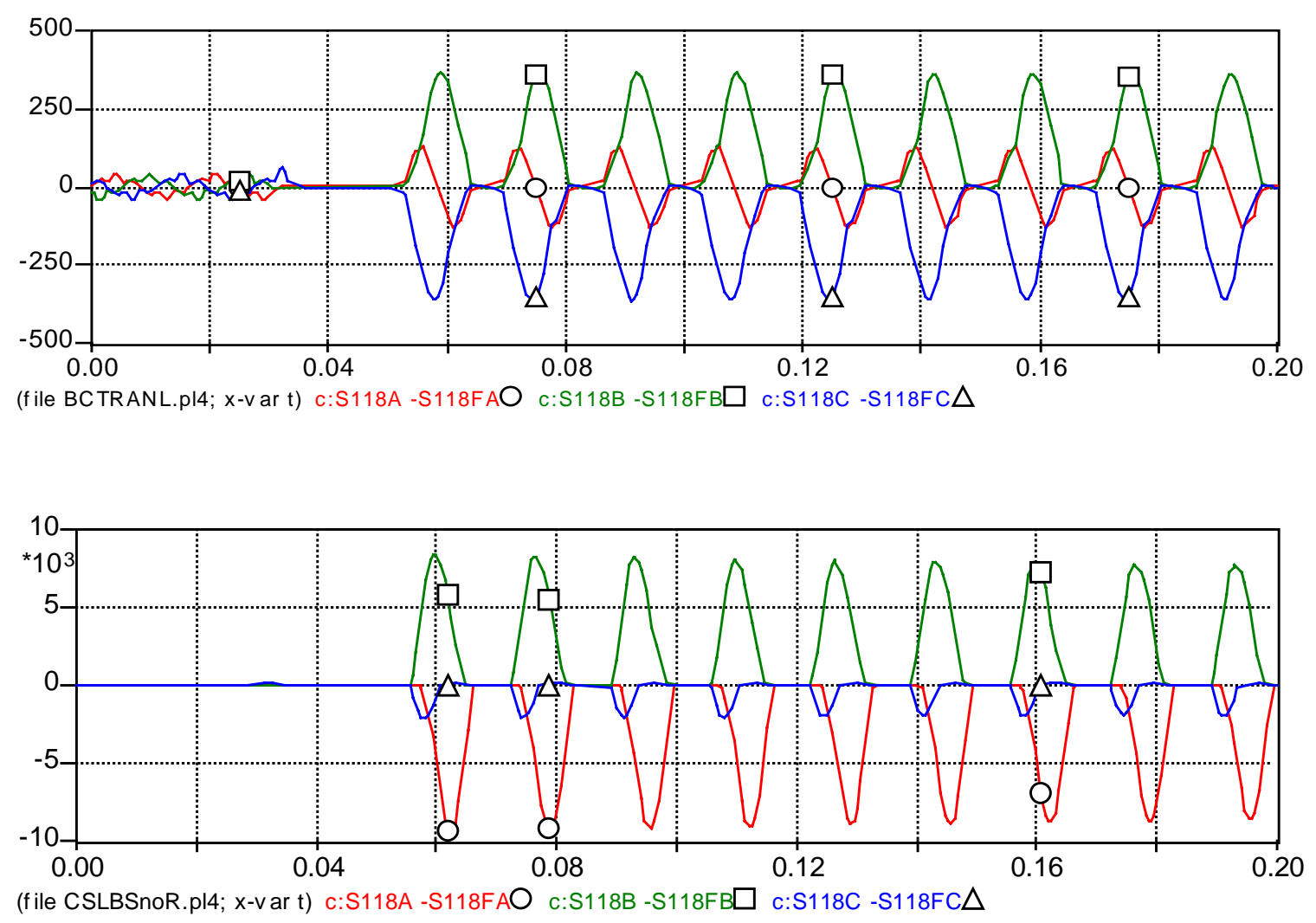

$\mathrm{X}$-axis: Time in Secs, Y-axis: Current in Amperes o: Phase-A $\square$ : Phase-B $\Delta$ : Phase-C BCTRAN Model (Top) and Duality Model (Bottom)

Figure 7.10 Transformer 115-kV Line Currents after Re-Energizing 


\subsection{Black Start Energization Cases at IVH Substation}

In this section, black start energization cases from a previous study [14] are simulated by ATP for model evaluation. The main event in a black start is the step-bystep energization of high voltage transmission lines through the low-voltage side of large

transformers. As such, transformers simultaneously experience low-side inrush and a through-current due to line energization on the high side. Since high inrush currents are possible, transformer core saturation can be a key aspect of the observed transient behaviors. Inrush currents are of relatively low frequency, but line energization currents can have high-frequency components. Transient overvoltages can also result, posing a threat to the equipment involved in the black start.

Black start test energization of a $345-\mathrm{kV}$ line and transformers from the gas turbine generators on the low-voltage side of the transformer was done during a black start test. This event, plus a general desire to be able to predict the transient voltage and current waveforms, resulted in the development of an ATP model. Three event records were available for benchmarking. They were taken at the substation that the transformer is located in. Comparisons of fault recorder waveforms with ATP simulation for two cases are provided here. The cases are:

1) $115-\mathrm{kV}$ CB 5P147 energization at IVH (Event Record: IVH55):

As initial conditions, gas turbine units 1 and 3 are running. IVH transformers Nos. 1 and 2 are energized in steady state. The IVH $115-\mathrm{kV}$ bus is energized and in steady state. The first event is triggered by closing the CB 5P147, which energizes the IVH transformer No.9 and the $345-\mathrm{kV}$ line to BLL.

2) Energization of 345-kV Transformer No.9 at BLL (Event Record: IVH57):

As initial conditions, the $345-\mathrm{kV}$ lines up to BLL and PKL are energized. 
The second event is triggered by closing the $\mathrm{CB}$, which energizes the BLL transformer No. 9.

\subsubsection{System Description}

The single-line diagram of Figure 7.11 provides a depiction of the power system and black start switching sequences. The model developed includes transformers Nos. 2, 3 and 9 at IVH, transformer No. 9 at BLL, the $345-\mathrm{kV}$ line from IVH to BLL, the $345-\mathrm{kV}$ line from BLL to PKL, and gas turbine generators Nos. 3 and 6.

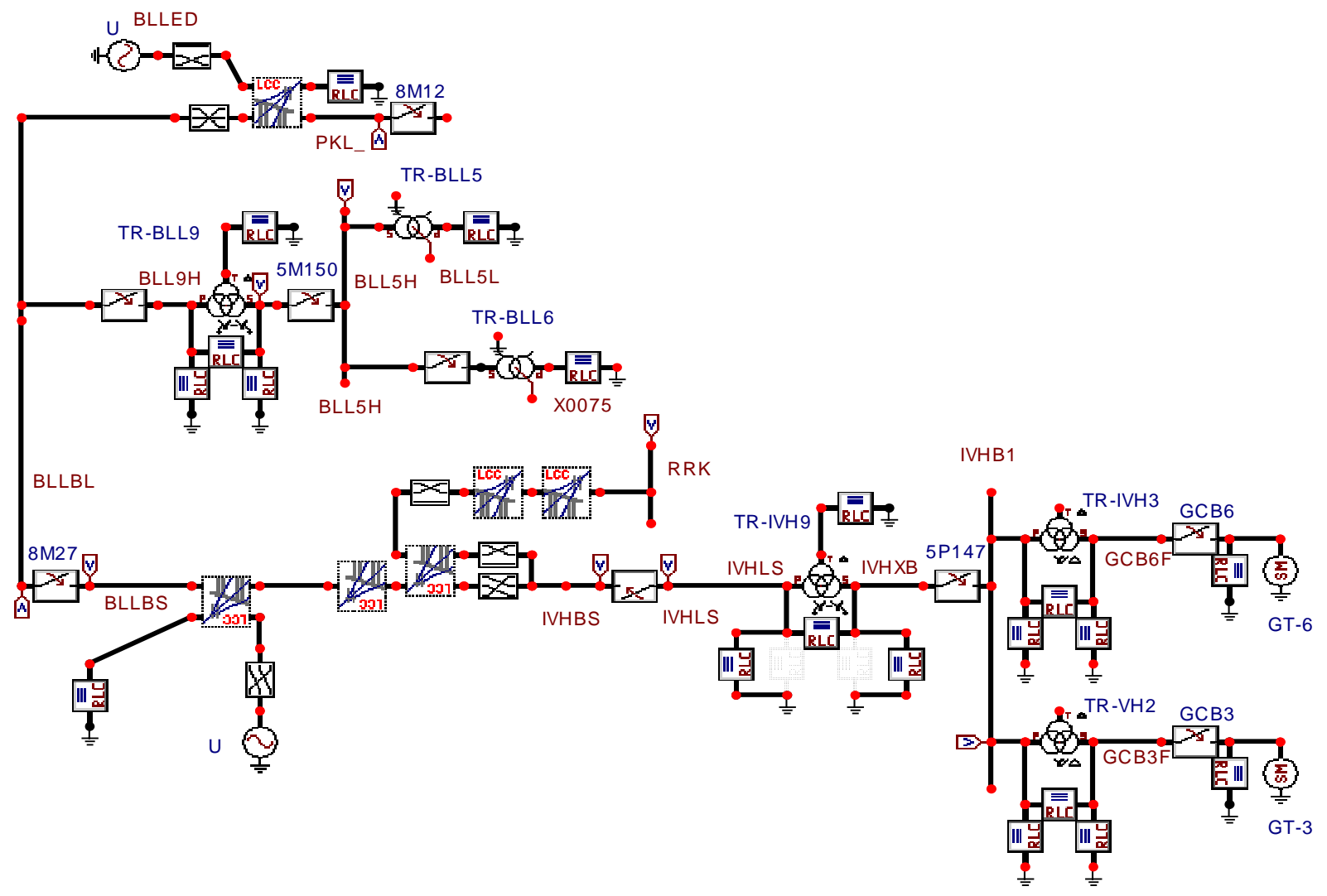

Figure 7.11 A Single Line Diagram for Black Start Study [14] 


\subsubsection{Transformer Model}

For the first simulation, IVH transformers 1, 2, and 9 and BLL transformer 9 are modeled using the BCTRAN-based model. The test data for the transformers are given in Tables 7.2 through 7.4. Core magnetization and losses are attached external to BCTRAN model, on the tertiary. Recall that the core is modeled with a type-98 inductance in parallel with a linear resistor. Using the $100 \%$ and $110 \%$ excitation data from the factory test report, the RMS magnetizing current is obtained by removing the core loss component from the exciting current. Three of these parallel R-L combinations are connected in delta and attached to the $13.8-\mathrm{kV}$ delta windings.

For the new simulation, all is the same as for the first simulation except IVH transformer 9 and BLL transformer 9 are modeled using the duality model for a shellform transformer. Core magnetization and losses are attached at the legs and yokes respectively. The core is modeled with a type-93 inductance in parallel with a type-60 TACS current source for hysteresis loss and eddy current loss. Although the factory test report gives $100 \%$ and $110 \%$ excitation data, more $\lambda$ - $i$ points are obtained from the core saturation function for the type-93 inductances.

Residual magnetism was not considered in this study. Since IVH transformer 9 is in place during line de-energization, it is expected that the line charging capacitance will "ring-down" together with the transformer, resulting in a near-zero residual magnetism. However, complete ringdown is usually not achieved, and a residual flux of as much as $30 \%$ of the peak steady-state flux might be expected. In the case of the BLL transformer No.9, a non-zero residual magnetism is expected, since it is separately de-energized. 
Table 7.2 Factory Test Data for Transformer No.9 at BLL

\begin{tabular}{|r|c|c|}
\hline \multicolumn{3}{|c|}{$\begin{array}{c}\text { 345000 Grd.Y/118000 Grd.Y/13800 Delta, } \\
\text { 3-phase auto-transformer @ OA/FOA/FOA } \\
\text { H- 180MVA, X-180MVA, Y-47.4MVA@OA }\end{array}$} \\
\cline { 2 - 3 } & Exciting Current & No Load Loss \\
\cline { 2 - 3 } & $0.87 \%$ @ 100\%Voltage & 191.48kW @ 100\%Voltage \\
\cline { 2 - 3 } Open-Circuit Test & $2.36 \%$ @ 110\%Voltage & 268.844kW @ 110\% Voltage \\
\hline Short-Circuit Test & Impedance & Load Loss \\
\hline H-X & $6.77 \% @ 180 \mathrm{MVA}$ & 275.871kW @ 180MVA \\
\hline $\mathrm{H}-\mathrm{Y}$ & $51.7 \%, @ 180 \mathrm{MVA}$ & $75.997 \mathrm{~kW} @ 47.4 \mathrm{MVA}$ \\
\hline $\mathrm{X}-\mathrm{Y}$ & $37.3 \%$ @ 180MVA & $78.856 \mathrm{~kW} @ 47.4 \mathrm{MVA}$ \\
\hline
\end{tabular}

Table 7.3 Factory Test Data for Transformers 1 at IVH

\begin{tabular}{|r|c|c|}
\hline \multicolumn{3}{|c|}{$125 / 62.5 / 62.5 \mathrm{MVA} 124 / 14.4 / 14.4 \mathrm{kV}$ Y-D-D } \\
\hline \multirow{2}{*}{ Open-Circuit Test } & Exciting Current & No Load Loss \\
\cline { 2 - 3 } & $0.21 \%$ @ 100\% Voltage & $92-\mathrm{kW} @ 100 \%$ Voltage \\
\cline { 2 - 3 } & $0.52 \%$ @ 110\% Voltage & $131.84-\mathrm{kW} @ 110 \%$ Voltage \\
\hline Short-Circuit Test & Impedance & Load Loss \\
\hline $\mathrm{H}-\mathrm{X}$ & $9.9 \%$ @ 125-MVA & $227.2-\mathrm{kW} @ 62.5-\mathrm{MVA}$ \\
\hline $\mathrm{H}-\mathrm{Y}$ & $9.99 \%$ @ $125-\mathrm{MVA}$ & $231.4-\mathrm{kW} @ 62.5-\mathrm{MVA}$ \\
\hline $\mathrm{X}-\mathrm{Y}$ & $18.61 \% @ 125-\mathrm{MVA}$ & $419.8-\mathrm{kW} @ 62.5-\mathrm{MVA}$ \\
\hline
\end{tabular}

Table 7.4 Factory Test Data for Transformers 2 at IVH

\begin{tabular}{|r|c|c|}
\hline \multirow{3}{*}{$125 / 62.5 / 62.5 \mathrm{MVA} 124 / 14.4 / 14.4 \mathrm{kV}$ Y-D-D } \\
\hline \multirow{2}{*}{ Open-Circuit Test } & Exciting Current & No Load Loss \\
\cline { 2 - 3 } & $0.29 \%$ @ 100\%Voltage & 97.9-kW @ 100\%Voltage \\
\cline { 2 - 3 } & $0.65 \%$ @ 110\%Voltage & $134.4-\mathrm{kW} @ 110 \%$ Voltage \\
\hline Short-Circuit Test & Impedance & Load Loss \\
\hline $\mathrm{H}-\mathrm{X}$ & $9.87 \%$ @ 125-MVA & $219.7-\mathrm{kW} @ 62.5-\mathrm{MVA}$ \\
\hline $\mathrm{H}-\mathrm{Y}$ & $10.0 \%$ @ 125-MVA & $222.6-\mathrm{kW} @ 62.5-\mathrm{MVA}$ \\
\hline $\mathrm{X}-\mathrm{Y}$ & $18.28 \%$ @ 125-MVA & $395.0-\mathrm{kW} @ 62.5-\mathrm{MVA}$ \\
\hline
\end{tabular}

\subsubsection{Transmission Line Models}

The line sections for the IVH to BLL line and the BLL to PKL line are modeled with JMARTI in ATP. The IVH to BLL line is mutually coupled with a parallel line for 
18.23 miles of its 22.59 miles. The BLL to PKL line is mutually coupled with a parallel line for all of its 14.86 miles. Verification for input data involves positive and zero sequence impedances, zero sequence coupling, and line charging MVAR.

\subsubsection{Synchronous Generator Model}

These generators are represented as a detailed synchronous generator. The IEEE Type 3 excitation system shown in Figure 7.12 was used to represent the exciter/voltage regulator dynamics [14]. Note that the excitation system has a very large time constant. Regulator time constant $\mathrm{T}_{\mathrm{A}}$ is $0.15 \mathrm{~s}$ and the exciter time constant $\mathrm{T}_{\mathrm{E}}$ is $0.5 \mathrm{~s}$. Governor models were not added. Maximum generator reactive capability is about 27 MVar at $100 \%$ leading power factor. Maximum generator reactive capability limits were not added to the model.

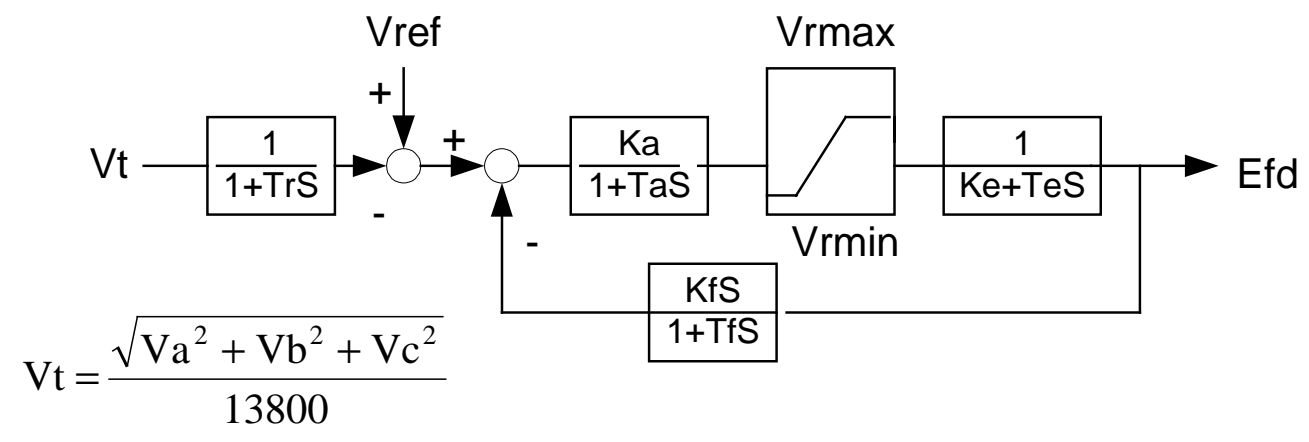

IEEE Type 3 excitation system

Regulator time constant Ta 0.15s, Gain Ka 120, Regulator Input Filter Time constant Tr 0s, Exciter time constant Te $0.5 \mathrm{~s}$, Constant related to self-excited field Ke 1.0, Regulator Stabilizing Circuit time constant Tf $0.461 \mathrm{~s}$, Gain Kf 0.2 , Vrmax 1.2, Vrmin -1.2

Two Gas Turbine Generators:

55 MVA, $13.8 \mathrm{kV} X " d v=0.138 \mathrm{pu}, \mathrm{X}$ 'di=0.138pu, $\mathrm{R}_{1}=0.003 \mathrm{pu}, \mathrm{X}_{0}=0.059, \mathrm{X}_{2}=0.097 \mathrm{pu}$

Figure 7.12 Block Diagram for Generator Excitation System 


\subsubsection{Case Study Results}

The voltages and currents at IVH are shown in the attached plots. To simulate initial conditions similar to the fault recorder, the following switching sequences were assumed:

Fault Recorder Case: IVH55

\begin{tabular}{|c|l|}
\hline Time (sec) & \multicolumn{1}{c|}{ Case Description } \\
\hline 0 & $\begin{array}{l}\text { At IVH, with both generators running, both 13.8/115-kV generator } \\
\text { step-up transformers are energized. }\end{array}$ \\
\hline 0.096 & 115-kV CB 5P147 energization at IVH \\
\hline
\end{tabular}

Fault Recorder Case: IVH57

\begin{tabular}{|c|l|}
\hline Time (sec) & \multicolumn{1}{|c|}{ Case Description } \\
\hline 0 & $\begin{array}{l}\text { At IVH, with both generators running, both 13.8/115-kV generator } \\
\text { step-up transformers and 115-kV CB 5P147 are energized. 345-kV } \\
\text { CB 8M27 at BLL are energized. }\end{array}$ \\
\hline 0.099 & Energization of 345-kVTransformer No.9 at BLL \\
\hline
\end{tabular}

Figures 7.13 through 7.18 compare the fault recorder waveforms to ATP simulations for case IVH55 and IVH57. The behaviors of simulation using duality model match better with the event record than those of simulation using the previous BCTRAN model.

The low-frequency oscillations in black start are strongly related to transformer core saturation effects. Note that The $100 \%$ and $110 \%$ factory excitation data were used for the model parameters. However, the $110 \%$ excitation level was being exceeded in many cases. 
In the case of the BCTRAN model, the $100 \%$ and $110 \%$ excitation data from the factory test report were used for the nonlinear inductances. However, in case of the duality model, fourteen $\lambda-i$ points from the core saturation function were used as piecewise linear (See Appendices A.2 A.11) and the results obtained are more accurate.

In the case of $115-\mathrm{kV}$ CB 5P147 energization at IVH, the voltages of the dualitybased model simulation better match those of the fault recorder. Before and after the line energization, the line-charging currents of the ATP simulations are larger than those of the fault recorder. This might be caused by inaccurate line configuration data.

In the case of energization of $345-\mathrm{kV}$ transformer No.9 at BLL, the voltages of the duality model simulations better match those of the fault recorder. The currents (the inrush currents) of the ATP simulations have less 5th harmonic component than those of fault recorder.

The discrepancies of the current waveforms are caused by different initial conditions given at the beginning of the simulations. Initial conditions could not be accurately estimated because of the lack of line configuration data. 


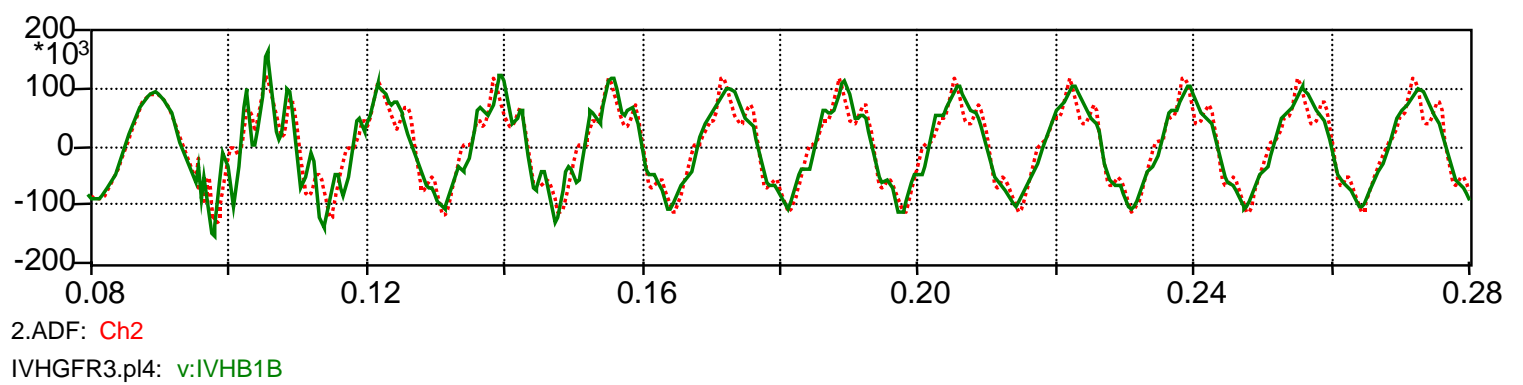

dotted line: Fault Recorder solid line: BCTRAN model

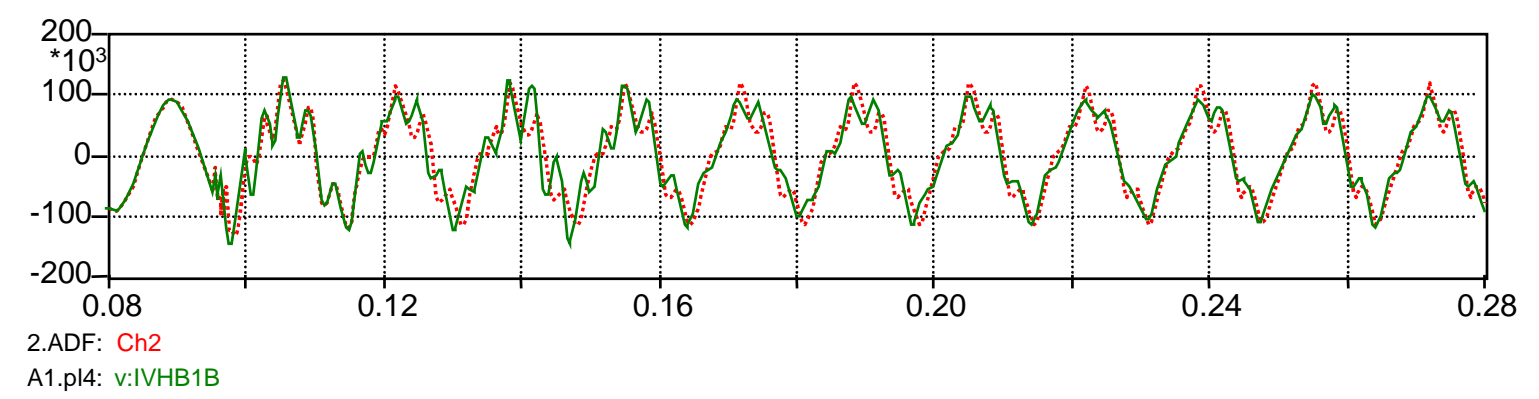

dotted line: Fault Recorder solid line: Duality model

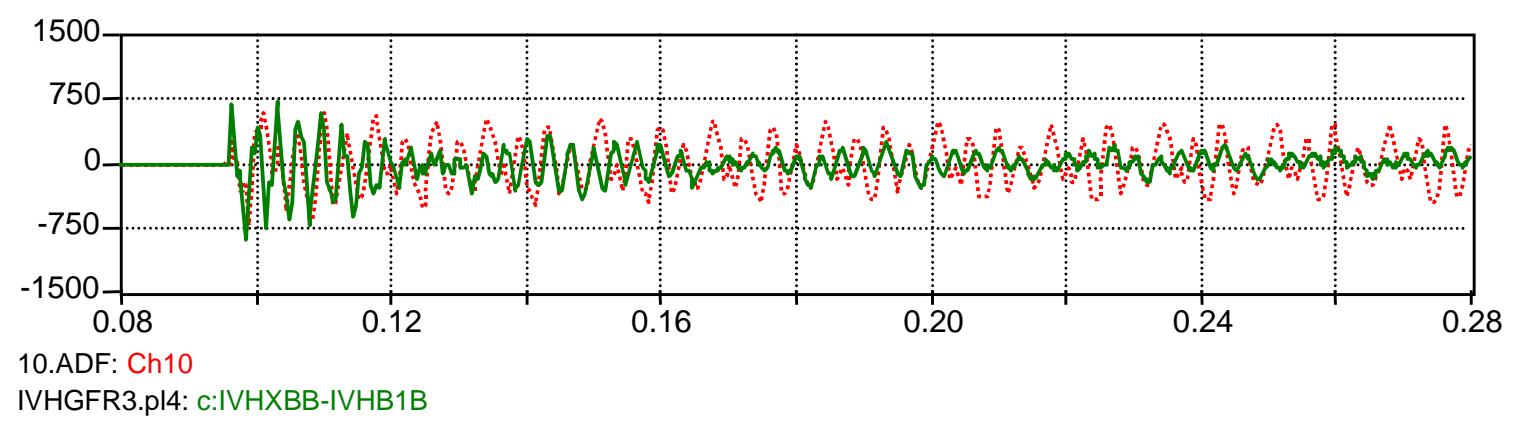

dotted line: Fault Recorder solid line: BCTRAN model

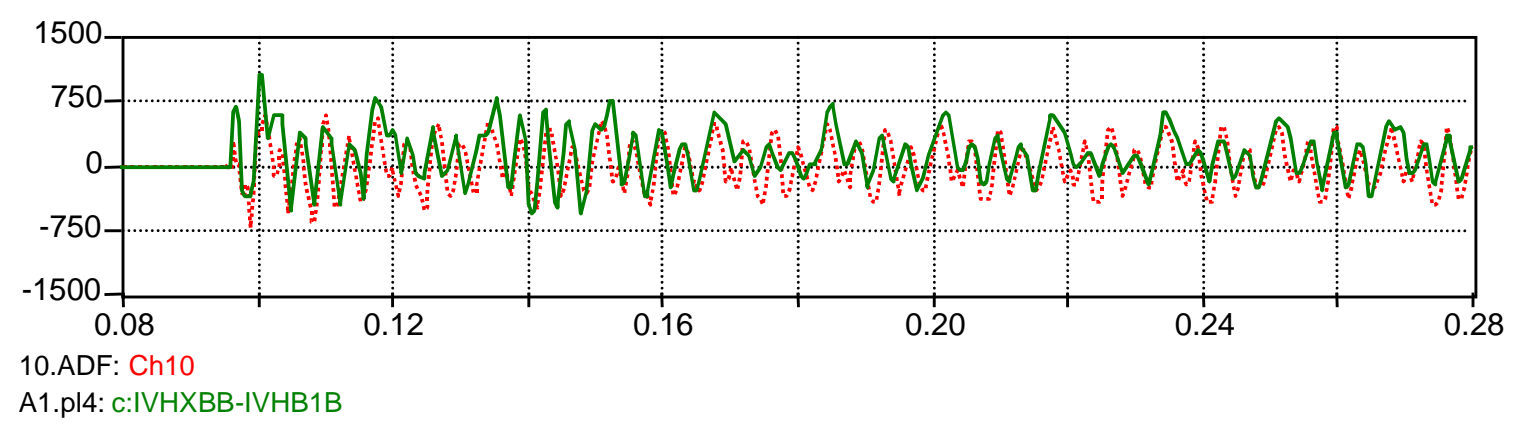

dotted line: Fault Recorder solid line: Duality model

X-axis: Time in Secs, Y-axis: Voltage in Volt (Top), Current in Amperes (Bottom)

Figure 7.13 115-kV CB 5P147 B-phaseVoltage (Top) and Current (Bottom) Just after 115-kV CB 5P147 Energization 


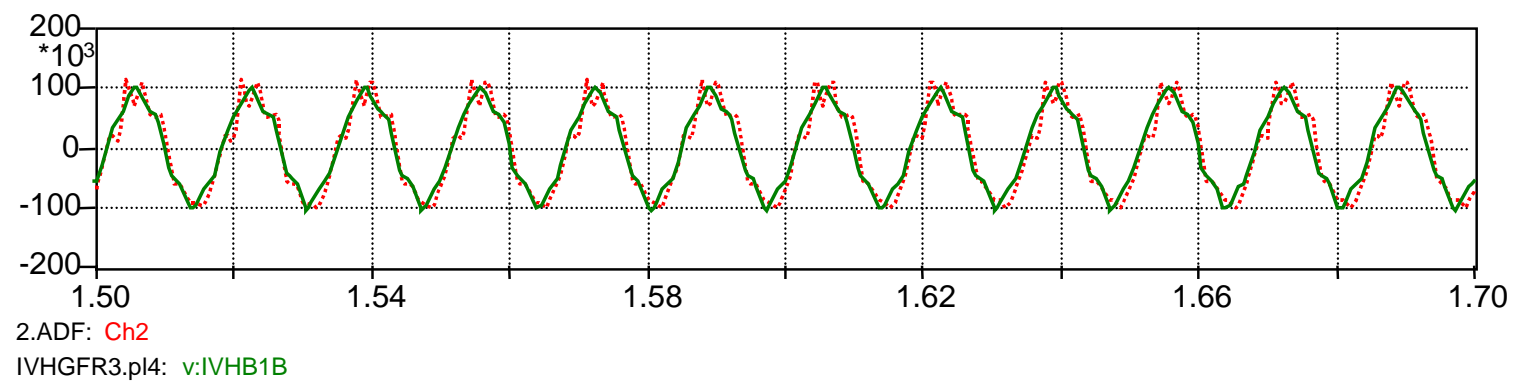

dotted line: Fault Recorder solid line: BCTRAN model

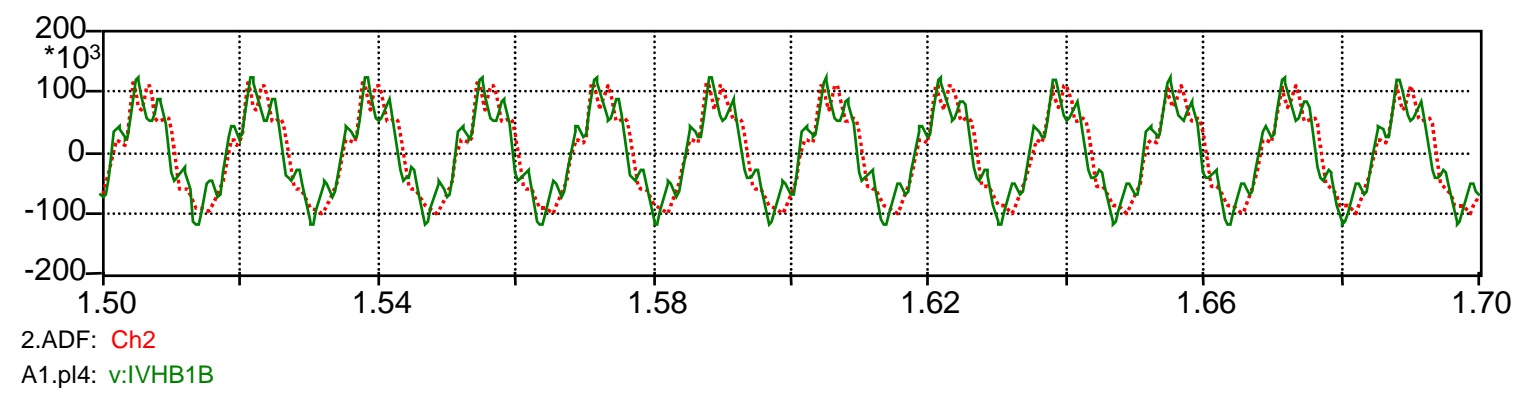

dotted line: Fault Recorder solid line: Duality model

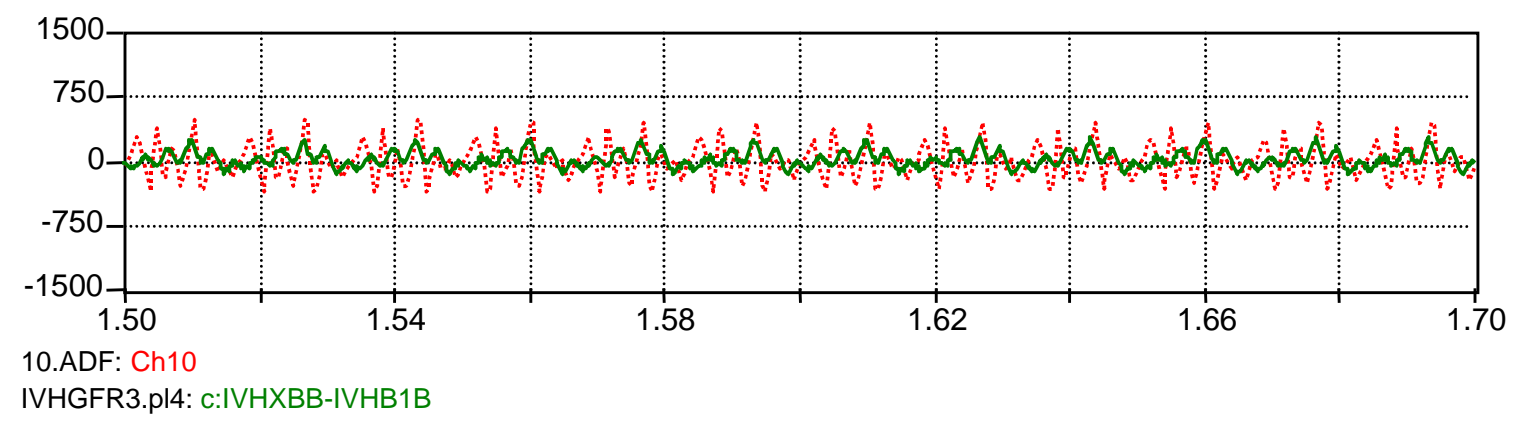

dotted line: Fault Recorder solid line: BCTRAN model

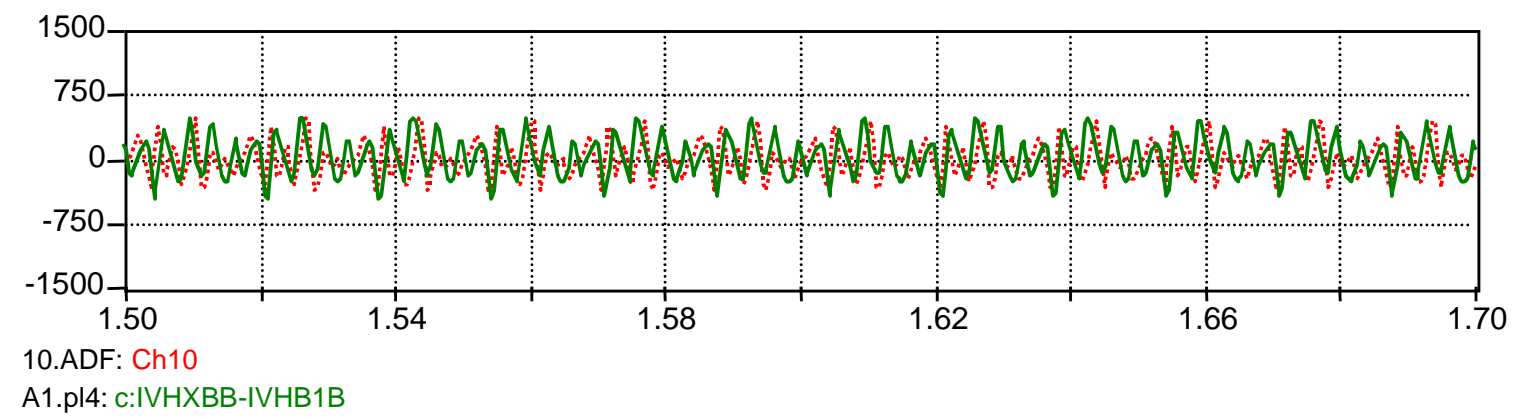

dotted line: Fault Recorder solid line: Duality model

X-axis: Time in Secs, Y-axis: Voltage in Volt (Top), Current in Amperes (Bottom)

Figure 7.14 115-kV CB 5P147 B-phase Voltage (Top) and Current (Bottom) 1.5 seconds after 115-kV CB 5P147 Energization 


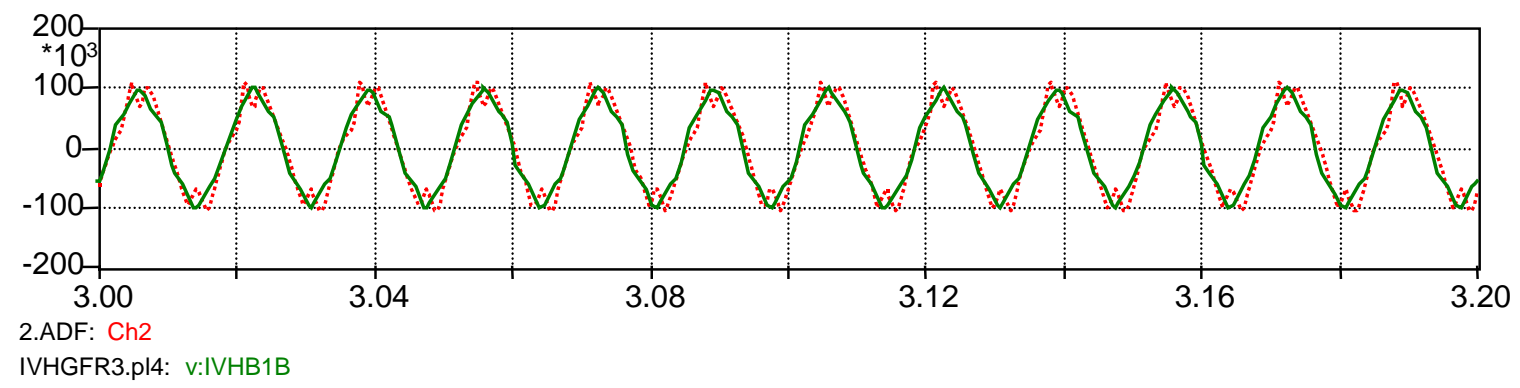

dotted line: Fault Recorder solid line: BCTRAN model

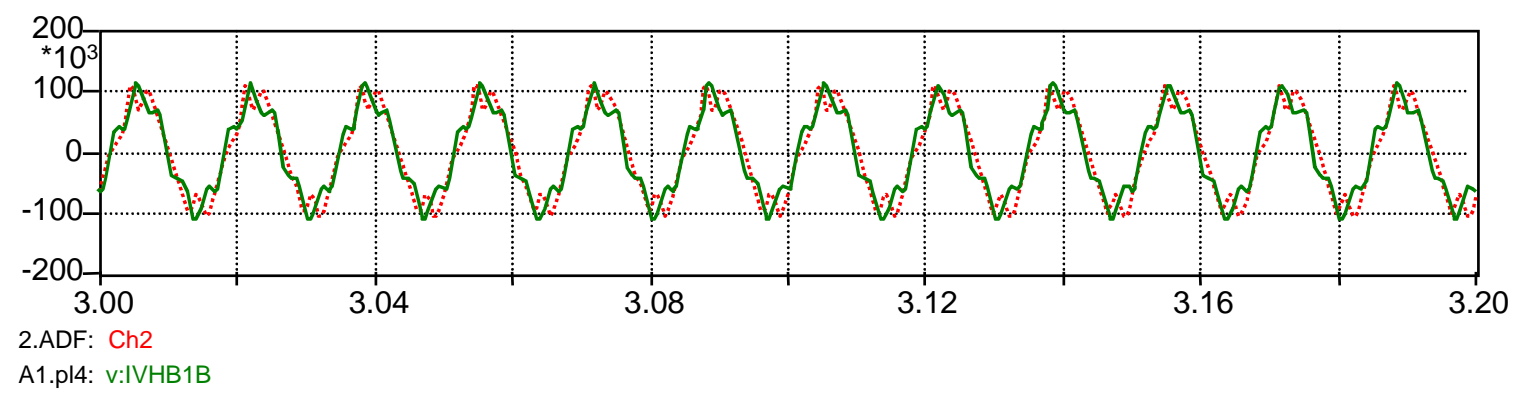

dotted line: Fault Recorder solid line: Duality model

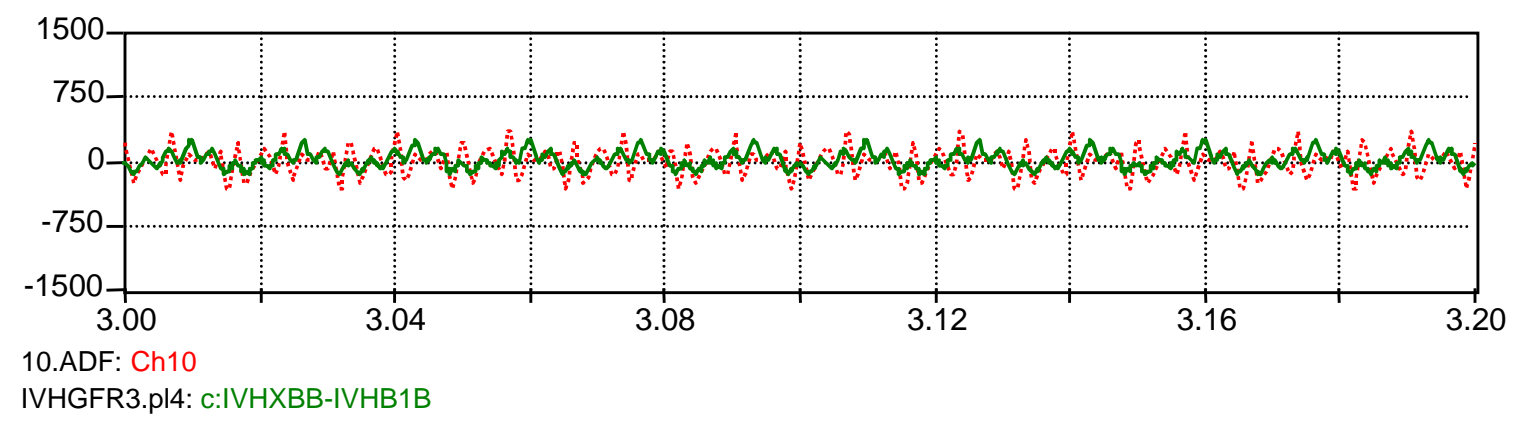

dotted line: Fault Recorder solid line: BCTRAN model

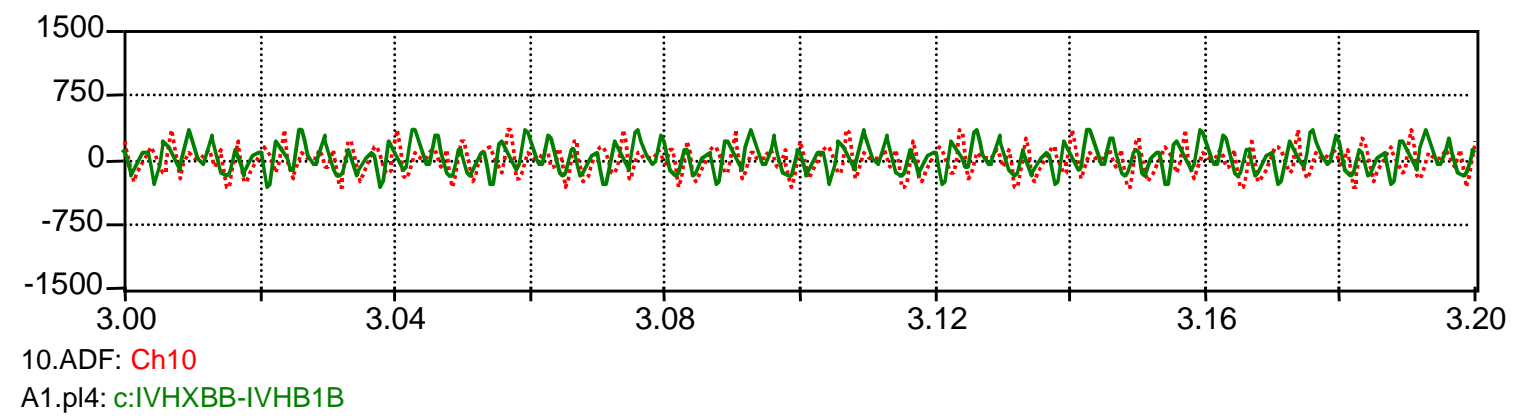

dotted line: Fault Recorder solid line: Duality model

X-axis: Time in Secs, Y-axis: Voltage in Volt (Top), Current in Amperes (Bottom)

Figure 7.15 115-kV CB 5P147 B-phase Voltage (Top) and Current (Bottom) 3 seconds after 115-kV CB 5P147 Energization 


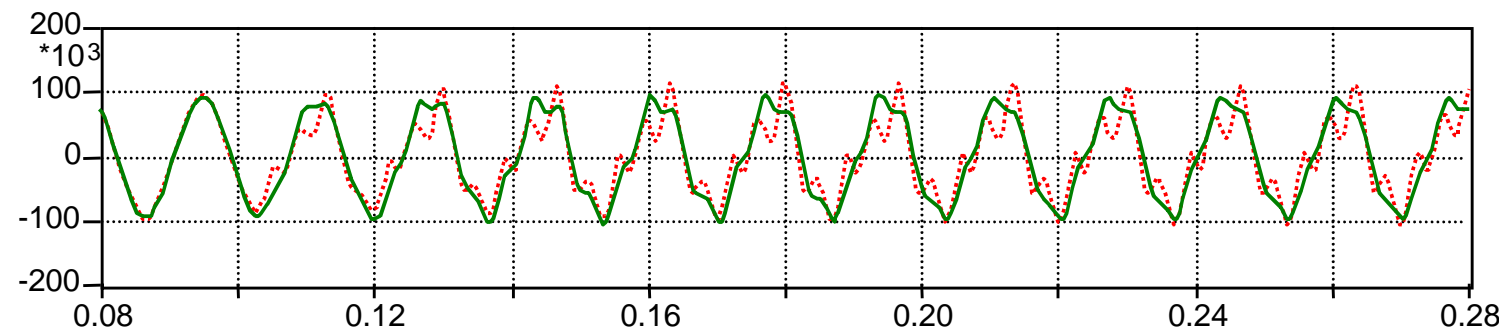

A3.ADF: Ch3

IVHGFR3B.pl4: v:IVHB1C

dotted line: Fault Recorder solid line: BCTRAN model

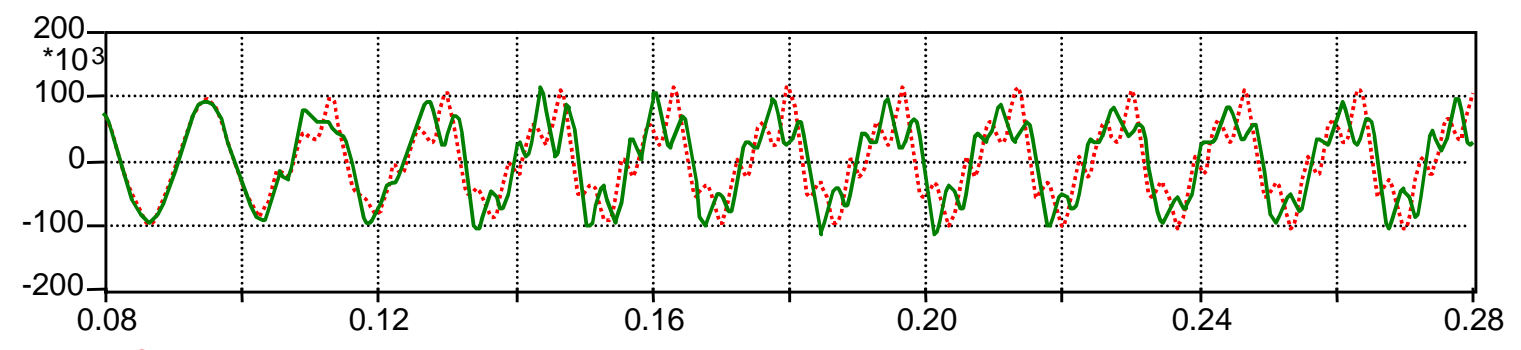

A3.ADF: Ch3

B1.pl4:v:IVHB1C

dotted line: Fault Recorder solid line: Duality model

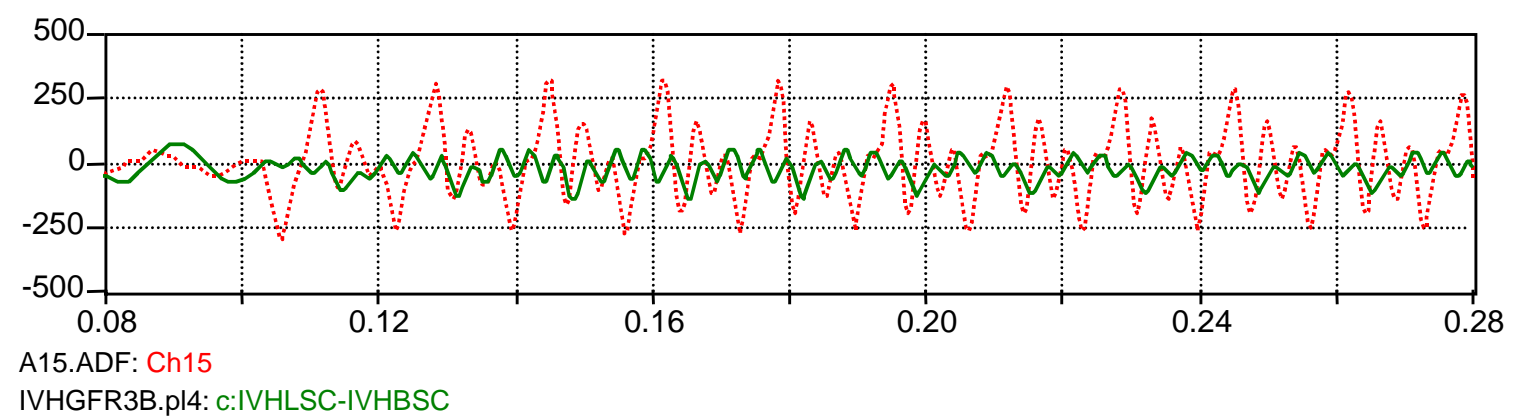

dotted line: Fault Recorder solid line: BCTRAN model

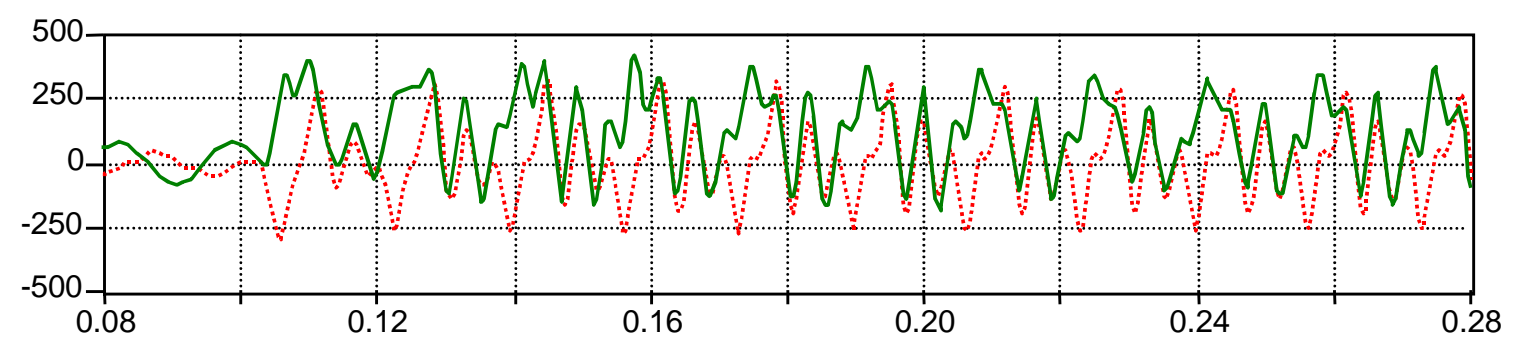

A15.ADF: Ch15

B1.pl4: c:IVHLSC-IVHBSC

dotted line: Fault Recorder solid line: Duality model

X-axis: Time in Secs, Y-axis: Voltage in Volt (Top), Current in Amperes (Bottom)

Figure 7.16 115-kV CB 5P147 C-phase Voltage (Top) and Current (Bottom) Just after Energization of 345-kV Transformer No.9 at BLL 


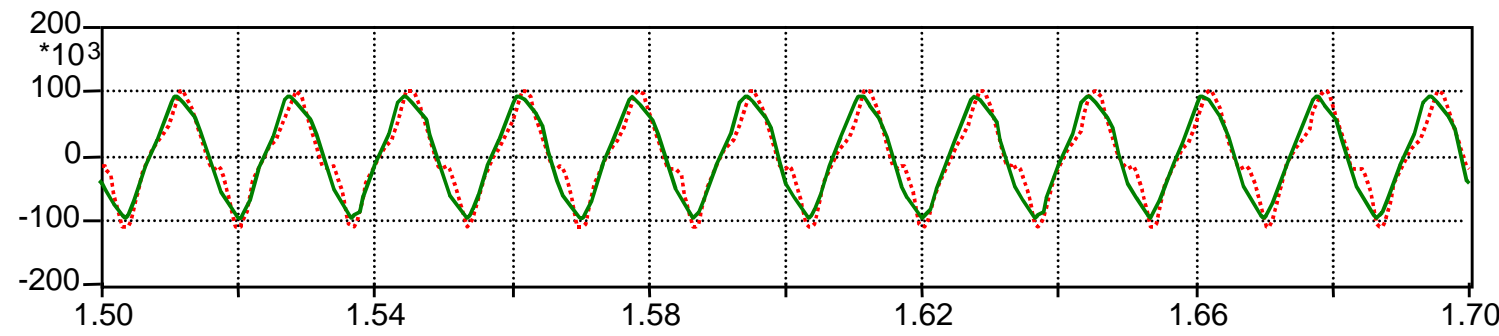

A3.ADF: Ch3

IVHGFR3B.pl4: v:IVHB1C

dotted line: Fault Recorder solid line: BCTRAN model

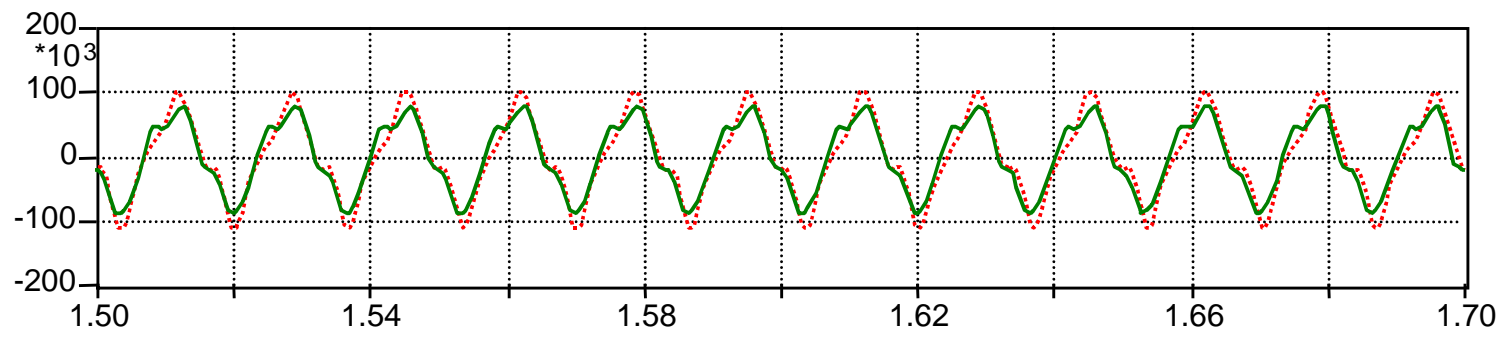

A3.ADF: Ch3

B1.pl4: v:IVHB1C

dotted line: Fault Recorder solid line: Duality model

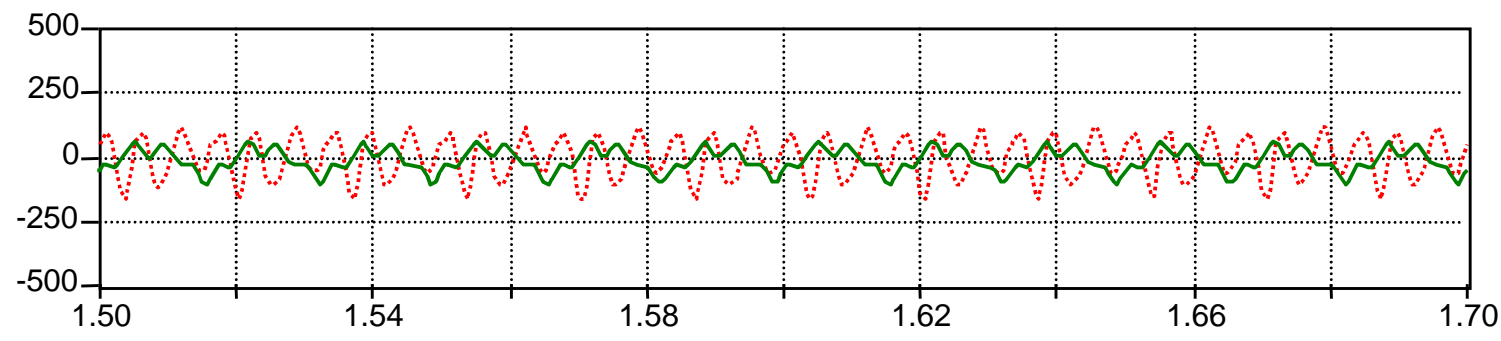

A15.ADF: Ch15

IVHGFR3B.pl4: c:IVHLSC-IVHBSC

dotted line: Fault Recorder solid line: BCTRAN model

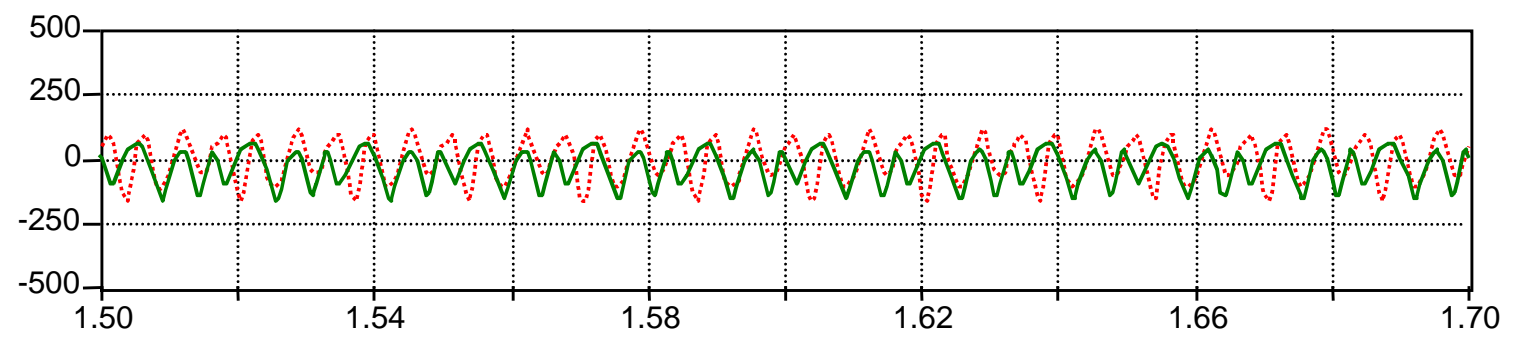

A15.ADF: Ch15

B1.pl4: c:IVHLSC-IVHBSC

dotted line: Fault Recorder solid line: Duality model

X-axis: Time in Secs, Y-axis: Voltage in Volt (Top), Current in Amperes (Bottom)

Figure 7.17 115-kV CB 5P147 C-phase Voltage (Top) and Current (Bottom) 1.5 seconds after Energization of $345-\mathrm{kV}$ Transformer No.9 at BLL 


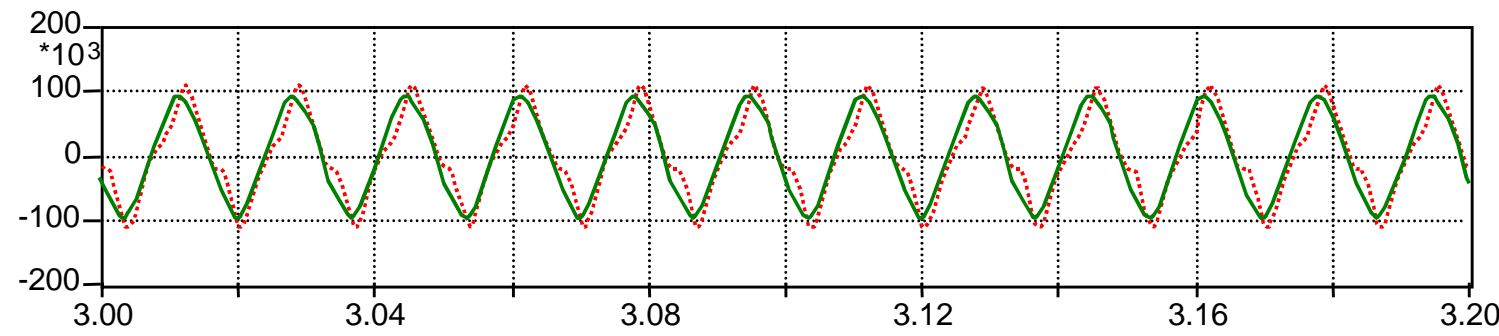

A3.ADF: Ch3

IVHGFR3B.pl4: v:IVHB1C

dotted line: Fault Recorder solid line: BCTRAN model

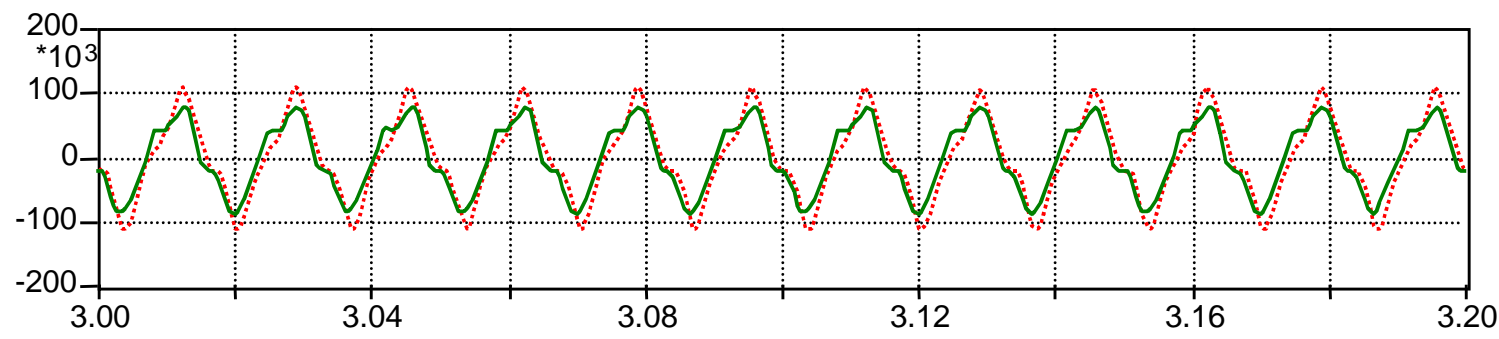

A3.ADF: Ch3

B1.pl4: v:IVHB1C

dotted line: Fault Recorder solid line: Duality model

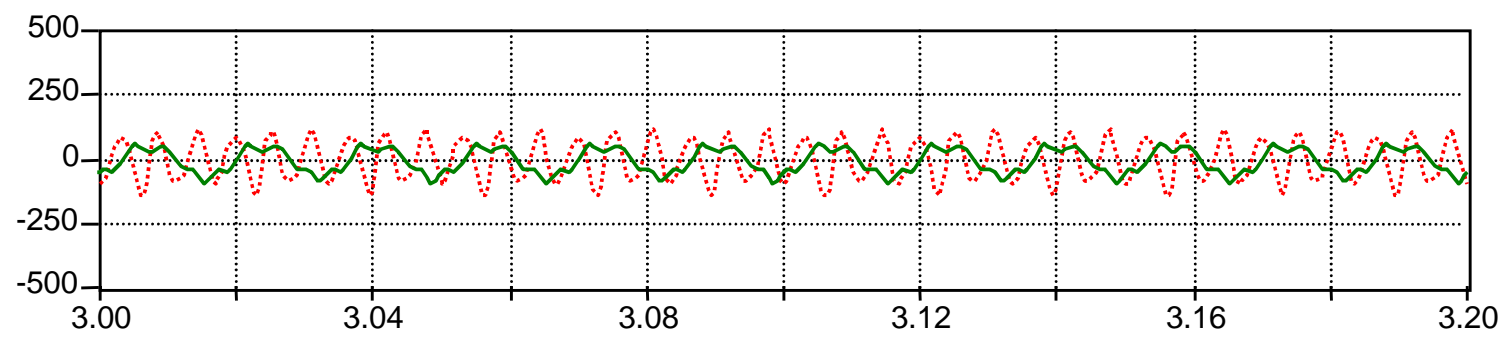

A15.ADF: Ch15

IVHGFR3B.pl4: c:IVHLSC-IVHBSC

dotted line: Fault Recorder solid line: BCTRAN model

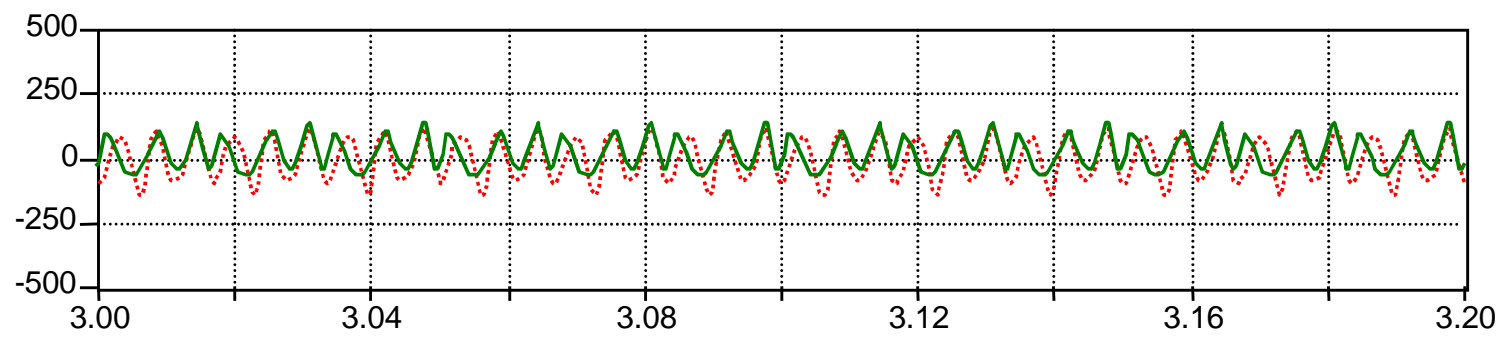

A15.ADF: Ch15

B1.pl4: c:IVHLSC-IVHBSC

dotted line: Fault Recorder solid line: Duality model

X-axis: Time in Secs, Y-axis: Voltage in Volt (Top), Current in Amperes (Bottom)

Figure 7.18 115-kV CB 5P147 C-phase Voltage (Top) and Current (Bottom)

3 seconds after Energization of 345-kV Transformer No.9 at BLL 


\section{CHAPTER 8}

\section{CONCLUSIONS AND RECOMMENDATIONS FOR FUTURE WORK}

The goal of this work was the development of duality-based transformer models and parameter estimation that can efficiently utilize available data and measurements that may be incomplete. Therefore, necessary parameters for duality based models, their interrelationships, and parameter estimation methods using optimization theory were studied to obtain proper model parameters.

This work extends the state of the art of topologically-correct three-phase autotransformer models and parameter estimation methods. Modeling results obtained from this work refine the nonlinear and frequency-dependent elements in the three-phase autotransformer equivalent circuit. Theoretical results obtained from this work provide a sound foundation for development of transformer parameter estimation methods using engineering optimization. In addition, it should be possible to refine which information and measurement data are necessary for complete duality-based transformer models. Simulation accuracy is dependent on the accuracy of the equipment model and its parameters. This work is significant in that it advances existing parameter estimation methods in cases where available data and measurements are incomplete. The accuracy of EMTP simulations for power systems including three-phase autotransformers is thus enhanced. 


\section{Conclusions}

In Chapter 4, parameters and characteristics of major components in equivalent circuits were refined. In order to improve the detailed representations used in transformer modeling, nonlinear and frequency-dependent characteristics were studied. Parameter estimation methods were developed to determine the parameters of a given model in cases where incomplete information is available.

1) Series Foster equivalent circuits with one cell give generally correct frequencydependent $\mathrm{R}$ in the given frequency range. However, a series Foster circuit with two cells was necessary for sufficiently accurate representation. Least square curve fitting methods gave proper parameters for the equivalent circuit.

2) Effective terminal capacitances determined by the frequency of TRV oscillations of each winding were within the reasonable ranges.

3) The Frolich equation used to model the core saturation curve gave a smooth single-valued anhysteretic curve and the obtained curves matched well with the nonlinear characteristic of the core.

4) Parameters for the transformer core loss model could be estimated using basic factory test data and optimization techniques. The eddy current loss could be modeled by a constant resistance. However, the current injection method should be used for modeling hysteresis loss because of its frequency-dependency.

5) The assumptions that the right displacement for each hysteresis loop is linear and the left displacement is nonlinear and increases slowly for low flux and more quickly for bigger flux, then decays to zero for maximum flux, were very effective and the obtained curves matched well with actual hysteresis loops.

In Chapter 5, duality-based equivalent circuit models for three-phase five-legged, three-legged, and shell-form autotransformers were developed for the EMTP implementation.

In Chapter 6, necessary parameters such as coil resistance, leakage inductance, core saturation component and core loss components were developed for the duality-based 
models in Chapter 5. Mathematical description of parameters and their interrelationships were refined.

1) When leakage inductances were derived from the basic physical structure and magnetic make-up of a three-winding transformer having cylindrical coils or pancake coils, there were many unknowns. Therefore, the ratios for winding width or winding area estimated from $R_{\mathrm{DC}}$ and the ratios for air-gap width estimated from voltage ratio were very useful.

2) The optimization technique was very effective in finding core saturation parameters. Forty points during each half cycle were necessary for the accuracy of RMS current calculation, since the current waveforms are not sinusoidal. More than forty points gave essentially the same results.

3) The DC hysteresis loop and eddy current loss of the core could be modeled using a Type-60 current source controlled by TACS in ATP. TACS was effective to incorporate the hysteresis loop model.

In Chapter 7, Steady-state excitation, de-energization, and re-energization transients were simulated and compared with the existing BCTRAN model. Black start energization cases were also simulated as a means of model evaluation and compared with actual event records. The simulated results using the model developed here were reasonable and more correct than those of the BCTRAN model.

\section{Suggestions for Further Study}

This work should be extended in the following ways:

- To further refine and develop the models and transformer parameter estimation methods developed here, iterative full-scale laboratory tests using high-voltage and high-power three-phase transformers would be helpful.

- The hysteresis loop model should be further studied for transients in case where the sign of the flux is changed before the reversing point of flux linkages. 
- The reactances for inner windings are negative. Physically, the negative reactance terms are a result of coil thickness and sometimes caused numerical instability. Therefore, newer short-circuit models without the negative reactance should be studied to enhance in the stability of simulation.

- The Frolich equation for core saturation modeling has limitations in flux density. Thus, newer equations for core saturation modeling might be developed.

- The parameter estimation techniques for frequency-dependent coil resistance and winding capacitances should be further refined, improving on the approach of using typical values.

- The third inductance, $\mathrm{L}_{3}$ in the frequency-dependent coil resistance model is a negative inductance. This negative inductance may give a numerical stability problem in ATP simulation. Therefore, incorporating all circuit components for Foster equivalent circuit into the leakage inductance matrix may yield a net positive inductance. This should be further studied to improve numerical stability.

- Core saturation modeling should be further studied for cases where the B-H curve of the core material is known or more excitation data than two points are available from the factory test report. 


\section{REFERENCES}

[1] ANSI/IEEE C37.011-1994, Guide for Transient Recovery Voltage for AC HighVoltage Circuit Breakers Rated on a Symmetrical current basis

[2] ANSI/IEEE C57.12.90-1999, Standard Test Code for Liquid-Immersed Dirstribution, Power, and Regulating Transformers

[3] Armco Catalog, "Oriented and TRAN-COR H Electrical Steels", 10th Edition, Jan. 1986.

[4] C.M. Arturi, "Transient Simulation and Analysis of a Five-Limb Step-Up Transformer Following and Out-of-Phase Synchronization,” IEEE Transactions on Power Delivery, Vol. 6, No. 1, January 1991, pp. 196-207.

[5] Michael A. Bjorge, Investigation of Short-Circuit Models for A four-Winding Transformer, M.S. Thesis, Michigan Technological University, 1996.

[6] M.H.J. Bollen, "The Search for a General Transformer Model," $16^{\text {th }}$ European EMTP Users Group Meeting, paper 89-07, May 28-30, 1989, pp. 1-20.

[7] Richard .L. Bean, Nicholas Chackan Jr, Harold R. Moore, and Edward C. Wentz, Transformers for the Electric Power Industry, McGraw-Hill Book Company, 1959.

[8] V. Brandwajn, H.W. Dommel, and I.I. Dommel, "Matrix Representation of ThreePhase N-Winding Transformers for Steady-State and Transient Studies," IEEE Transactions on Power Apparatus and Systems, Vol. PAS-101, No. 6, June 1982, pp. 1369-1378.

[9] Xusheng Chen and S.S. Venkata, "A Three-Phase Three-Winding Core-Type Transformer Model for Low-Frequency Transient Studies," IEEE Transactions on Power Delivery, Vol. 12, No. 2, April 1997, pp. 775-782.

[10] Xusheng Chen, "Negative Inductance and Numerical Instability of the Saturable Transformer Component in EMTP," IEEE Transactions on Power Delivery, Vol. 15, No. 4, October 2000, pp. 1199-1204.

[11] Xusheng Chen, "Final Report: The Development of a Three-Phase Multi-Legged Transformer Model for Use with EMTP”, Bonneville Power Administration Award No. DE-AC79-92BP26702, 1993.

[12] Xusheng Chen, "A three-phase multi-legged transformer model in ATP using the directly-formed inverse inductance matrix”, IEEE Transactions on Power Delivery, Vol. 11 Issue: 3 , July 1996, pp. $1554-1562$. 
[13] E. Collin Cherry, "The Duality Between Interlinked Electric and Magnetic Circuits and the Formation of Transformer Equivalent Circuits," Proceedings of the Physical Society, Part B, Vol. 62, 1949, pp. 101-111.

[14] Sung D. Cho, Bruce A. Mork, Kalyan K. Mustaphi, “ Inver Hills Black Start Supplemental EMTP Investigation (The Step-by-step Switching Cases)”, internal research report, Xcel Energy, Minneapolis, MN, May 14, 2001.

[15] Sung D. Cho, Bruce A. Mork, Kalyan K. Mustaphi, "Transient and harmonic overvoltages during black start energization”, 37th Annual Minnesota Power Systems Conference, November 12, 2001.

[16] Abhijit C. Devasthale, Transient Modeling of Three-Legged Amorphous Core Transformer, M.S. Thesis, Michigan Technological University, 1996.

[17] H.W. Dommel with S. Bhattacharya, V. Brandwajn, H.K. Lauw and L. Martí, Electromagnetic Transients Program Reference Manual (EMTP Theory Book), Bonneville Power Administration, Portland, USA, August 1986.

[18] O. Einarsson, "EMTP: Art of Modelling," ASEA Internal Technical Report, RM KZEB 87-010, July 7, 1987, pp. 1-20, attachments 1-6.

[19] W. Enright, O.B. Nayak, G.D. Irwin, and J. Arrillaga, "An Electromagnetic Transients Model of Multi-Limb Transformers Using Normalized Core Concept," International Conference on Power Systems Transients IPST'97, Seattle, USA, June 22-26, 1997.

[20] W. Enright, N. Watson, and O.B. Nayak, "Three-Phase Five-Limb Unified Magnetic Equivalent Circuit Transformer Models for PSCAD V3, " International Conference on Power Systems Transients IPST'99, Budapest, Hungary, June 20-24, 1999.

[21] J.G. Frame, N. Mohan, and T. Liu, "Hysteresis Modeling in an Electromagnetic Transients Program," IEEE Transactions on Power Apparatus and Systems, Vol. PAS-101, No.9, September 1982, pp. 3403-3411.

[22] M. J. Heathcote, The J\&P Transformer Book - 12 $2^{\text {th }}$ Edition, Newnes Ltd., 1998.

[23] M.J. Gaffney, Amorphous Core Transformer Model for Transient Simulation, M.S. Thesis, Michigan Technological University, August 1996.

[24] Allan Greenwood, Electrical Transients in Power Systems, John Wiley \& Sons, Inc., 2nd edition, 1991.

[25 ] David C. Greyerbiehl, Development and Investigation of Anhysteretic Magnetic Core Models, M.S. Thesis, Michigan Technological University, 1998. 
[26] R.H. Harner and J. Rodriguez, "Transient Recovery Voltage Associated with Power-System Three-Phase Transformer Secondary Faults", IEEE Trans. PAS, Vol. PAS-91, No.5, Sep./Oct. 1972, pp. 1887-1896.

[27] N.D. Hatziargyriou, J.M. Prousalidis, and B.C. Papadias, "Generalised Transformer Model Based on the Analysis of its Magnetic Core Circuit," IEE Proceedings-C, Vol. 140, No. 4, July 1993, pp. 269-278.

[28] F.D. Leon, A. Semlyen, "Time Domain Modeling of Eddy Current Effects for Transformer Transients", IEEE Transactions on Power Delivery, Vol. 8, No. 1, January 1993, pp. 271-280.

[29] F.D. Leon, A. Semlyen, "Detailed Modeling of Eddy Current Effects for Transformer Transients”, IEEE Transactions on Power Delivery, Vol. 9, No. 2, April 1994, pp. 1143-1150.

[30] K.U. Leuven EMTP Center, Alternative Transients Program Rule Book, Leuven EMTP Center, Heverlee, Belgium, July 1987.

[31] B.A. Mork, Ferroresonance and Chaos: Observation and Simulation of Ferroresonance in a Five-Legged Core Distribution Transformer, PhD Dissertation, North Dakota State University, 1992.

[32] B.A. Mork, "Five-legged Wound Core Transformer Model: Derivation, Parameters, Implementation, and Evaluation," IEEE Transactions on Power Delivery, Vol. 14, No. 4, October 1999, pp. 1519-1526.

[33] Arun Narang and Russell H. Brierley, "Topology Based Magnetic Model for Steady-State and Transients Studies for Three-Phase Core Type Transformers," IEEE/PES Summer Meeting, Vancouver, Canada, July 18-22, 1993.

[34] W.L.A. Neves and H.W. Dommel, "On Modeling Iron Core Nonlinearities," IEEE/PES Winter Meeting, New York, USA, January 26-30, 1992.

[35] W.L.A. Neves and H.W. Dommel, "Transformer Core Modelling," International Conference on Power Systems Transients IPST'95, Lisbon, Portugal, September 3-7, 1995.

[36] Kedar D. Pandit, Automated measurement of Transformer Core Parameters, M.S. Thesis, Michigan Technological University, 1995.

[37] B.K. Perkins, J.R. Martí, and H.W. Dommel, "Nonlinear Elements in the EMTP: Steady-State Initialization,” IEEE/PES Summer Meeting, Vancouver, Canada, July 18-22, 1993. 
[38] M. Popov, L. Van der Sluis, G.C. Paap, and P.H. Schavemaker, "On a Hysteresis Model for Transient Analysis,” IEEE Power Engineering Review, May 2000.

[39] Prashanth S.S. Holenarsipur, Ned Mohan, Vernon D. Albertson, and Jack Christofersen, "Avoiding the Use of Negative Inductances and Resistances in Modeling Three-Winding Transformers for Computer Simulations," IEEE/PES Winter Meeting, New York, USA, January 31-February 4, 1999.

[40] G.V. Reklaitis, A.Ravindran, K.M. Ragsdell, Engineeering Optimization, John Wiley and Sons, 1983

[41] C. Saldaña and G. Calzolari, "Analysis of Core Type Transformer Models Based on the Principle of Duality in Electromagnetic Transients Studies," International Conference on Power Systems Transients IPST'97, Seattle, USA, June 22-26, 1997.

[42] A.K. Sawhney, A Course in Electric Machine Design, pp. 406-411, 8th edition, J.C. Kapur for Rai and Sons, Delhi-Jullundur, Reprint 1987, copyright 1945.

[43] A. Schellmanns, P. Fouassier, JP. Keradec, JL. Schanen, “1D-Propagation based Equivalent Circuit for Transformers: Accouting for Multi-Layer structure of Widnings and Ferrite Losses”, IEEE Industry Application Society $32^{\text {nd }}$ Annual Meeting, October 1997, pp. 1211-1216.

[44] A. Schellmanns, J.P. Keradec, J.L. Schannen, "Electrical Equivalent Circuit for Frequency Dependent Impedance: Minimum Lumped Elements for a Given Precision”, IEEE, Industry Applications Conference, Volume 5, 2000

[45] A. Semlyen, F.D. Leon, "Eddy Current Add-on for Frequency Dependent Represenation of Winding Losses in Transformer Models used in Computing Electromagnetic Trasients”, IEE Proc. Gener,Transm. Distrib., Vol.141, No.3, May 1994, pp. 209-213.

[46] G.R. Slemon, "Equivalent Circuits for Transformers and Machines Including NonLinear Effects", Proceedings Institution of Electrical Engineers, Vol. 100, Part IV, 1953, pp. 129-143.

[47] Don L. Stuehm, "Final Report: Three-Phase Transformer Core Modeling", Bonneville Power Administration Award No. DE-BI79-92BP26700, Feb. 1993

[48] P. E. Sutherland, "Modeling of Impedance vs. Frequency in Harmonic Analysis Programs”, IEEE Industry Application Society Annual Meeting, October 1997, pp. 2243-2247.

[49] Julio Usaola and Guido Empereur, "Comparison Between Different Transformer Models in EMTP,” EMTP News, Vol. 2, No. 2, June 1989, pp. 25-34. 
[50] IEEE Working Group 15.08.09, "Tutorial on Modeling and Analysis of system Transients using Digital Program”, IEEE PES Special Publication, TP-133-0, Chapter 3 and 4, 1999.

[51] Working Group C-5 of the Systems Protection Subcommittee, Tziouvaras, D.A.; McLaren, P.; Alexander, G.; Dawson, D.; Esztergalyos, J.; Fromen, C.; Glinkowski, M.; Hasenwinkle, I.; Kezunovic, M.; Kojovic, L.; Kotheimer, B.; Kuffel, R.; Nordstrom, J.; Zocholl, S., "Mathematical models for current, voltage, and coupling capacitor voltage transformers", IEEE Transactions on Power Delivery, Vol. 15 Issue: 1 , Jan. 2000, pp. $62-72$.

[52] CIGRE Working Group 02(SC33), "Guidelines for Representation of Network Elements when calculating Transients”, 1990.

[53] The Mathworks Inc., User's Guide Version 2 for Optimization Toolbox, 1999.

[54] Orland P. Hevia, "Hysteresis Hevia: a new routine to generate input data for inductors with hysteresis”, EEUG News, Feb.- May, 2000. 


\section{APPENDIX A: SAMPLE ATP DATA FILE}

\section{Appendix A.1: CSLBS.ATP}

The steady-state simulation for duality model of shell-form transformer

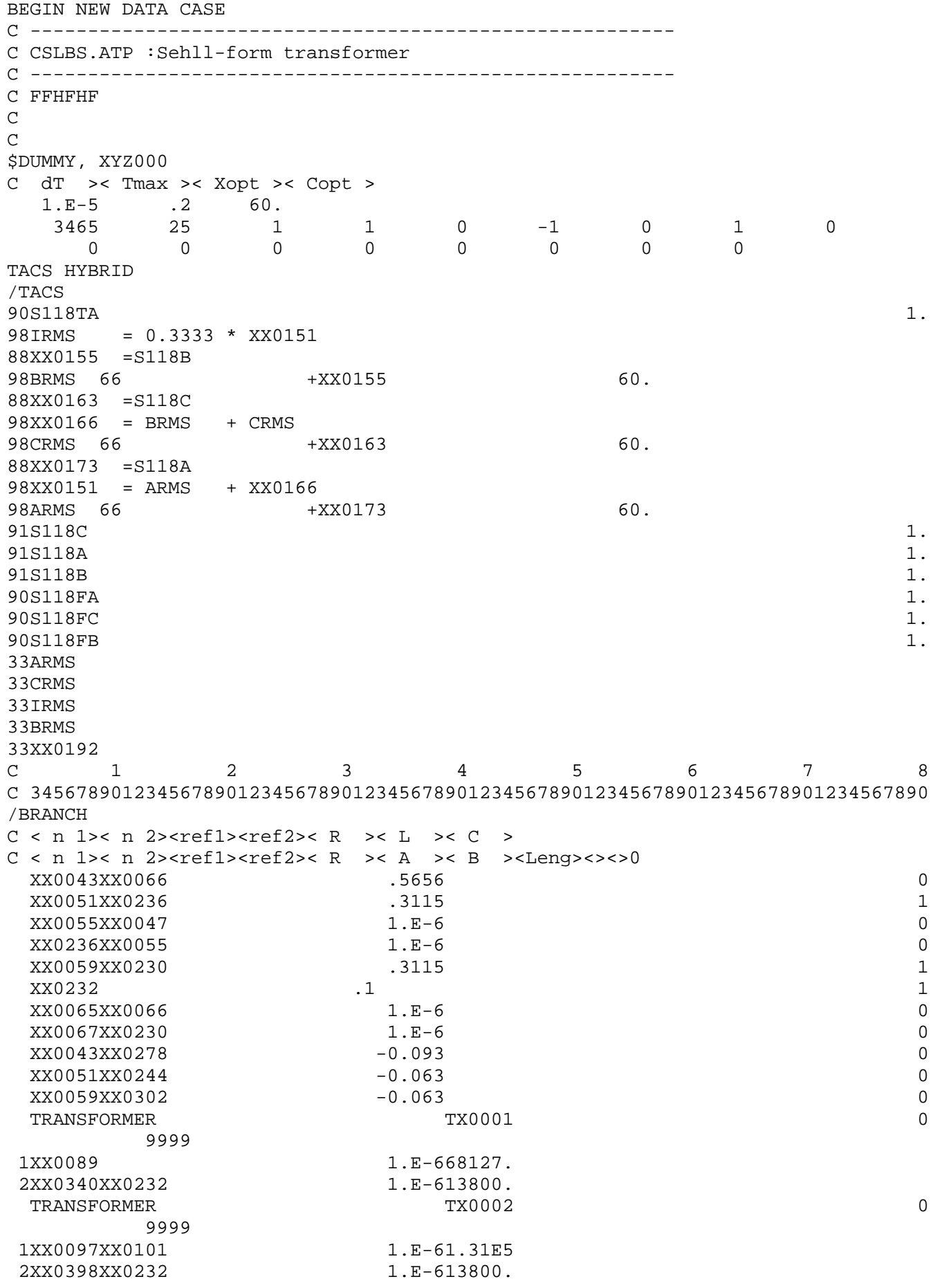

$98 \times X 0151=$ ARMS
98 ARMS 66

6.

0. 


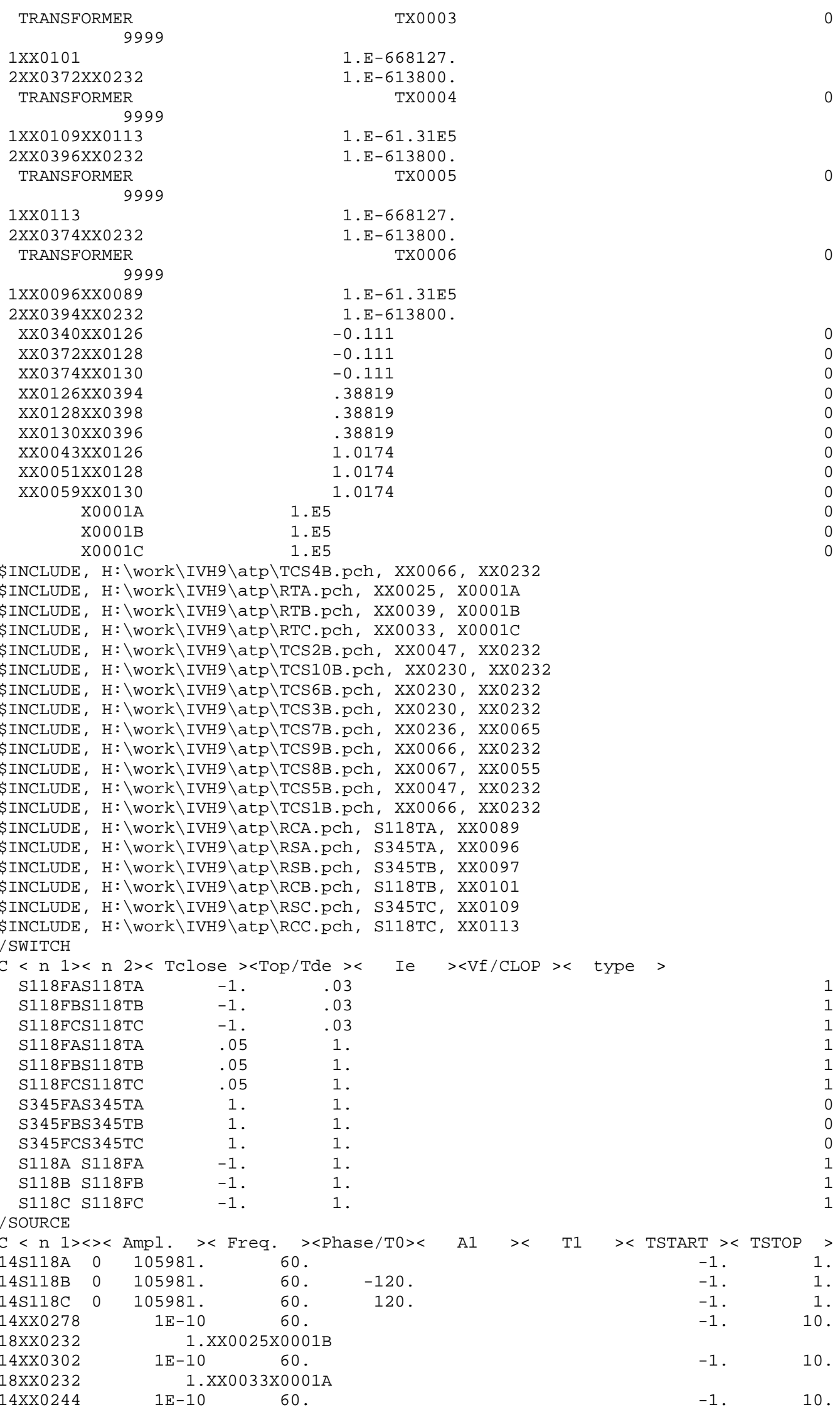




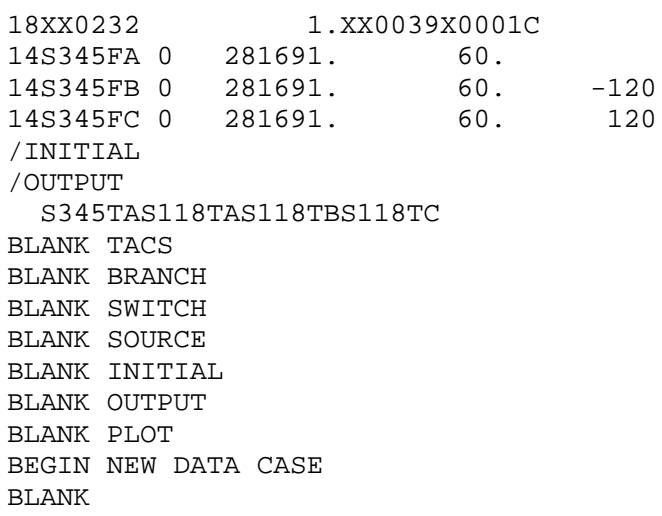

\section{Appendix A.2: TCS1B.PCH}

\section{TACS model for core section No.1 of shell-form transformer}

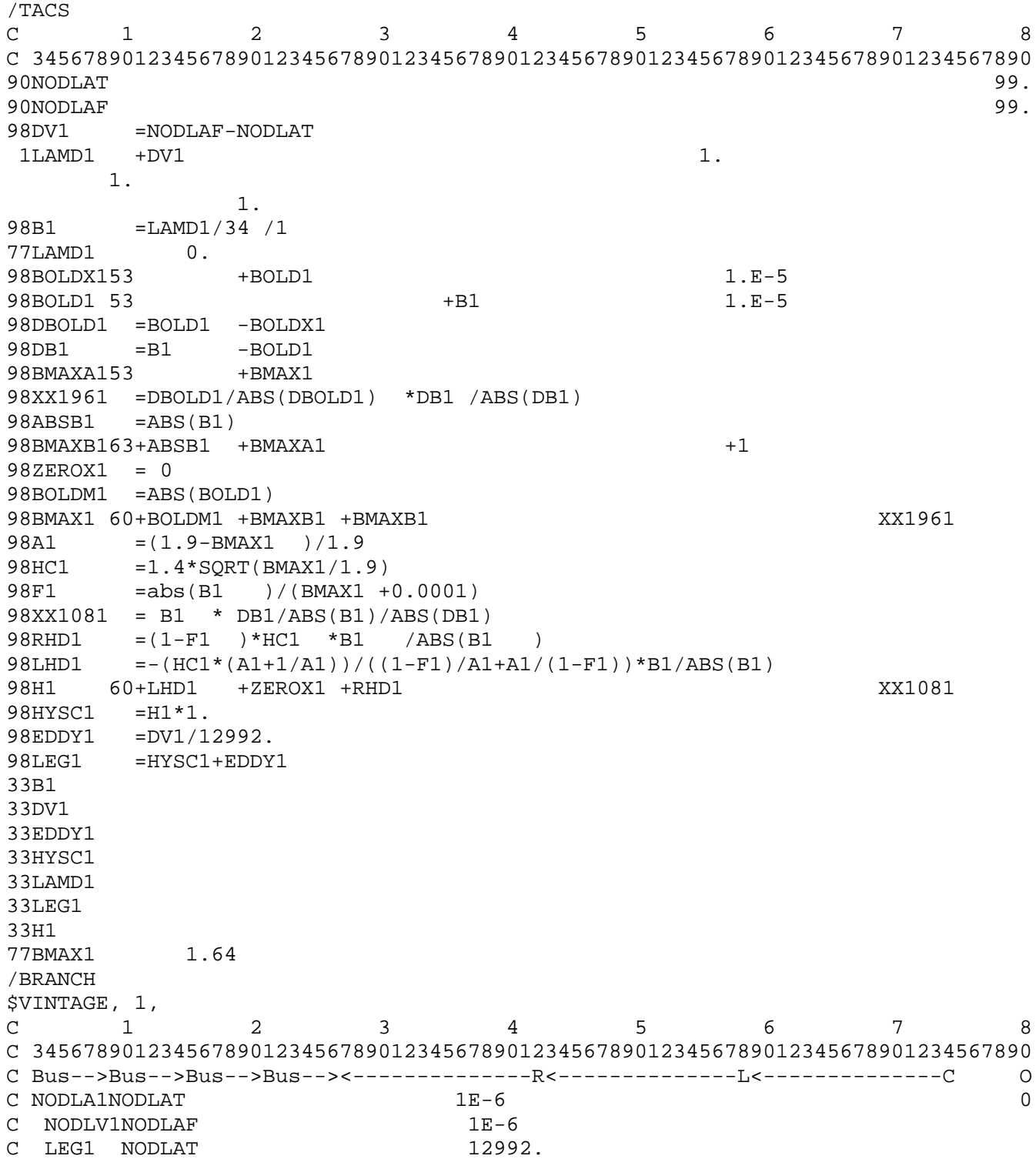




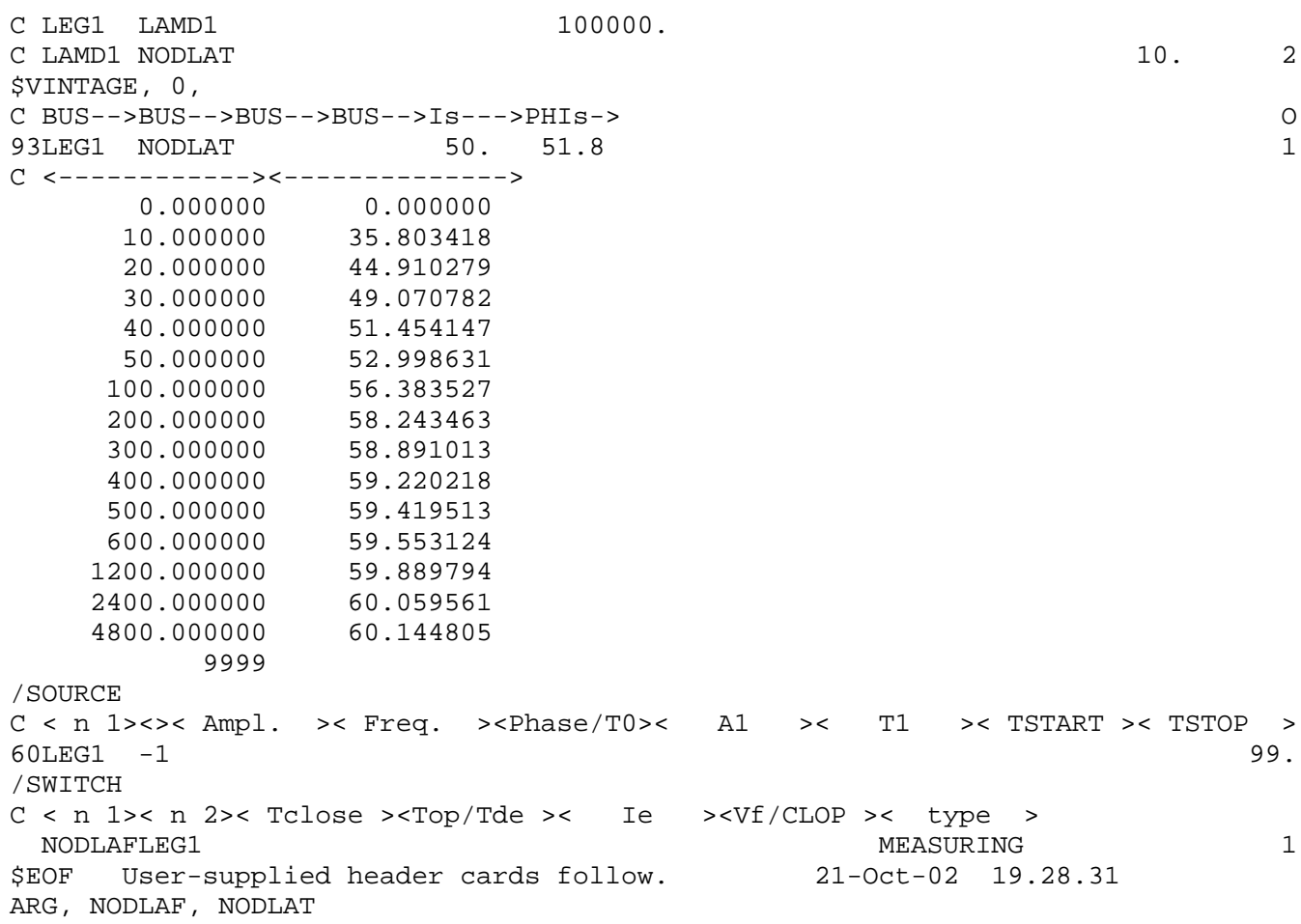

Appendix A.3: TCS2B.PCH

TACS model for core section No.2 of shell-form transformer

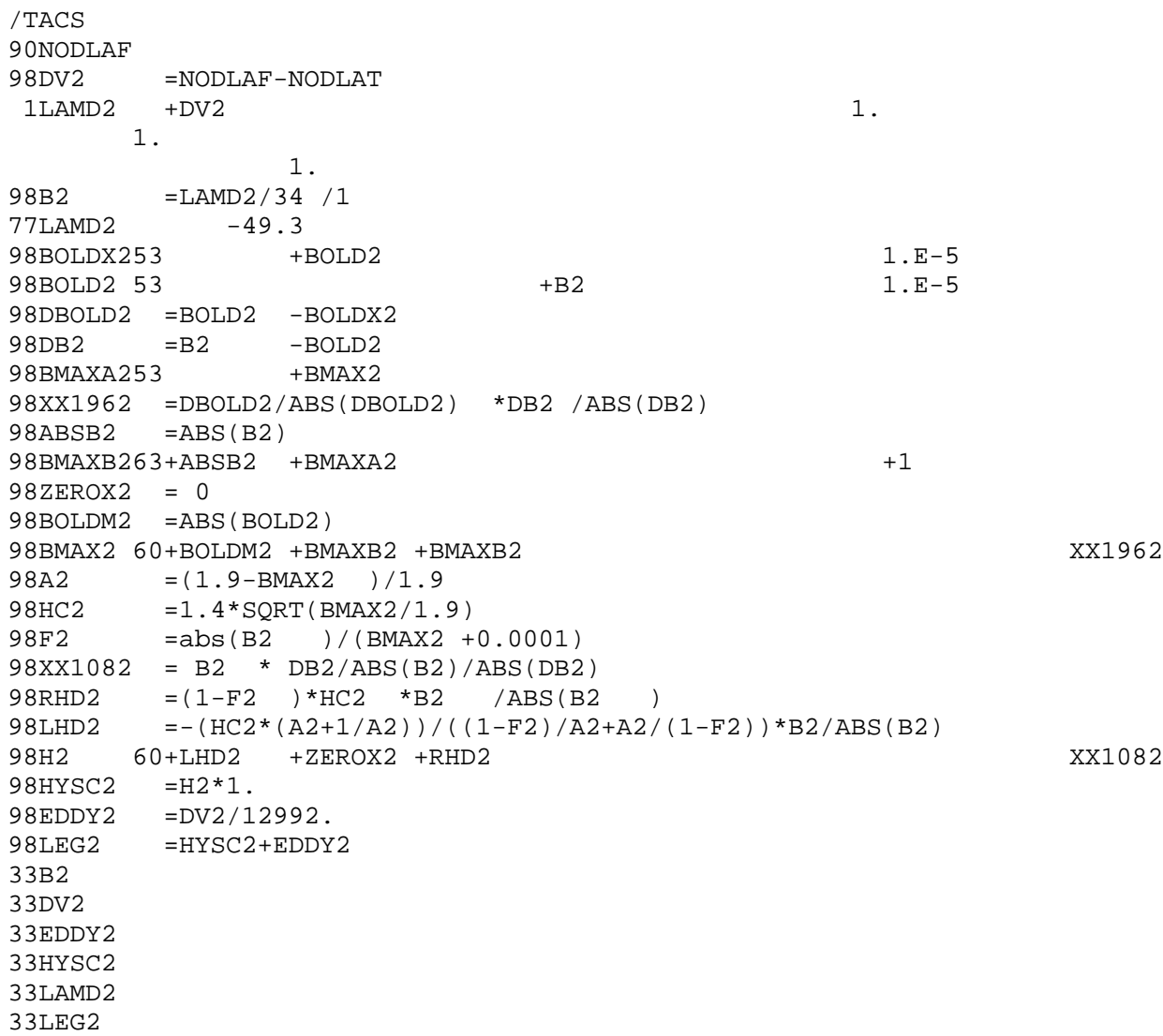

98BMAX2 60+BOLDM2 +BMAXB2 +BMAXB2 2

1 . 1 


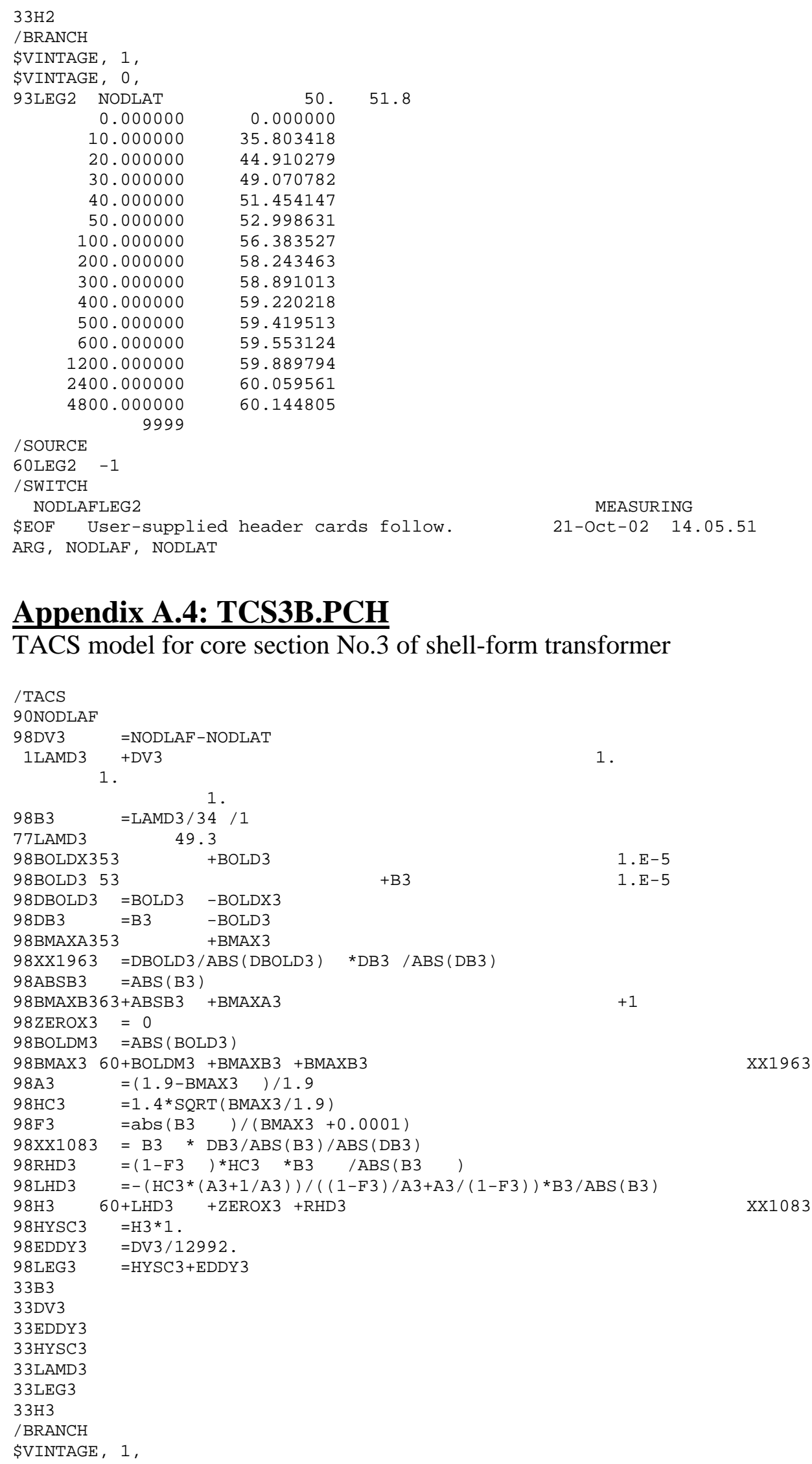

\section{Appendix A.4: TCS3B.PCH}

\section{TACS model for core section No.3 of shell-form transformer}




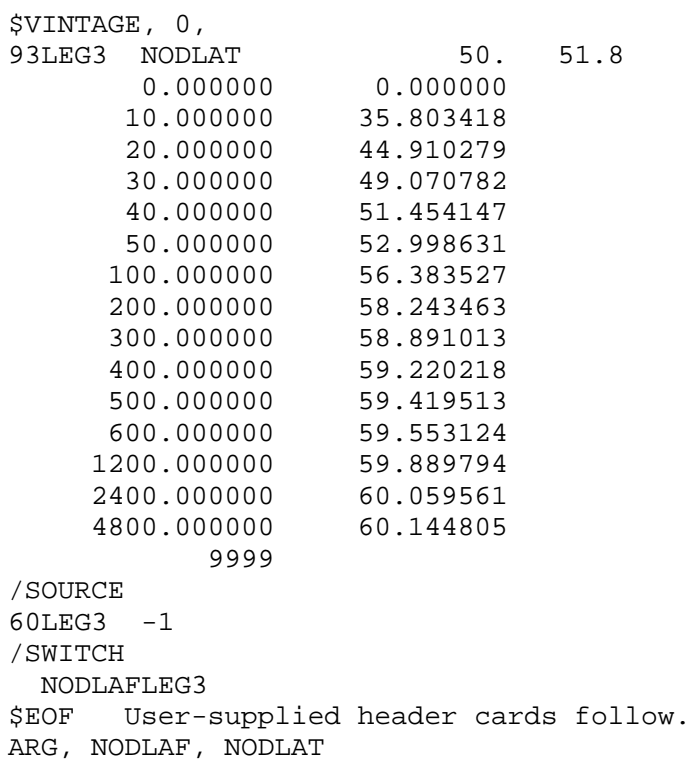

\section{Appendix A.5: TCS4B.PCH}

TACS model for core section No.4 of shell-form transformer

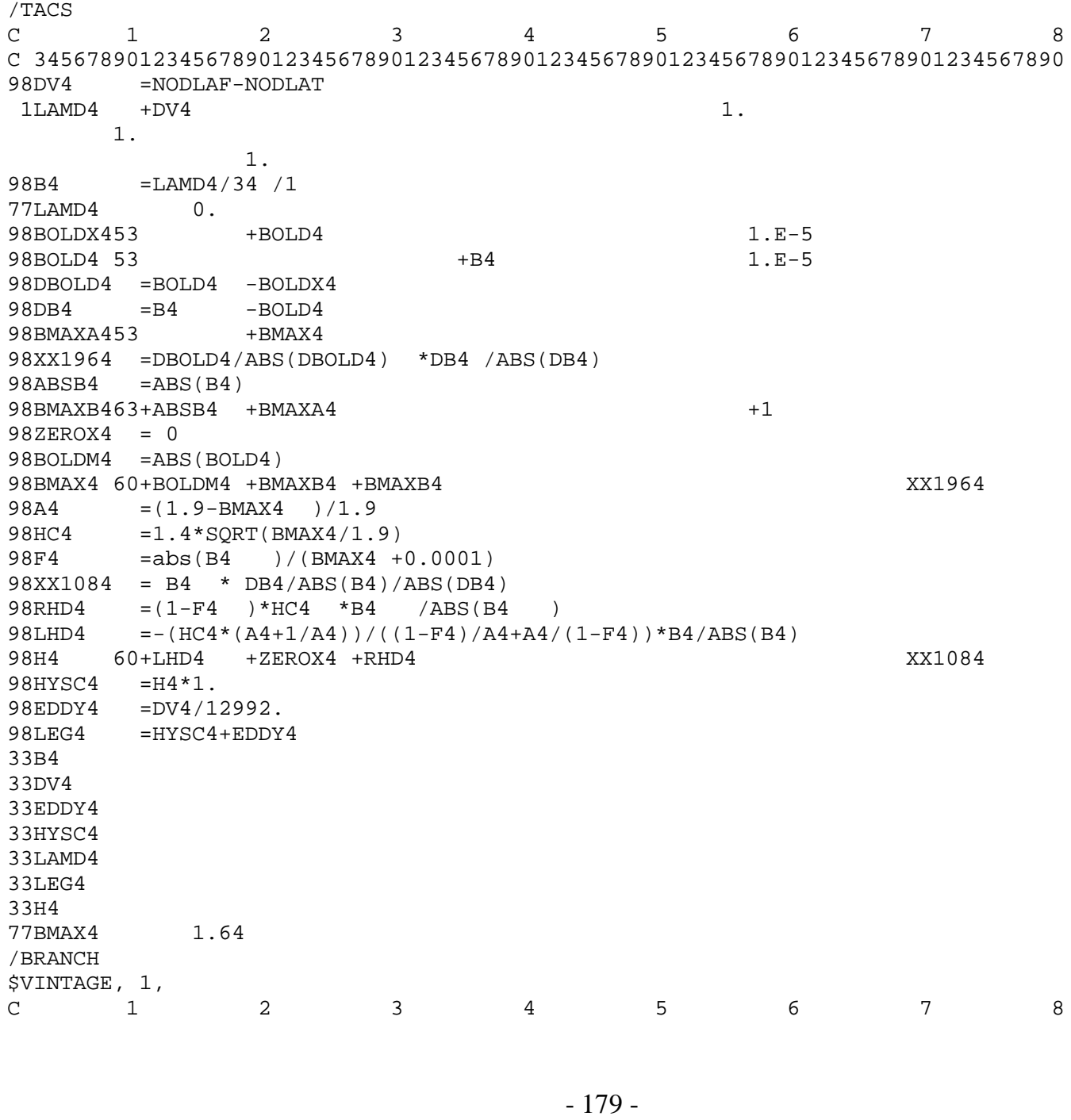

23

34

45

56

6 


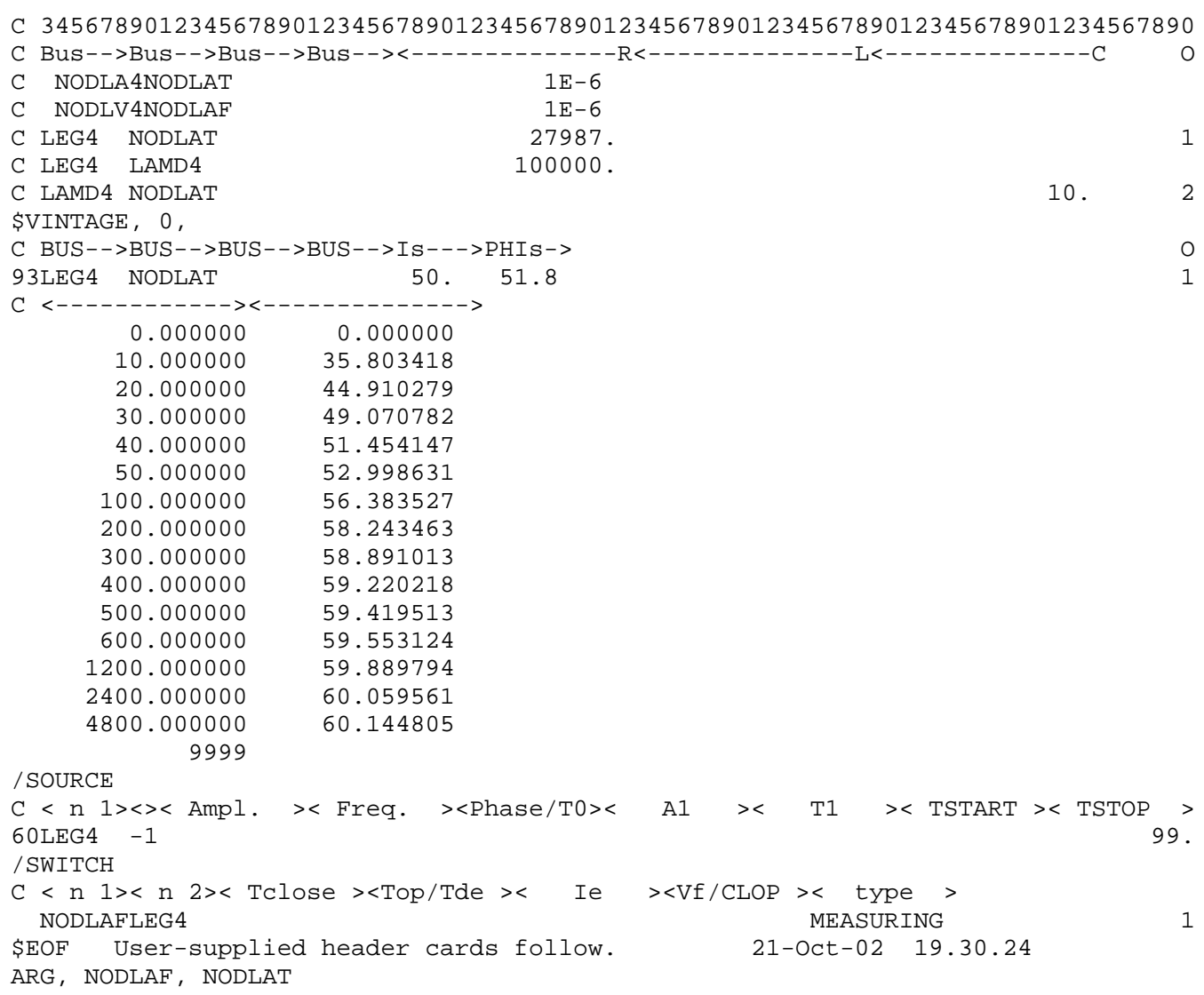

\section{Appendix A.6: TCS5B.PCH}

TACS model for core section No.5 of shell-form transformer

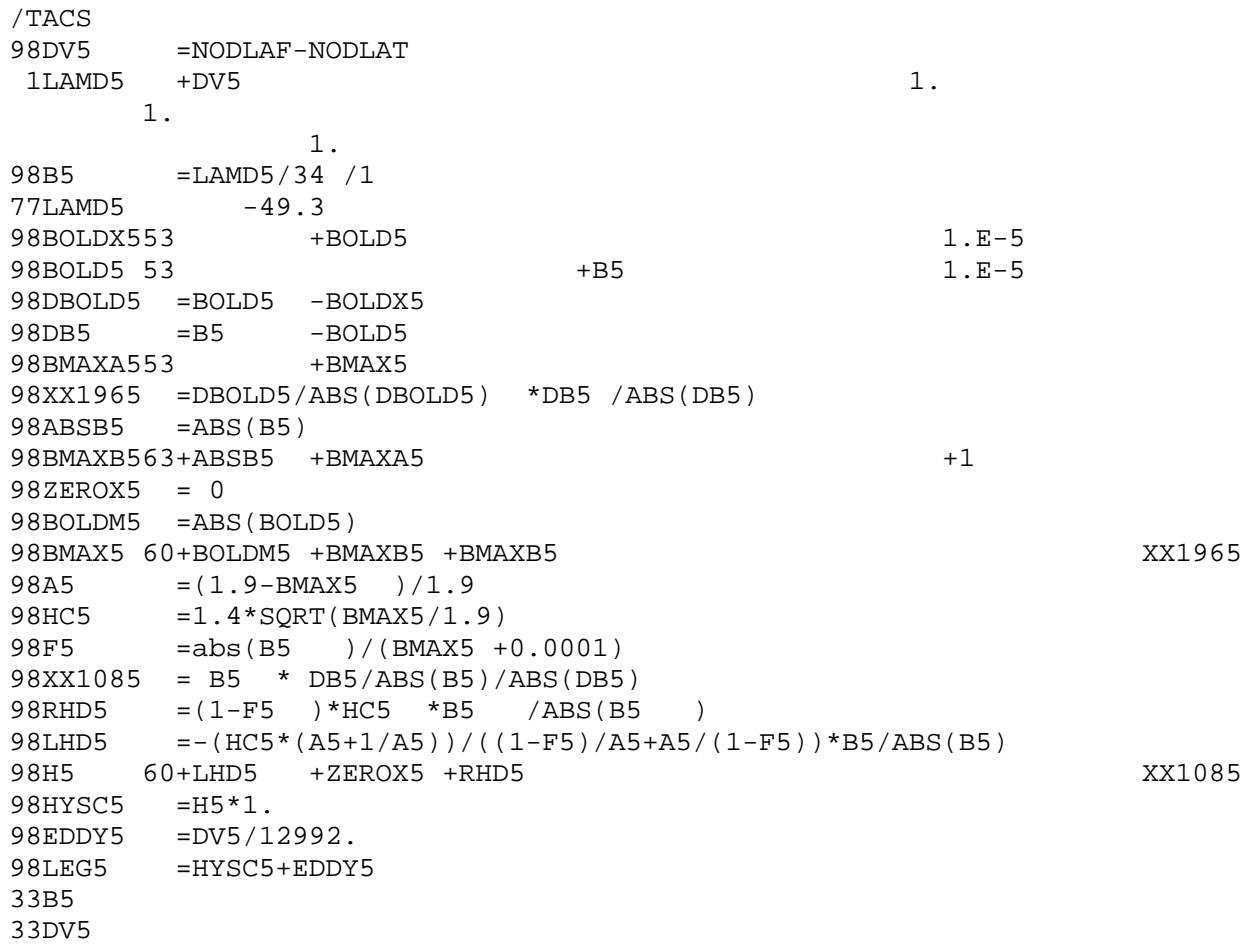

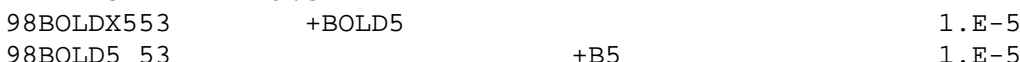
98 BMAXB563+ABSB5 +BMAXA5 +1

98BMAX5 60+BOLDM5 +BMAXB5 +BMAXB5

1.

XX1085 
20.000000

0.000000

35.803418

44.910279

$30.000000 \quad 49.070782$

$40.000000 \quad 51.454147$

$50.000000 \quad 52.998631$

$100.000000 \quad 56.383527$

$200.000000 \quad 58.243463$

$300.000000 \quad 58.891013$

$400.000000 \quad 59.220218$

$500.000000 \quad 59.419513$

$600.000000 \quad 59.553124$

$1200.000000 \quad 59.889794$

$2400.000000 \quad 60.059561$

$4800.000000 \quad 60.144805$

/SOURCE

60LEG5 -1

9999

/SWITCH

NODLAF LEG5

\$EOF User-supplied header cards follow.

$A R G$, NODLAF, NODLAT

99.

MEASURING

1

\section{Appendix A.7: TCS6B.PCH}

TACS model for core section No.6 of shell-form transformer

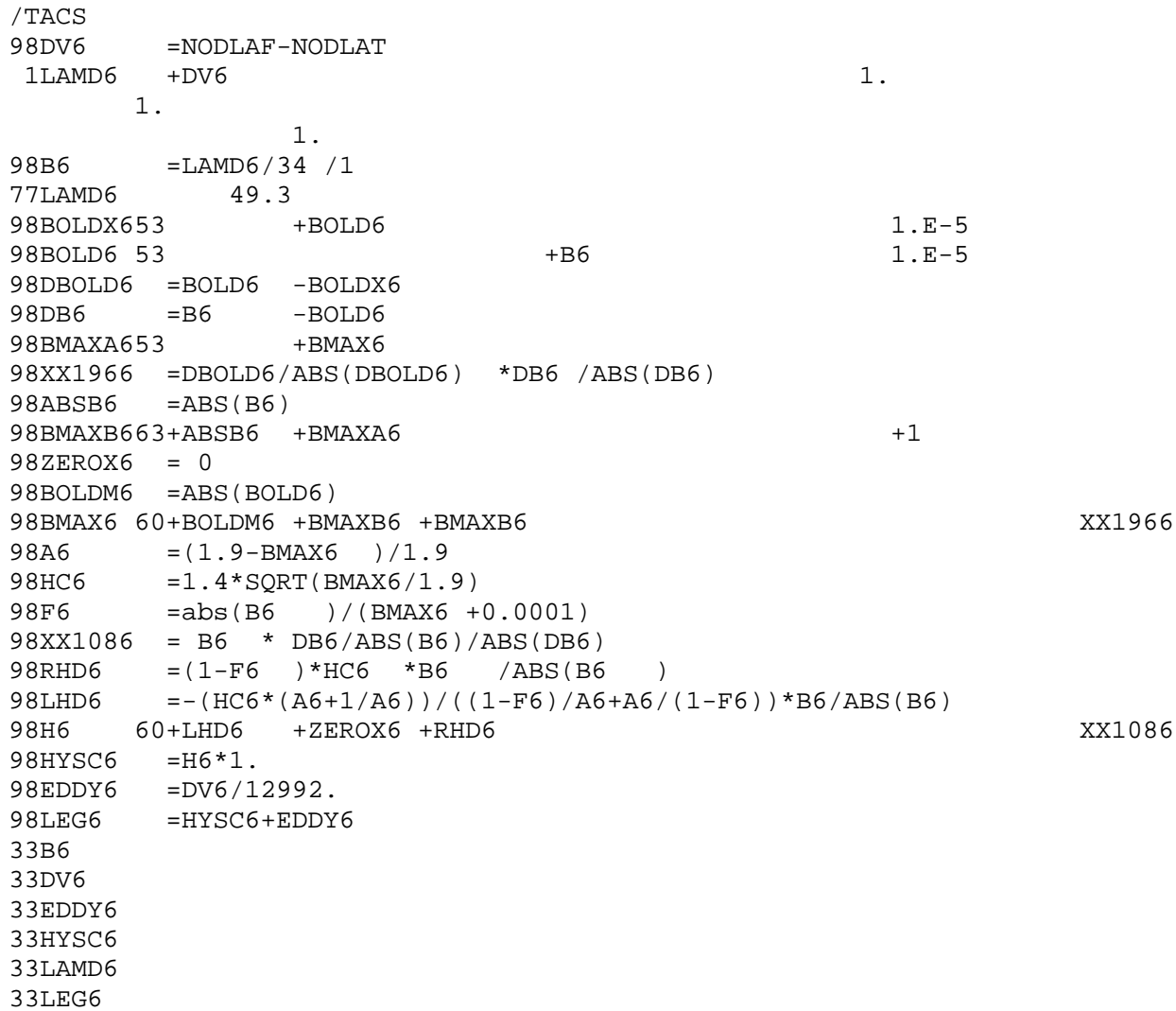




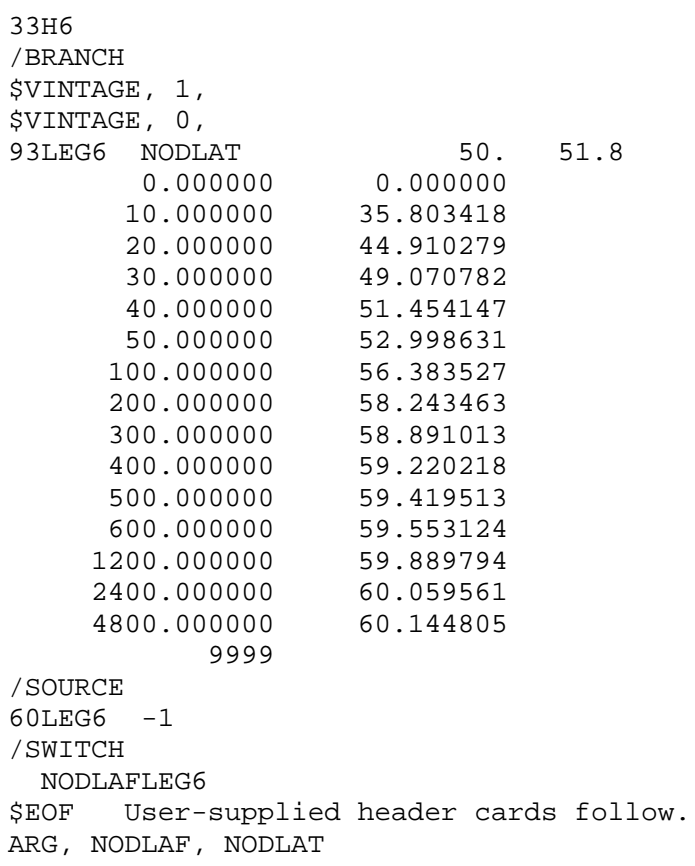

\section{Appendix A.8: TCS7B.PCH}

\section{TACS model for core section No.7 of shell-form transformer}

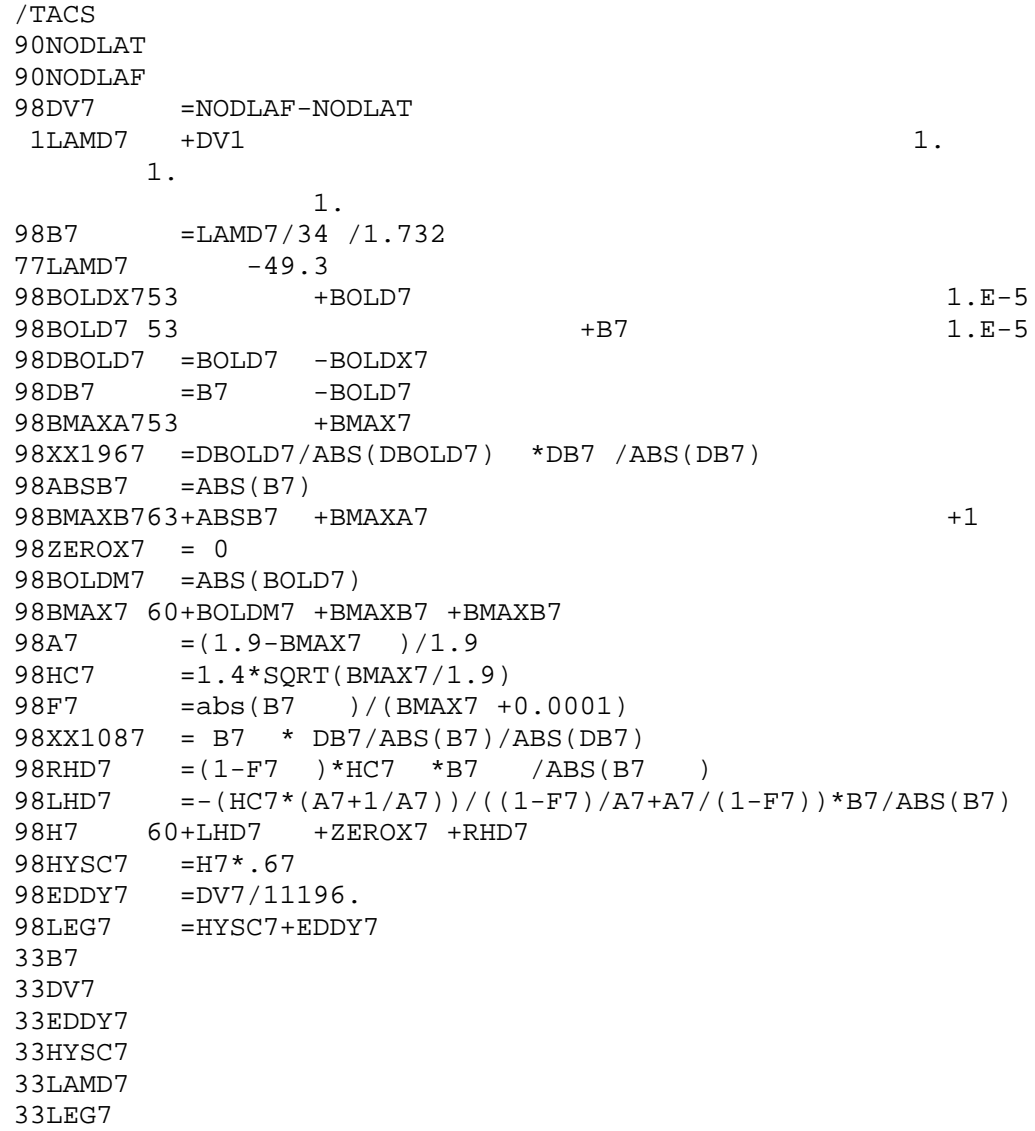




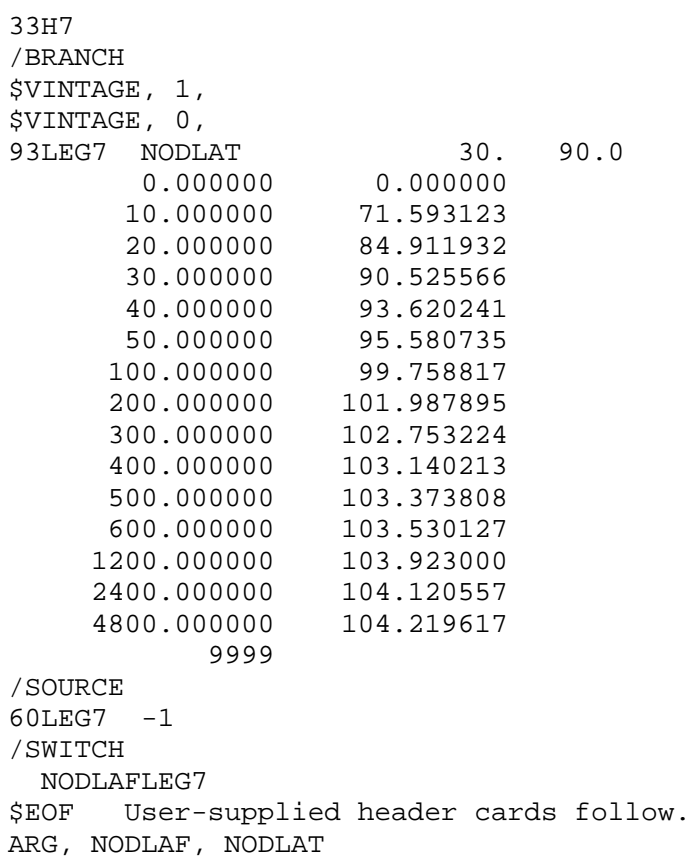

\section{Appendix A.9: TCS8B.PCH}

TACS model for core section No.8 of shell-form transformer

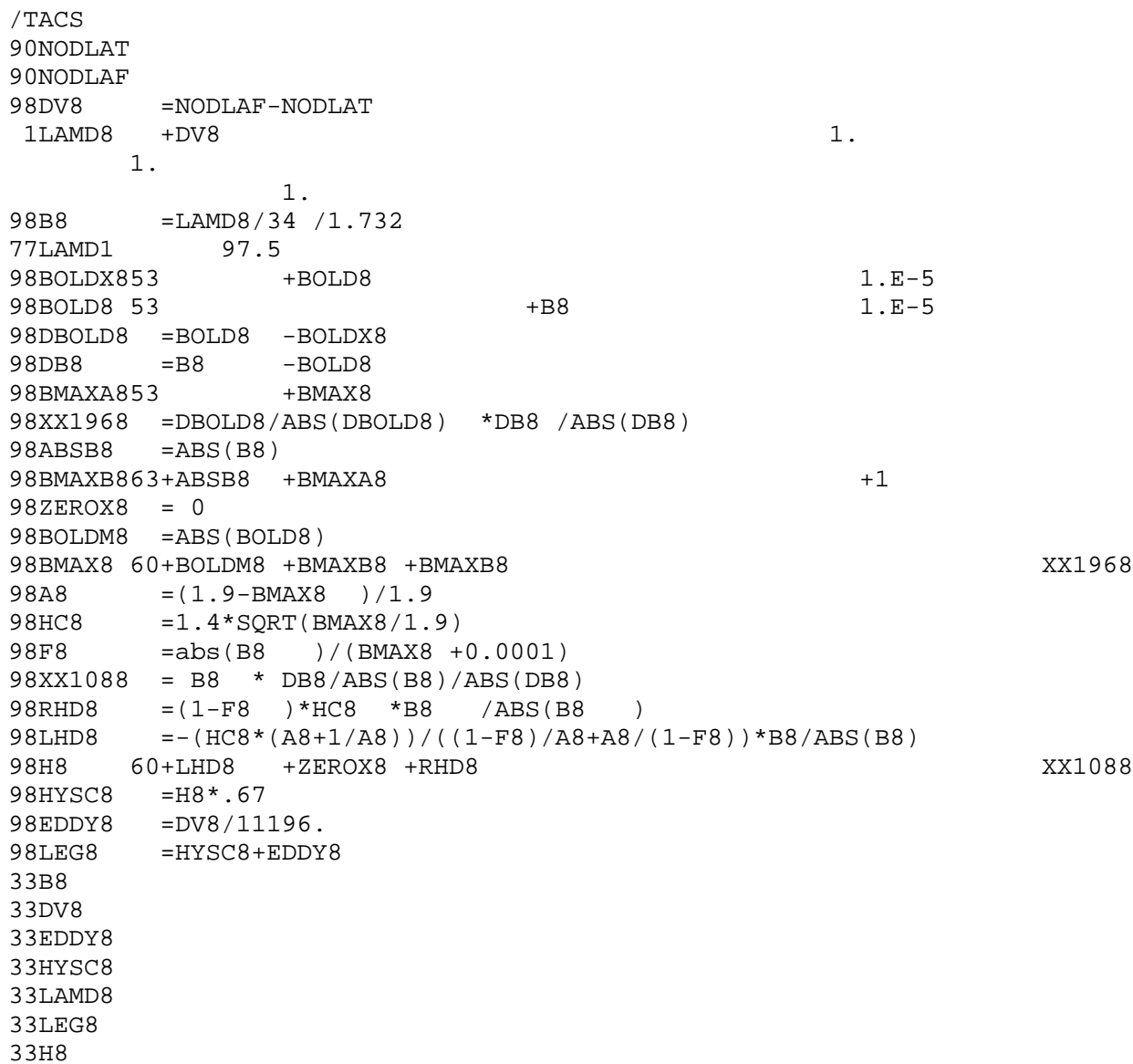




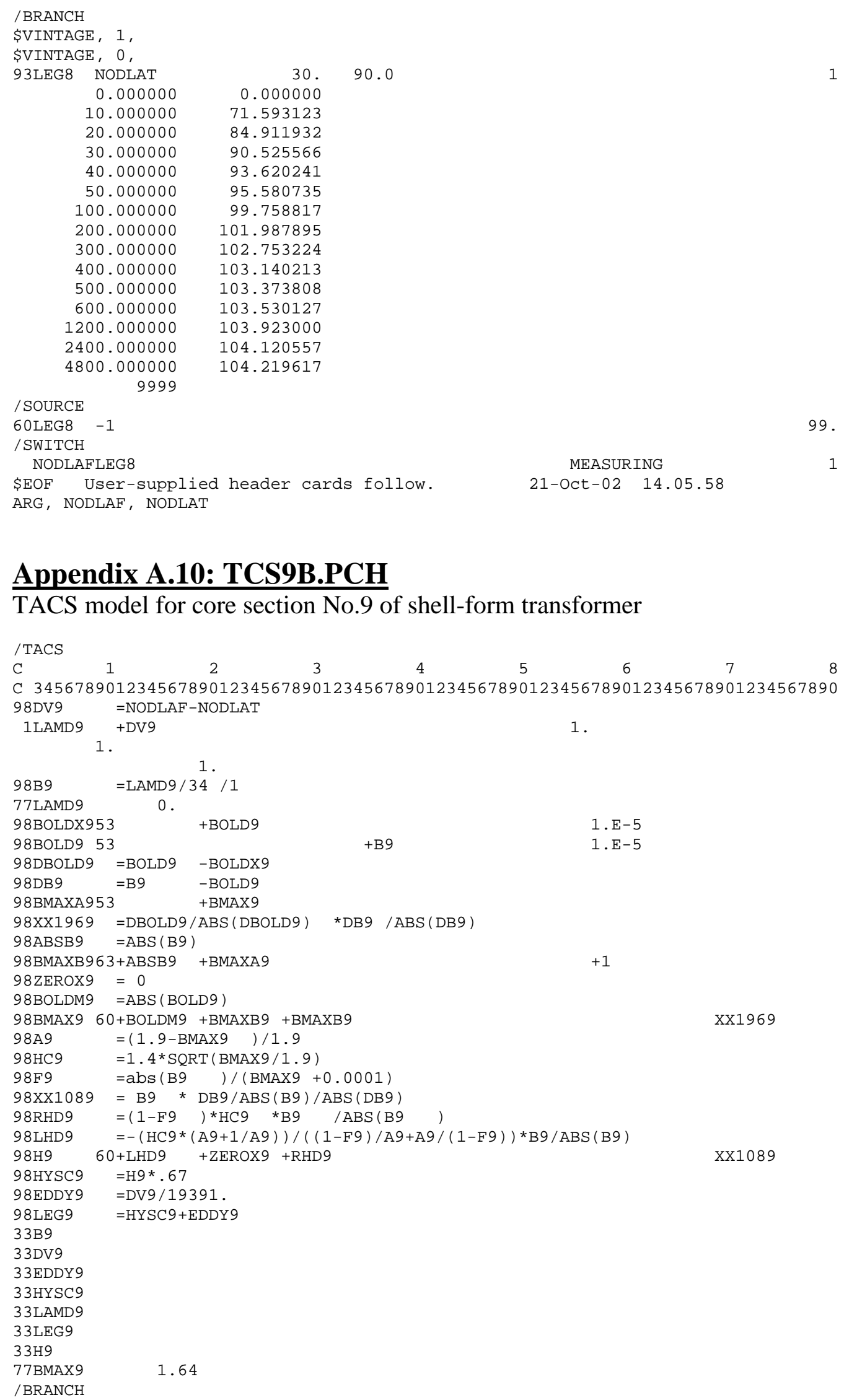

MEASURING $21-$ Oct-02 14.05 .58

99.

\section{Appendix A.10: TCS9B.PCH}

\section{TACS model for core section No.9 of shell-form transformer}




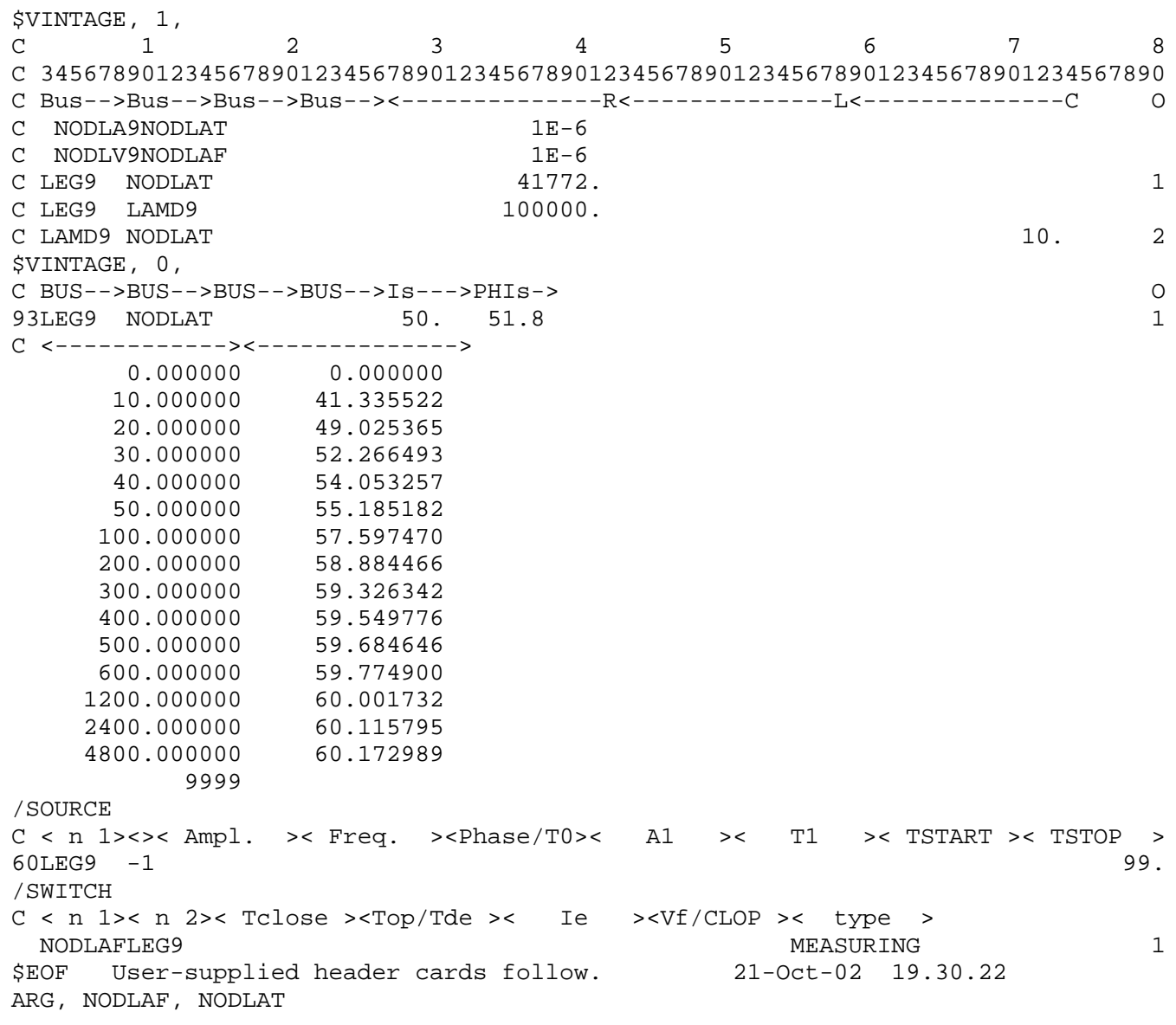

\section{Appendix A.11: TCSVB.PCH}

TACS model for core section No.10 of shell-form transformer

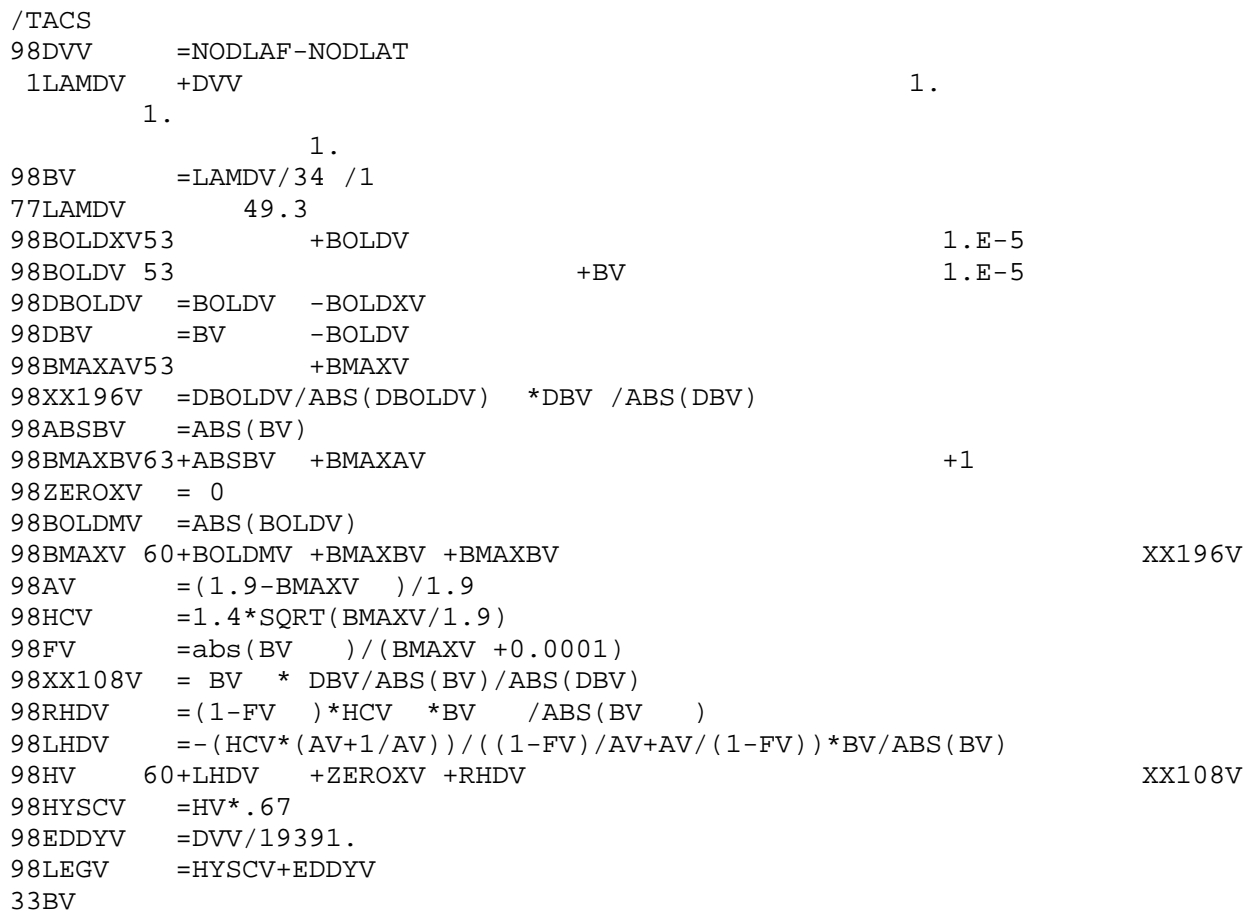


$20.000000 \quad 49.025365$

$30.000000 \quad 52.266493$

$40.000000 \quad 54.053257$

$50.000000 \quad 55.185182$

$100.000000 \quad 57.597470$

$200.000000 \quad 58.884466$

$300.000000 \quad 59.326342$

$400.000000 \quad 59.549776$

$500.000000 \quad 59.684646$

$600.000000 \quad 59.774900$

$1200.000000 \quad 60.001732$

$2400.000000 \quad 60.115795$

$4800.000000 \quad 60.172989$

/SOURCE

60LEGV -1

9999

/ SWITCH

NODLAF LEGV

\$EOF User-supplied header cards follow.

ARG, NODLAF, NODLAT

\section{Appendix A.12: RSA.PCH}

\section{Frequency-dependent resistance model for phase-A series winding}

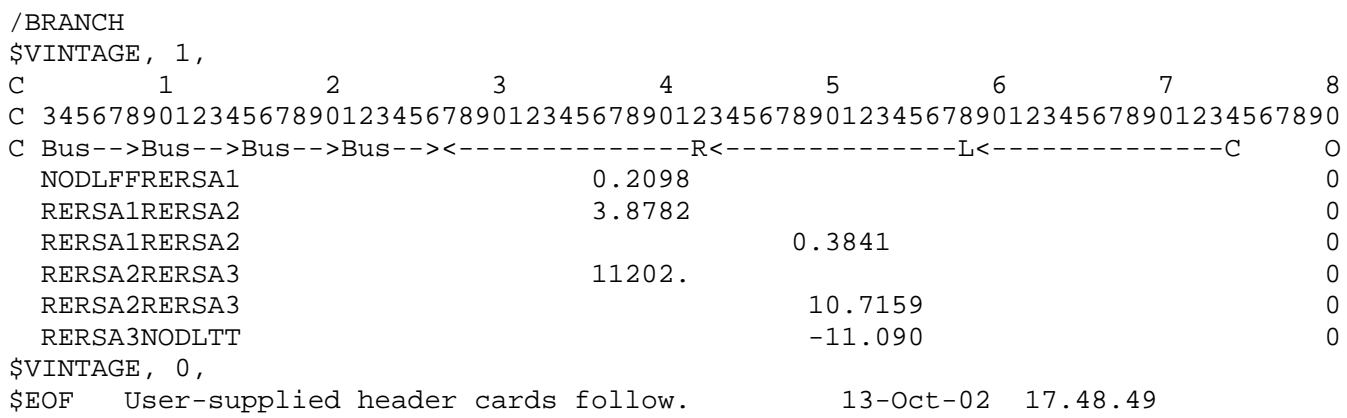

ARG, NODLFF, NODLTT

\section{Appendix A.13: RSB.PCH}

Frequency-dependent resistance model for phase-B series winding

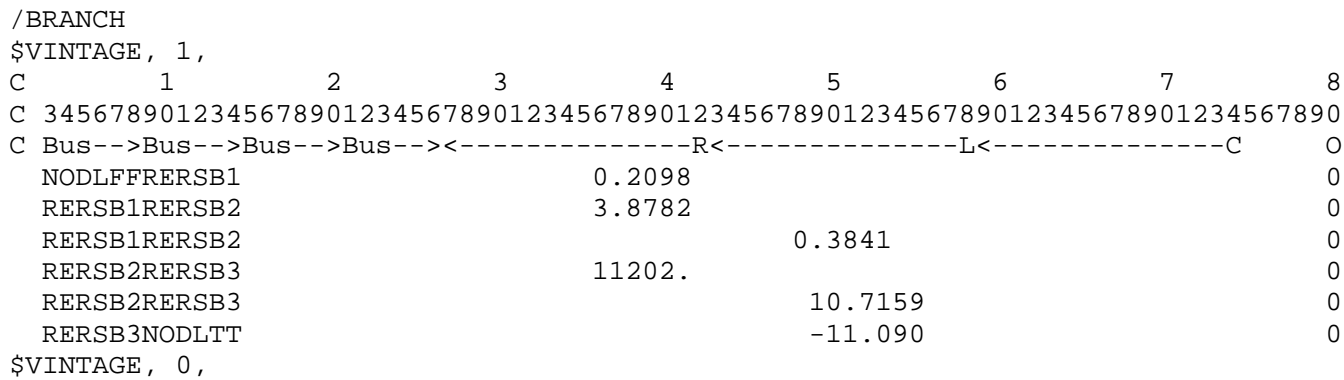


\$EOF User-supplied header cards follow.

ARG, NODLFF, NODLTT

\section{Appendix A.14: RSC.PCH}

Frequency-dependent resistance model for phase-C series winding

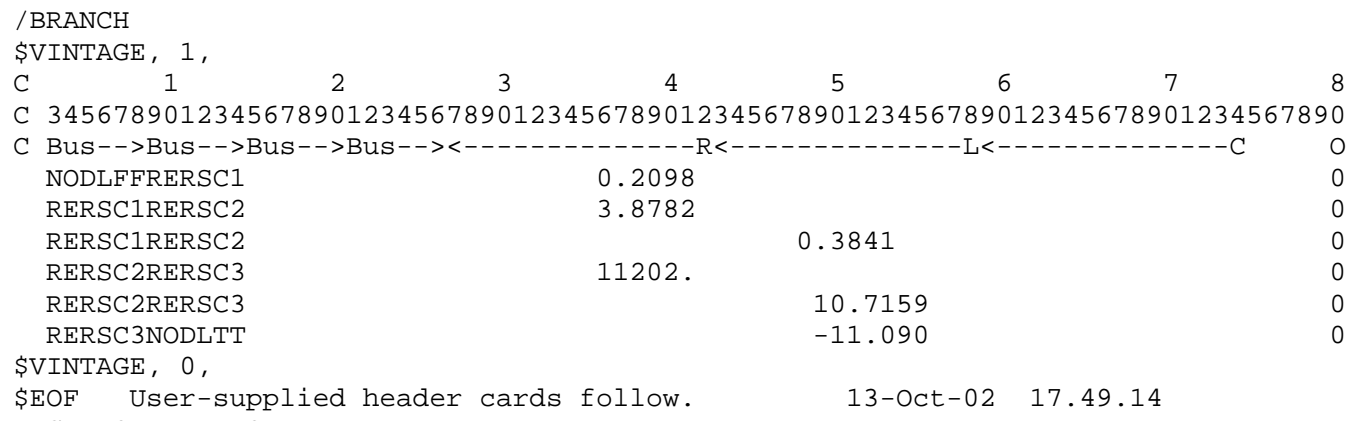

ARG, NODLFF, NODLTT

\section{Appendix A.15: RCA.PCH}

Frequency-dependent resistance model for phase-A common winding

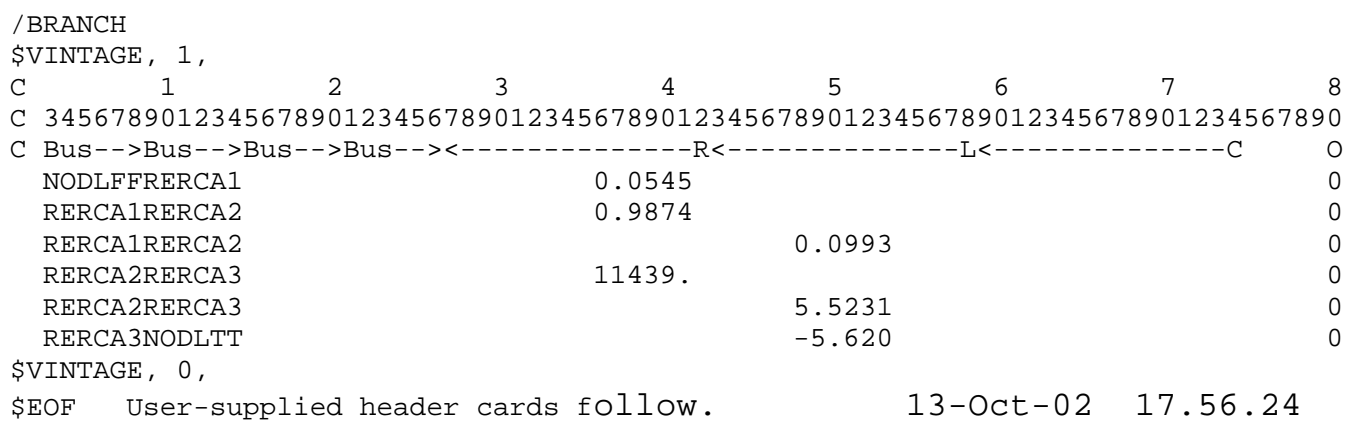

\section{Appendix A.16: RCB.PCH}

Frequency-dependent resistance model for phase-B common winding

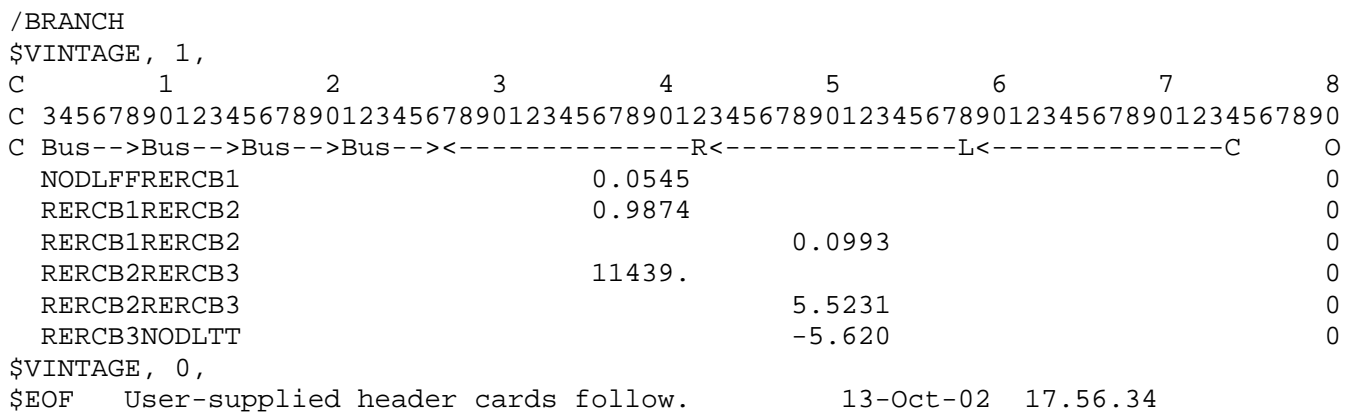

\section{Appendix A.17: RCC.PCH}

Frequency-dependent resistance model for phase-C common winding

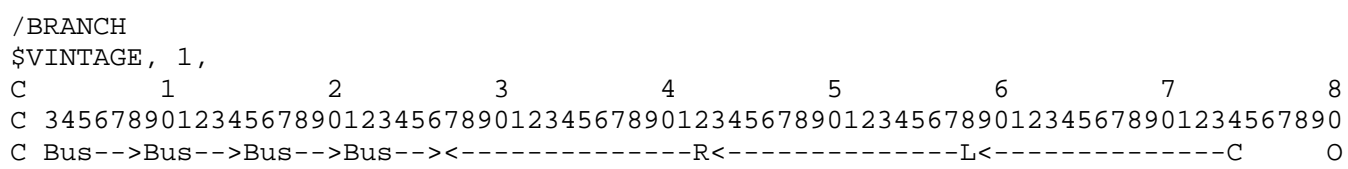




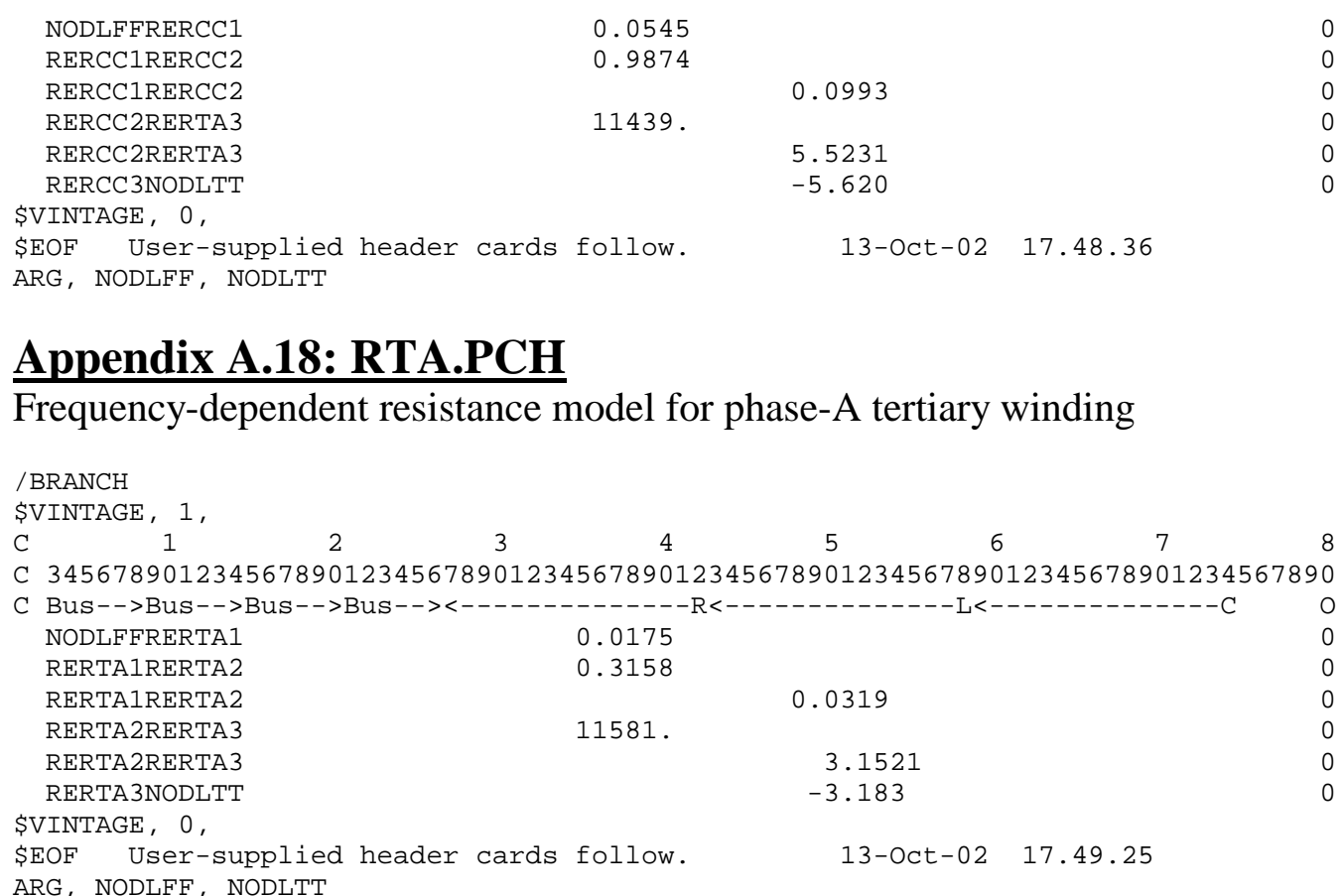

\section{Appendix A.19: RTB.PCH}

Frequency-dependent resistance model for phase-B tertiary winding

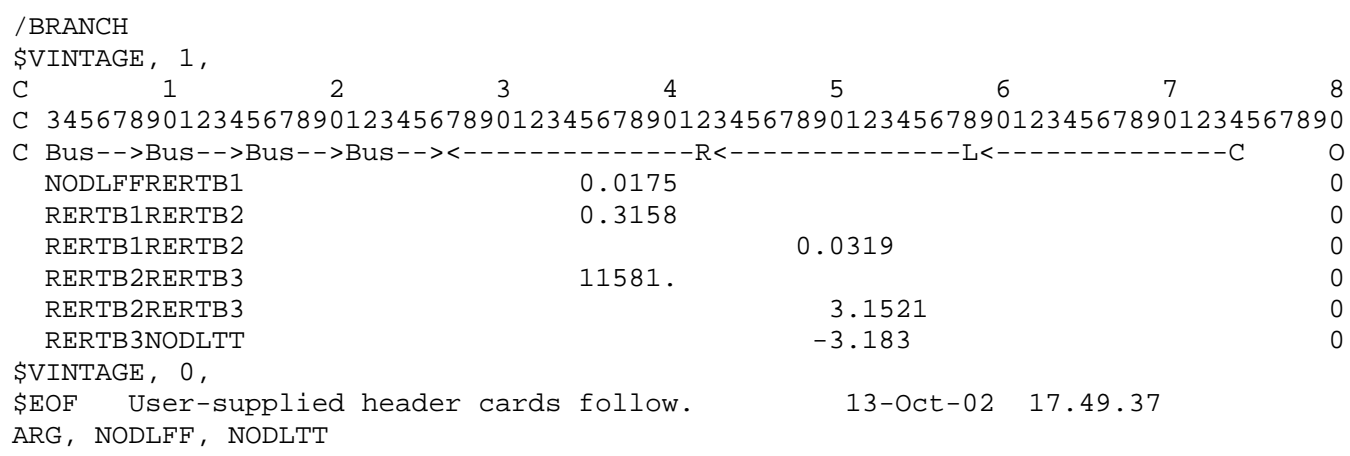

\section{Appendix A.20: RTC.PCH}

Frequency-dependent resistance model for phase-C tertiary winding

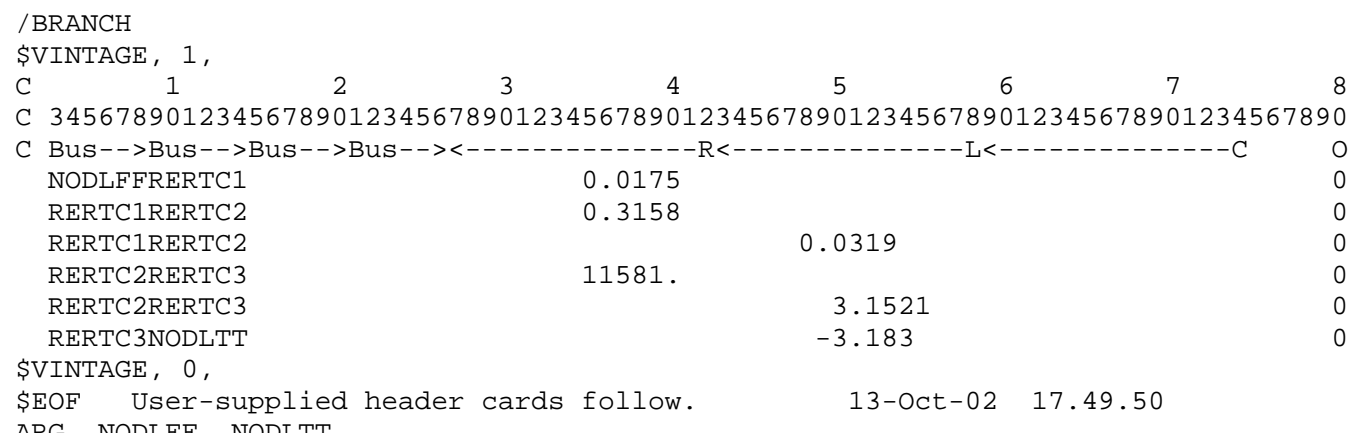

ARG, NODLFF, NODLTT 


\title{
APPENDIX B: MATLAB CODE LISTING
}

\author{
Appendix B.1: TRA5C5D1.m \\ Parameter Estimation of B-H curve for Five-Legged Core Transformer \\ warning off \\ clear \\ clc \\ format compact; format short \\ $\mathrm{A}(1)=1 . ; \mathrm{A}(2)=1 . ; \mathrm{A}(3)=1 . ; \mathrm{A}(4)=1 ; \mathrm{A}(5)=1 ; \mathrm{A}(6)=1 ; \mathrm{A}(7)=1$;

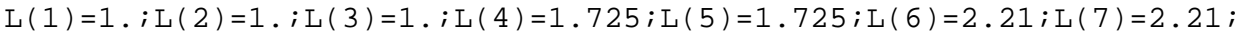 \\ $\therefore$ peaki=76.17 and 278.90 \\ irms $=\left[\begin{array}{ll}105.020 & 224.112\end{array}\right]$ \\ $\mathrm{x} 0=[4.2 .42] ; \quad \%$ Starting Guess \\ ooptions=optimset ('LargeScale', 'off', 'MaxIter', 1000) \\ options. LargeScale= 'off' \\ options=optimset ('Display','iter'); \% Option to display output \\ $\mathrm{AA}=[] ; \mathrm{bb}=[] ; \mathrm{Aeq}=[] ; \mathrm{beq}=[] ;$ \\ $1 \mathrm{~b}=[.5 .01] ; \mathrm{ub}=\left[\begin{array}{ll}20 & .6\end{array}\right] ;$ \\ $[\mathrm{x}$, fval, exitflag, output $]=$ fmincon ('TRA5C5D1fun' , x0, AA, bb, Aeq, beq, lb, ub) \\ $\mathrm{a}=\mathrm{x}(1)$ \\ $\mathrm{b}=\mathrm{x}(2)$ \\ $\mathrm{N}=34$ \\ $\mathrm{V}=1.0 ; \mathrm{RMSi}=0 ; \mathrm{RMSlam}=0$; init $=0$; \\ for ang=0:pi/40:pi*1-pi/40 \\ $\mathrm{x} 0=[$ init ang $\mathrm{a} \mathrm{b} \mathrm{V}]$; $\frac{\circ}{\circ}$ Starting Guessoptions.LargeScale='off'; \\ options=optimset ('Display','iter'); $\%$ Option to display output \\ $\mathrm{AA}=[] ; \mathrm{b} \mathrm{b}=[] ; \mathrm{Aeq}=[] ; \mathrm{beq}=[] ;$ \\ $l b=[-55$ ang $a b$ b $] ; u b=[55$ ang a b V]; \\ $\%[x$, fval, exitflag, output $]=$ \\ fmincon ('TRA5C5F fun' ' x0, AA, bb, Aeq, beq, 1b, ub, 'TRA5C5Ftestcon' ) \\ $[y, f v a l]=$ fmincon ('TRA5C5F1fun', x0, AA, bb, Aeq, beq, 1b, ub) \\ init=y (1) ; \\ lamdax=y (1); \\ lamda $(1)=\sin ($ ang $) * 51.77 * V$; \\ lamda $(2)=\sin ($ ang $-\mathrm{pi} * 2 / 3) \star 51.77 * \mathrm{~V}$; \\ lamda $(3)=\sin ($ ang $+\mathrm{pi} * 2 / 3) * 51.77 * \mathrm{~V}$; \\ lamda ( 4$)=-($ lamda (1) /3-lamda (2) /3-lamdax); \\ lamda (5) $=-($ lamda (2) /3-lamda (3)/3-lamdax $)$; \\ lamda (6) $=-($ lamda (3) /3-lamda (1)/3-lamdax) ; \\ lamda $(7)=$ lamda (6) ; \\ $\mathrm{B}(1)=$ lamda $(1) / \mathrm{N} / \mathrm{A}(1)$; \\ $\mathrm{B}(2)=\mathrm{lamda}(2) / \mathrm{N} / \mathrm{A}(2)$; \\ $\mathrm{B}(3)=$ lamda $(3) / \mathrm{N} / \mathrm{A}(3)$; \\ $\mathrm{B}(4)=1$ amda $(4) / \mathrm{N} / \mathrm{A}(4)$; \\ $\mathrm{B}(5)=$ lamda $(5) / \mathrm{N} / \mathrm{A}(5)$; \\ $\mathrm{B}(6)=\mathrm{lamda}(6) / \mathrm{N} / \mathrm{A}(6)$; \\ $\mathrm{B}(7)=\mathrm{B}(6)$; \\ $i={ }^{\star}{ }^{a} \cdot{ }^{*} \mathrm{~B} \cdot /\left(1-\mathrm{abs}(\mathrm{B}) \cdot{ }^{\star} \mathrm{b}\right)$; \\ $\operatorname{ABSi}(1)=i(1)+i(6)+i(7)-i(3)$; \\ $\operatorname{ABSi}(2)=i(2)+i(4)-i(1)$; \\ $\operatorname{ABSi}(3)=i(3)+i(5)-i(2)$; \\ RMSi=RMSi+ABSi.^2; \\ RMSlam=RMSlam+lamda (1)^2;
}




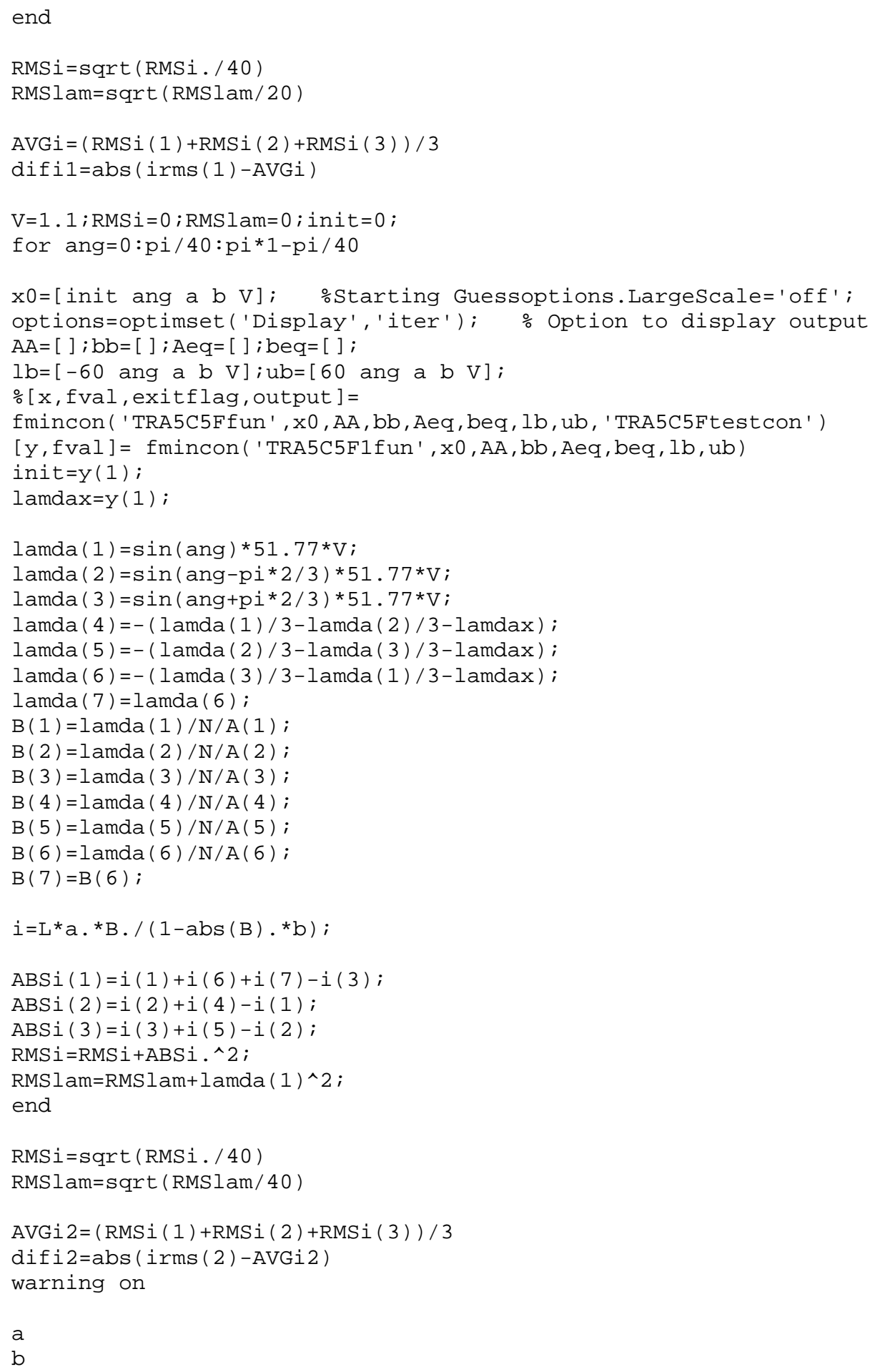

\section{Appendix B.2: TRA5S1fun.m}

Parameter estimation of B-H curve for Five-Legged Core Transformer

function $F=$ TRA5C5D1fun (x) ;

$\mathrm{A}(1)=1 . ; \mathrm{A}(2)=1 . ; \mathrm{A}(3)=1 . ; \mathrm{A}(4)=1 ; \mathrm{A}(5)=1 ; \mathrm{A}(6)=1 ; \mathrm{A}(7)=1$; 


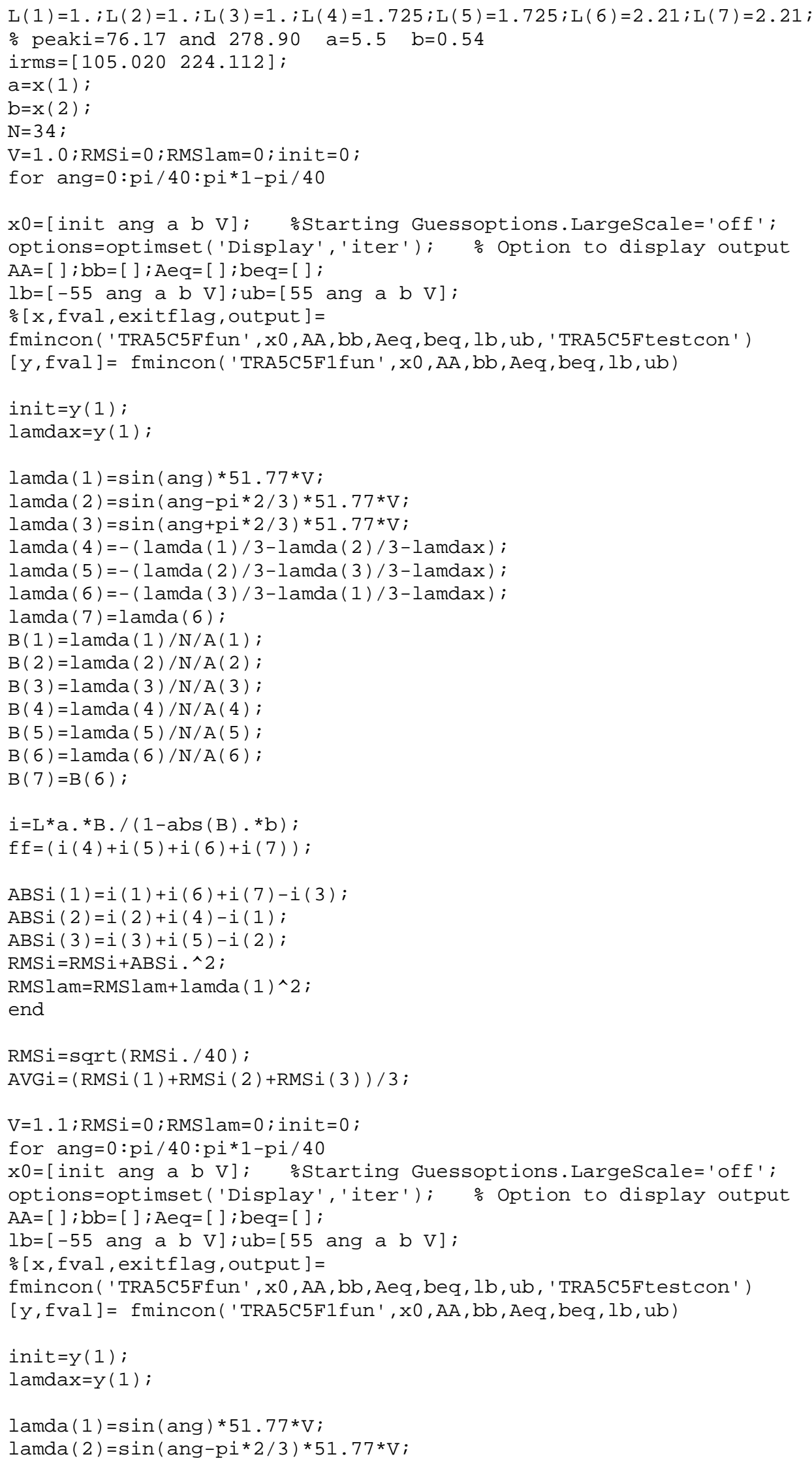




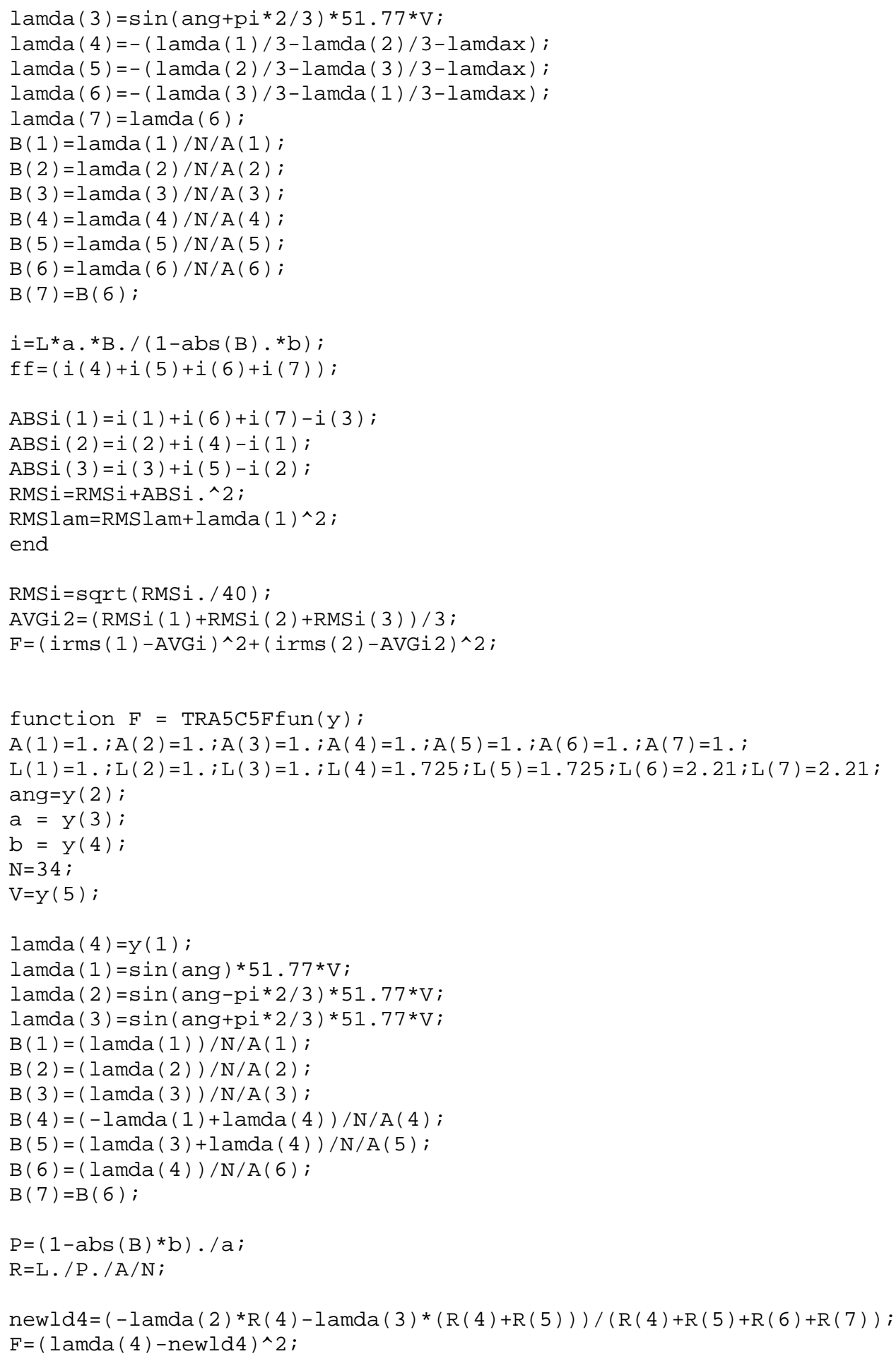

\section{Appendix B.3: LTRC5.m}

Parameter estimation of Core loss curve for Five-Legged Core Transformer

clear

clc

format compact; format short 


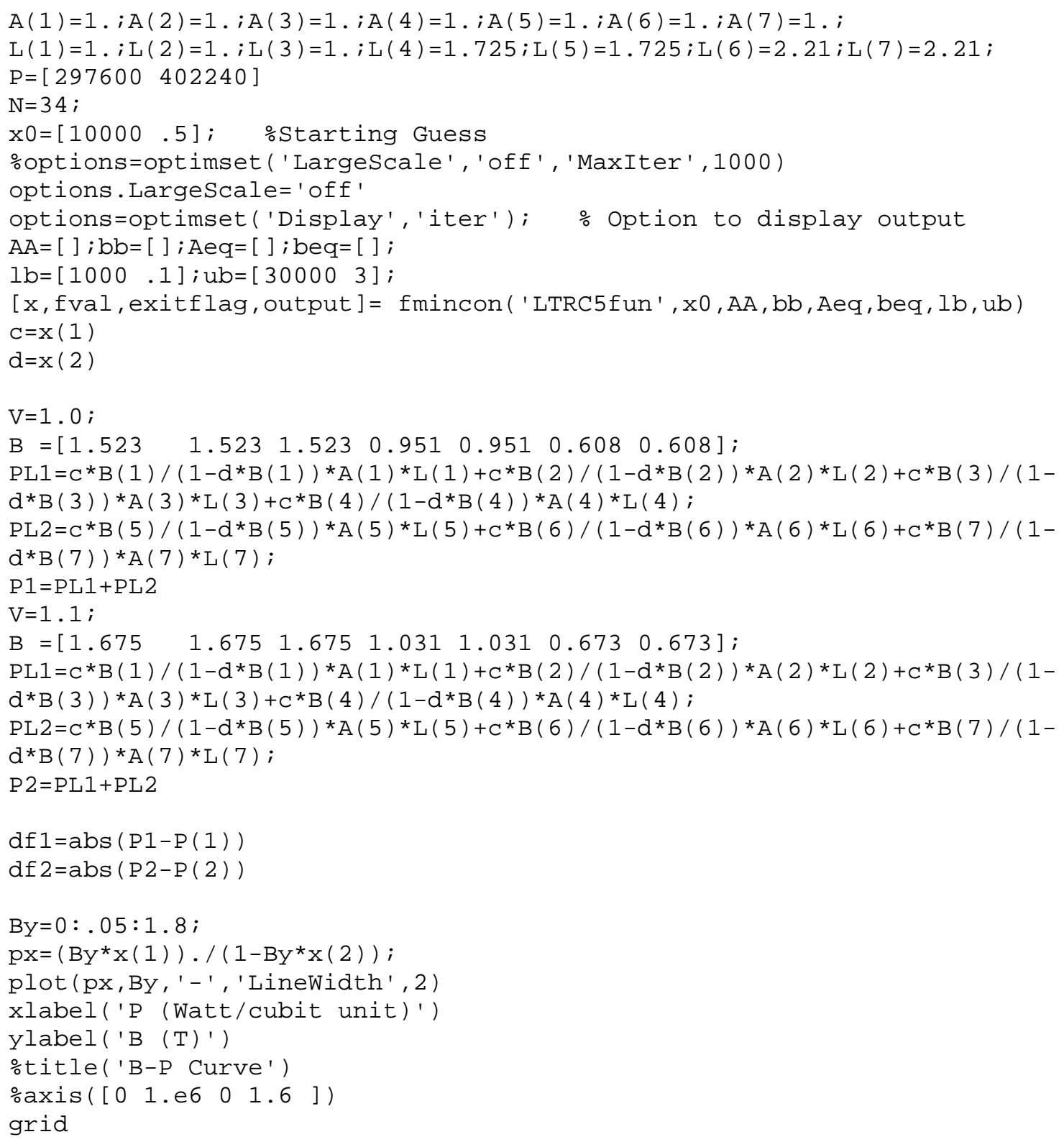

\section{Appendix B.4: LTRC5fun.m}

\section{Parameter estimation of core loss curve for Five-Legged Core Transformer}

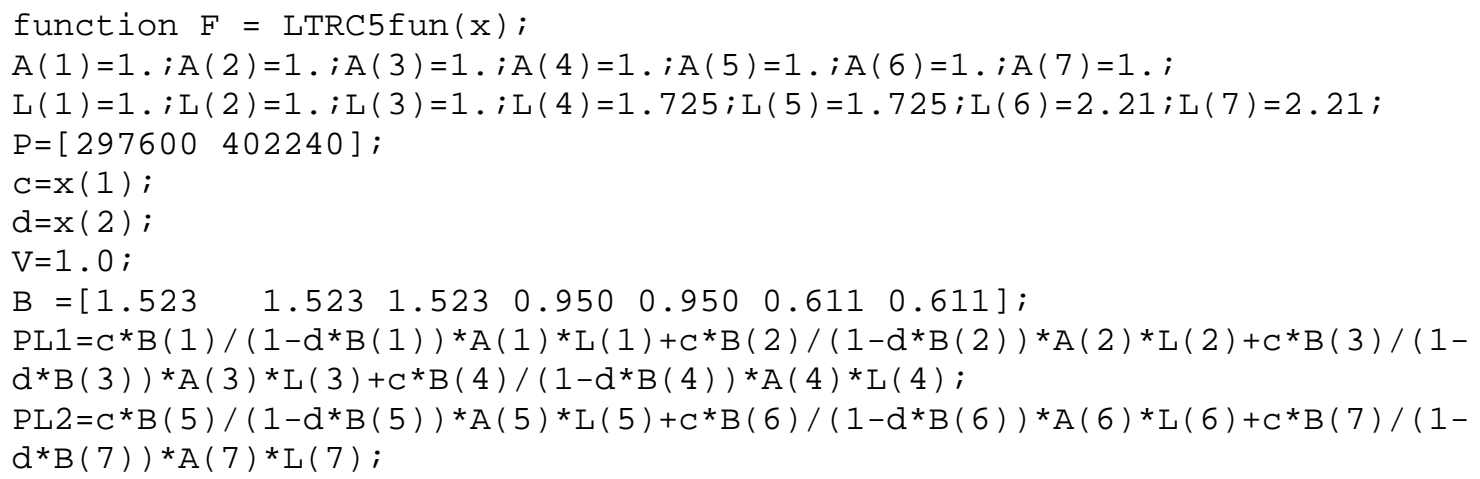




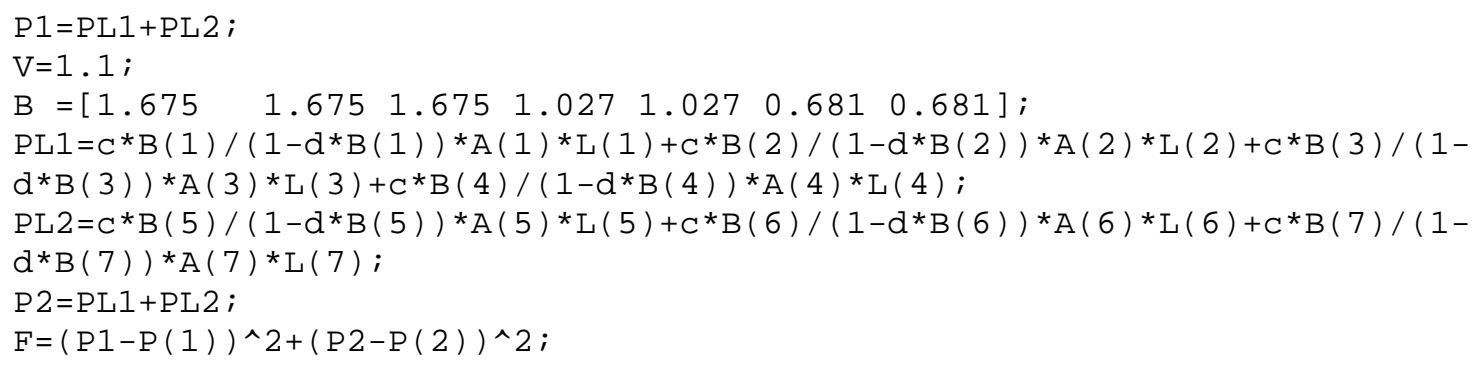

\section{Appendix B.5: AHC5.m}

Parameter estimation of Hysteresis Loss curve for Five-Legged Core Transformer

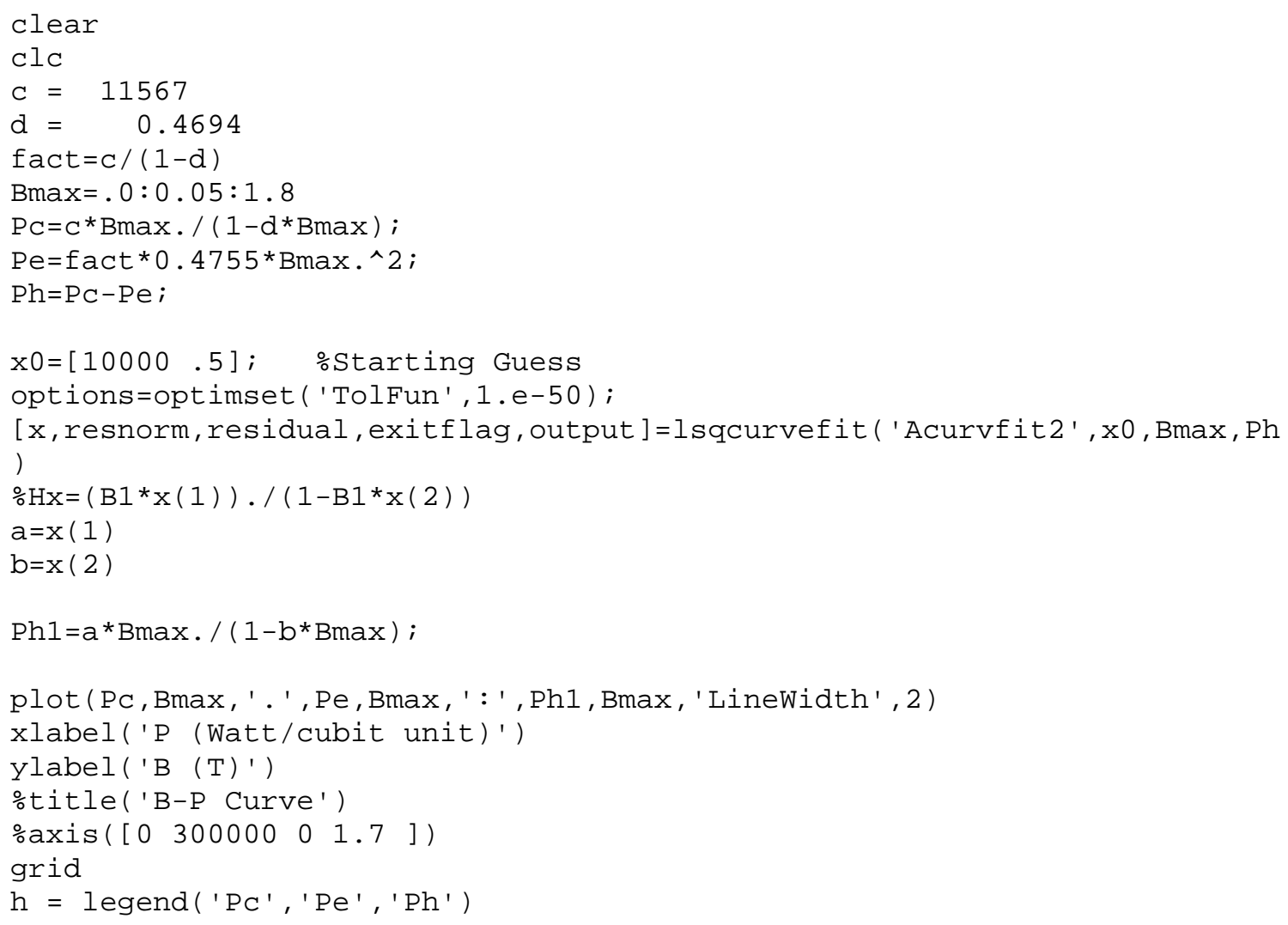

\section{Appendix B.6: Acurvfit2.m}

Parameter estimation of hysteresis loss curve for Five-Legged Core Transformer

function $F=$ curvfit $2(x, B)$;

$\mathrm{F}=(\mathrm{B} \star \mathrm{X}(1)) \cdot /\left(1-\mathrm{B}^{\star} \mathrm{X}(2)\right)$;

\section{Appendix B.7: AhC5A.m}

Estimation of maximum coercive force for Five-Legged Core Transformer

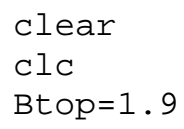




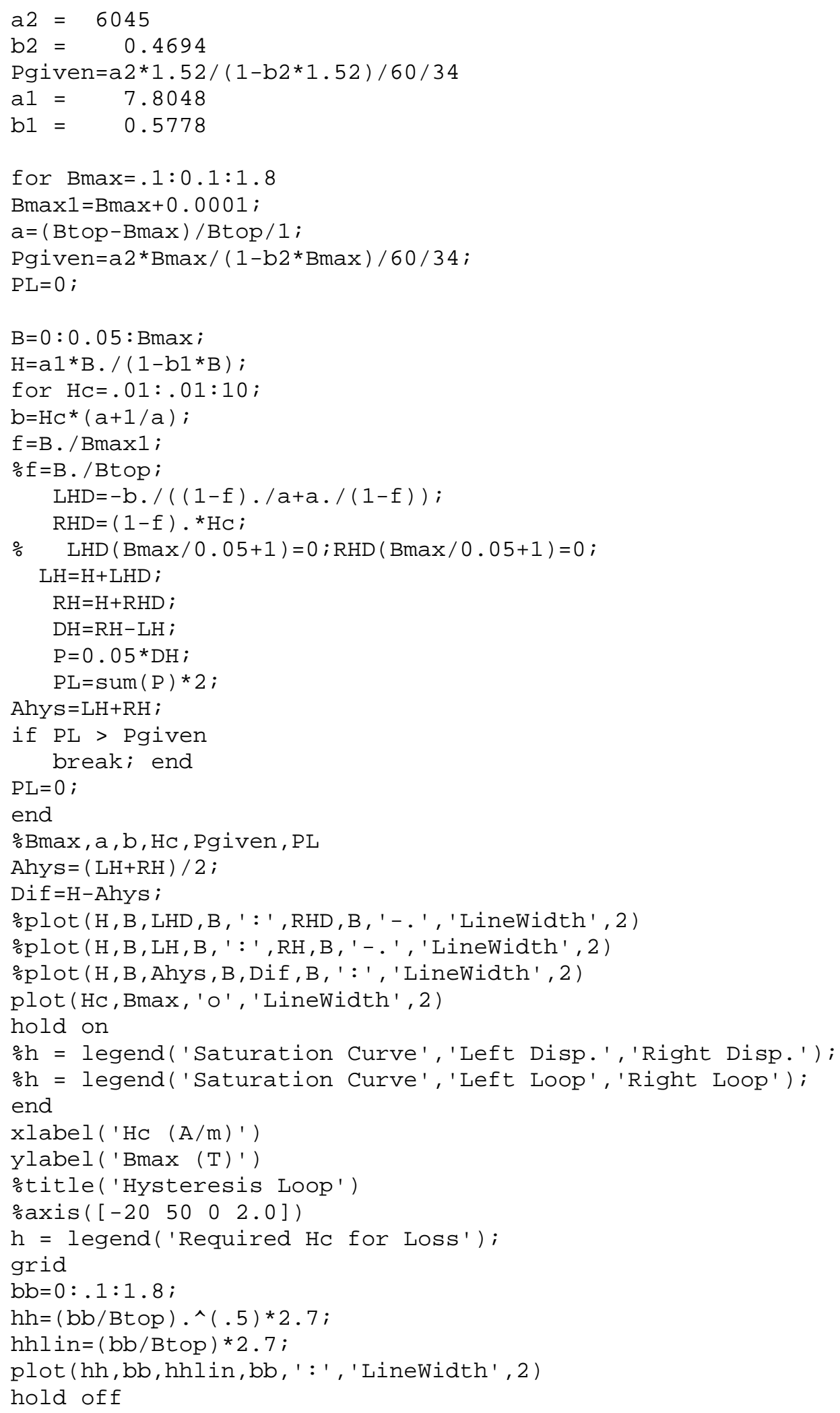




\section{Appendix B.8: TRA5C3A1.m}

\section{Parameter estimation of B-H curve for Three-Legged Core Transformer}

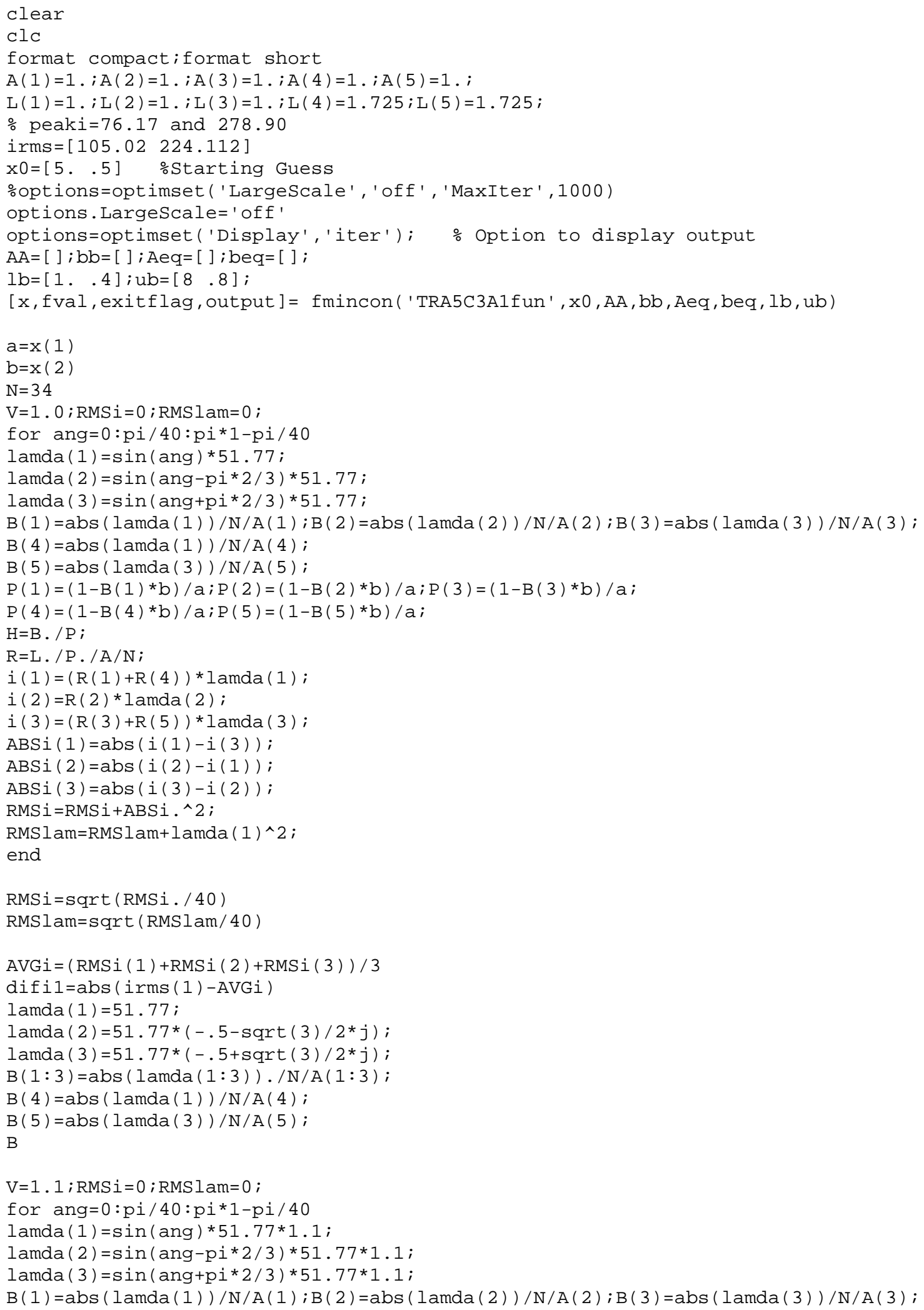




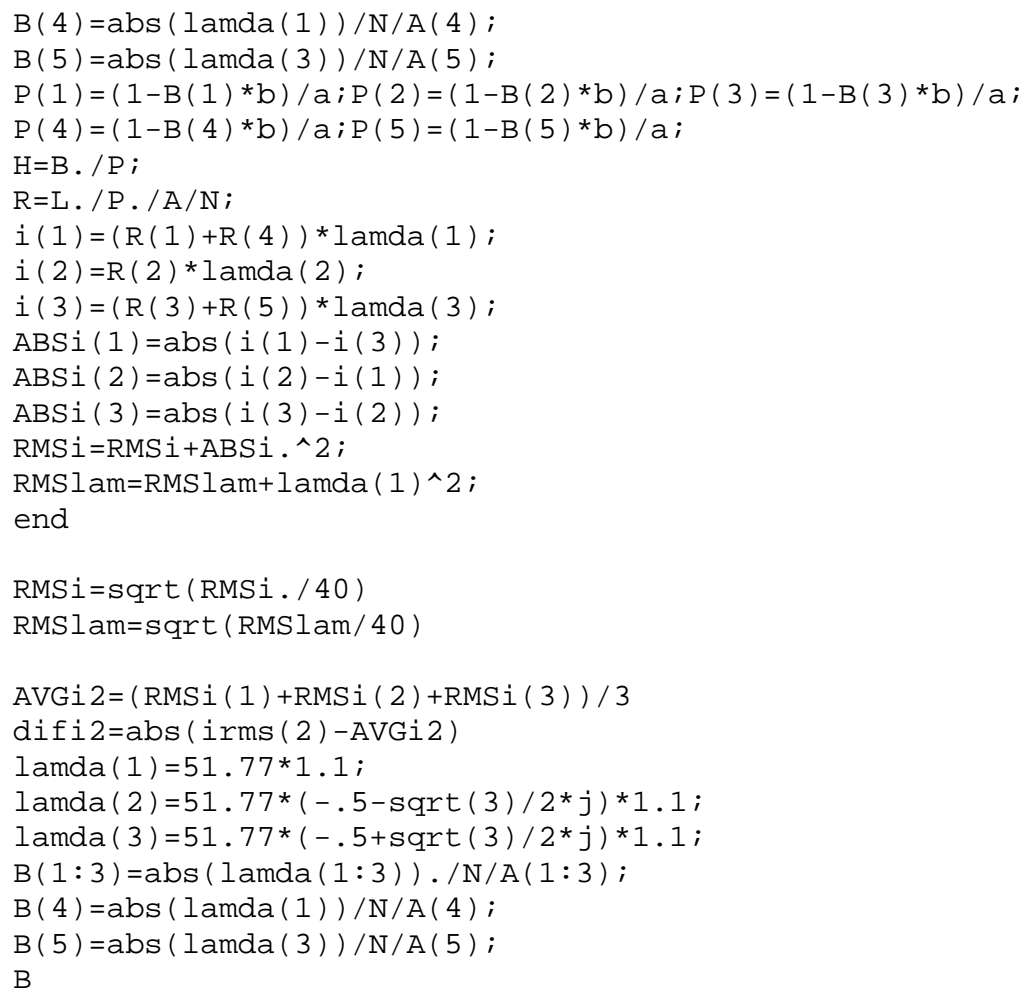

\section{Appendix B.9: TRA5C3A1fun.m}

\section{Parameter estimation of B-H curve for Three-Legged Core Transformer}

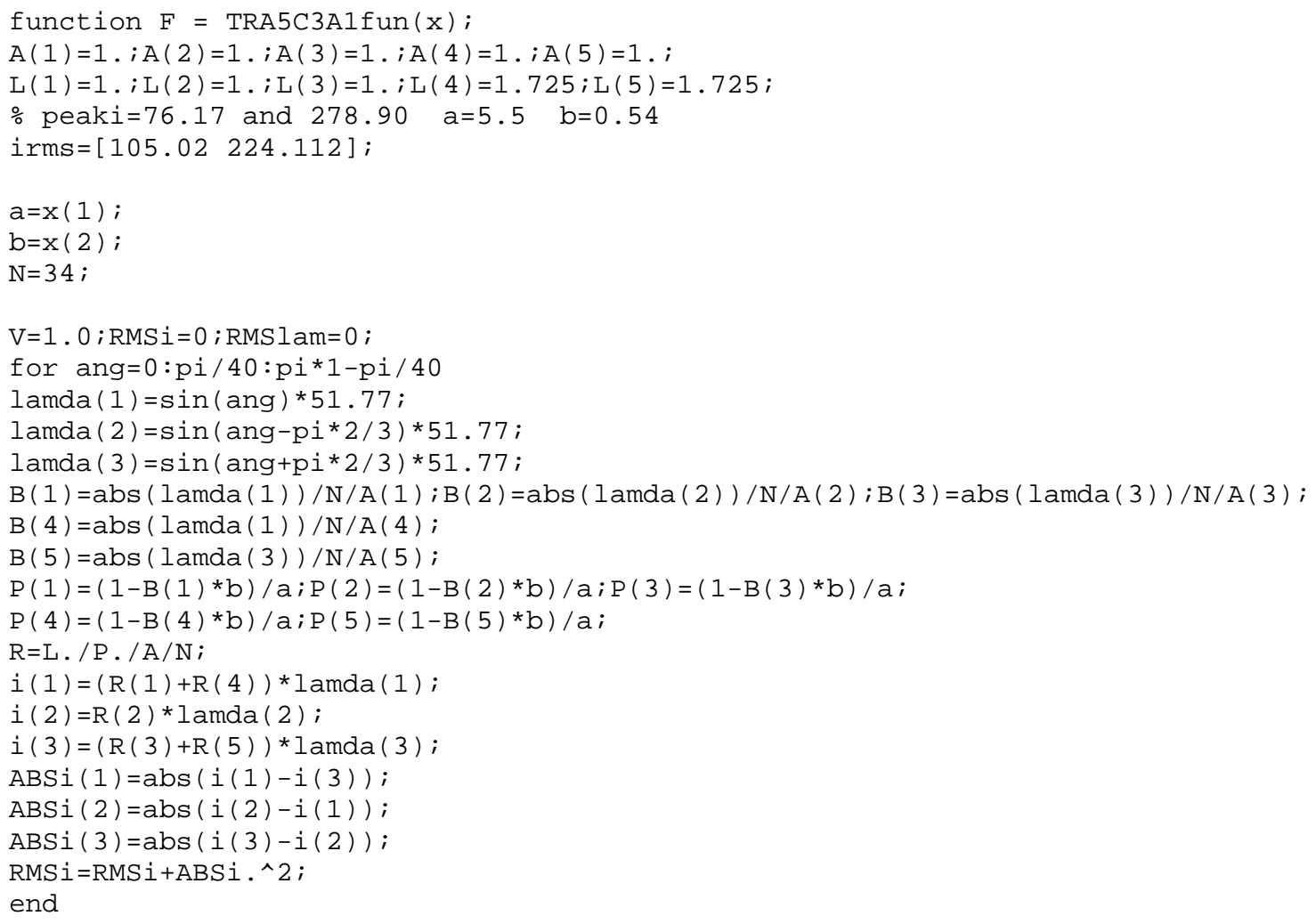




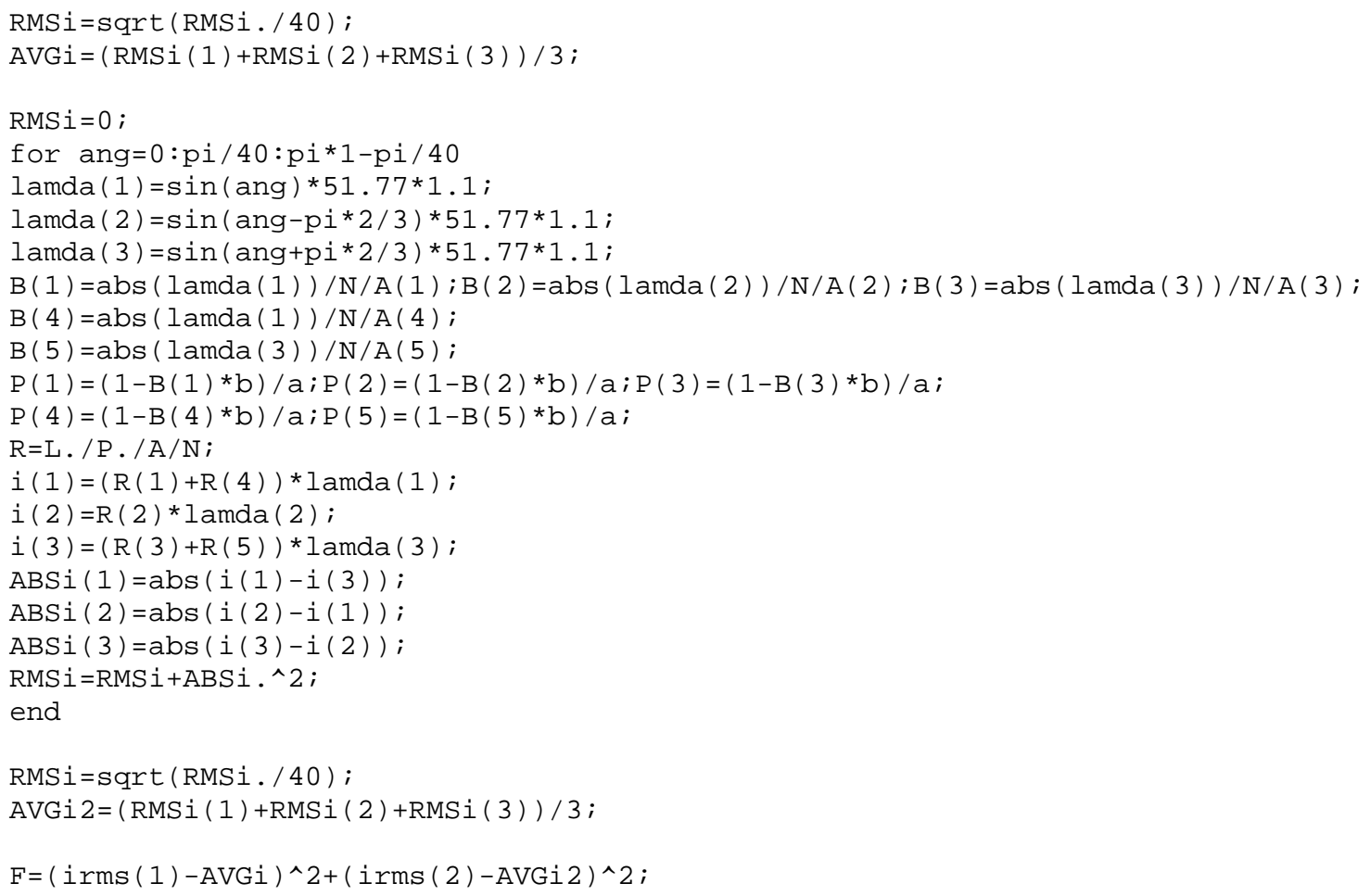

\section{Appendix B.10: LTC3.m}

\section{Parameter estimation of core loss curve for Three-Legged Core Transformer}

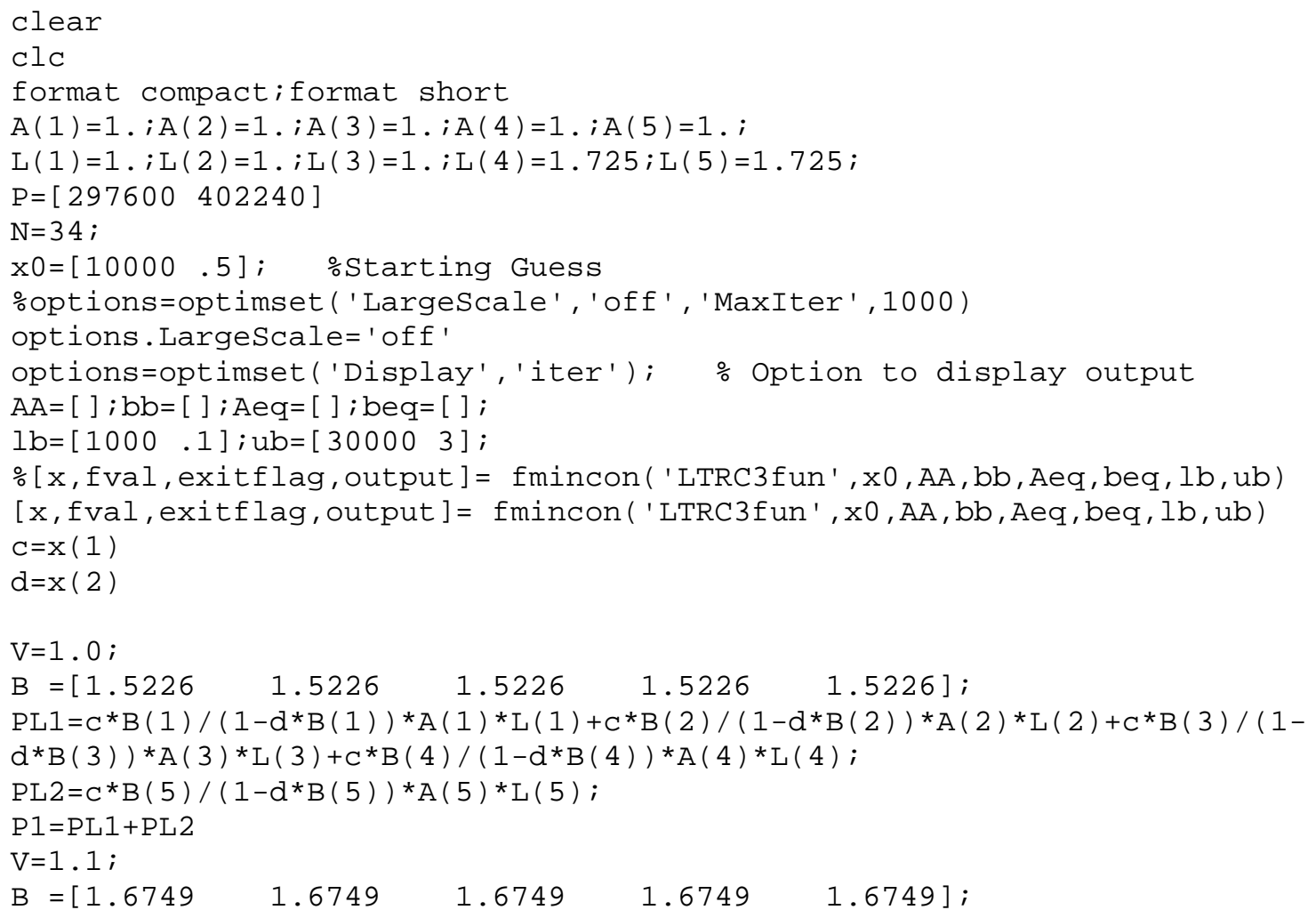




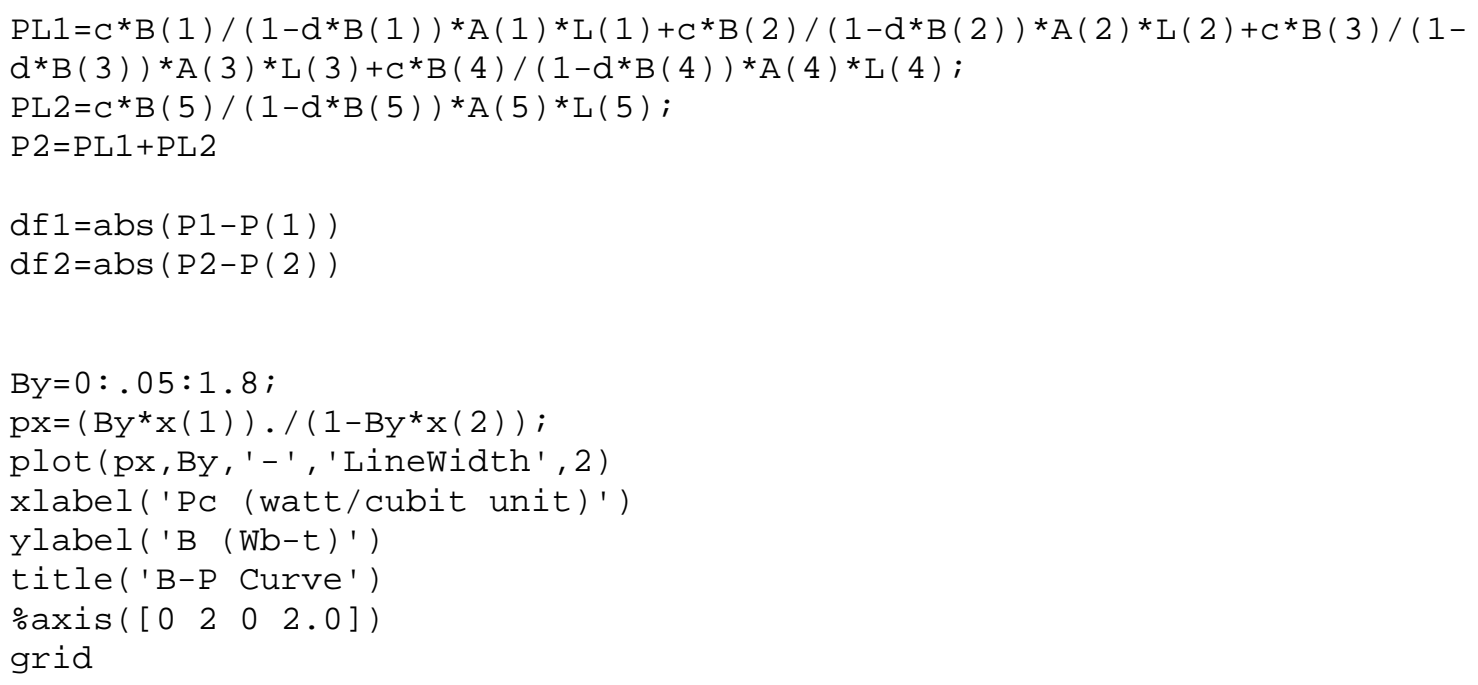

\section{Appendix B.11: LTC3fun.m}

Parameter estimation of core loss curve for Three-Legged Core Transformer

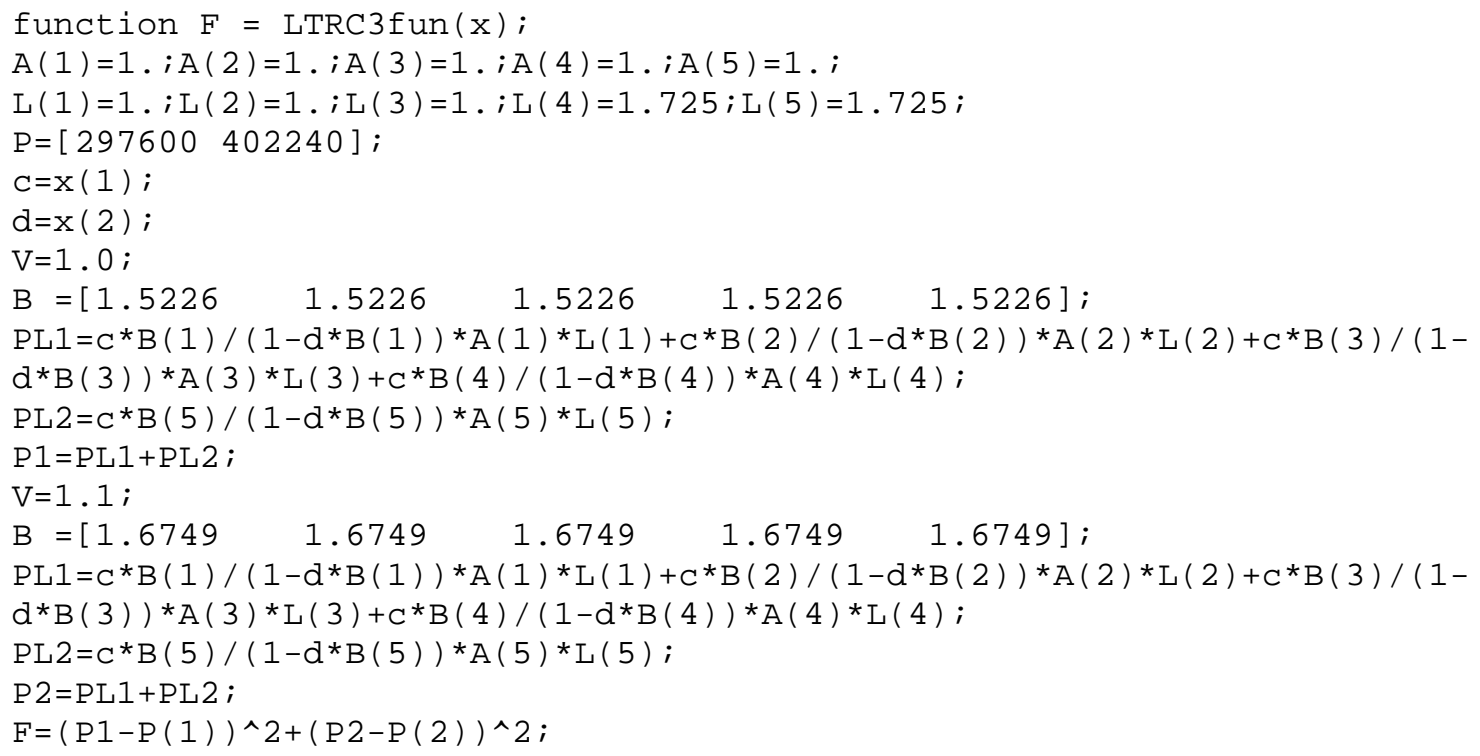

\section{Appendix B.12: AHC3.m}

Parameter estimation of Hysteresis Loss curve for Three-Legged Core Transformer

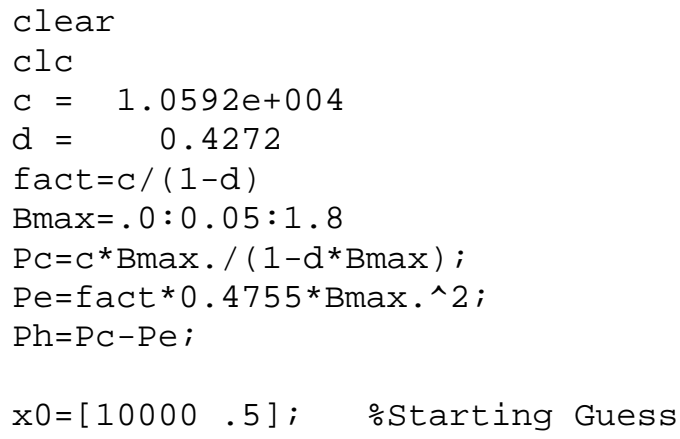




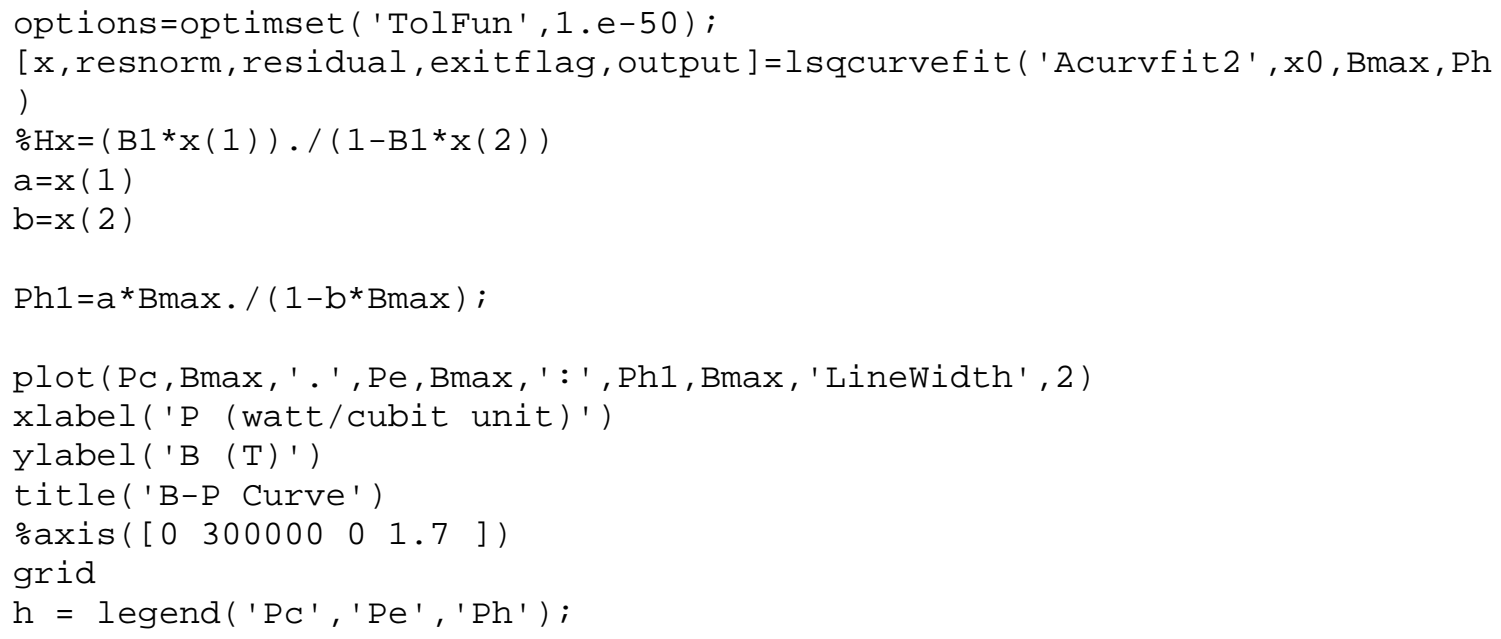

\section{Appendix B.13: Acurvfit2.m}

Parameter estimation of Hysteresis loss curve for Three-Legged Core Transformer

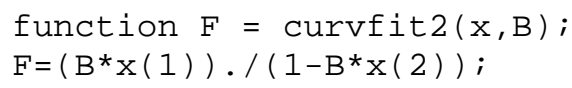

\section{Appendix B.14: AhC3A.m}

Estimation of Maximum coercive force for Three-Legged Core Transformer

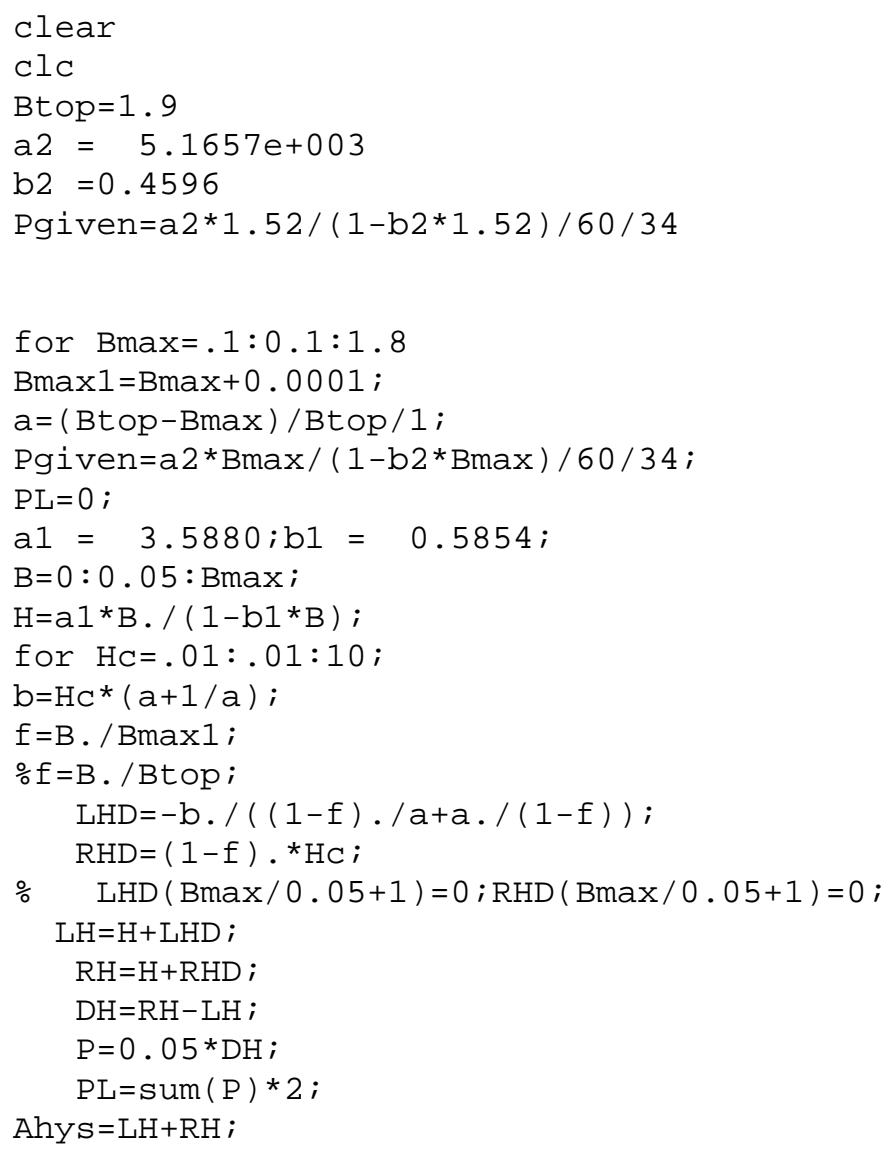




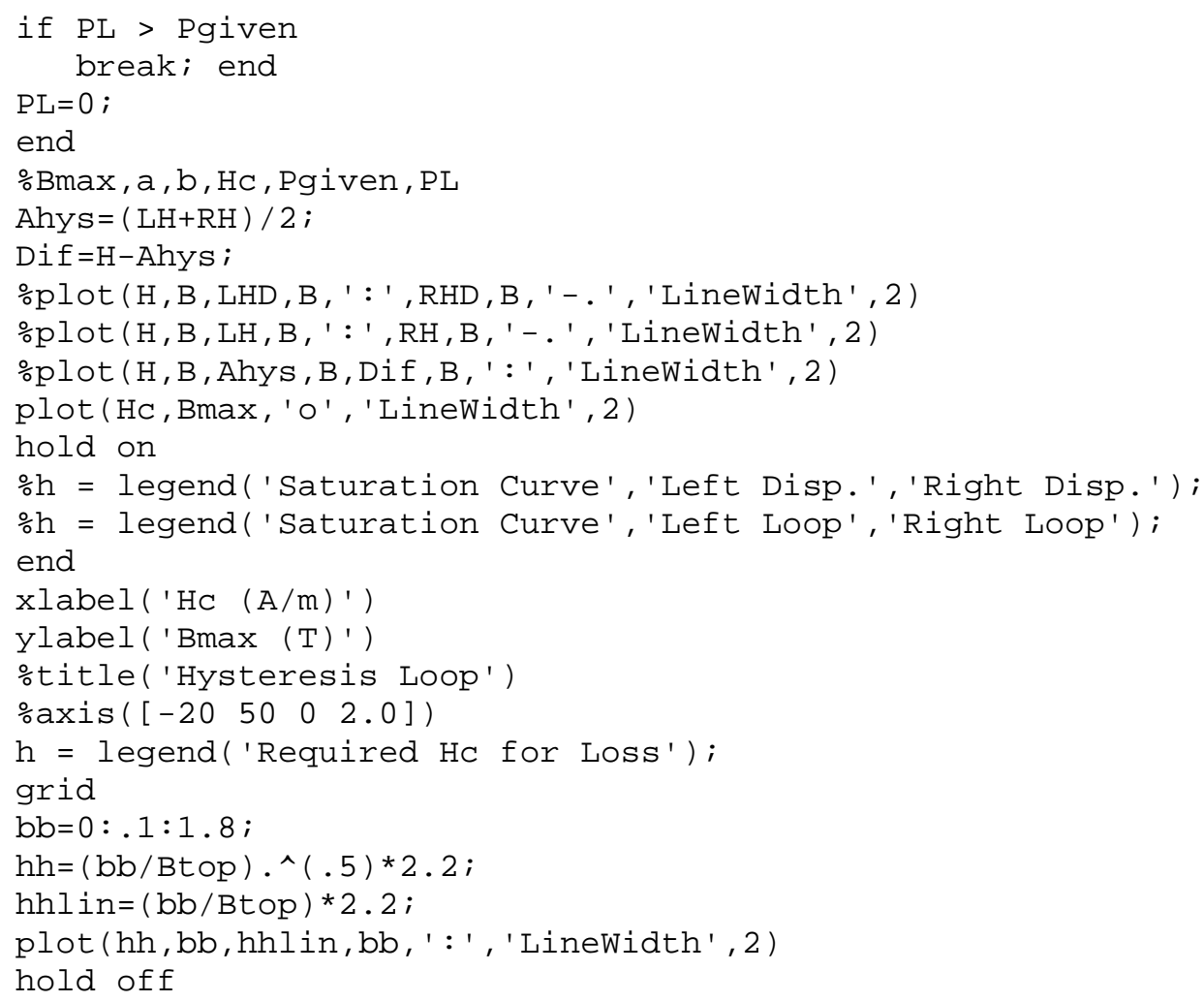




\section{Appendix B.15: TRA5S1.m}

\section{Parameter estimation of B-H curve for Shell-form transformer}

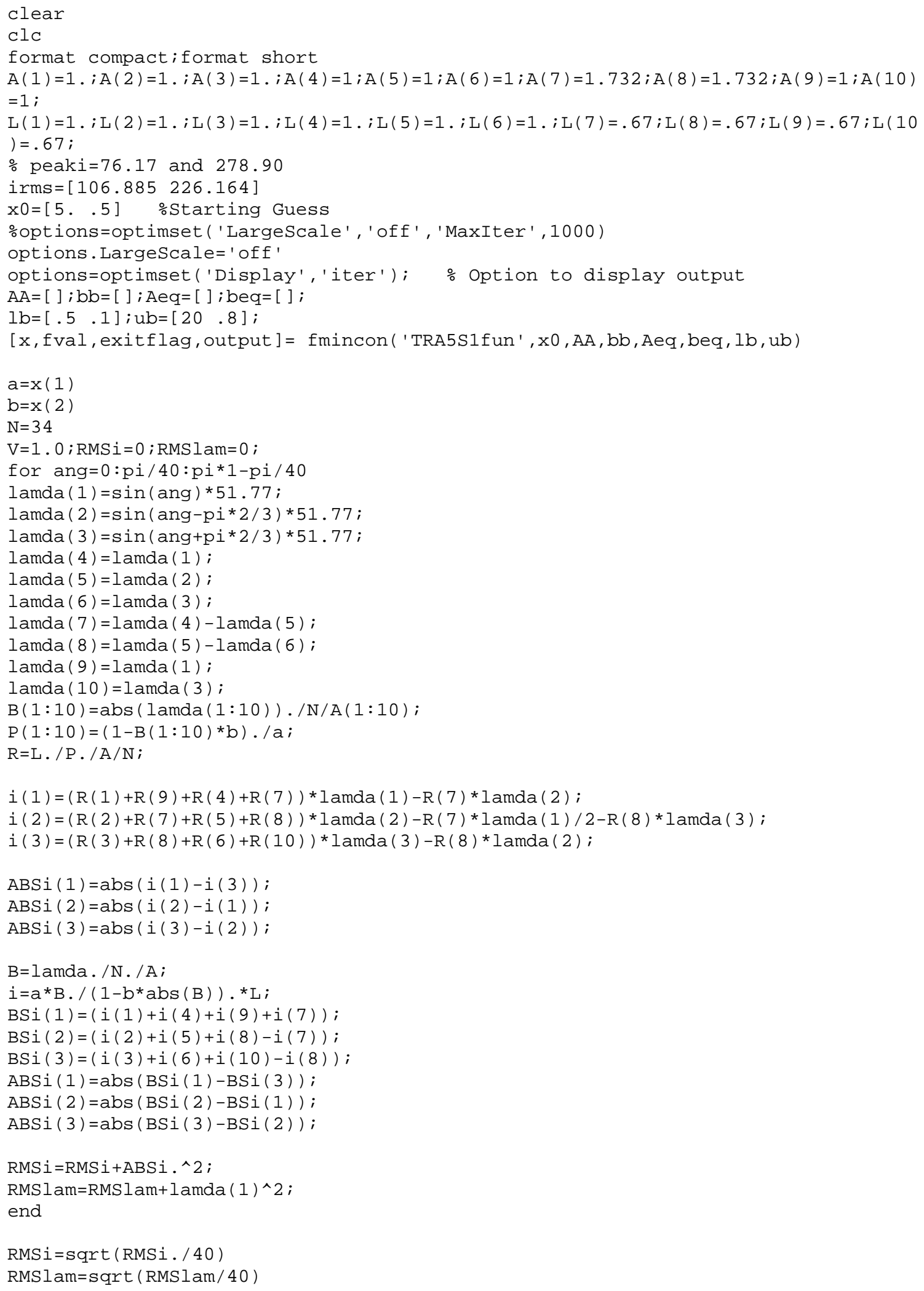




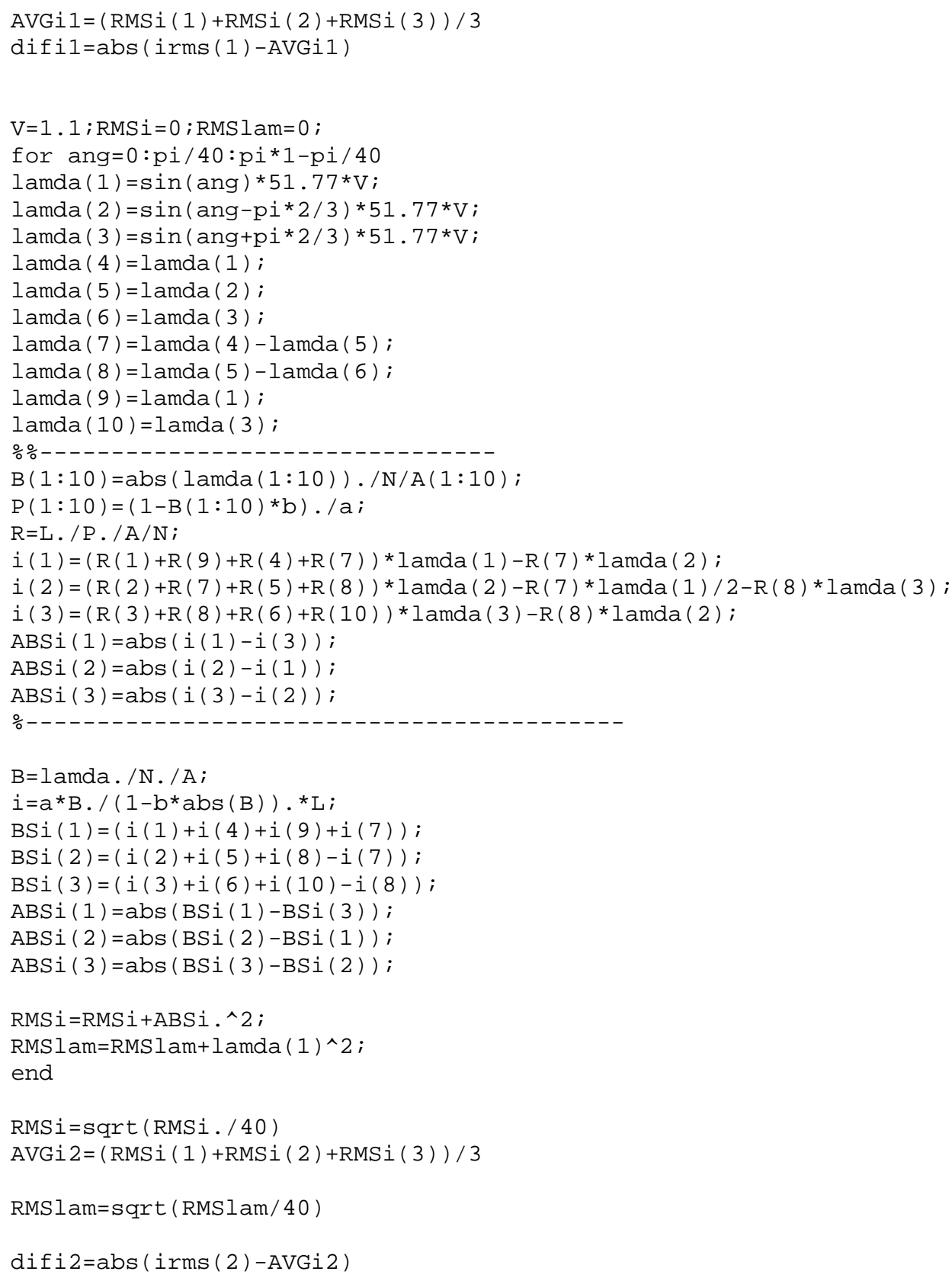

\section{Appendix B.16: TRA5S1fun.m}

\section{Parameter estimation of B-H curve for Shell-form transformer}

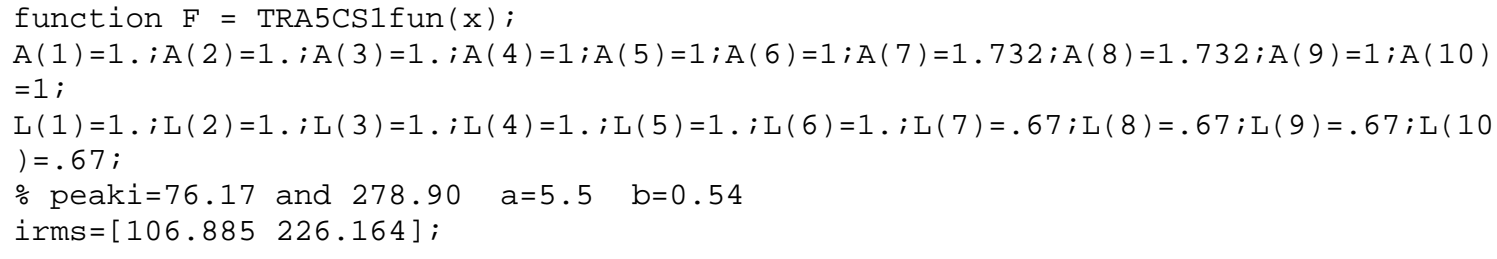




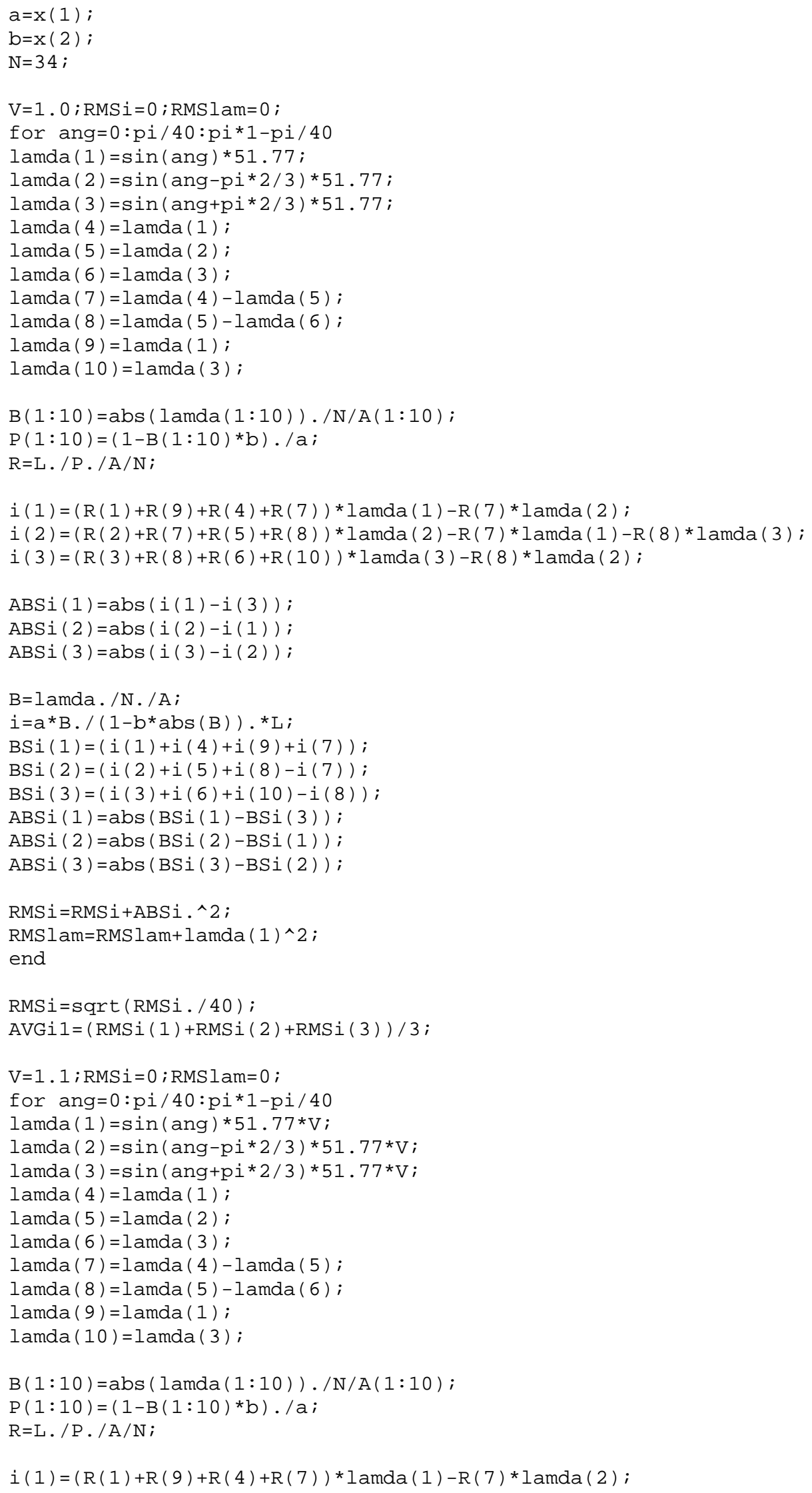




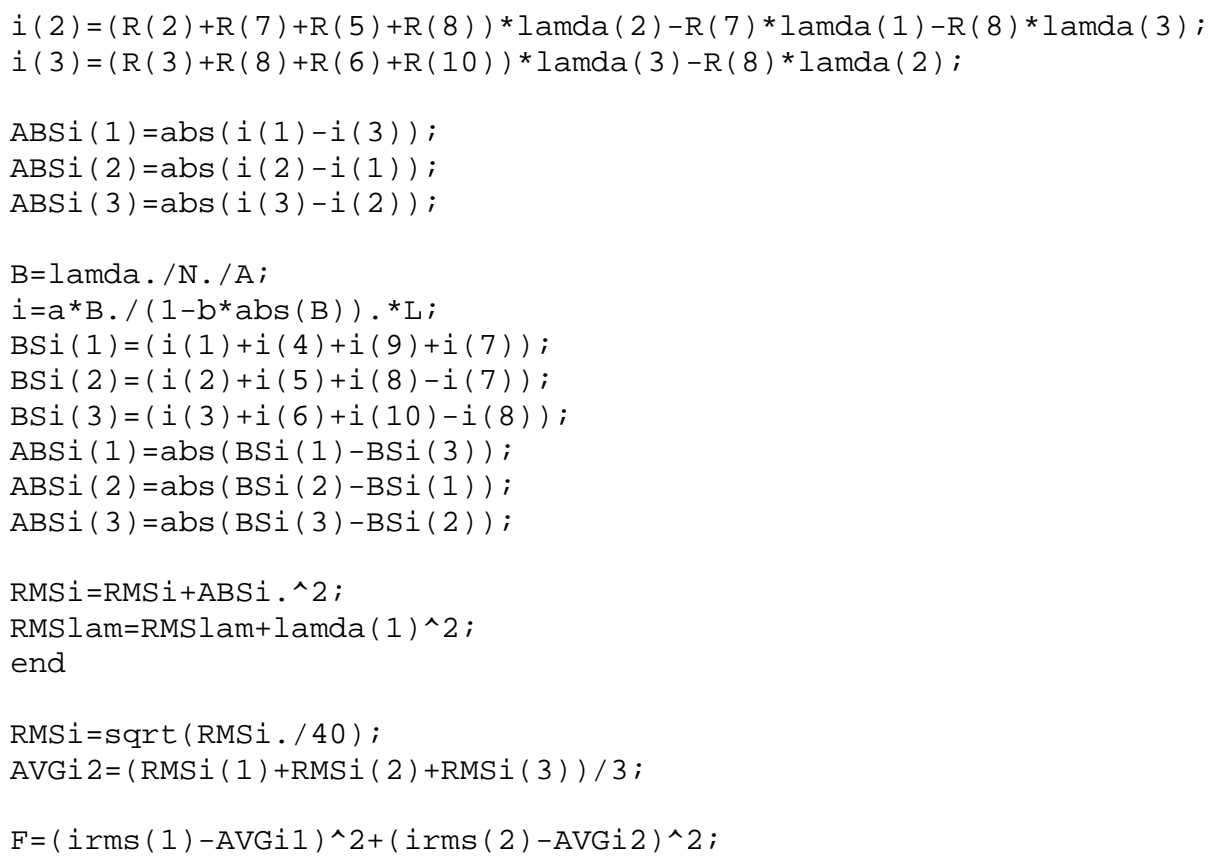

\section{Appendix B.17: LTS.m}

\section{Parameter estimation of core loss curve for shell-form transformer}

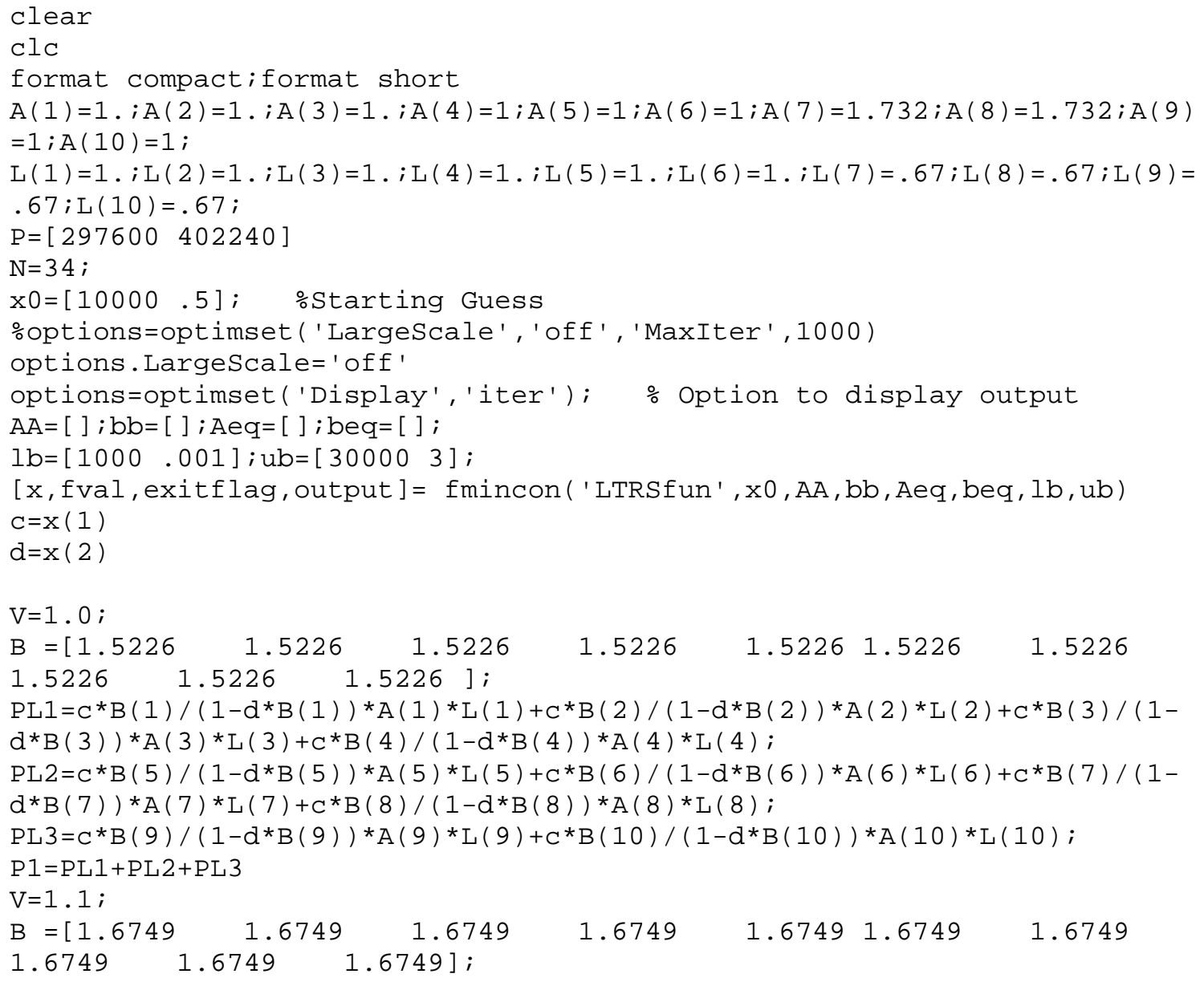




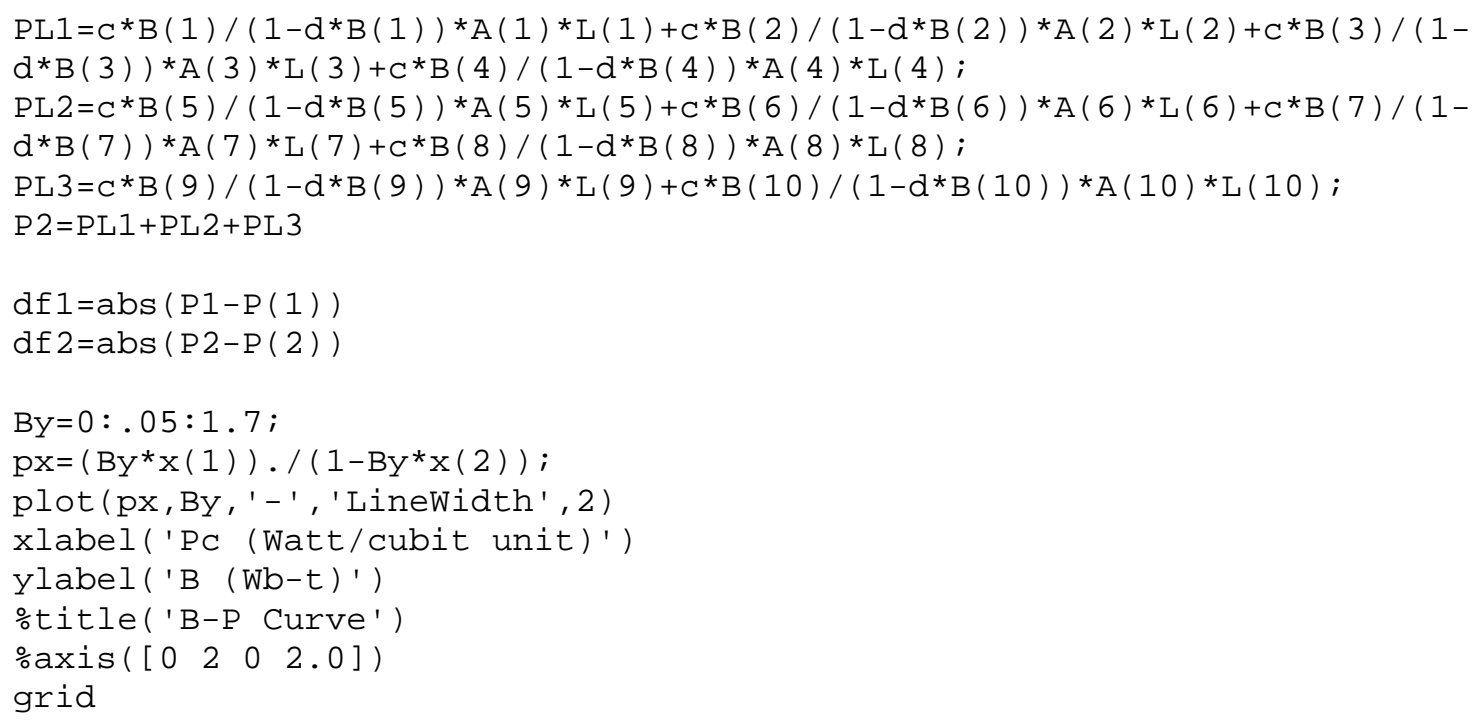

\section{Appendix B.18: LTSfun.m}

Parameter estimation of core loss curve for shell-form transformer

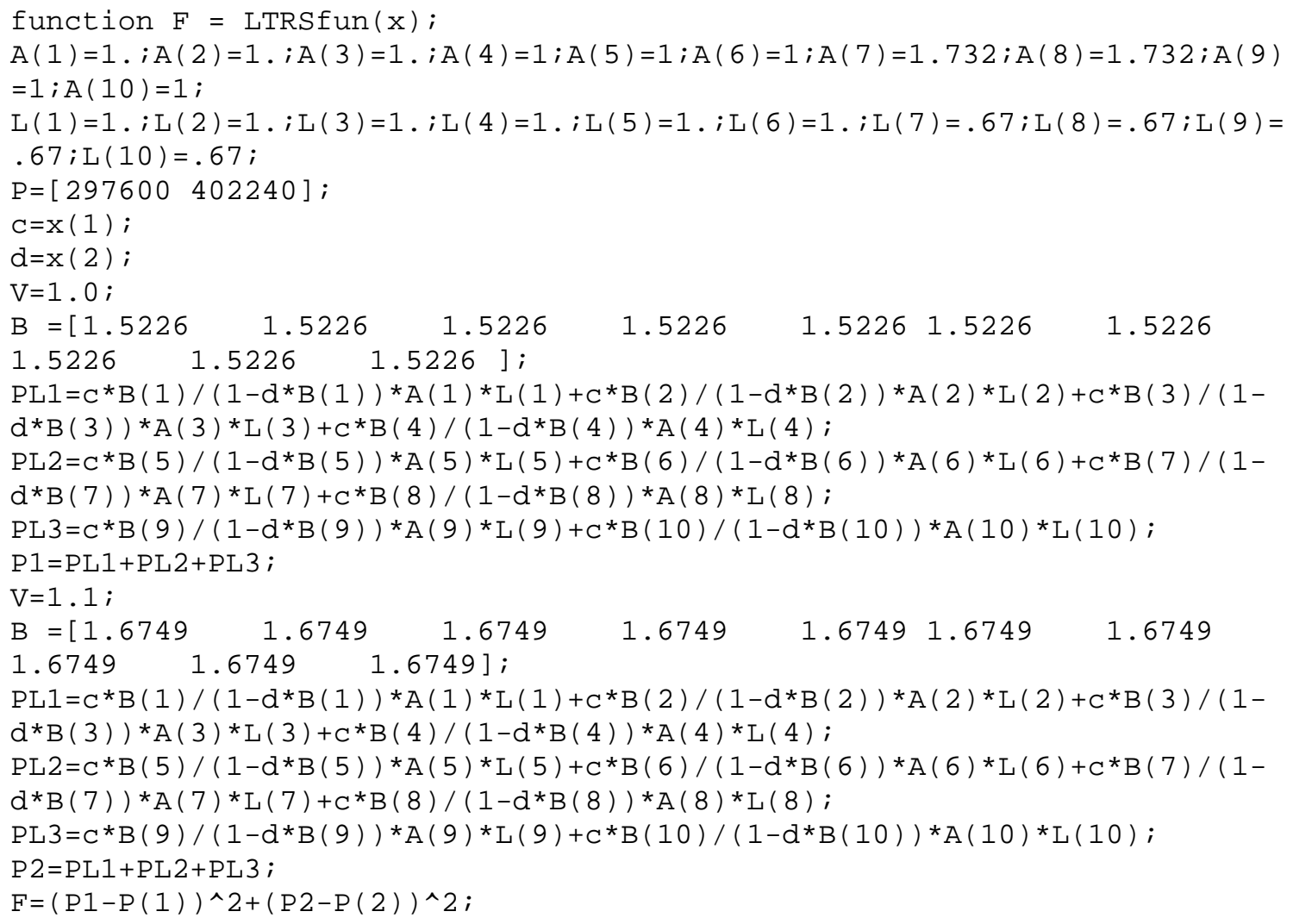




\section{Appendix B.19: AHS.m}

Parameter estimation of Hysteresis Loss curve for Shell-form transformer

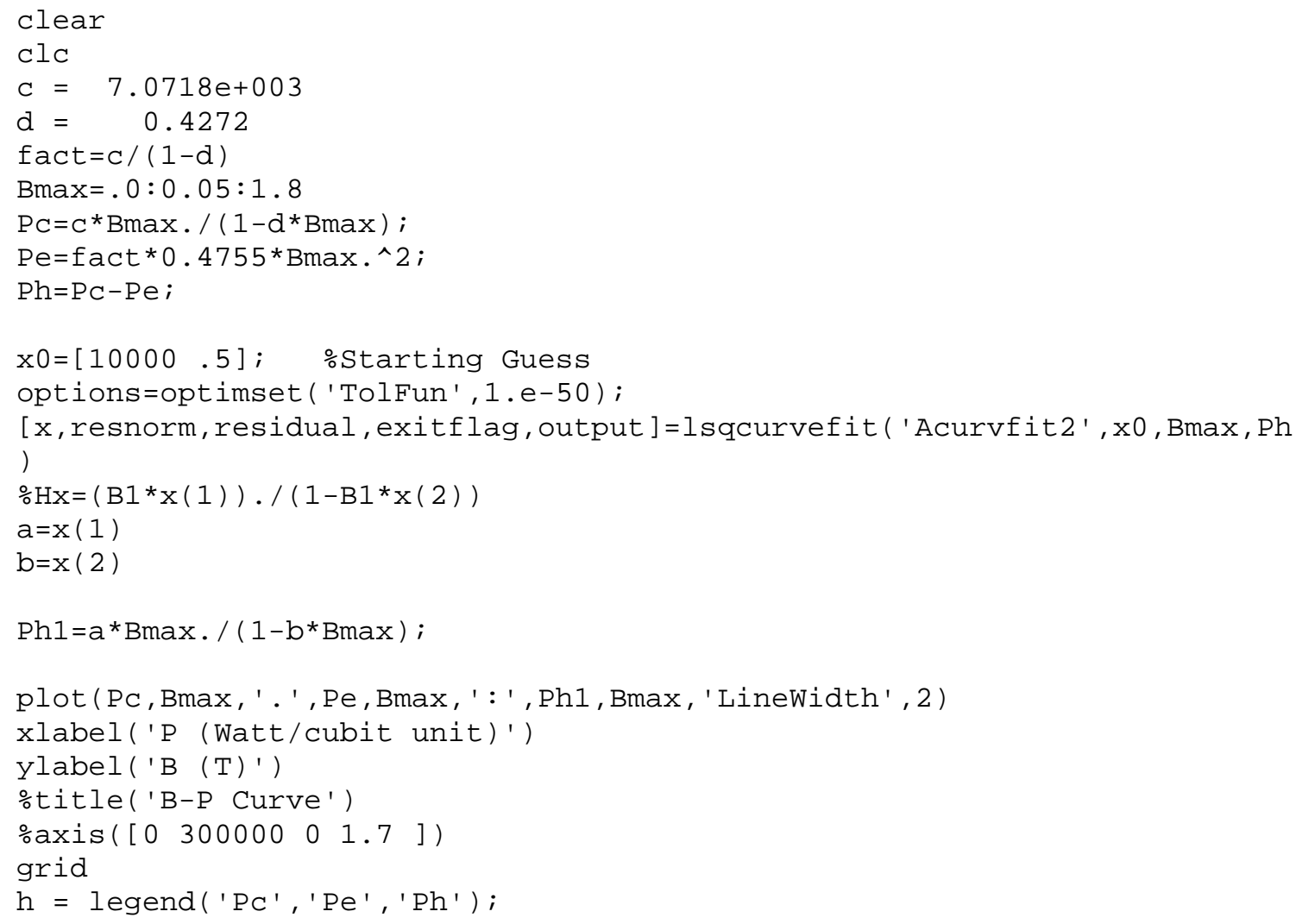

\section{Appendix B.20: Acurvfit2.m}

Parameter estimation of hysteresis loss curve for shell-form transformer

function $F=\operatorname{curvfit} 2(\mathrm{x}, \mathrm{B})$;

$\mathrm{F}=(\mathrm{B} * \mathrm{X}(1)) . /\left(1-\mathrm{B}^{*} \mathrm{X}(2)\right)$;

\section{Appendix B.21: AhsA.m}

Estimation of maximum coercive force for shell-form transformer

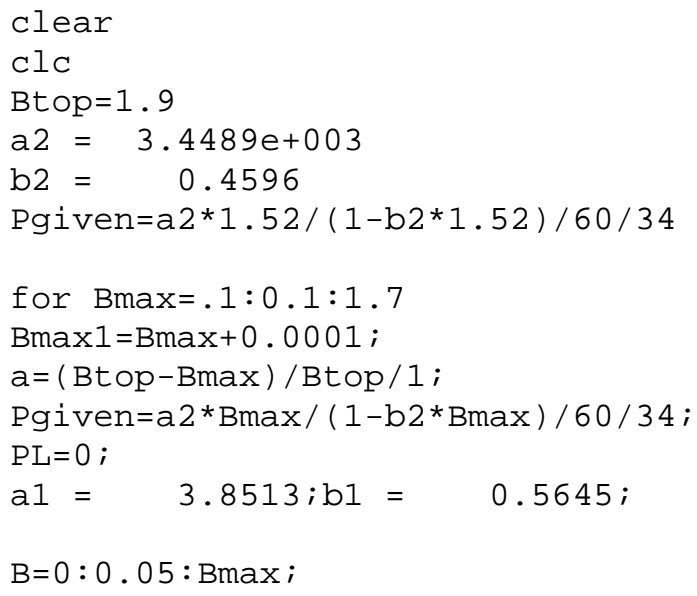




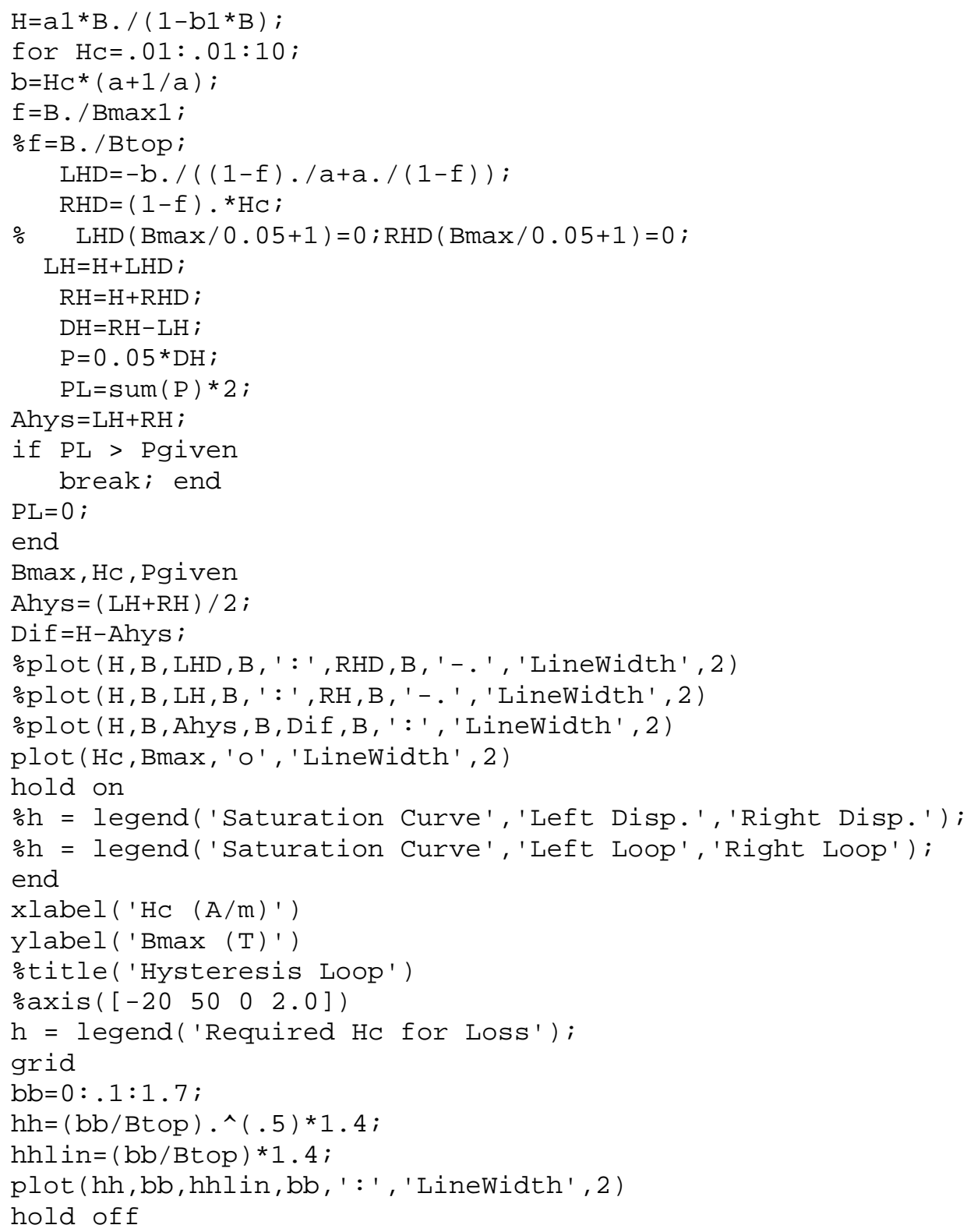




\section{APPENDIX C: TRANSFORMER FACTORY TEST REPORT}

\section{TRANSFORMER TEST REPORT}

Date of Test $6 / 3 / 71$

Customer's Order C-67899

Our Order $\quad$ C-OL $4070-5$

TypeOA/FOA/FOA Phase 3 Cycles 60 Ris $55^{\circ} / 65^{\circ} \mathrm{CTaps}$ See N.P. Dwg. 1307256 Spec. 13018

H. V. Volts 345000 Grd.X/199200 L. V. Volts 118000 Grd. I/68200 T.V. Volts 138001

KVA $296000 / 3914000 / 420000 *$ KVA $296000 / 294000 / 490000 *$

KVA $77000 / 102657 / 128333 *$

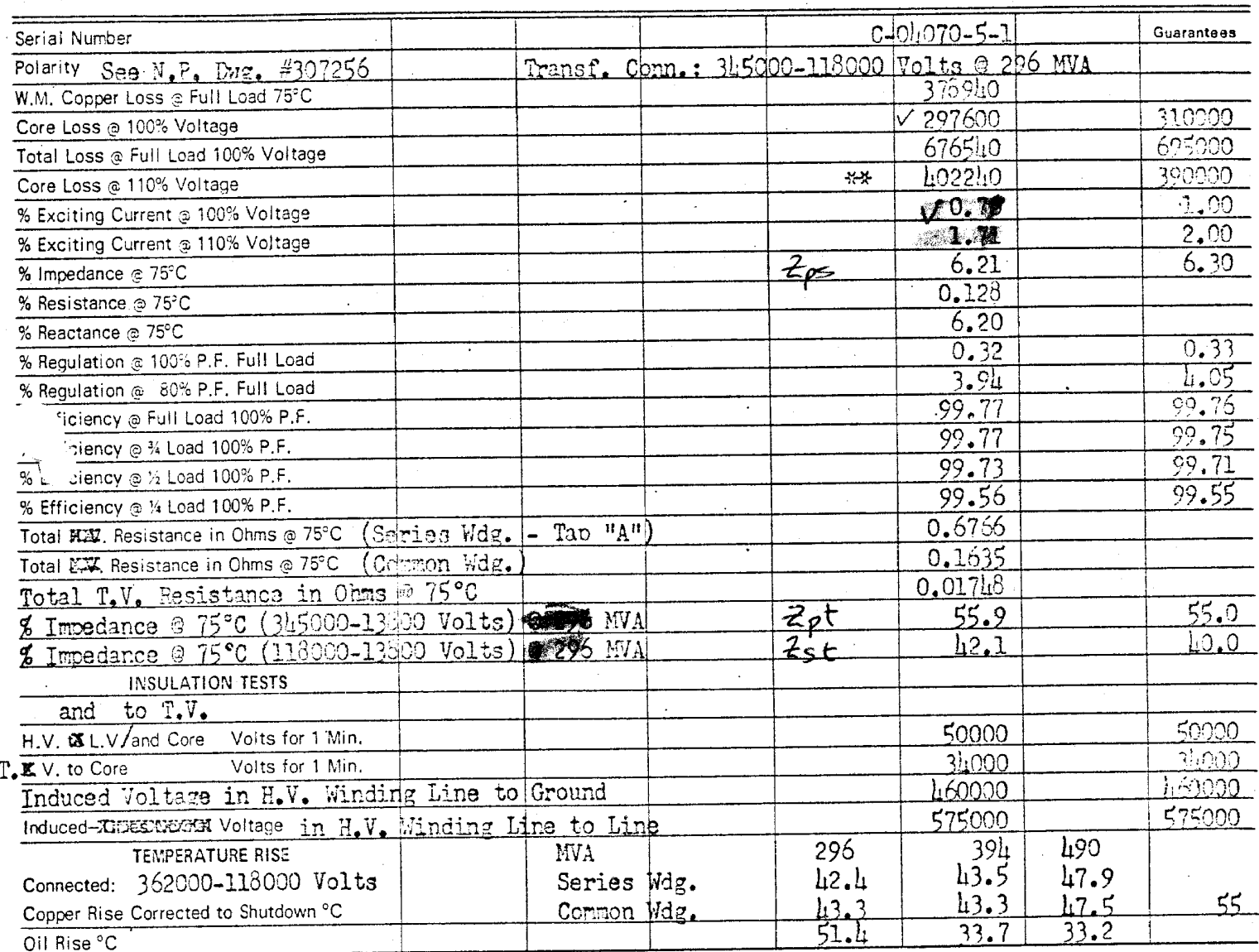

Oil Rise ${ }^{\circ} \mathrm{C}$

Unless otherwise specified the above Tests are in accordance with the latest A. S. A. and N. E. M. A. Standards.

Remarks: (3) $77000 \mathrm{KVA}$ Q 102667 NIA

T.V. Gradient ${ }^{\circ} \mathrm{C}:$ KVA $65^{\circ} \mathrm{C}$ Rise: H.V. and L.V. 330000/440000/550000: T.T. $-86240 / 114987 / 143733$. 128333 57A

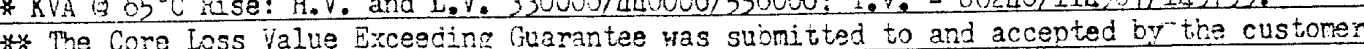

is transfomer satiafactorily withstood Imoulse fests. See Imulse fest Peoprt.

transfomar satisfactorily ithstood Switching Surge Tests. See Switching Surse Teat Renor Se Jage $\#$ for additional test periormance data. 